\title{
Regulation of Drosophila melanogaster body fat storage by store-operated calcium entry
}

\author{
Dissertation \\ for the award of the degree \\ "Doctor rerum naturalium" \\ of the Georg-August-Universität Göttingen
}

within the doctoral degree program (Genes and Development)

of the Georg-August University School of Science (GAUSS)

Submitted by

Yanjun $\mathrm{Xu}$

From Kaifeng

Göttingen, 2017 


\section{Thesis advisory committee}

Prof. Dr. Ronald P. Kühnlein

Institute of Molecular Biosciences, University of Graz \&

Research group of Molecular Physiology

Max Planck Institute for Biophysical Chemistry

Prof. Dr. Ahmed Mansouri

Research group of Molecular Cell Differentiation,

Max Planck Institute for Biophysical Chemistry \&

Department of Clinical Neurophysiology

University Medical Center Göttingen, Georg-August University, Göttingen

Prof. Dr. Jörg Großhans

Institute of Developmental Biochemistry

University Medical Center Göttingen, Georg-August University Göttingen

\section{Members of the examination board:}

Referee: Prof. Dr. Ronald P. Kühnlein Institute of Molecular Biosciences, University of Graz \&

Research group of Molecular Physiology

Max Planck Institute for Biophysical Chemistry

Co-referee: Prof. Dr. Ahmed Mansouri

Research group of Molecular Cell Differentiation, Max Planck Institute for Biophysical Chemistry \&

Department of Clinical Neurophysiology

University Medical Center Göttingen

Georg-August University Göttingen

\section{Other members of the examination board:}

Prof. Dr. Jörg Großhans

Institute of Developmental Biochemistry

University Medical Center Göttingen, Georg-August University Göttingen

Prof. Dr. André Fiala

Department of Molecular Neurobiology of Behavior

Johann-Friedrich-Blumenbach-Institute for Zoology and Anthropology,

Georg-August University Göttingen

Prof. Dr. Ernst Wimmer

Department of Developmental Biology

Johann-Friedrich-Blumenbach-Institute for Zoology and Anthropology,

Georg-August University Göttingen

Dr. Nico Posnien

Emmy-Noether Group

Department of Developmental Biology

Johann-Friedrich-Blumenbach-Institute for Zoology and Anthropology,

Georg-August University Göttingen

Date of oral examination: April 28, 2017 


\section{Pre-release parts of the dissertation}

\section{Publication}

1. Baumbach, J., Xu, Y*., Hehlert, P., and Kühnlein, R.P. (2014b). Gaq, Gy1 and Plc21C control Drosophila body fat storage. J. Genet. Genomics 41, 283-292. doi:10.1016/j.jgg.2014.03.005.

*Indicates equal contribution. This publication was collaborated with Dr. J. Baumbach, Dr. P. Hehlert, and Dr. R. P. Kühnlein. The Figure 3, and Figure 4A in the thesis were collaborated with Dr. J. Baumbach and based on the publication Fig. 1D, Fig. 2A. The Figure 4B in the thesis was kindly provided by Dr. J. Baumbach and based on the publication Fig. $2 \mathrm{~B}$.

2. Gáliková, M., Diesner, M., Klepsatel, P., Hehlert, P., Xu, Y., Bickmeyer, I., Predel, R., and Kühnlein, R.P. (2015). Energy homeostasis control in Drosophila adipokinetic hormone mutants. Genetics 201, 665-683. doi: 10.1534/genetics.115.178897.

Startle-induced climbing assay and its material \& method part were carried out and wrote by Yanjun $\mathrm{Xu}$. The material \& method writing of startle-induced climbing assay in the PhD thesis was based on this publication.

3. Gáliková, M., Klepsatel, P., Xu, Y., and Kühnlein, R.P. (2017). The obesity-related adipokinetic hormone controls feeding and expression of neuropeptide regulators of Drosophila metabolism. Eur. J. Lipid Sci. Technol. 119. 1600138. doi:10.1002/ejlt.201600138.

qPCR primers (they target following gene respectively: CCHa2, $\mathrm{Tk}, \mathrm{Crz}, \mathrm{ImpL} 2$ ) were designed by Yanjun $\mathrm{Xu}$, which were also used in the $\mathrm{PhD}$ thesis. 


\section{Table of contents}

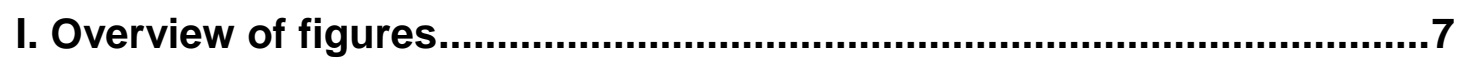

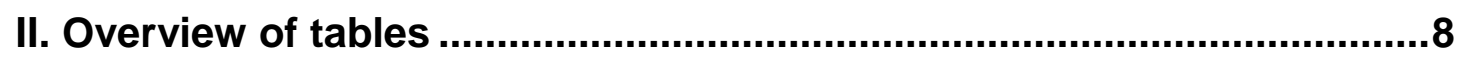

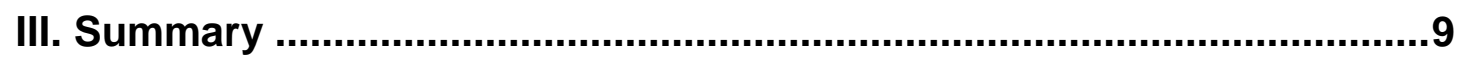

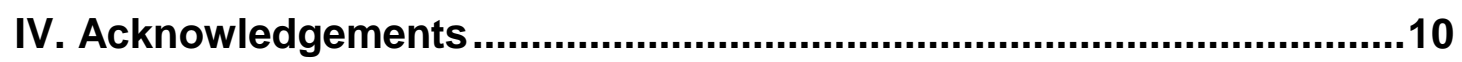

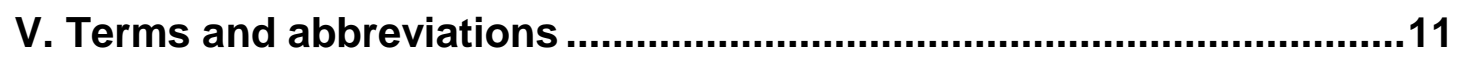

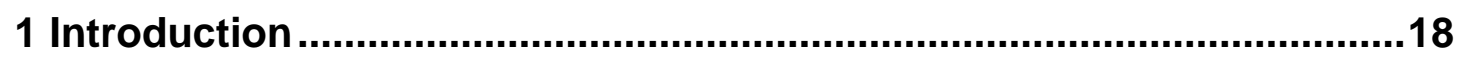

1.1 The contributors of obesity ....................................................................19

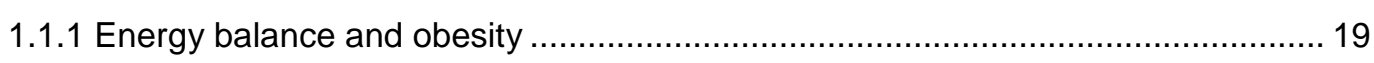

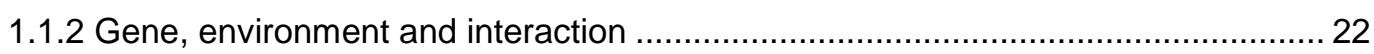

1.2 Drosophila melanogaster as an emerging model for obesity research .....24

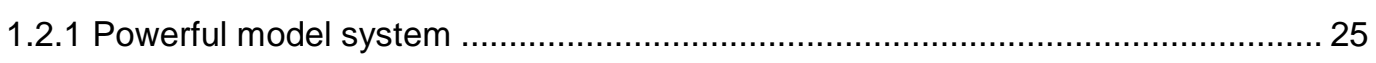

1.2.2 Energy metabolic organ systems in Drosophila and human ................................ 29

1.2.3 Energy reserve storage in Drosophila and human .......................................... 30

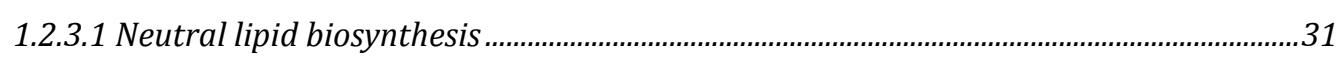

1.2.3.2 Lipid droplet (LD) biogenesis.............................................................................................33

1.2.3.3 LD formation, growth and expansion …………….........................................................33

1.2.4 Mobilization of the energy storage in Drosophila and human ............................... 34

1.2.5 Inter-organ regulation of energy homeostasis in Drosophila and human .............. 35

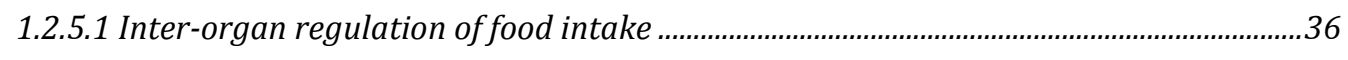

1.2.5.2 Inter-organ regulation of energy storage ............................................................................

1.2.5.3 Inter-organ regulation of energy mobilization......................................................................38

1.2.6 Cellular regulation of energy homeostasis in Drosophila and human ................... 39

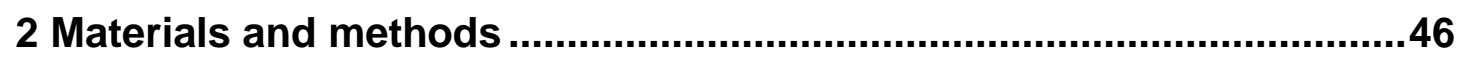

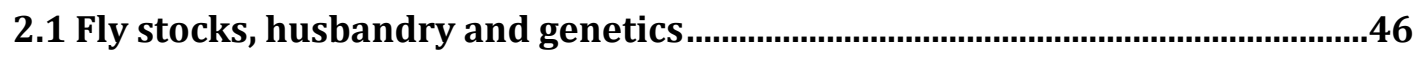

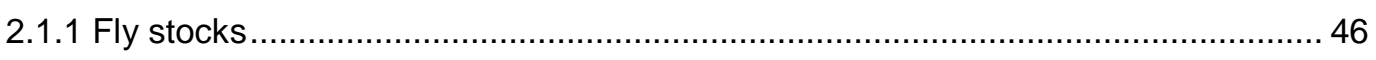

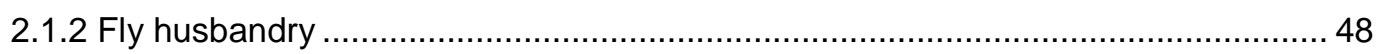

2.1.3 Fly genetics for non-conditional RNAi expression and CaLexA expression .......... 49 
2.1.4 Fly genetics for temperature-shift induced RNAi expression .............................. 49

2.1.5 Fly genetics for transient drug-feeding induced RNAi expression ....................... 50

2.1.6 Stim cDNA or RNAi resistant cDNA construct transformation ............................ 50

2.1.7 Genetic crosses for combining transgenes or mutations together ....................... 51

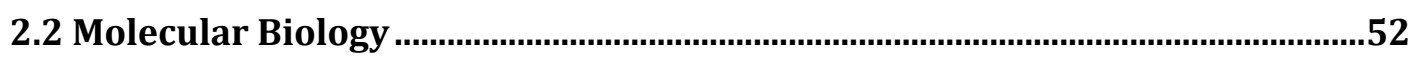

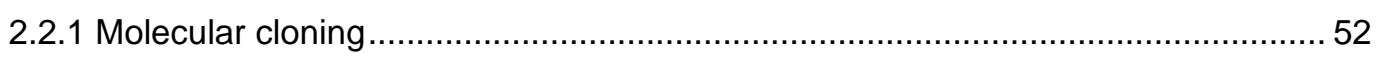

2.2.1.1 Preparation of chemical competent bacteria and their transformation ..........................52

2.2.1.2 Colony picking, E.coli culture and plasmid isolation ..........................................................53

2.2.1.3 DNA restriction analysis and ligations...............................................................................53

2.2.1.4 Vector construction of pUASTattB-Stim RNAi1 resistant cDNA RA.................................53

2.2.2 Single fly genotyping by polymerase chain reaction (PCR) ............................... 55

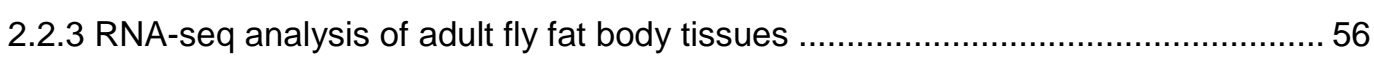

2.2.4 Reverse Transcription and quantitative PCR (RT-qPCR) ................................. 58

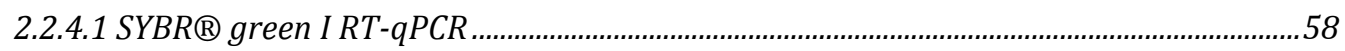

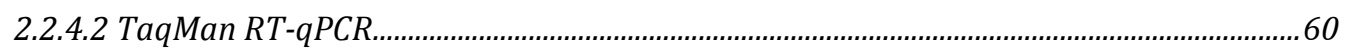

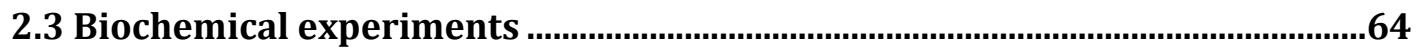

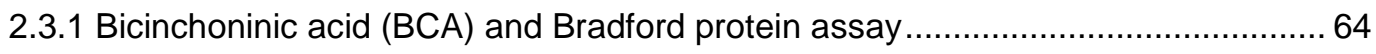

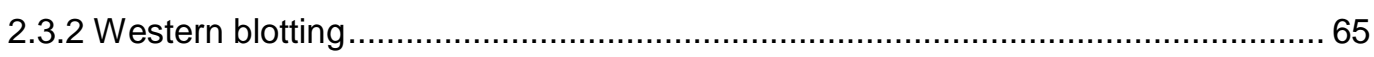

2.3.3 Coupled colorimetric assay (CCA) for TAG equivalents quantification ..................66

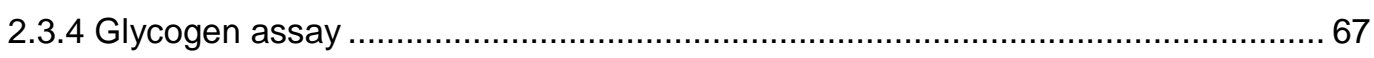

2.3.5 Thin layer chromatography (TLC) for neutral lipids determination ........................6. 67

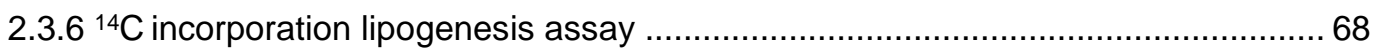

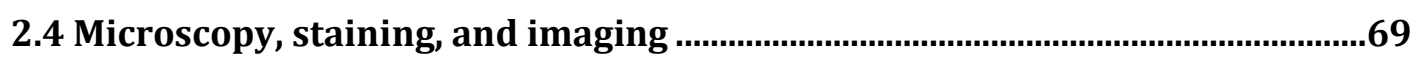

2.4.1 Dissection, microscopy and imaging of adult flies .............................................. 69

2.4.2 Ex vivo staining of adult fat body cells and confocal imaging ............................. 70

2.4.2.1 Ex vivo staining of dissected fat body tissues .........................................................................70

2.4.2.2 Confocal imaging of ex vivo stained dissected fat body tissues ........................................70

2.4.2.3 Fat body cell and lipid droplet size quantification ..............................................................71

2.4.3 Confocal imaging of fat body expressing CaLexA GFP ....................................... 72

2.4.4 5-ethynyl-2'-deoxyuridine (EdU) staining of adult fly fat body cell and midgut

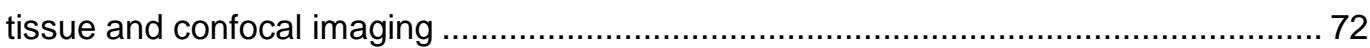

2.4.5 Akh and dILP-2 immunostaining and confocal imaging .................................... 73

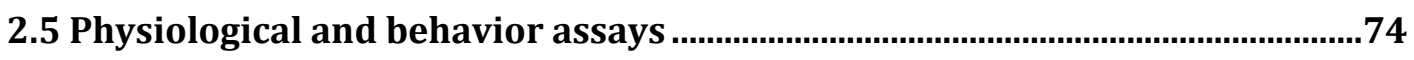

2.5.1 Wet body weight measurement ................................................................. 74

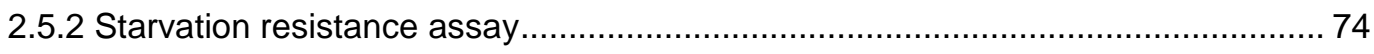

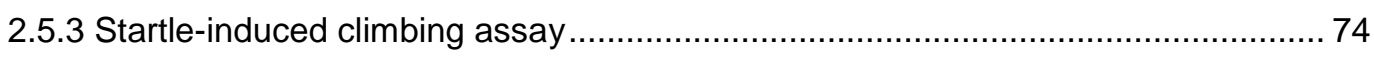

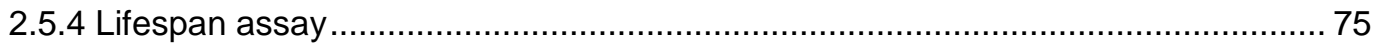

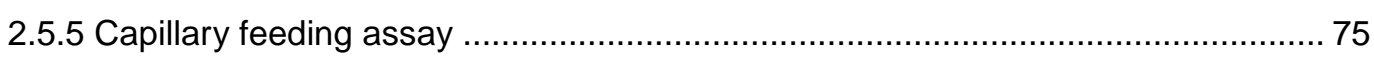

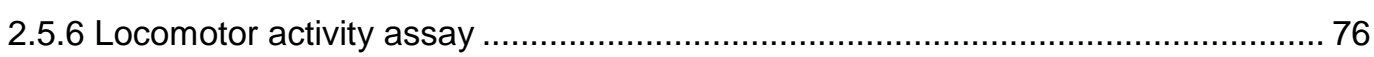

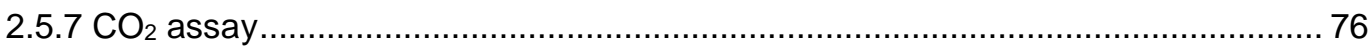


3.1 Identification of candidate genes which act upstream of Stim and regulate body fat storage

3.1.1 Long-term and individual modulation of Akh, AkhR, Gy1, Gaq49B, PIc21C, and Stim gene expression levels affects the fat storage of adult flies.

3.1.2 Long-term and individual down-regulation of Akh, AkhR, Gy1, Gaq49B, Plc21C, and Stim expression levels results in a decrease of cytoplasmic calcium levels of the fat body cells.

\subsection{Identification and characterization of Long-term Impairment of STIM} function induced Obesity (LISO) in adult flies.

3.2.1 Stim-Transient temperature-shift Induced RNAi expression (Stim-TtI) in adult Drosophila males cause a long-term functional impairment of STIM and promotes longterm fat storage increase.

3.2.2 Stim-Transient drug-feeding Induced Stim RNAi expression (Stim-Tdl) in adult Drosophila males causes a long-term functional impairment of STIM and promotes longterm fat storage increase.

3.2.3 Stim-Ttl causes a long-term functional impairment of STIM and promotes long-term fat storage increase in adult flies except in mated females

3.2.4 Ttl of candidate gene AkhR which acts upstream of Stim promotes also an

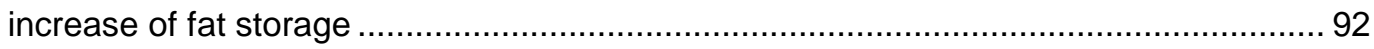

3.2.5 Long term Impairment of STIM function induced Obesity (LISO) ....................... 93

3.2.6 LISO affects the physiological performances of flies................................ 97

\subsection{Dysfunctional energy homeostasis in response to a Long-term Impairment}

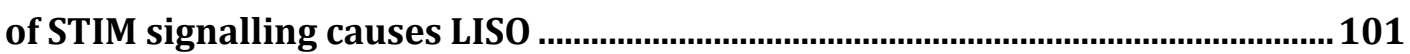

3.3.1 Dysfunctional energy homeostasis after Stim-TtI contributes to t-LISO............... 101

3.3.2 Dysfunctional energy homeostasis after Stim-Tdl contributes to d-LISO............ 104

3.4 Molecular, cellular and system investigations on mechanisms underlying the

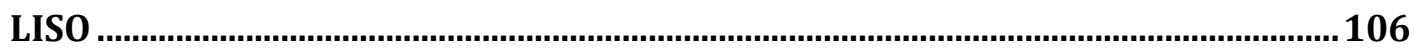

3.4.1 Mechanism of long-term reduction of gene mRNA expression after Ttl 107

3.4.2 RNA-seq analysis reveals candidate regulator genes in fat body tissue during LISO

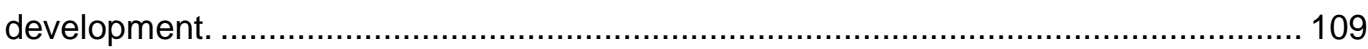

3.4.3 Akh signalling contributes to LISO via increased food intake ............................. 112

3.4.4 Selective insulin signalling resistance occurs in LISO flies ........................... 116

3.4.5 The expression of lipolysis and $\beta$-oxidation genes is reduced in LISO flies........ 121

3.4.6 The expression of malate/aspartate shuttling genes is reduced in LISO flies ..... 124

4 Discussion.

4.1 Akh/AkhR signalling regulates fat storage via G $\gamma 1$-Goq49B-PIc21C-Stim modulated $\mathrm{Ca}^{2+}$ level changes. 
4.2.1 Transient Stim RNAi expression in fat body promotes long-term fat storage increase

4.2.2 LISO is independent of gender and age except in mating female flies 135

4.2.3 $\mathrm{Ttl}$ of putative SOCE upstream genes promotes long-term fat storage increase 136

4.2.4 LISO provides an attractive model for obesity and related diseases research .... 138

4.3 Dysfunctional energy homeostasis causes LISO

4.4 Molecular and cellular mechanisms underlying LISO

4.4.1 Stim-Transient Induced RNAi expression (Stim-TI) mediates long-term impairment of STIM function possibly via long-lasting small interfering RNA

4.4.2 Long-term impairment of STIM function is accompanied by alterations of gene expression in multiple pathways

4.4.3 Akh signalling contributes to LISO by promoting the food intake.

4.4.4 Long-term impairment of STIM function in fat storage tissue contribute to selective insulin resistance and lipid synthesis in LISO flies

4.4.5 Stim-Ttl reduces the expression of genes involved in lipolysis and mitochondrial functions

4.5 Fat body tissue is a key player in the inter-organ regulatory network to maintain energy homeostasis

\section{Promovierenden-Erklärung der Georg-August-Universität Göttingen}




\section{Overview of figures}

Figure 1. The organ systems of Drosophila melanogaster are functionally analogous to those in human. 29

Figure 2. Scheme of fat storage control by the Store-Operated Calcium Entry (SOCE) in response to GPCR/Gaq/Plc pathway, as well as by Akh/AkhR/Gas/adenylyl cyclase/cAMP, and insulin signalling pathways 41

Figure 3. Long-term and individual modulation of Akh, AkhR, Gy1, Gaq49B, PIc21C and Stim expression affects cellular fat storage.

Figure 4. Long-term and individual down-regulation of AkhR, Gy1, Gaq49B, Plc21C, and Stim expression reduces cytoplasmic calcium levels of fat body cells 82

Figure 5. Stim-Transient temperature-shift induced RNAi expression (Ttl) in fat storage tissues of Drosophila adult males causes a long-term impairment of STIM function and promotes long-term body fat storage increase. 85

Figure 6. Stim-Transient drug-feeding induced RNAi expression (Tdl) in Drosophila adult males causes a long-term impairment of STIM function and promotes a long-term fat storage increase. 89

Figure 7. Stim-Ttl promotes long-term fat storage increase in adult flies except in mated females.

Figure 8. Ttl of Stim upstream candidate gene- $A k h R$ promotes long-term fat storage increase 93

Figure 9. Stim-Ttl in fat storage tissue of Drosophila adult males causes long-term Impairment of STIM function induced Obesity (LISO). 95

Figure 10. LISO affects the physiological performances of flies 98

Figure 11. Increased food intake and less energy expenditure after Stim-TtI contribute to t-LISO 102

Figure 12. Increased food intake, increased lipid biosynthesis and less energy expenditure after Stim-Tdl contribute to d-LISO 105

Figure 13. Long-term existence of siRNAs from AkhR-Ttl and their effects on body fat storage and $A k h R$ mRNA expression 107

Figure 14. RNA-seq analysis identifies gene expression changes in LISO fly fat body tissue 110

Figure 15. Akh signalling contributes to LISO in response to Stim-TtI by promoting the food intake. 114

Figure 16. Insulin signalling resistance occurs in peripheral tissues of LISO adult males 
Figure 17. mRNA expressions of the genes involved in lipolysis and $\beta$-oxidation were reduced in LISO fly fat body tissue

Figure 18. mRNA expressions of the genes encoding enzymes participating in malate-aspartate shuttle between cytoplasm and mitochondria were reduced in LISO fly fat body tissue 125

Figure 19. (Preliminary data) Co-expression of Stim RNAi and RNAi resistant cDNA does not rescue the long-term body fat storage increase 132

Figure 20. (Preliminary data) Body fat content changes of flies with Stim-Tdl in different organ tissues of adult male flies, or in dllp- 6 candidate mutant 134

Figure 21. (Preliminary data) Ttl of Stim upstream candidate gene promotes longterm fat storage increase. 137

Figure 22. Scheme representing a model of how energy regulatory network is disturbed in the fat body cell and at inter-organ level in LISO flies. 155

Figure 23. (Supplementary data) Stim genomic localization, Stim RNAi target region and locomotor activity counts per 5 min of adult male flies over the 11 days after Stim-TtI 157

Figure 24. (Supplementary data) Sample variance analysis of RNA-seq data, and Gene Ontology analysis (biological process) of up and down-regulated genes 158

Figure 25. (Supplementary data) Gene expression level changes of food intakerelated neuropeptide and neurotransmitter signaling genes in the heads of adult male flies after Stim-Ttl 160

Figure 26. (Supplementary data) Selective insulin resistance occurs in peripheral tissues of LISO adult males. 161

\section{Overview of tables}

Table 1 Information of fly stocks 46

Table 2 Information of plasmids 54

Table 3 PCR primers for sequencing or genotyping 55

Table 4 RT-qPCR primers 62 


\section{Summary}

The worldwide prevalence of obesity and related chronic diseases is a major health problem. Obesity is driven by energy imbalance in obesogenic environments, diets and lifestyles. The underlying mechanisms of energy imbalance involve complex interactions among multilayered regulatory networks in biological system, most components of which are conserved from fruit fly to human. Recently, calcium signaling has been identified as a central regulator of energy homeostasis, which integrates the cellular metabolic information in a variety of organs including adipose tissue, liver, and the brain. A link between acute modulation of calcium signaling in fat storage tissue and energy homeostasis has been investigated in previous research by interfering with the activity of a key calcium signaling component encoded by the gene Stromal interaction molecule (Stim), which regulates the storeoperated calcium entry (SOCE). But how chronically impairing calcium signaling in fat storage tissue drives obesity remained to be elucidated. Here I present experimental evidence that $G$ proteins $G \gamma 1$, Gaq49B, and phospholipase C at least partially mediate the activity of the adipokinetic hormone Akh/ adipokinetic hormone receptor (AkhR) signaling (functionally similar to mammalian glucagon), which mobilize the fat storage via the Stim-mediated SOCE. Using molecular genetics, transcriptome analysis, imaging, biochemical, as well as physiological and behavioral methods, I found that Stim-Transiently Induced (TI) RNAi transgene expression in the adult fat storage tissue of the fly causes a long-term impairment of STIM function and accumulation of body fat. This Long-term Impairment of STIM function induced Obesity (LISO) is primarily caused by an increased food intake of the flies, aggravated by reduced metabolic rate and locomotor activity. At the molecular level, Stim-TI in fat storage tissue of the fly caused increased lipid biosynthesis, reduced the expression of genes involved in lipolysis and fatty acid $\beta$-oxidation. At the cellular and organ level, Stim-TI induced hyperphagia via an increased secretion of Akh from the neuroendocrine corpora cardiaca cells. The results also suggest that LISO flies develop Akh and insulin signaling resistance specifically in Drosophila adipose tissue. Taken together, the long-term impairment with Stim function that results from Stim-TI has dramatic consequences for the regulatory network that controls energy homeostasis. LISO provides a powerful and straightforward model for the study on the complex interactions of regulatory networks underlying obesity and its related diseases. 


\section{Acknowledgements}

This PhD work was conducted in Research Group of Molecular Physiology, and Department of Molecular Developmental Biology, at Max Planck Institute for Biophysical Chemistry (MPlbpc), Göttingen, Germany. This PhD work was carried out under the supervision from my supervisor Prof. Dr. Ronald Kühnlein. Besides, this PhD work was in part financially supported by the German Research Foundation (Die Deutsche Forschungsgemeinschaft) and the Max Planck Society (Die MaxPlanck-Gesellschaft). I thank Prof. Dr. Ronald Kühnlein for offering me such a great chance to do my PhD study at MPIbpc. I thank Prof. Dr. Herbert Jäckle for the wonderful fruit fly and biology research facility, Coffee, last half-year financial support, and considerate advice.

Especially, I would like to thank Prof. Dr. Ronald Kühnlein. His continuous supports enabled me to finish the dissertation. Numerous discussion, emails, and comments guided and inspired me to do critical thinking, and to carry out the project in a better way. I am very grateful to other two members of my thesis advisory committee: Prof. Dr. Ahmed Mansouri, and Prof. Dr. Jörg Großhans. Thanks for their joining my thesis committee meetings, sharing their insights, and offering me their critical advice.

I thank my colleagues: Dr. Jens Baumbach, Dr. Philip Hehlert, Dr. Martina Gáliková, Dr. Peter Klepsatel, Dr. Yuanbin Xie etc. Without their help, it would have been more difficult to finish the PhD work. I thank the bachelor student Annika Franziska Borcherding, who helped me to do the important part of the project. I also thank technician (Regina Krügener, Iris Bickmeyer), and student assistants (Jana Laura Heidemann, Vanessa Kernke, Pilar Mata Tutor, Mike Pieczarek) for their excellent technical assistance. I am grateful to colleagues in the department for great fly food, and experiment assistance: Ulrike Borchhardt, Karin Hartwig, Claudia Koch, Alexey Matyash, and Ralf Pflanz etc.

I would like to thank Göttingen Graduate School for Neurosciences, Biophysics, and Molecular Biosciences (GGNB) for enrolment, nice coordination, and different courses. I thank fly stock centers (BDSC, VDRC), and fruit fly research community for offering, and sharing research materials. I also thank my Chinese friends in Göttingen for their manifold help. Finally, I would like to say many thanks to my parents, my sisters for their understanding and unparalleled support. 


\begin{tabular}{|c|c|}
\hline$A C$ & adenylate cyclase \\
\hline ACADVL & Acyl-CoA Dehydrogenase, Very Long-Chain abbreviatec \\
\hline ACS & acyl-CoA synthetase \\
\hline ACSL & acyl-CoA synthetase long chain \\
\hline ACC & Acetyl-CoA carboxylase \\
\hline Act5C & Actin 5C \\
\hline AGPAT & 1-acyl-sn-glycerol-3-phosphate O-acyltransferases \\
\hline Akh & Adipokinetic hormone \\
\hline AkhR & Adipokinetic hormone receptor \\
\hline AMPK & AMP-activated kinase \\
\hline ATP & adenosine triphosphate \\
\hline ATP $\operatorname{syn} \beta$ & ATP synthase, $\beta$ subunit \\
\hline ATGL & adipose triglyceride lipase \\
\hline attB & 34 bp minimal DNA sequence recognized by PhiC31 \\
\hline attP & 39 bp minimal DNA sequence recognized by PhiC31 \\
\hline $\mathrm{BCA}$ & Bicinchoninic acid \\
\hline BDSC & Bloomington Drosophila Stock Center \\
\hline blw & bellwether \\
\hline BMI & Body Mass Index \\
\hline$b m m$ & brummer \\
\hline BOSS & Bride of sevenless \\
\hline bp & base pair \\
\hline BP & biological process \\
\hline BSA & Bovine serum albumin \\
\hline $\mathrm{iCa}^{2+}$ & intracellular calcium ion level \\
\hline CaLexA & calcium-dependent nuclear import of Lex A \\
\hline CanA-14F & Calcineurin $\mathrm{A}$ at $14 \mathrm{~F}$ \\
\hline CAS9 & CRISPR associated protein 9 \\
\hline CAMP & Cyclic adenosine monophosphate \\
\hline cDNA & complementary deoxyribonucleic acid \\
\hline $\mathrm{cc}$ & corpora cardiaca \\
\hline $\mathrm{CC}$ & Cellular Component \\
\hline CCA & Coupled colorimetric assay \\
\hline $\mathrm{CCHa} 2$ & CCHamide 2 \\
\hline CCK & cholecystokinin \\
\hline
\end{tabular}


CCT

C. elegans

CLAHE

CoA

$\mathrm{CO} 2$

CPT

CPT1

CRAC

CrebB

CRISPR

$\mathrm{CRH}$

$\mathrm{C}_{\mathrm{T}}$

CTLN2

Crz

DAG

daGS

DAPI

daw

DGAT

$d b$

Dh

DMSO

DNA

dsRNA

DSK

DTT

DVD

EDTA

EdU

EGTA

e.g.

Egr

EMS

ER

etc

ETC

4EBP
CTP:phosphocholine cytidylyltransferase

Caenorhabditis elegans

Contrast Limited Adaptive Histogram Equalization

coenzyme A

carbon dioxide

CDP choline:diacylglycerol choline-phosphotransferase

carnitine palmitoyltransferase 1

$\mathrm{Ca}^{2+}$ release-activated $\mathrm{Ca}^{2+}$ channels

Cyclic-AMP response element binding protein $B$

clustered regularly interspaced short parlindromic repeats

corticotropin-releasing hormone

Cycle Threshold

adult-onset type II citrullinemia

Corazonin

Diacylglyceride

daughterless GeneSwitch

4',6-diamidino-2-phenylindole

dawdle

acyl-CoA:diacylglycerol acyltransferases

Diabetes mice mutation

Diuretic hormone

Dimethy sulfoxide

deoxyribonucleic acid

double-stranded RNA

Drosulfakinin

Dithiothreitol

Digital Versatile Disc

Ethylenediaminetetraacetic acid

5-ethynyl-2'-deoxyuridine

Ethylene glycol-bis( $\beta$-aminoethyl ether)-N,N,N',N'-tetraacetic acid to list examples

Eiger

Ethyl methanesulfonate

Endoplasmatic Reticulum

et cetera means and so on

Electron Transport Chain

eukaryotic translation initiation factor $4 \mathrm{E}$ binding protein 


$\begin{array}{ll}\text { FA } & \text { fatty acids } \\ \text { FADH2 } & \text { flavin adenine dinucleotide } \\ \text { FAS } & \text { fatty acid synthetase } \\ \text { FB } & \text { fat body } \\ \text { FITM/FIT } & \text { fat storage-inducing transmembrane protein } \\ \text { FOXO } & \text { forkhead box, sub-group O } \\ \text { FTO } & \text { Fat mass and Obesity associated } \\ \text { 5-HT7 } & \text { 5-hydroxytryptamine (serotonin) receptor 7 } \\ \text { g } & \text { Gram } \\ \text { Gal4 } & \text { A Yeast transcription activator protein factor } \\ \text { GBP } & \text { growth blocking peptide } \\ \text { GCK } & \text { Glucokinase } \\ \text { GDP } & \text { Gross domestic product } \\ \text { gDNA } & \text { genomic DNA } \\ \text { GNP } & \text { Gross national product } \\ \text { GO term } & \text { Gene Ontology term } \\ \text { GO kit } & \text { Glucose assay with Glucose oxidase } \\ \text { Gö-food } & \text { Göttingen food } \\ \text { Got } & \text { Glutamate oxaloacetate transaminase } \\ \text { GPAT } & \text { glycerol phosphate acyltransferase } \\ \text { GPCR } & \text { G protein-coupled receptor } \\ \text { GS } & \text { GeneSwitch } \\ \text { GWAS } & \text { Genome-wide association studies } \\ \text { H } & \text { insulin-like receptor } \\ \text { h } & \text { insulin producing cells } \\ \text { Hex-C } & \text { insulin-like peptide } \\ \text { HiLo } & \text { hour } \\ \text { Hnf4 } & \text { Hexokinase C } \\ \text { HRP } & \text { (http://imagej.net/Visualization) } \\ \text { Hsl } & \text { Hepatocyte nuclear factor 4 } \\ \text { i.e. } & \text { Horseradish peroxidase } \\ \text { IGBP7 } & \text { That is to say, in other words } \\ \text { ILP } & \text { impL2 }\end{array}$




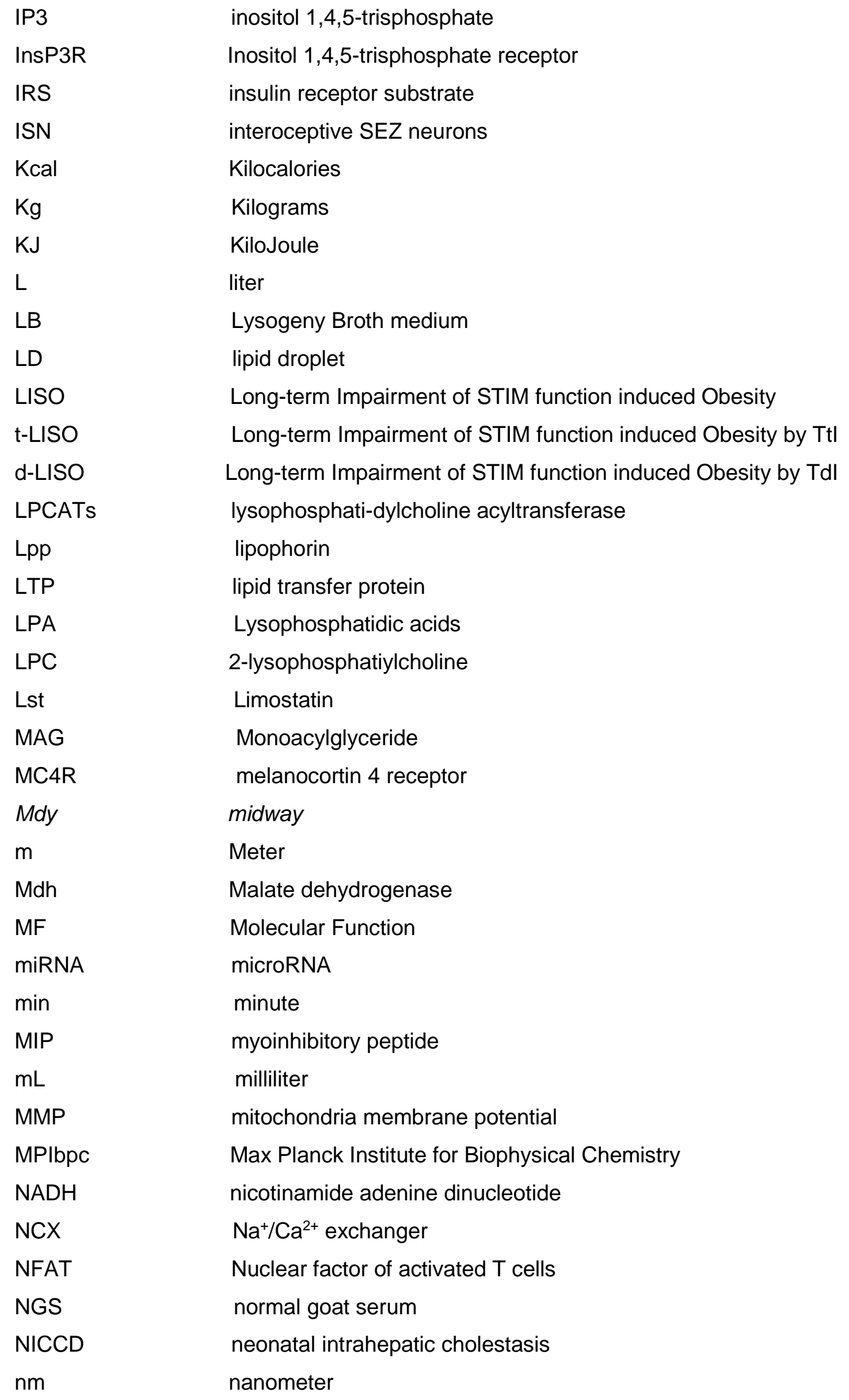




\begin{tabular}{|c|c|}
\hline NPY & neuropeptide $Y$ \\
\hline NPF & neuropeptide F \\
\hline$a b$ & Obesity mice mutation \\
\hline OD & Optical density \\
\hline PA & Phosphatidic acid \\
\hline PAGE & Polyacrylamide gel electrophoresis \\
\hline PAP1 & $\mathrm{Mg}^{2+}$-dependent PA phosphatases \\
\hline PBS & Phosphate-buffered saline \\
\hline PBST & Triton ${ }^{\circledR} \mathrm{X}-100$ diluted in PBS \\
\hline PC & Phosphatidylcholine \\
\hline PCA & principal component analysis \\
\hline PCR & polymerase chain reaction \\
\hline PE & Phosphatidylethanolamine \\
\hline PGC-1 & Proliferator-Activated Receptor-Gamma Coactivator-1 \\
\hline PhiC31 integrase & A sequence-specific recombinase from bacteriophage \\
\hline PI3K & Phosphatidylinositol 3-kinase \\
\hline PIP2 & Phosphatidylinositol 4,5-bisphosphate (PIP2) \\
\hline PKA & Protein Kinase A \\
\hline PLA2 & phospholipase A2 \\
\hline PLC & phospholipase C \\
\hline PLIN1 & perilipin 1 \\
\hline PMCA & plasma membrane calcium ATPase \\
\hline pmol & picomol \\
\hline PMT & photomultiplier \\
\hline POMC & Pro-opiomelanocortin \\
\hline $\mathrm{RdRP}$ & RNA-dependent RNA polymerases \\
\hline Rel. & Relative \\
\hline rRNA & ribosome RNA \\
\hline RISC & RNAi-induced silencing complex \\
\hline RNA & Ribonucleic acid \\
\hline RNAi & RNA interference \\
\hline RNAi On & FB-Gal4/UAS RNAi/CaLexA flies \\
\hline RNAi Off & FB-Gal4/balancer /CaLexA flies \\
\hline RNA-seq & RNA sequencing \\
\hline Rpl3 & Ribosomal protein L32 \\
\hline RT-qPCR & Reverse transcription-quantitative polymerase chain reaction \\
\hline RyR & $\mathrm{Ca}^{2+}$-sensitive ryanodine receptors \\
\hline
\end{tabular}




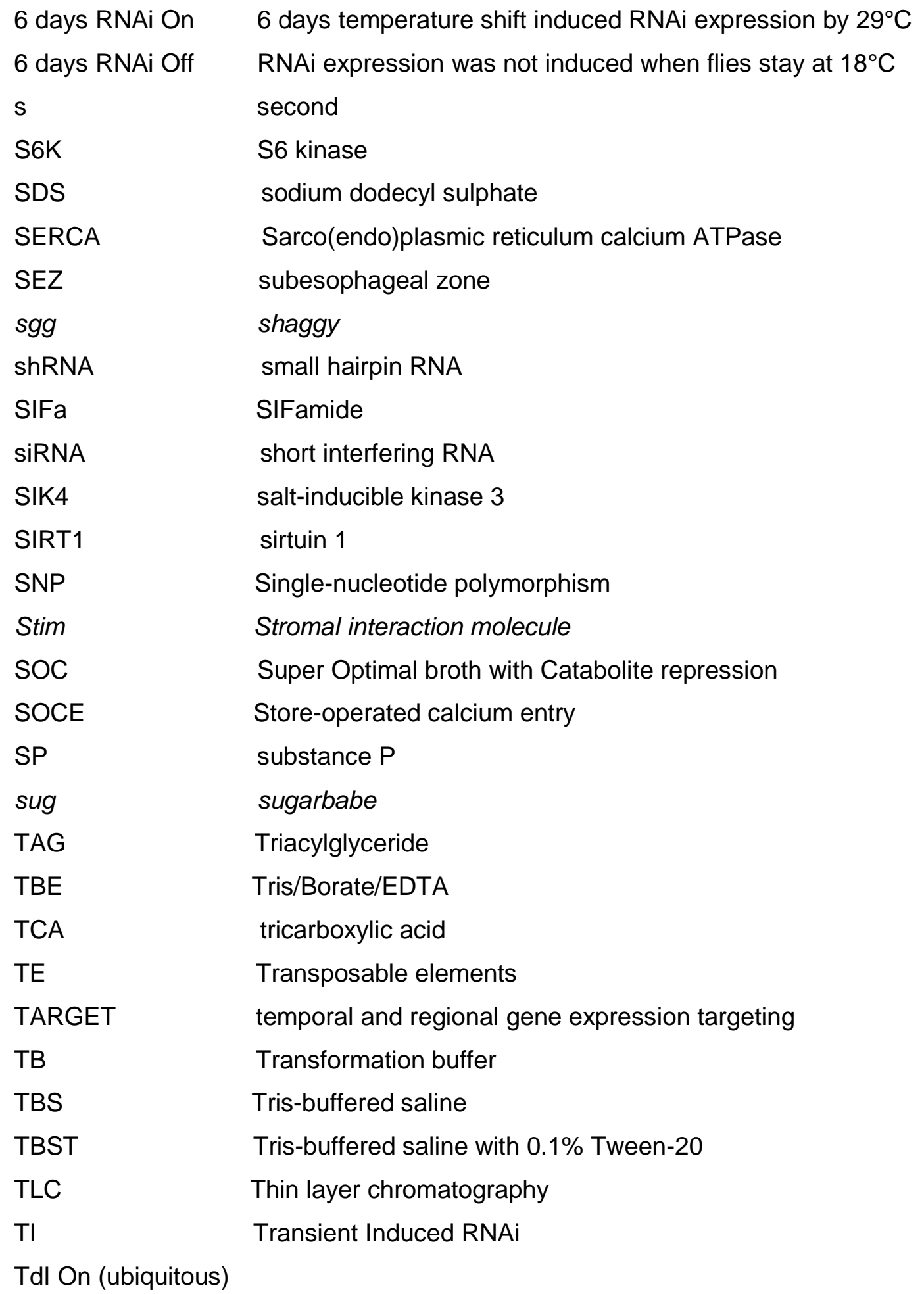

Transient drug-feeding Induced RNAi expression in daGS-

Gal4/UAS-RNAi flies at $25^{\circ} \mathrm{C}$

Tdl Off (ubiquitous)

RNAi expression of daGS-Gal4/UAS-RNAi flies was not Induced when flies were fed with Gö-food at $25^{\circ} \mathrm{C}$

Tdl On negative control 1

Adult offsprings of wild type control crossed with UAS-RNAi1 
flies were transiently fed with drug $25^{\circ} \mathrm{C}$

Tdl Off negative control 1

Adult offsprings of wild type control crossed with UAS-RNAi1

were fed with Gö-food at $25^{\circ} \mathrm{C}$

Tdl On negative control 2

Adult offsprings of wild type control crossed with daGS-Gal4 flies were transiently fed with drug $25^{\circ}$

Tdl On negative control 2

Adult offsprings of wild type control crossed with daGS-Gal4 flies were fed with Gö-food at $25^{\circ} \mathrm{C}$

tobi target of brain insulin

TOR Target of rapamycin

TORC Target of rapamycin complex

Trh Tryptophan hydroxylase

TRiP Drosophila Transgenic RNAi Project

ts-FB-Gal4 temperature sensitive fat body Gal4 driver

Ttl On Transient temperature-shift $\left(29^{\circ} \mathrm{C}\right)$ Induced RNAi expression in ts-FB-Gal4/UAS-RNAi flies

Ttl Off RNAi expression of ts-FB-Gal4/UAS-RNAi flies was not induced when flies stay at $18^{\circ} \mathrm{C}$

Ttl On negative control

Flies only containing a UAS-RNAi transgenic were transiently incubated at $29^{\circ} \mathrm{C}$

Ttl Off negative control

Flies only containing a UAS-RNAi transgenic were kept at $18^{\circ} \mathrm{C}$

TNF Tumor Necrosis Factor

UAS Upstream activation sequence

UAS-Stim RA UAS-Stim cDNA RA

UAS-Stim Rm UAS-Stim RNAi1 resistant cDNA RA modified

$\mu \mathrm{g} \quad$ microgram

$\mu \mathrm{L} \quad$ microliter

upd2 unpaired2

VDRC Vienna Drosophila Resource Center

W Weight

WHO World Health Organisation

Zess LSM Zeiss Laser Scanning Microscopes 


\section{Introduction}

Obesity has become a global pandemic. Therefore, obesity represents a major financial burden and became a major concern in public health intervention programmes. Obesity is defined as the abnormal fat accumulation, which poses risks to human health (Garrow, 1988; WHO Consultation on Obesity, 2000). It is widely assessed by the Body Mass Index (BMI) Weight $/ \mathrm{Height}^{2}\left(\mathrm{~W} / \mathrm{H}^{2}=\mathrm{Kg} / \mathrm{m}^{2}\right)$, first proposed by Quetelet in $18^{\text {th }}$ century, and later introduced by Ancel Keys and his colleagues to define body fat mass in epidemiological studies (Keys et al., 1972). The BMI concept was also supported by a later study (Gray and Fujioka, 1991), and a BMl value $>30 \mathrm{Kg} / \mathrm{m}^{2}$ has been widely used as a conventional criterion for defining obesity in both sexes, even though BMI has certain limitations for more specific and accurate diagnosis (Donini et al., 2013; Mascie-Taylor and Goto, 2007; Pories et al., 2010; Romero-Corral et al., 2008).

Based on BMI, both obesity prevalence of children and adults have substantially increased not only in developed countries but also in developing countries since 1980 (Finucane et al., 2011; $\mathrm{Ng}$ et al., 2014), i.e. the age-standardized global obesity prevalence almost doubled from 6.4\% in 1980 to $12.0 \%$ in 2008 (Stevens et al., 2012). According to World Health Organization (WHO), in 2014 there were over 600 million obese people around the world. To evaluate the health risks of obesity more precisely, other indicators such as waist circumference, waist-to-hip ratio, or waist-toheight ratio were also proposed (World Health Organization, 2008). Obesity, especially abdominal obesity, indicated by waist circumference, has been strongly associated with a list of highly morbid chronic diseases, including metabolic syndrome (Bi et al., 2014b; Després and Lemieux, 2006; Jensen, 2008; Kang et al., 2011; Patel and Abate, 2013; Smith, 2015; Walker et al., 2014), cardiovascular diseases (Britton et al., 2013; Després, 2012; Lim and Meigs, 2013; Neeland et al., 2015), and various types of cancer (Britton et al., 2013; Howe et al., 2013; Incio et al., 2016; Louie et al., 2013). As a consequence, mounting evidences show that there is a significant correlation between obesity and a higher risk of death (Flegal et al., 2013; Petursson et al., 2011; Pischon et al., 2008; Simpson et al., 2007; World Health Organization, 2008).

Obesity prevalence has created enormous economical burdens for both the obese individuals and the whole society. From 1980 to 2002, the obesity-related healthcare burden in Western European countries was estimated as high as $€ 10.4$ billion 
annually and accounted for a range between 0.1 to $0.61 \%$ of each country's gross domestic product (GDP) (Müller-Riemenschneider et al., 2008). Even more alarming is the trend reflected in developing countries: in a Chinese case study, the indirect effects of dietary-induced obesity and lack of physical activity likely contribute to $3.58 \%$ and $8.73 \%$ of the gross national product (GNP) in 2000 and 2025, respectively (Popkin et al., 2006). Moreover, none of these estimates took the weight loss industry into account yet, which reached over $\$ 60$ billion in North America alone (Hruby and $\mathrm{Hu}, 2015)$. Globally, obesity costs about \$2 trillion annually, which corresponds to approximately $2.8 \%$ of world GDP (Blackstone, 2016).

Overall, the wide spread of obesity epidemic has posed a serious challenge to humans. However, over the past decades, no country has been successful in combating the striking prevalence of obesity ( $\mathrm{Ng}$ et al., 2014). Therefore, effective strategies to prevent and treat obesity need to be developed and more research should be conducted to decipher the causes of the disease.

\subsection{The contributors of obesity}

In order to better diagnose obesity and understand its development, research has been focused to identify the factors that contribute to obesity ranging from the physiological to the molecular level. This research was and still is focused at the analysis of energy homeostasis in general on genetic responses to environmental cues.

\subsubsection{Energy balance and obesity}

Eukaryotes, ranging from yeast to man, store excess energy in the form of fat (triglycerides) for future demands (Ottaviani et al., 2011). Accordingly, the excessive body fat in obese people is the result of energy imbalance: energy intake exceeds the energy expenditure (Ravussin and Swinburn, 1992). It is now generally accepted that the proximate cause of obesity is a positive energy balance (Hall et al., 2012; Hill, 2006; Hill et al., 2012; Speakman and O'Rahilly, 2012; Spiegelman and Flier, 2001). Therefore, a more comprehensive understanding of how the energy balance of organisms is regulated will help us to identify contributors of obesity.

The starting point of the energy imbalance theory on obesity is the principle of energy conservation. Biological systems also comply with the first law of thermodynamics, 
i.e. energy can be transformed from one form to another but not created or destroyed (Hall et al., 2012). Since life is dynamic system, the principle of energy conservation was formulated as the dynamic energy-balance equation: the rate of energy stores = the rate of energy intake - the rate of energy expenditure (Hall et al., 2012; Ravussin and Swinburn, 1992).

Normally, energy intake of humans is mainly made up of three major macronutrients: carbohydrate $(4 \mathrm{kcal} / \mathrm{g}, 17 \mathrm{~kJ} / \mathrm{g})$, protein $(4 \mathrm{kcal} / \mathrm{g}, 17 \mathrm{~kJ} / \mathrm{g})$ and fat (the highest one, $9 \mathrm{kcal} / \mathrm{g}, 38 \mathrm{~kJ} / \mathrm{g}$ ). Besides absolute amount, multiple factors including the diet composition, food preparation, and gut microbiome also considerably affect the absorption of ingested nutrients (Hall et al., 2012; Spiegelman and Flier, 2001). The absorbed nutrients need to fuel three kinds of energy expenditure including the activity energy expenditure (i.e. the amount of energy expended by physical activity), the basal resting energy expenditure (i.e. the amount of energy spent at rest) and the thermic effect of food (i.e. the obligatory energy expenditure required by food ingestion and absorption (Hall et al., 2012; Hill et al., 2012; Ravussin and Swinburn, 1992; Spiegelman and Flier, 2001). Activity energy expenditure varies a lot with physical activities; another section of activity energy expenditure comes from the resting energy expenditure, which is associated with body mass, especially with lean tissue; in addition, there is also variability in individuals with respect to the thermic effect of food (which is usually in the range of $8 \%-10 \%$ of total energy output) (Hall et al., 2012; Hill et al., 2012; Ravussin and Swinburn, 1992; Spiegelman and Flier, 2001).

Energy stores occur only if energy intake exceeds the total energy expenditure of the organism. It is the net changes of what carbohydrates, proteins, and fat contribute (Hall et al., 2012). In fact, when positive energy balance happens, body fat accumulation contributes to $60-80 \%$ of body mass increase (Hill and Commerford, 1996). Carbohydrates are stored mainly as glycogen in muscle and liver with rapid turnover rate, and they contribute only to a limited amount of total energy store (only several hundred grams) (Hall et al., 2012). Similarly, daily protein intake account for just around $1 \%$ of the total protein stores in humans (Ravussin and Swinburn, 1992). Besides, it seems that the glycogen and protein stores are under a tightly regulated balance (Ravussin and Swinburn, 1992). The metabolism of glycogen and protein are additionally involved in water changes of the organism, which also cause body weight changes (Hall et al., 2012). Therefore, in addition to measuring energy intake 
and energy expenditure, body fatness instead of body weight may more likely represent energy imbalance (Hall et al., 2012; Ravussin and Swinburn, 1992).

There has been a long and intensive debate on which component of the energy equation contributes most to the imbalance (Hill et al., 2012; Speakman and O'Rahilly, 2012). It appears to be widely accepted that increased food supply at population level can sufficiently explain obesity epidemic (Swinburn et al., 2009; Vandevijvere et al., 2015). More specifically, increased sugar intake and fast food was found to be associated with weight gain and obesity risk (Te Morenga et al., 2013; Rosenheck, 2008). However, the methods (i.e. personal questionnaire or recall) which were used to quantify food intake might be vulnerable to inaccurate reports (Bandini et al., 1990; Dhurandhar et al., 2015). In addition to blaming the food consumption as a sensible cause of obesity, researchers also argue that sedentary lifestyle (such as television watching) has been strongly associated with the spread of obesity epidemic (Castañeda et al., 2005; Church et al., 2011; Hu et al., 2003; Livingstone et al., 2003; Roda et al., 2016). But despite the conclusions that can be drawn from such data and ongoing research, no significant decrease has been observed with respect to human daily energy expenditure since the 1980s (Westerterp and Speakman, 2008). An interesting observation is that the energy expenditure of obese people is higher than in their lean counterparts (Schoeller, 2008). Hill et al (Hill et al., 2012) argue that the primary cause of obesity is the declined physical activity levels, which results in an increased body weight, the following increased basal resting energy expenditure and the increased energy cost of movement (Hill et al., 2012). In fact, many researchers support the notion that studies on energy intake or energy expenditure alone are incomplete, and that obesity research should put more emphasize on the mismatch between energy intake and expenditure as well as its underlying mechanisms (Hill et al., 2012; Schoeller, 2008).

It is important to note that energy stores, energy intake, and energy expenditure are closely inter-connected and inter-dependent (Ravussin and Swinburn, 1992). Ample evidences show that components of energy balance can interact with each other, i.e. the food composition affect energy store and expenditure (Bray and Popkin, 1998; van Dam and Seidell, 2007; Dirlewanger et al., 2000; Horton et al., 1995), energy expenditure in turn can interact with appetite control (Blundell et al., 2012, 2015) and the energy store can also regulate the food intake (Hall et al., 2011) as well as energy expenditure (Schoeller, 2008). It has also been reported that the complex 
interactions among the various components of the energy balance system are regulated by a sophisticated physiological control system, otherwise human body weight would be easily swayed by short-term energy related behaviors (Hill et al., 2012).

\subsubsection{Gene, environment and interaction}

But if the energy control system always favor for well-balanced energy homeostasis, how could the control system allow for an alarming obesity pandemic. In fact, it is justifiable that energy balance control system exists, as losing weight is much more difficult than gaining weight (Hill et al., 2012). Moreover, the body's ability to buffer energy imbalance is limited (Hill et al., 2012), and it varies from person to person under the current conditions (Speakman and O'Rahilly, 2012; Spiegelman and Flier, 2001). Therefore, it is essential to learn which genetic factors govern the energy balance control system, and how environmental factors interact with these genetic factors and cause continued energy imbalance in millions of individuals.

It has been known since some time that genetic factors play a pivotal role in obesity. Based on identical and fraternal twin studies, around $40-70 \%$ of obesity can be attributed to inherited genetic variances (Barsh et al., 2000; Comuzzie and Allison, 1998; Farooqi and O'Rahilly, 2000). A strong correlation between obesity and genetic background was also found in an adoptive children study (Stunkard et al., 1986). In fact, people have successfully identified less than a dozen of monogenic obesities (Xia and Grant, 2013). The best known example is the discovery of the leptin gene, which encodes an adipose tissue hormone, which acts as a food intake inhibiting signal in the brain (Halaas et al., 1995). As early as in the 1950s, an obese mutation ob/ob in mice was reported (Ingalls et al., 1950); but its function remained puzzling for decades until the identification of the mutation localized at leptin gene in 1994 (Zhang et al., 1994). Shortly afterwards, another famous obese mutation in mice, $d b / d b$, a mutation in the leptin receptor was also identified (Chua et al., 1996). Moreover, leptin and leptin receptor mutations in humans were found later (Clément et al., 1998; Montague et al., 1997). In addition, mutations in other notable genes including POMC (encodes pro-opiomelanocortin) and MC4R (encodes melanocortin 4 receptor) were also identified and reported to cause Mendelian obese phenotypes in rodents and humans (Barsh et al., 2000; Bell et al., 2005; Xia and Grant, 2013). 
Moreover, to study genetic risks of common polygenic obesity, with the help of powerful genome-wide association studies (GWAS) analysis, researchers directly compare genomes of a large number of obese and lean individuals, thereby associating more broad genetic variants (majorly in form of single-nucleotide polymorphisms (SNPs)) to obesity-related phenotypes (Willyard, 2014). For example, a GWAS study in 2007 found that individuals carrying two copies of a common genetic variant in the FTO (Fat Mass And Obesity Associated) gene are on average $3 \mathrm{~kg}$ heavier than normal persons (Frayling et al., 2007). Interestingly, the obesityassociated FTO variant functionally gets involved in the regulation of IRX3's gene expression, which further directly affects body weight via reduction of body mass and increase of basal metabolic rate (Smemo et al., 2014). The none-hypothesis-driven nature of GWAS has successfully enabled researchers to uncover many more new obesity-related genetic factors: 141 candidate obese loci have been identified so far (Levian et al., 2014). However, common genetic polymorphism discovered by GWAS account for only about $10 \%$ of estimated obesity heritability or even less (Levian et al., 2014; Willyard, 2014; Xia and Grant, 2013). Although Studies on these genes have provided some insights into the biological mechanisms underlying energy homeostasis and obesity, there are still dark matter with respect to the understanding of genetic heritability of obesity, e.g. rare genetic variants or collective effects (Levian et al., 2014; Willyard, 2014; Xia and Grant, 2013). Furthermore, since genetic variants obtained through GWAS are merely correlation (Xia and Grant, 2013), the causal links between an impaired key energy balance genes and obesity are not established yet.

Although one cannot deny the role played by genetic factors in obesity development, a relatively small difference of the Body Mass Index (BMI) due to known high genetic risks does not match with the drastic global obesity pandemic over the past several decades, which points to obesity risk factors beyond genetics (Hruby and Hu, 2015). Importantly, given frequent food scarcity, high levels of physical activity, increase of the brain size as well as an expansion of immune system and reproduction demand during the course of human evolution, both an elaborate and complex physiological system and a corresponding genetic system needed to efficiently accumulate energy through stored fat for survival has evolved, which by now inherently poses higher risks of obesity to humans in the current obesogenic environment (e.g. high availability of calorie-dense and palatable food, wide use of labor-saving devices) (Bellisari, 2008; Wells, 2012). Therefore, alternative and/or additional possible obesity drivers are obesogenic environmental factors, which coincidently have 
changed during the period when the upward trend of global obesity rate became apparent (Swinburn et al., 2011). More specifically, the obesogenic environmental factors include proximal determinants of individual behaviors (abundant, inexpensive, high fat, high sugar food supply, labor-saving devices and automatic building environments, xenobiotic chemicals or antibiotics, stressful lifestyles, etc.), and distal systemic drivers (industrialization and urbanization; economic policies such as taxation or sugar-sweetened beverages marketing; the widening gap between the poor and the rich; cultural preferences etc. (Cox and Blaser, 2015; Hruby and Hu, 2015; Levian et al., 2014; Swinburn et al., 2011). Furthermore, an increasing number of studies indicate that proximal obesogenic environmental factors, likely to be epigenetic regulators of gene transcription (non DNA sequence change e.g. DNA methylation, histone modification, chromatin remodeling, and non-coding RNAs), result in lasting effects during the entire life span of people and even increase the obesity risk of the offspring (Hruby and Hu, 2015; Levian et al., 2014; Xia and Grant, 2013). Concerning the so far unsuccessful efforts to reverse the global obesity epidemic, the promising answer to the alarming problem likely not lies in only identifying different risk factors, but rather in learning how they relate to genetic predispositions of individuals, whether and how they cause intricate network feedback loops at systems-levels which ultimately lead to obesity (Hruby and $\mathrm{Hu}$, 2015; Levian et al., 2014).

Overall, genetic and gene-environmental studies have offered us a great deal of knowledge on monogenic, polygenic as well as environmental risk factors. Despite the progress, possibly a large proportion of causal genetic contributors remains unknown. In addition, it is the complex interactions among genes, signaling pathways, physiological processes, and organs of energy regulatory networks contribute to human inherent limitations in adaptive capability to offset environmental challenges in obesity. Hence, we need a better understanding of energy regulatory networks in addition to individual genetic factors.

\subsection{Drosophila melanogaster as an emerging model for obesity research}

To get a full picture of the dynamic energy regulatory networks, Drosophila melanogaster, the fruit fly, has emerged as an attractive model to carry out energy metabolism studies. In the following, I will describe the key features of this model organism and its use in obesity research. 


\subsubsection{Powerful model system}

Since Drosophila melanogaster was first proposed as a genetic model organism in 1900 (Davenport C. B., 1941; Sturtevant A. H., 1959), we have witnessed a list of remarkable discoveries on the fundamental mechanisms of biology. They include Morgan's work on the role of chromosome in heredity, the characterization of essential signaling pathways in development and cell biology, and discovery of activating immune responses in Drosophila (Markow, 2015; Rieder and Larschan, 2014).

Although the fly genome only consists of 4 chromosomes and encodes about 14,000 genes (Adams et al., 2000; Attrill et al., 2016), there are about 65\% of human disease genes, which can be identified as Drosophila homologs in the genome of fly (Chien et al., 2002; Reiter et al., 2001; Yamamoto et al., 2014). In fact, the reduced complexity of fly as compared to mammalian genome facilitates studies of basic molecular mechanisms that are much more difficult to unravel in vertebrates due to functional redundancies (Rieder and Larschan, 2014). In addition, when flies were grown on normal food containing corn flour, soybean flour, yeast, as well as various sugar and preservatives, it takes only $9-10$ days at $25^{\circ} \mathrm{C}\left(18-19\right.$ days at $\left.18^{\circ} \mathrm{C}\right)$ for a fertilized egg to develop into adult fly. Subsequently, virgin female flies can be selected within 8 hours after eclosion with $\mathrm{CO}_{2}$ (newly eclosed virgin females have unopened wings, and pale abdomen with a green dot), mated to males with the selected genotypes in a fresh food vial where they mate and lay fertilized eggs on the surface of food. After one day, $1^{\text {st }}$ instar larvae hatch and burrow in the food. They undergo two moltings ( $1^{\text {st }}$ instar larvae hatch from eggs and burrow into the food, who later enter sequentially enter $2^{\text {nd }}$ instar at day three, and become $3^{\text {rd }}$ instar at day four), (after three and four days, respectively) after which they crawl out of food and stop eating. During the days five-nine, larvae form pupae and undergo metamorphosis, and adult flies finally hatch from the puparium (Hales et al., 2015). This short life cycle of flies speeds up discoveries (Hales et al., 2015; Markow, 2015).

Moreover, being studied for more than a century, extensive genetic tools have become available for gene function studies: e.g. balancer chromosomes (Hales et al., 2015; Muller, 1918), transgenic lines by transformation(Venken and Bellen, 2007), conditional binary Gal4/UAS gene expression systems (Brand and Perrimon, 1993; McGuire, 2003), and clonal analysis (Golic and Lindquist, 1989; St Johnston, 
2002; Theodosiou and $\mathrm{Xu}$, 1998). Balancer chromosomes are engineered chromosomes containing visible marker mutations and multiple inversions that prevent recombination events. Since flies, which are homozygous for a balancer chromosome, are lethal, the use of balancer chromosomes allows for the selection of offspring that carries the parents' one copy of a certain chromosome containing the mutation of interest, as well as the maintenance of stable heterozygous mutation lines (Greenspan, 2004; Hales et al., 2015). The generation of transgenic Drosophila took advantage of the transposable P-element as germ-line transformation vector (Rubin and Spradling, 1982). The P-element transgene contains the modified transposable element including the gene of interest. Its DNA and an independent source of transposase enzyme are co-injected into syncytial blastoderm embryos. Subsequently, transposase enzyme activity causes random inserts of the transposable element into genome of the developing germline (Klemenz et al., 1987; Rubin and Spradling, 1982). Later improvements of the system such as the more efficient PhiC31 integrase system has been widely used for targeted insertions. This system uses flanking attB sequences which direct the transgene to a specific landing site with attP sequence within the genome (Bischof et al., 2007; Groth et al., 2004; Venken and Bellen, 2007). In order to activate the transgene in a spatial/temporal manner, tissue-specific or cell type specific promoter/enhancer controlled-Gal4 transcription factor - UAS- gene of interest expression systems were generated, in which tissue-specific or cell type specific promoter/enhancer controlled Gal4 transcription factor binds to the upstream activating sequence (UAS) and activates the expression of a gene of interest (called as UAS transgenic lines) (Brand and Perrimon, 1993). Moreover, the binary Gal4/UAS system was combined with temperature-sensitive Gal4 repressor-Gal80 ts which then allows for temporal and regional gene expression targeting (TARGET) in Drosophila (McGuire, 2003). Furthermore a drug (mifepristone)-inducible tissue-specific Gal4 system was developed which acts also in combination with UAS transgenic lines (Roman et al., 2001; Tricoire et al., 2009). Information about fly lines and Drosophila genes and fly stocks can be obtained from fly stocks collection centers (Bloomington Drosophila Stock Center, BDSC, http://flystocks.bio.indiana.edu/, Vienna Drosophila Resource Center, VDRC, http:// stockcenter.vdrc.at/control/main) (Dietzl et al., 2007), and the bioinformatics platforms like FlyBase (Attrill et al., 2016).

Furthermore, to manipulate gene expression, abundant fly strains in above mentioned fly stock centers have been generated based on three major genetic strategies: (1) gene mutation, (2) a wild type or a mutant version for cDNA transgene 
overexpression, and (3) gene knockdown via RNA interference (RNAi) (Ugur et al., 2016). Mutations can be generated either at random by using X-ray radiation, by chemical treatment (e.g. Ethyl methanesulfonate, EMS), or by targeted disruption, e.g. traditional transposon-mediated mutagenesis and excision of existing transposable elements (TE) (St Johnston, 2002). The latest development in generating mutants includes the clustered regularly interspaced short palindromic repeats (CRISPR)/Cas9 (CRISPR associated protein 9) technique to generate sequence-specific "designer mutants" (Kondo and Ueda, 2013). Transgene overexpression approaches can be used for "rescue experiments" to complement a loss-of-function mutation in order to confirm a sequence-to-function relationship. In addition, they can be used for loss-of-function studies in which the dominant negative form of a cDNA transgene is overexpressed. Moreover, overexpression or ectopic expression of genes help to establish gain-of-function disease models (Ugur et al., 2016).

In my project, I have deployed the above mentioned RNAi gene knockdown technique as an effective agent to lower the mRNA levels of distinct target genes, thereby reducing or impairing gene product functions (Hales et al., 2015). RNAi was first discovered in the nematode worm Caenorhabditis elegans (C. elegans) as sequence-specific gene silencing in response to exogenous double-stranded RNA (dsRNA) (Fire et al., 1998; Montgomery et al., 1998). Subsequently, this approach was shown to also function in fungi, animals and plants (Hannon, 2002; Mello and Conte, 2004). Biochemical studies on Drosophila embryo and cells made a substantial contribution to understand the underlying mechanism of RNAi-mediated post-transcriptional gene silencing (Daneholt, 2006; Hannon, 2002). For example,

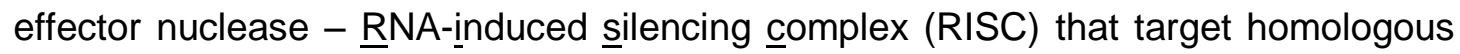
mRNAs for degradation was identified from Drosophila S2 cells (Hammond et al., 2000). Similar to what has been observed in plants, short interfering RNAs (siRNAs) (21-23 nucleotides) that guide mRNA degradation could be produced from exogenous dsRNAs in Drosophila embryo extracts (Zamore et al., 2000). Over the years, the basic mechanism of RNAi has been deciphered. Exogenous dsRNAs are the primary trigger, which are processed into siRNAs by the endonuclease Dicer. The antisense RNAs of siRNAs are joined with the RISC complex, whereby they guide the complex to the corresponding mRNA strand via base-pairing. Finally, the mRNA strands are cut by the RISC complex and are subsequently degraded (Daneholt, 2006; Hannon, 2002). Despite the core mechanism of RNAi is conserved, there are differences between RNAis of various organisms. For instance, RNAi in 
C.elegans can spread across the worm's tissues and from a parental animal to its offspring, while RNAi in Drosophila and mammals apparently acts only cell autonomous and in a non-heritable fashion (Hannon, 2002; Van Roessel et al., 2002; Roignant et al., 2003). Therefore, unlike RNAi in C.elegans, the effects of transfecting siRNA into Drosophila and mammal cells or injecting siRNA into animals are both local and transient (Perrimon et al., 2012). Further, to achieve long-term gene silencing effects, small hairpin RNAs (shRNAs) or long hairpin RNA synthesizing vectors were used to transform into the genome of cells or animals to enable continuous production of processed siRNAs from hairpin RNAs (Hales et al., 2015; Hannon, 2002; Perrimon et al., 2012). More importantly, the combination of Gal4 driver controlled by specific enhancer or promoter and UAS-shRNA or long hairpin RNA transgenes for RNAi (UAS-RNAi) allows for the reduction of specific mRNAs in a precise tissue and temporal specific pattern (Brand and Perrimon, 1993; Kennerdell and Carthew, 2000; Perrimon et al., 2012).

Since the genome sequences and genes of Drosophila have been identified at the beginning of genome sequencing era (Adams et al., 2000; Attrill et al., 2016), numerous genome-wide UAS-RNAi libraries have been constructed, which can be utilized to target at each gene of the genome (Vienna Drosophila RNAi Center (VDRC, http:// stockcenter.vdrc.at/control/main; Drosophila Transgenic RNAi Project (TRiP) at Harvard Medical School (http://www.flyrnai.org/TRiP-HOME. html) (Dietzl et al., 2007; Ni et al., 2008)(Perkins et al., 2015). Collectively, these libraries allow for high throughout RNAi screens to target about $90 \%$ of the protein coding genes of the fly genome with respect to phenotypes of interest (Hales et al., 2015).

To date, with the help of well-characterized Gal4 driver lines, transgenic RNAi lines have been shown to be effective in different somatic tissues at specific life stages (Perrimon et al., 2012). The power of in vivo RNAi screens has been used to identify new regulator genes of energy homeostasis. These genome-wide obesity screens with Drosophila identified Hedgehog as an essential factor which acts in determining adipocyte cell fate (Pospisilik et al., 2010) and, in a fat storage tissue (fat body tissue and midgut)-specific screen in in adult Drosophila, identified the store-operated calcium entry (SOCE) as key regulator of adiposity (Baumbach et al., 2014a). Both of these screens confirmed the utility of the fly as an attractive biological system for obesity research from which valuable insights on energy homeostasis control can be expected. 


\subsubsection{Energy metabolic organ systems in Drosophila and human}

Given the evolutionary conservation of genes and tractable genetic systems in fruit fly, Drosophila is not limited to traditional genetics and the development field where it has continuously be used over more than a century. It has recently emerged as powerful model organism for human diseases studies (Bellen et al., 2010; Ugur et al., 2016) such as energy metabolism and obesity (Padmanabha and Baker, 2014; Rajan and Perrimon, 2013; Smith et al., 2014). More importantly, the major reason for using of Drosophila as an attractive model to study obesity is the significant similarities between the fly and human organ systems (Padmanabha and Baker, 2014; Rajan and Perrimon, 2013; Trinh and Boulianne, 2013).

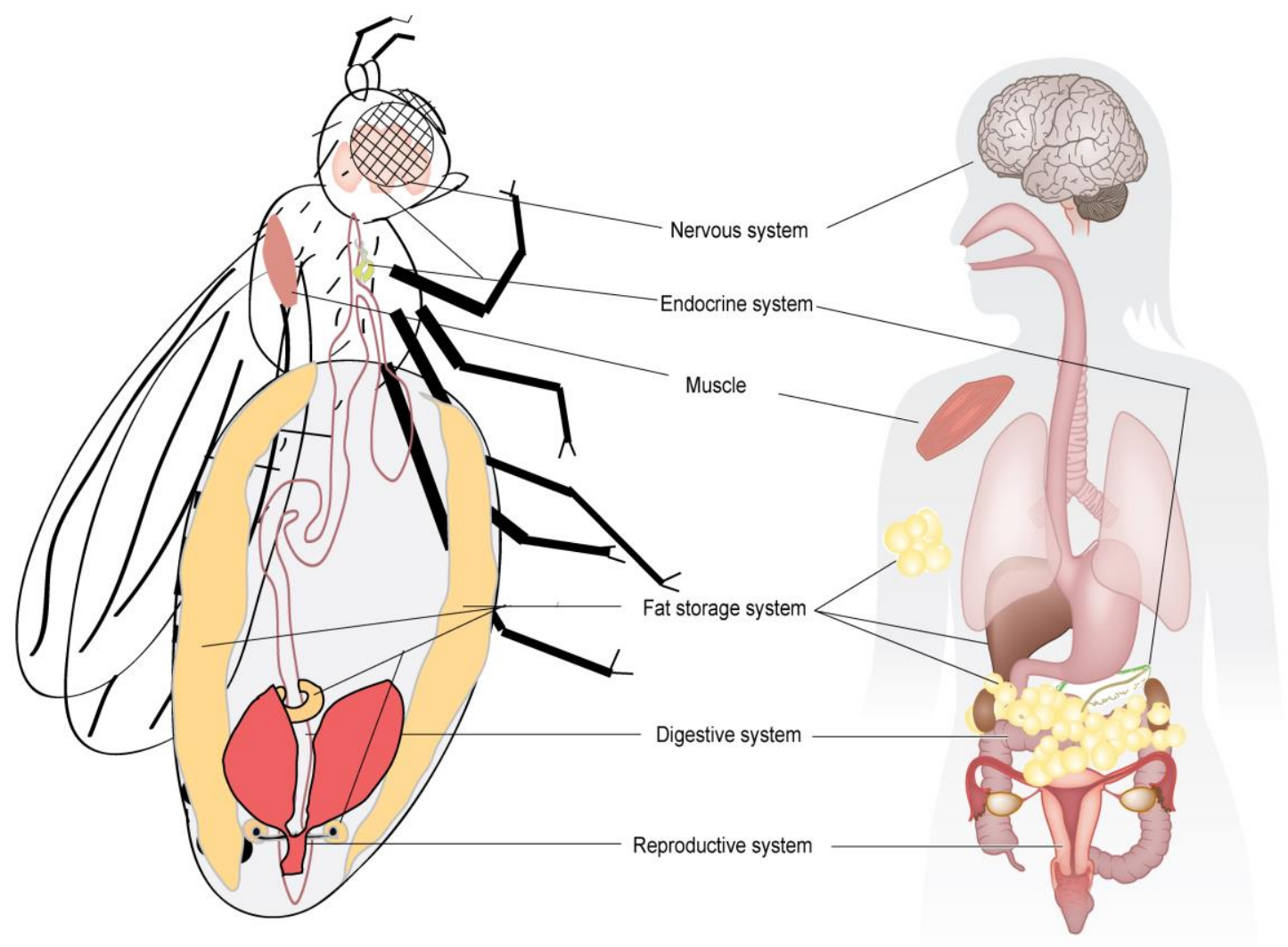

Figure 1. The organ systems of Drosophila melanogaster are functionally analogous to those in human

First, Drosophila melanogaster central nervous system is functionally equivalent to the brain in the human. Second, Drosophila has neural endocrine cells in located in the brain (Insulin Producing cells, not illustrated), and located in corpora cardiaca (light green structure), which are analogous to the pancreas of the human. Third, muscle system is similar between fly and human. Fourth, fat body tissue (containing cuticle attached fat body tissues, gut ring fat body tissue, and spermatheca attached fat body tissues in female flies) and oenocytes (attached to cuticle fat body tissue, not illustrated) in Drosophila are functionally equivalent to the human adipose and liver tissues (fat storage system). Moreover, both fly and human have similar digestive system (gut) and reproductive system (female ovary system as example). The fact enables us to study the energy regulatory networks in this powerful model system. The figure 
is made with Adobe Illustrator and Pathvisio (https://www.pathvisio.org/) based on figures in (Buchon et al., 2014) and (Droujinine and Perrimon, 2016).

Most of the internal organ systems of adult flies are functionally analogues to their vertebrate counterparts, including humans, which have similar metabolic processes (Padmanabha and Baker, 2014; Ugur et al., 2016) (please see Figure 1). The fly takes in food, digests and absorbs the nutrients in a segmented digestive tract (midgut) and temporally stores limited amount of triacylglyceride, which functions similar to the mammalian stomach and intestine (Apidianakis and Rahme, 2011; Pitsouli and Perrimon, 2008). The loosely organized and dispersed fat body tissue of the fly is a major organ for energy reserve (glycogen and triacylglyceride), which is the functionally equivalent organ of white adipose tissue in mammals (Kühnlein, 2011; Padmanabha and Baker, 2014). Midgut and fat body tissue together are called as fat storage tissue in Drosophila (Baumbach et al., 2014a). Moreover, together with oenocytes, fat body cells can also serve as the site of energy metabolism in response to starvation, acting as functionally equivalent to the human liver (Gutierrez et al., 2007; Padmanabha and Baker, 2014). The released energy molecules are utilized by other organs, e.g. muscle and heart, gonads, brain or developing organs (Droujinine and Perrimon, 2016). Furthermore, it has been found that fat body tissue serves as an endocrine organ, which interacts with other tissues e.g. gut and brain to coordinate different biological processes e.g. energy metabolism, immune responses, growth and circadian rhythms by sensing, integrating, and secreting different cytokines, lipid mediators (Buchon et al., 2014; Dionne, 2014; Droujinine and Perrimon, 2016; Hotamisligil, 2017; Liu et al., 2014a). To adapt to feeding and fasting, the corresponding behavior is directly controlled by brain cell activities. The brain of the fly is also the location of the insulin producing cells (IPCs), which coordinate the energy metabolism, whereas the functional homolog of IPCs in vertebrates are pancreatic $\beta$ cells (Wu and Brown, 2006). Furthermore, the corpora cardiaca contains also a group of neurosecretory cells with a similar function to the pancreatic $\alpha$ cells of vertebrates (Droujinine and Perrimon, 2016).

\subsubsection{Energy reserve storage in Drosophila and human}

Normally, dietary sugar and lipid digestion play a key role in lipid storage. In terms of sugar absorption, glucosidases and other enzymes in the fly gut break down the dietary sugars into monosaccharides such as glucose, which are transferred into the hemolymph, which represents the Drosophila equivalent to the vertebrate circulatory system, by gut enterocytes. From there, the fat body tissue takes up the 
monosaccharides and uses them to synthesize the glycogen store from where it is released in form of trehalose into hemolymph. Trehalose represents the circulating sugar used by tissues, or is converted into the triacylglycerides (TAGs), the fat storage of the fly (Chng et al., 2016; Turunen and Crailsheim, 1996). Dietary lipids, mainly triacylglycerides (TAGs), are broken down into free fatty acids (FAs) and monoacylglyceride (MAG) by lipases in the gut lumen (Sieber and Thummel, 2009; Trinh and Boulianne, 2013). MAGs and FAs are transformed into TAGs again, which are transiently stored in lipid droplets (LDs) of midgut cells (Sieber and Thummel, 2009). Other absorbed lipids get metabolized and are transported for energy production from gut to the various tissues or to fat body tissue. This transfer involves the ApoB (Apolipoprotein)-family lipoprotein lipophorin (Lpp). Lpp, with the help of another ApoB family member lipoprotein-lipid transfer particle (LTP), is in charge of diacylglycerides (DAGs) transport in Drosophila (Carvalho et al., 2012; Palm et al., 2012).

Flies fed with either high sugar or high fat diet will store excessive fat in fat body tissues, which is a major driver of fly obesity (Birse et al., 2010; Gilby, 1965; Musselman et al., 2011). After excess food intake, fly fat body tissue mainly stores extra nutrients (sugar, lipids or amino acids) as triacylglycerides (TAGs) in an unique intracellular organelle, the lipid droplets (LDs) (Birse et al., 2010; Kühnlein, 2012; Musselman et al., 2011). LDs consists of a hydrophobic core composed of neutral lipids including TAGs and sterol esters, which is surrounded by a monolayered amphipathic membrane formed by phospholipids (e.g. phosphatidylethanolamine (PE), phosphatidylcholine (PC)) and proteins (Krahmer et al., 2011; Walther and Farese, 2012). Lipid droplets are ubiquitous, complex dynamic organelles in charge of lipid storage and lipid supply which are conserved from yeast to mammals (Kühnlein, 2012; Walther and Farese, 2012). Recently, a model about the de novo biogenesis and the origin of the LDs has been proposed: after neutral lipid biosynthesis, TAGs accumulate in the intra-membrane space of the endoplasmatic reticulum (ER) bilayer where they form a "lense"; this lense expands and forms the LD. LDs grow subsequently in size and are released from or remain attached to the ER (Pol et al., 2014; Tan et al., 2014; Wang et al., 2016a; Wilfling et al., 2014).

\subsubsection{Neutral lipid biosynthesis}

De novo TAG synthesis is essential for lipid droplet biogenesis. It requires the coordination of the following steps. Synthesis of fatty acyl-CoA, the de novo synthesis of diacylglycerols (DAG), phospholipid synthesis involving the Kennedy 
pathway and the remodeling of the phospholipids via the Lands cycle (Pol et al., 2014). Each step of the lipid synthesis also interdepends on other metabolic pathways such as fatty acid synthesis, the pentose phosphate pathway, glycolysis, etc. (Chng et al., 2016; Laplante and Sabatini, 2009). In a final step, one acylCoA:diacylglycerol acyltransferases (DGAT) encoded by the Drosophila gene midway ( $m d y$ ) has been identified to catalyzes the synthesis of TAG by adding a fatty acyl-CoA to the DAG molecule (Kühnlein, 2012; Pol et al., 2014).

Fatty acyl-CoA is produced through esterification of fatty acids with coenzyme $A$ (CoA), a synthesis step that is catalyzed by acyl-CoA synthetase (ACS) enzymes (Wilfling et al., 2014). FAs are either taken up exogenously or become endogenously synthesized (Keith, 1967; Wilfling et al., 2014). In FA synthesis, the rate limiting step is the carboxylation of Acetyl-CoA to form malonyl-CoA by the acetyl-CoA carboxylase (ACC), which finally generates fatty acids by fatty acidy synthetase (FAS) (Linn and Srere, 1980; Renobales and Blomquist, 1994; Soulie et al., 1984). Acetyl-CoA is mainly produced in the mitochondrial matrix by different but interdependent metabolic pathways involving glycolysis (i.e. pyruvate is decarboxylated by pyruvate dehydrogenase complex to generate acetyl-CoA) and $\beta$ oxidation (i.e. ATP citrate lyase catalyzes the synthesis of Acetyl-CoA from citrate). In addition, it involves the catabolism of branched amino acids, and acetate can also be used directly by acyl-CoA synthetase (ACS) short chain family enzymes in cytoplasm to generate acetyl-CoA (Pietrocola et al., 2015), which is further converted into long chain fatty acetyl-CoA by Acyl-CoA synthetase long-chain (ACSL) (Huang et al., 2016; Liu et al., 2014b). Recent studies show also that dietary sugar can upregulate the expression of key lipogenic genes e.g. acetyl-CoA synthetase (ACS), fatty acidy synthetase (FAS), the glia-similar transcription factor ortholog gene sugarbabe (sug) (the sugarbabe-dependent target genes include key de novo lipogenes gene e.g. ACS) (Mattila et al., 2015) as well as the Activin ligand gene dawdle (daw). These genes mentioned above were found to be regulated by sugar responsive transcription factor complex Mondo-Mlx (Chng et al., 2016; Mattila et al., 2015).

In Drosophila, DAGs might also be de novo synthesized in addition to being provided directly from the midgut tissue. At the initial step, glycerol-3-phosphate which derives from glycolysis is converted to lysophosphatidic acid (LPA) by acylation catalyzed by the glycerol phosphate acyltransferase (GPAT), likely to be encoded by the CG5508 Drosophila gene (Kühnlein, 2012). In the second step, lysophosphatidic acid (LPA) is 
further converted to phosphatidic acid (PA) through the second acylation catalyzed by 1-acyl-sn-glycerol-3-phosphate O-acyltransferases (AGPAT), likely to be encoded by fu12 which is expressed in fly fat body tissue (Kühnlein, 2012). Finally, once phosphatidic acid (PA) is generated, it will be used to generate DAG by $\mathrm{Mg}^{2+}$ dependent PA phosphatases (PAP1), which is encoded by a single mammalian lipin homologous gene Lipin (CG8709) in Drosophila (Kühnlein, 2012; Ugrankar et al., 2011). Lipin is known to be the rate-limiting enzyme for DAG and TAG synthesis (Han et al., 2006; Ugrankar et al., 2011).

At the same time, the Lands cycle of phospholipid remodeling also contributes to the de novo synthesis of DAG. It involves lysophosphatidic acid (LPA) which can be generated from 2-lysophosphatidylcholine (LPC) by lysophospholipase D (Lyso-PLD) (D'Arrigo and Servi, 2010; Pol et al., 2014), and 2-lysophosphatidylcholine (LPC) which is generated from phosphatidylcholine (PC) by phospholipase A2 (PLA2) (Steinhauer et al., 2009). In addition, phospholipase D might also give rise to phosphatidic acid (PA) by converting PC (Carrasco and Mérida, 2007). Moreover, PA promotes activities of CTP:phosphocholine cytidylyltransferases (CCTs) which use PA and choline to synthesize CDP-Choline, which is rate limiting for PC synthesis (Cornell and Ridgway, 2015; Krahmer et al., 2011). Last but not least, DAGs can also participate in the PC and PE synthesis, which is catalyzed by the CDP choline:diacylglycerol choline-phosphotransferase (CPT) (Krahmer et al., 2011) and CDP-ethanolamine phosphotransferase (encoded by bbc in Drosophila), respectively (Kühnlein, 2012; Lim et al., 2011). Therefore, lipin promotes TAG synthesis while repressing membrane phospholipids synthesis, which represents an alterable switch leading to the synthesis of either neutral lipids or membrane phospholipids.

\subsubsection{Lipid droplet (LD) biogenesis}

It appears that the formation of LDs, containing neutral lipids, is initiated by a low level accumulation of TAGs between the leaflets of the ER bilayers (Wilfling et al., 2014). As the acyl-CoA:diacylglycerol acyltransferases (DGAT) for TAG is distributed evenly on the ER membrane, new synthesized TAGs are proposed to accumulate at certain spots and cause lens formation (Wilfling et al., 2014).

\subsubsection{LD formation, growth and expansion}

After the accumulation of neutral lipids reaches a certain size, meaning it forms a lens, the nascent LDs $(<200 \mathrm{~nm})$ are thought to bud from ER and continue to expand their volume until they finally separate from ER membrane. The fat storage-inducing transmembrane protein (FITM/FIT), likely encoded by CG10671 of the fly, is in fact 
located in ER and is supposed to participate in LD budding (Gross et al., 2010; 2011). In addition, several studies have recently shown that the protein Seipin can stabilize the ER-LDs contact site and thereby facilitates the incorporation of protein and lipid cargos into the growing LDs, a step which is critical for the conversion of nascent LDs into mature LDs (300-500 nm) (Grippa et al., 2015; Salo et al., 2016; Wang et al., 2016a). Further LD expansion in volume depends on the TAG synthesis, which occurs directly at the LDs and an extension of the phospholipid single layer membrane that surrounds the core of neutral lipids. TAG synthesis enzymes such as the GPAT can relocate to the LDs membrane to promote additional LD growth (Wilfling et al., 2013). Limiting phosphatidylcholine (PC) increase the surface tension of LDs membrane, which also participates in the coalescence process of two or several LDs (Pol et al., 2014). The PC content of LDs membrane is thought to be regulated by LD-associated activities of localized CCTs, which is encoded by CCT1, and lysophosphati-dylcholine acyltransferase (LPCATs 1/2), which is encoded by CG32699 (Krahmer et al., 2011; Moessinger et al., 2014). Additionally, in the mammalian adipocyte, large LDs can be generated via a transfer of TAGs from a small LD to an adjacent larger LD ("permeation process") (Wilfling et al., 2014).

\subsubsection{Mobilization of the energy storage in Drosophila and human}

In Drosophila, the glycogen store is mobilized by the enzyme glycogen phosphorylase to meet the rapid energy demand of the organism. The mobilization product is mainly trehalose, a disaccharide formed from two glucose moieties, which circulates subsequently in hemolymph (Arrese and Soulages, 2010; Matsuda et al., 2015). Consistently, the glycogen level decreases in response to starvation treatment of the organism (Baumbach et al., 2014a; Choi et al., 2011) and the resulting trehalose is subsequently used as energy substrate in muscles as well as in other organs by its break down followed by glycolysis (Reyes-DelaTorre et al., 2012). During glycolysis, from one molecule glucose (six carbon sugar), 2 three-carbon molecules-pyruvate, 2 molecular units of energy transfer - adenosine triphosphate (ATP) (energy currency), and 2 reduced electron carrier molecules-nicotinamide adenine dinucleotide (NADH) were generated. Pyruvate is transported into the mitochondria, where it is catalyzed into Acetyl-CoA and $\mathrm{CO} 2$ by the pyruvate dehydrogenase complex. Acetyl-CoA is a substrate for the tricarboxylic acid (TCA) cycle to generate extra ATP, NADH, and alternative reduced electron carrier flavin adenine dinucleotide (FADH2) in the mitochondria. While NADH produced in the cytoplasm normally needs to be transported into mitochondria via malate-aspartate 
shuttle or donates its electrons via glycerophosphate shuttle for conversion to ATP in electron transport chain (ETC); finally, 32 molecule ATP can be generated from one molecule of glucose (Berg et al., 2002).

Lipids are the major energy reserve, which is stored in the fat body tissue of the fly. Thus, lipid mobilization is critical to fuel energy consuming processes providing the energy supply of Drosophila muscles, for egg production in female and the metabolic maintenance of other tissues (Arrese and Soulages, 2010). The metabolic processes underlying lipid storage and lipolysis are conserved between flies and mammals. Both in flies and mammals, the first step of lipolysis is the breakdown of TAGs by the LD-associated lipase, Brummer, the fly homolog of adipose triglyceride lipase (ATGL) encoded by the gene $\mathrm{bmm}$. This enzyme hydrolyzes TAG to give rise to DAG and FAs (Grönke et al., 2005). In addition, the Drosophila homolog of mammalian hormone sensitive lipase (Hsl), can translocate to LDs in larvae and adult Drosophila, a process that is likely to be necessary for lipolysis under starvation conditions, although loss-of-function $\mathrm{Hsl}$ in adult Drosophila does not prevent normal lipid mobilization during starvation (Grönke et al., 2005; Hehlert, 2016; Kühnlein, 2012). In addition, lipophagy can hydrolyze the lipids of LDs using the autophagy/lysosome system in mammals (Settembre and Ballabio, 2014; Singh et al., 2009). Similarly, autophagy related genes Ykt6 (functional in ER to Golgi mediated vesicle transport) and Rab32 (encoding one Rab GTPases) have been linked to body fat storage and LDs sizes, respectively (Baumbach et al., 2014a; Wang et al., 2012). The breakdown products, DAGs, are possibly transported by lipoprotein lipophorins (assembled from lipoprotein, Apolipophorin) to other tissues such as heart or brain, where they are further processed by lipoprotein lipase to eventually undergo $\beta$-oxidation for AcetylCoA generation (Brankatschk and Eaton, 2010; Lee et al., 2017; Palm et al., 2012).

Concerning the protein, usually it is the last step for mobilization during starvation (Ravussin and Swinburn, 1992). It is not the focus of my project. Therefore, its metabolism is not further discussed in detail.

\subsubsection{Inter-organ regulation of energy homeostasis in Drosophila and human}

Not only individual organ systems of the fly, but also the regulation of energy homeostasis are functionally equivalent to human. This conclusion is based on the finding that inter-organ communication factors are conserved, some of which regulate both local and systemic physiological processes (Droujinine and Perrimon, 
2016). How to bridge the gap between the metabolism at molecular and cellular levels and the different disease phenotypes at organism level, is becoming the focus of network physiology, which investigates how different organs coordinate, synchronize, and integrate their dynamic functions (Ivanov et al., 2016). Here, three aspects of the inter-organ regulation network on energy balance in Drosophila will be introduced: food intake, nutrient storage, and energy mobilization. These three aspects are directly related to the topic of my $\mathrm{PhD}$ study and will be therefore highlighted in the following.

\subsubsection{Inter-organ regulation of food intake}

Regulation of food intake is vital for energy balance of both the fly and human physiological systems. Over the last years, people have validated a large number of conserved food intake regulators in the brain by GWAS (Garlapow et al., 2015), neuronal activity manipulations and behavioral screens (Al-Anzi et al., 2009; Albin et al., 2015) as well as by traditional genetic mutation and neuropeptide overexpression studies (Pool and Scott, 2014). Such studies have identified a number of orexigenic regulators. In mammals, feeding behavior is regulated by the neuropeptide $\underline{Y}$ (NPY) system (Loh et al., 2015; Luquet, 2005). The known Drosophila NPY and receptor homologs are neuropeptide F (NPF) and NPFR (Brown et al., 1999; Feng et al., 2003; Wu et al., 2005), which are required for appetitive memory (Krashes et al., 2009) and their activities are involved in the synthetic sweetener provoked neuronal starvation response pathway in both fly and mice (Wang et al., 2016b). Overexpression of another Drosophila NPY homolog, short neuropeptide F precursor (sNPF), enhances the food intake of flies (Baumbach et al., 2014a; Lee et al., 2004). In addition, certain subsets of serotonergic neurons have also been shown to enhance food intake and to signal a hunger state to the fly (Al-Anzi et al., 2009; Albin et al., 2015). Moreover, trehalose and glucose in the fly hemolymph can directly activate Diuretic hormone 44 (Dh44), a homolog of the mammalian corticotropinreleasing hormone $(\mathrm{CRH})$ which is produced in IPC neurons, and stimulates feeding motivation and gut motility under starvation condition (Dus et al., 2015). Conversely, mutation of the Akh gene, which encodes the adipokinetic hormone Akh, a functional homolog of glucagon in fly, reduces food intake, while overexpressing $A k h$ in fat body tissue promotes feeding (Gáliková et al., 2016). A recent study indicates that four interoceptive neurons expressing AkhR (Akh receptor) in the subesophageal zone (SEZ) directly respond to corpora cardiaca (CC)-secreted Akh also promotes the food intake of the fly (Jourjine et al., 2016). Similarly, some insect-specific neuropeptides or hormones also contribute to food intake regulation. For example, 
activating corazonin $(\mathrm{Crz})$ neurons promote the feeding behavior under starvation condition (Hergarden et al., 2012). Conversely, mutations of the gene CCHa2, which encodes the CCHamide peptide cause reduced food intake (Ren et al., 2015). Apart from neurotransmitters or neuropeptides, gustatory neurons expressing the $G$ protein-coupled receptor (GPCR) receptor Gr43 in adult brain were reported to detect nutritive sugars, which likely get involved in food intake control (Miyamoto et al., 2012).

In addition to food intake promoting factors, there are also neuropeptides representing satiety signals. The mammalian homolog of cholecystokinin (CCK) in fly is Drosulfakinin (DSK), and food intake of the starving flies is significantly increased by down-regulation of DSK expression (Söderberg et al., 2012; Williams et al., 2014). One recent study indicated that the myoinhibitory peptide (MIP) functions as satiety modulator, which is negatively associated with food intake and body weight of the fly (Min et al., 2016).

\subsubsection{Inter-organ regulation of energy storage}

Similar to mammals, the energy storage process is also regulated by insulin signaling in Drosophila. Eight insulin-like peptide (ILP) genes have been identified in fly (Brogiolo et al., 2001; Grönke et al., 2010). Previous studies have shown that different organs communicate with each other to regulate insulin signaling following food intake. Dietary sugar promotes dllp2-3,5 gene expression, and the selective dILP3 release from IPCs of fly larvae (Colombani et al., 2003; Kim and Neufeld, 2015). Moreover, in response to food intake, e.g. amino acids, dILP2 secretion from IPCs is triggered, while food deprivation suppresses the ILPs secretion (Géminard et al., 2009; Park et al., 2014a). IPCs functions like mammalian $\beta$-cells to sense hemolymph glucose levels (Kréneisz et al., 2010; Teleman, 2009). A recent study found that the Drosophila nuclear hormone receptor Hepatocyte nuclear factor 4 (Hnf4), an ortholog of human HNF4A, is essential for insulin peptide secretion in IPCs of adult flies (Barry and Thummel, 2016). Finally, the orexgenic neuropeptide SNPF is likely to promote dllps gene expression through activation of the SNPF receptor, which is encoded by gene sNPFR1 and expressed in IPCs (Lee et al., 2008).

The selective dILP3 release from IPCs is likely to be activated by the secretion of Akh from CC, which is stimulated by hemolymph glucose and trehalose in larvae (Kim and Neufeld, 2015). Moreover, fat body as a peripheral tissue can react to 
increased insulin signaling through sugar uptake, and activation of (Target of rapamycin) TOR signaling (Kim and Neufeld, 2015). Furthermore, insulin signalling promotes fat cell proliferation, and increase fat accumulation via Akt activated shaggy (sgg, glycogen synthase kinase 3) (DiAngelo and Birnbaum, 2009). Also, in response to high fat or high sugar diet, transcription of cytokine unpaired2 (upd2), which is encoded by the gene upd2 and represents the candidate functional homolog of leptin in fly, is induced; its activity finally promotes dILP2,5 secretion (Rajan and Perrimon, 2012). Besides food intake, glucose induces expression and secretion of the nutrient-dependent hormone $\mathrm{CCHamide}-2(\mathrm{CCHa} 2)$ in fat body tissue, which remotely activates dllp-3,5 expression and its secretion through $\mathrm{CCHa} 2$ receptor activity in IPCs (Sano et al., 2015). Fat body might also sense the intracellular sugar level via the transcription factor complex Mondo-Mlx, which directly influences the expression of a signalling ligand, the transforming growth factor $\beta /$ Activin (encoded by the gene dawdle (daw)) (Mattila et al., 2015). Daw plays a crucial role in lipid and carbohydrate metabolism and insulin secretion (Chng et al., 2016). Last but not least, food intake also induces fat body secretion of growth blocking peptides (GBPs), which act also as positive regulators of insulin secretion (Koyama and Mirth, 2016).

\subsubsection{Inter-organ regulation of energy mobilization}

When flies encounter starving condition, energy mobilization requires reduced insulin signalling and active Akh signalling. To reduce nutrient storage and promote the mobilization of molecular fuels, insulin signalling-inhibiting hormones are induced during starvation. They include corpora cardiaca secretion of Limostatin (Lst) (Alfa et al., 2015) and fat body secretion of Eiger (Egr), the Drosophila homolog of mammalian cytokine Tumor Necrosis Factor (TNF) (Agrawal et al., 2016), which are induced by starvation and suppress dILP2 secretion from IPCs (Agrawal et al., 2016; Alfa et al., 2015). Starvation can also induce fat body secretion of another insulin inhibitor, the protein Ecdysone-inducible gene L2 (ImpL-2) (Honegger et al., 2008). ImpL2 acts similar to mammalian IGFBP7, the insulin-like growth factor binding protein 7, which prevents insulin signalling through binding to dILPs (Honegger et al., 2008). More importantly, Akh signalling is indispensable for energy mobilization under energy demanding conditions. Akh is regarded as a functional analogue of glucagon in Drosophila, which is a peptide secreted from a small group of neuroendocrine cells, i.e. the corpora cardiaca cells of flies (Kim and Rulifson, 2004). In vitro biochemical studies indicate that Akh can bind to a GPCR, which is named AkhR in Drosophila (Staubli et al., 2002; Ziegler et al., 1995). In vivo evidence further showed it is expressed in fat body tissues and gustatory neurons in the adult 
subesophageal ganglion region (Bharucha et al., 2008; Park et al., 2002; Staubli et al., 2002). Similar to the function of glucagon secreted from pancreatic islets, Akh regulates hemolymph sugar homeostasis both in larvae and adult flies (Gáliková et al., 2015; Kim and Rulifson, 2004; Lee and Park, 2004). Moreover, Akh/AkhR signalling is critical for lipid mobilization and starvation-induced hyperactivity in starving adult flies (Bharucha et al., 2008; Gáliková et al., 2015; Grönke et al., 2007). A recent study shows that AkhR-positive neurons in the subesophageal zone of the fly brain is required for starvation-induced hyperactivity via octopamine signalling, while insulin signalling exerts a suppressive effect on starvation-induced hyperactivity via the insulin-like receptor ( $\mathrm{InR}$ ) expressed in AkhR positive neurons (Yu et al., 2016).

As for the regulation of Akh secretion, previous studies implicate that starvation increases Akh secretion, as Akh ectopic overexpression in fat body tissue deplete fat storage (Grönke et al., 2007; Lee and Park, 2004). Further, AMP-activated kinase (AMPK) mediated cytosolic $\mathrm{Ca}^{2+}$ is very likely required for the release of Akh upon the transition from high to low trehalose levels, mimicking starvation conditions (Braco et al., 2012). Similarly, AMPK is involved in regulating the calcium levels and glucagon secretion in mammals (Leclerc et al., 2011). Regarding inter-organ communication, studies on another insect species, a locust, indicate that tachykinin peptides and proctolin might promote Akh secretion from corpora cardiaca (Clark et al., 2006; Nässel et al., 1995, 1999). It is unknown, however, whether these peptides also affect Akh secretion in Drosophila.

In contrast with plentiful knowledge on inter-organ regulation of insulin signalling, our knowledge of how fat body tissue or other organs regulate Akh production and secretion is still very limited.

\subsubsection{Cellular regulation of energy homeostasis in Drosophila and human}

Aside from inter-organ communications, a more detailed understanding of how cellular signalling influences energy homeostasis will help us to vertically integrate knowledge of energy regulatory network from cells to tissues (For a simplified view of related key signaling pathways, please see Figure 2).

At the cellular level, insulin regulates fat storage and fat cell activity under feeding condition. Apart from insulin-like peptide genes, other components of the insulin 
pathway are also conserved between fly and human. These components include the insulin-like receptor gene InR (Fernandez-Almonacid and Rosen, 1987), the insulin receptor substrate (IRS) homolog gene chico (Böhni et al., 1999; Goberdhan et al., 1999), the Phosphatidylinositol 3-kinase (PI3K) catalytic subunit gene Dp110 (Leevers et al., 1996), the PI3K adaptor gene p60 (Weinkove et al., 1999), the Akt protein kinase gene Akt, the TOR protein gene Tor (Oldham et al., 2000; Zhang et al., 2000), the S6 kinase gene dS6K (Montagne et al., 1999), the downstream transcription factor forkhead box, sub-group $O$ gene $d F O X O$ as well as its target gene $d 4 E B P$ (encodes eukaryotic translation initiation factor $4 \mathrm{E}$ binding protein) (Jünger et al., 2003; Kramer et al., 2003; Puig et al., 2003). Moreover, Insulin like peptides are confirmed to be essential for carbohydrate homeostasis (Grönke et al., 2010; Rulifson et al., 2002), and insulin signalling can promote fat cell proliferation and fat accumulation (DiAngelo and Birnbaum, 2009). In addition, secreted dILP-2 is known to promote fat body cell growth via the canonical InR/Akt/dFOXO signalling pathway in adult flies (Lee et al., 2008).

Under starvation, Akh binds to AkhR receptor, and facilitates the mobilization of lipids by activating second messenger pathways, including cyclic adenosine monophosphate (cAMP) pathway and calcium pathway, as does the activated mammalian glucagon receptor (Amaya and Nathanson, 2013; Gäde and Auerswald, 2003). In fly, the rapid lipolysis pathway is initiated by Akh/AkhR signalling, which possibly acts through cAMP-PKA (Protein Kinase A) pathway (Grönke et al., 2007). Furthermore, in mammals, $\beta$-adrenergic-cAMP signalling, activated PKA through cAMP-PKA binding, which further phosphorylates lipid droplets (LDs)-localized protein perilipin 1 (PLIN1) (Brasaemle et al., 2000; Tansey et al., 2001). This interaction causes the release of the activator protein ABHD5/CGI-58 to trigger the activity of the ATGL in adipose tissue (Zimmermann et al., 2004) and to initiate the subsequent lipid catabolism by recruiting the Hsl lipase (Souza et al., 2002). Likewise, Drosophila PLIN1 resides at the lipid droplet membrane (Beller et al., 2010). PLIN1 is essential for Akh/AkhR regulated lipolysis by modulating the access of the Drosophila ATGL homolog Brummer (BMM) to the lipid droplet surface (Beller et al., 2010). Another study indicates that recruitment of Drosophila Hsl to lipid droplet also requires PLIN1 under starvation conditions (Bi et al., 2012). Moreover, Akh/AkhR likely regulates lipolysis at transcription level via the cAMP-responsive transcription factor Cyclic-AMP response element binding protein B (dCREB2). Thus overexpression of dominant-negative form of dCREB2 in fat body tissue not only increased lipid content (lijima et al., 2009), but also repressed the expression of 
lipase gene $b m m$ and increased the expression of the lipid synthesis gene mdy (Baumbach, 2014). In fact, to meet energy demands, basally and partially induced lipolysis are also independently carried out by BMM (Grönke et al., 2007). Bmm's expression is up-regulated by transcription factor dFOXO under starvation conditions in flies (Wang et al., 2011). Nevertheless, it remains unclear whether other upstream factors also regulate BMM's expression.

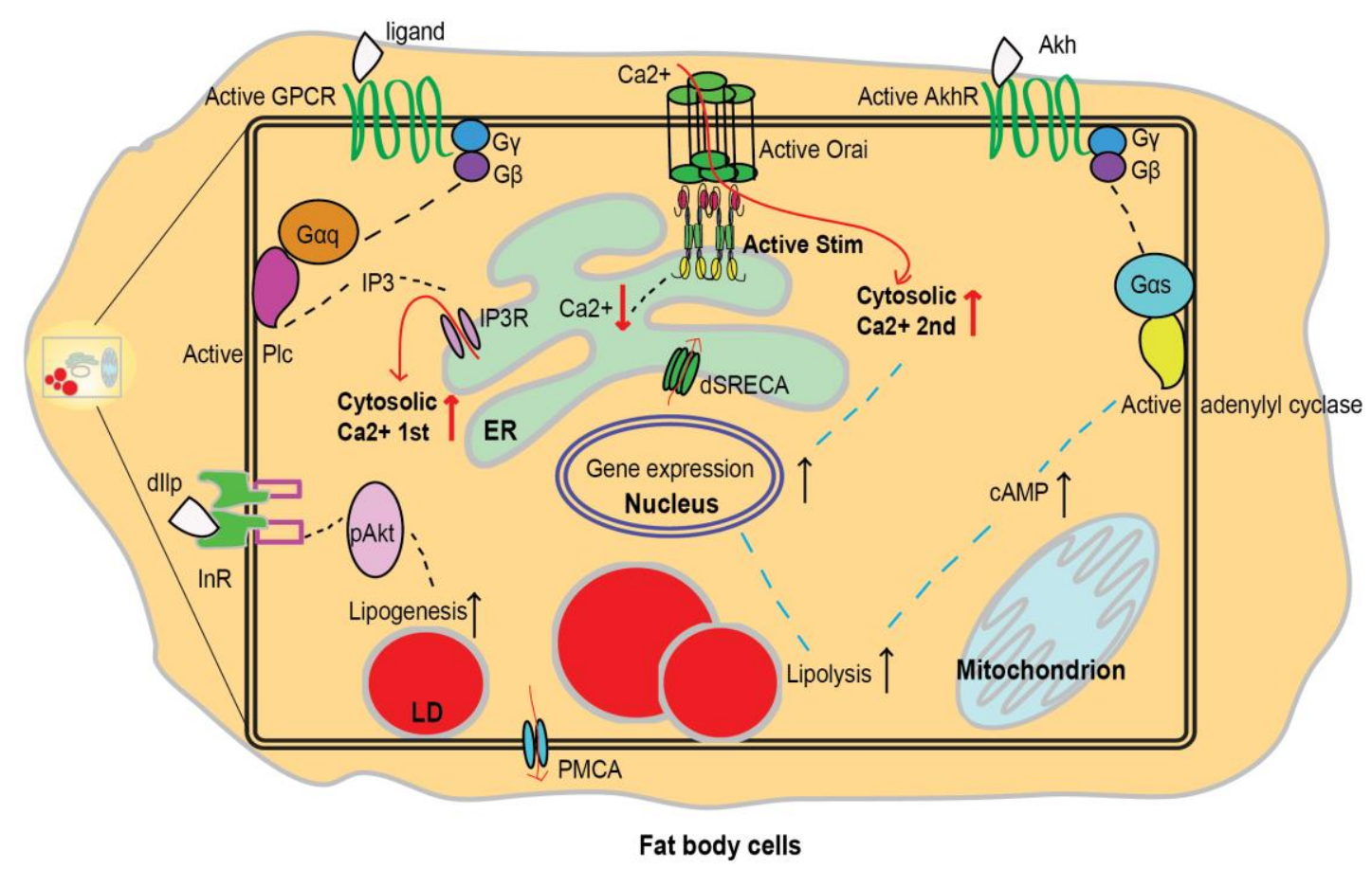

Figure 2. Scheme of fat storage control by the Store-Operated Calcium Entry (SOCE) in response to GPCR/Gaq/Plc pathway, as well as by Akh/AkhR/Gas/adenylyl cyclase/cAMP, and insulin signalling pathways

In Drosophila, first, lipogenesis can be promoted by the insulin-like peptide (dILP)/Insulin receptor (InR)/Akt signalling pathway. On one hand, lipolysis can be directly promoted by the adipokinetic hormone (Akh)/AkhR/Gas/adenylyl cyclase/cAMP signalling pathway. On the other hand, ligand binds to and activates G-protein coupled receptor (GPCR), which further activate $\mathrm{G}$ protein subunit Gaq to activate phospholipase C (PIc). Active PIc generate inositol 1,4,5-trisphosphate (IP3), which activate the IP3 receptor (IP3R) located on the Endoplasmic Reticulum (ER) membrane. IP3Rs release the $\mathrm{Ca}^{2+}$ store of ER and cause first elevation of cytosolic $\mathrm{Ca}^{2+}$ level. The depletion of $\mathrm{ER} \mathrm{Ca}^{2+}$ is sensed by Stim protein, which further translocate to ER-plasma membrane junction areas. Stim proteins further facilitate the activation of Orai proteins, which allow the influx of extracellular $\mathrm{Ca}^{2+}$ into cytoplasm. Thus the $2^{\text {nd }}$ elevation of cytosolic $\mathrm{Ca}^{2+}$ level ensues, which regulates the possible expression of lipolysis genes. The sarco(endo)plasmic reticulum calcium ATPase (dSERCA in Drosophila) and plasma membrane calcium ATPase (PMCA) gradually pumps cytosolic $\mathrm{Ca}^{2+}$ into ERs or into extracellular space. The figure is done with Adobe llustrator and Pathvisio (https://www.pathvisio.org/) based on figures in (Baumbach et al., 2014a), (Deng et al., 2015) and (Gäde and Auerswald, 2003). 
Apart from cAMP, cytosolic $\mathrm{Ca}^{2+}$ level also responds to Akh/AkhR signalling in fat body cells (Vroemen et al., 1995). Similar to cAMP, cells also transduce diverse environmental signals into intracellular $\mathrm{Ca}^{2+}$ concentration changes, which directly alter numerous proteins' conformations and their interactions by binding or dissociating from $\mathrm{Ca}^{2+}$. Factors involved in the regulation of cellular $\mathrm{Ca}^{2+}$ levels include kinases, phosphatases, the calcium binding protein calmodulin, as well as transcription factors (Clapham, 2007). For example, a continuous high $\mathrm{Ca}^{2+}$ level, which results from store-operated calcium entry (SOCE) activity, is essential for Nuclear factor of activated T cells (NFAT)-mediated transcription in mammalian cells (Dolmetsch et al., 1997). This mechanism is used to establish the NFAT-based cytosolic calcium reporter system- calcium-dependent nuclear import of Lex A (CaLexA) in Drosophila (Masuyama et al., 2012).

As a matter of fact, $\mathrm{Ca}^{2+}$ is a signalling hub for the regulation of a plethora of cellular processes over a wide temporal spectrum. These processes include exocytosis, contraction of muscle cells, metabolism, transcription, fertilization and proliferation of cells (Berridge et al., 2000, 2003). To regulate these diverse processes and avoid possible precipitating of $\mathrm{Ca}^{2+}$ in the presence of phosphate, which is another universal component in signal transduction processes, cytosolic $\mathrm{Ca}^{2+}$ is finely tuned by the SOCE system (Clapham, 2007; Soboloff et al., 2012). Its core components are conserved between fly and man, including the stromal interaction molecule (STIM) (Baumbach et al., 2014a; Roos et al., 2005). The SOCE action involves two inter-connected steps. The first step is the binding of extracellular hormones to plasma membrane protein such as GPCR or the initial calcium increase due to plasma membrane calcium channels in muscle cells. If the GPCR receptor becomes activated, it triggers the separation of the $G \alpha$ subunit protein from $G \beta \gamma$ subunits protein by catalyzing the replacement of GDP by GTP at the Ga subunit. The released $G \alpha$ subunit in turn stimulates phospholipase $C$ (PIc $\beta)$, which cleaves Phosphatidylinositol 4,5-bisphosphate (PIP2) into DAG and inositol 1,4,5trisphosphate (IP3), which also serves as the second messengers; diffusing IP3 further stimulates a first $\mathrm{Ca}^{2+}$ release from endoplasmic reticulum (ER) stores by activating its calcium channel-receptor IP3R (or via $\mathrm{Ca}^{2+}$-sensitive ryanodine receptors (RyR)) (Berridge et al., 2003; Clapham, 2007). Then, the second step ensues. Another kind of ER membrane proteins and the STIM1, which was encoded by Stim gene in Drosophila S2 cells (Roos et al., 2005), senses the ER calcium depletion through an ER luminal calcium binding domain, the EF-hand domain. Next, oligomerized STIM1 proteins migrate into ER-plasma membrane junctions and 
translocate their cytoplasmic tails to cell plasma membrane, where 8 extended STIM1 cytoplasmic domains interact with 4 ORAI1 (Calcium release-activated calcium channel protein 1) proteins to form active $\mathrm{Ca}^{2+}$ release-activated $\mathrm{Ca}^{2+}$ channels (CRACs) (Feske et al., 2006; Vig et al., 2006; Zhang et al., 2006), which ultimately facilitate the second extracellular calcium entry by opening CRACs (Liou et al., 2005; Zhang et al., 2005). SOCE enables cells to increase the average cytosolic $\mathrm{Ca}^{2+}$ concentration from around $100 \mathrm{nM}$ to around $1 \mu \mathrm{M}$ (Bootman, 2012; Clapham, 2007). Following SOCE activity, cytosolic $\mathrm{Ca}^{2+}$ is maintained at resting level by pumping the extra cytosolic $\mathrm{Ca}^{2+}$ back into the $\mathrm{ER}$, a process that involves the sarco(endo)plasmic reticulum calcium ATPase (SERCA) (reviewed in Clapham, 2007). Alternatively, $\mathrm{Ca}^{2+}$ is extruded from the cell by the $\underline{\mathrm{Na}^{+}} / \underline{\mathrm{C}} \mathrm{a}^{2+}$ exchanger (NCX) or in response to the plasma membrane calcium ATPase (PMCA) (Brini et al., 2013; Clapham, 2007; Raffaello et al., 2016). Moreover, cytosolic $\mathrm{Ca}^{2+}$ dynamics can interact with mitochondrial $\mathrm{Ca}^{2+}$ uptake. It has been shown that high $\mathrm{Ca}^{2+}$ level promotes $\mathrm{Ca}^{2+}$ uptake into mitochondria, which not only adjusts cytosolic $\mathrm{Ca}^{2+}$ buffering but also affects mitochondrial effectors (Raffaello et al., 2016; Rizzuto et al., 2012). Finally, lysosomes likely contribute to the regulation of $\mathrm{Ca}^{2+}$ homeostasis by serving as $\mathrm{Ca}^{2+}$ stores (Raffaello et al., 2016).

In fact, SOCE trigger the translocation of NFAT into nucleus in immune cells, which is essential for the expression of a number of genes (Niemeyer, 2016). It has been well defined that loss of function mutations of ORAI1 and STIM1 in human cause diseases such as severe immunodeficiency (Lacruz and Feske, 2015). Recently, several recent studies in Drosophila demonstrate the negative link between cytosolic $\mathrm{Ca}^{2+}$ level and adult body fat storage. Hypomorphic mutations of IP3R cause obese flies (Subramanian et al., 2013), and inhibiting dSERCA activity results in lipid droplet depletion in fat body tissues (Bi et al., 2014a). Knockdown of the key player of the SOCE system, such as the $G$ protein subunit ( $G \alpha, G \gamma)$ and the Plc $\beta$ gene, promote body fat storage in the adult flies (Baumbach et al., 2014a; Bi et al., 2014a; Subramanian et al., 2013). Likewise, in mammals, calcium signalling is also involved in organelle dysfunctions such as ER, and mitochondria of various metabolic tissues (Arruda and Hotamisligil, 2015; Rizzuto et al., 2012). Recent human GWAS studies identified one quantitative genetic locus (Single Nucleotide Polymorphism, SNP) localized in the calcium signalling gene IP3R, which is significantly associated with body fat distribution (Shungin et al., 2015); one SNP located at STIM2 is significantly associated with obesity-related traits (Rouillard et al., 2016) . Moreover, a most recent study reported that SOCE plays a crucial role for lipolysis induction and 
transcriptional regulation of lipid metabolism genes in mouse liver, heart, and skeletal cells as well as isolated human fibroblasts cells (Maus et al., 2017). Collectively, these studies underline a central role played by cytosolic $\mathrm{Ca}^{2+}$ levels for the lipid metabolism of different organs.

However, the studies mentioned above on the impact of SOCE on lipid metabolism have inherent methodological limitations. Most of published studies were based on the ubiquitous impairing of SOCE key components in the organism, which cause lethality or elicit compensatory effects. For flies, only hypomorphic dIP3R mutants are viable (Subramanian et al., 2013). Furthermore, ubiquitous ORAl1 knock-in mice are leathal (Maus et al., 2017), similarly, both ubiquitous loss-of-function mutation of STIM1/2 in mice and ubiquitous loss-of-function mutation of Stim, the homologue gene of STIM1/2 in Drosophila provokes lethality (Maus et al., 2017; Pathak et al., 2017). Therefore, the impact of reduced or lacking SOCE activity in a specific organ and the long-term influences of SOCE dysfunction on fat body tissue is unknown. Meanwhile, a previous study found that flies become obese in response to an acute reduction of Stim activity specifically in fat storage tissue of flies (Baumbach et al., 2014a). However, in this study Stim RNAi was induced for a 6 day period in a temperature-dependent manner at $29^{\circ} \mathrm{C}$, a temperature which also entails temperature-associated stress on fly energy homeostasis (Sayeed and Benzer, 1996). The potential interactions between temperature-associated stress and genetic background may also generate temperature dependent cis or trans-regulatory effects on gene expression (Chen et al., 2015), which may undermine our understanding of how SOCE dysfunction affects energy homeostasis.

In order to overcome the experimental limitations that might be associated with the six days lasting thermal stress conditions, comparatively short-term Stim knockdown period in fat storage tissues was performed and shown to causes a long-term effect on fat accumulation, resulting in obese flies even 30 days after they passed the 34 hours thermal treatment (Baumbach, 2014; Borcherding, 2014). However, the exact phenotypes were not completely characterized, and their underlying mechanisms were not systematically investigated. To further the causal link between fat storage and short-term Stim knockdown, I follow the same strategy and reduced the fat storage-specific Stim RNAi expression from long-term (6 days) to transient (38 hours). I focused on the characterization of the obesity phenotype in response to the transient temperature-shift Stim RNAi expression and the possible mechanisms associated with it. 
It is still obscure how the SOCE in response to upstream signalling affect fat storage. Further, it is unclear that how transient expression of Stim in the fat storage organ may lead to pathogenesis of obesity in vivo. In addition, how SOCE ties into the multiple-level, horizontal and vertical interactions of the energy regulatory network components is also unknown. Thus, several open questions remain to close the gap between SOCE dysfunction and the resulting limitations of the energy regulatory network to defend obesity. Drosophila fat storage tissue is functionally homologous to mammal white adipose tissue, and understanding the regulatory roles of SOCE in fat storage tissue will inspire our research on human obesity and its related diseases. I took advantage of the powerful model system Drosophila to approach the following questions within the frame of my PhD thesis:

(1) Which upstream regulator genes of Stim control energy homeostasis in response to Akh/AkhR signalling?

(2) Which kinds of obese phenotypes develop after transient Stim RNAi in fat storage tissue?

(3) How does transient Stim RNAi mis-regulates other signalling pathways in adipose cells as well as inter-organ communication, and finally causes dysfunction of systemic energy homeostasis? 


\section{Materials and methods}

\subsection{Fly stocks, husbandry and genetics}

\subsubsection{Fly stocks}

Details of fly stocks used in this study are described in of fly stocks.

Table 1 Information of fly stocks

\begin{tabular}{|c|c|c|c|}
\hline Short Name & $\begin{array}{c}\text { Stock } \\
\text { Number }\end{array}$ & Genotype & $\begin{array}{l}\text { Reference/ } \\
\text { Source }\end{array}$ \\
\hline w-control & RKF1084 & $w[1118] ;+/+;+/+$ & VDRC60000 \\
\hline$t s-F B-G a / 4$ & RKF805 & 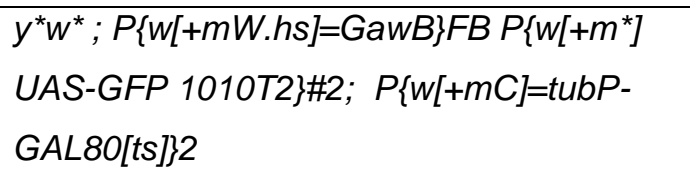 & $\begin{array}{c}\text { (Beller et al., } \\
\text { 2010) }\end{array}$ \\
\hline FB-Gal4 & RKF125 & $w^{*} ; P\{w[+m W \cdot h s]=G a w B\} F B+S N S ;+/+$ & $\begin{array}{c}\text { (Grönke et al., } \\
\text { 2003) }\end{array}$ \\
\hline Akh-Gal4 & RKF694 & $\begin{array}{l}w^{*} ; \text { akhp-GAL4, UAS-mCD8 GFP ; akhp- } \\
\text { GAL4/ SM5a-TM6 Tb }\end{array}$ & $\begin{array}{l}\text { (Kim and } \\
\text { Rulifson, } \\
\text { 2004) }\end{array}$ \\
\hline da-GS & MGF1663 & $w[1118] ;$ da-GS; +/+ & $\begin{array}{c}\text { (Tricoire et al., } \\
\text { 2009) }\end{array}$ \\
\hline Stim RNAi1 & RKF1112 & w[1118]; UAS dSTIM[RNAi] / CyO float; +/+ & $\begin{array}{r}\text { (Eid et al., } \\
2008)\end{array}$ \\
\hline Stim RNAi2 & RKF1113 & $w[1118] ;+/+;$ UAS dSTIM[RNAi] / TM3, Sb* & $\begin{array}{r}\text { (Eid et al., } \\
2008)\end{array}$ \\
\hline Stim RNAi3 & RKF1178 & $w[1118] ;+/+; P\{G D 16187\} v 47073$ & VDRC 47073 \\
\hline Plc21C RNAi1 & YXF1415 & $w[1118] ; P\{G D 11359\} v 26558 ;+/+$ & VDRC26558 \\
\hline Plc21C RNAi2 & YXF1416 & $\begin{array}{l}y[1] s c\left[^{\star}\right] v[1] ;+/+; P\{y[+t 7.7] \\
v[+t 1.8]=T R i P . H M S 00600\} \text { attP2 }\end{array}$ & BDSC33719 \\
\hline Gr1 RNAi1 & YXF1413 & $w[1118] ; P\{G D 13720\} v 28844 / C y O ;+/+$ & VDRC28844 \\
\hline Gr1 RNAi2 & YXF1414 & $\begin{array}{l}y[1] v[1] ;+/+; P\{y[+t 7.7] \\
v[+t 1.8]=T R i P . J F 01954\} a t t P 2\end{array}$ & BDSC25934 \\
\hline Gaq RNAi1 & YXF1411 & $w[1118] ;+/+; P\{G D 8609\} v 19088$ & VDRC19088 \\
\hline Gaq RNAi2 & YXF1412 & $\begin{array}{l}y[1] v[1] ;+/+; P\{y[+t 7.7] \\
v[+t 1.8]=T R i P . J F 02464\} a t t P 2\end{array}$ & BDSC33765 \\
\hline AkhR RNAi1 & YXF1410 & $w[1118] ;+/+; P\{G D 586\} v 9546 / T M 3 S b$ & VDRC9546 \\
\hline AkhR RNAi2 & & $\begin{array}{l}y[1] v[1] ; P\{y[+t 7.7] \\
v[+t 1.8]=T R i P . H M C 03228\} a t t P 40 ;+/+\end{array}$ & BDSC51710 \\
\hline$A k h R N A i$ & RKF1737 & $\begin{array}{l}y[1] s c\left[^{*}\right] v[1] ;+++; P\{y[+t 7.7] \\
v[+t 1.8]=T R i P . H M S 00477\} a t t P 2\end{array}$ & BDSC34960 \\
\hline
\end{tabular}




\begin{tabular}{|c|c|c|c|}
\hline Akh gof & RKF656 & $y[1] w[*] ; P\{w[+m C]=U A S-A k h . L\} 2 ;+/+$ & $\begin{array}{c}\text { (Lee and Park, } \\
\text { 2004) } \\
\text { BDSC27343 }\end{array}$ \\
\hline$A k h R^{1}$ & RKF639 & $y^{*}$ float $w^{*} ; A k h R[1] /$ CyO float; $+/+$ & $\begin{array}{c}\text { (Grönke et al., } \\
\text { 2007) }\end{array}$ \\
\hline CaLexA reporter & RKF1324 & $\begin{array}{l}++; \text { LexAop-CD8-GFP-2A-CD8-GFP / } \\
\text { LexAop-CD8-GFP-2A-CD8-GFP; UAS- } \\
\text { mLexA-VP-NFAT C del (4-2), LexAop-CD2- } \\
\text { GFP /TM6B, Tb* }\end{array}$ & $\begin{array}{c}\text { (Masuyama et } \\
\text { al., 2012) }\end{array}$ \\
\hline $\begin{array}{l}\text { FB-Gal4; } \\
\text { AkhR RNAi }\end{array}$ & YXF1417 & $\begin{array}{l}w^{*} ; P\{w[+m W . h s]=G a w B\} F B+S N S / C y O \\
\text { float.; AkhR RNAi/TM3,Ser }{ }^{*}\end{array}$ & $\begin{array}{r}\text { (Baumbach et } \\
\text { al., 2014b) }\end{array}$ \\
\hline $\begin{array}{l}\text { FB-Gal4; } \\
\text { Goq RNAi2 }\end{array}$ & YXF1418 & 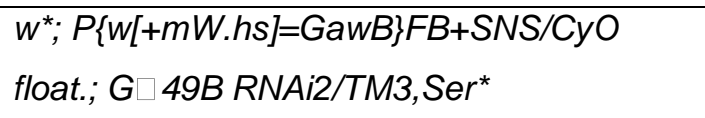 & $\begin{array}{r}\text { (Baumbach et } \\
\text { al., 2014b) }\end{array}$ \\
\hline $\begin{array}{l}\text { FB-Gal4; } \\
\text { Gr1 RNAi2 }\end{array}$ & YXF1419 & $\begin{array}{l}w^{*} ; P\{w[+m W . h s]=G a w B\} F B+S N S / C y O \\
\text { float.; Gy1 RNAi2/TM3,Ser }\end{array}$ & $\begin{array}{r}\text { (Baumbach et } \\
\text { al., 2014b) }\end{array}$ \\
\hline $\begin{array}{l}\text { FB-Gal4; } \\
\text { Plc21C RNAi2 }\end{array}$ & YXF1420 & $\begin{array}{l}w^{*} ; P\{w[+m W . h s]=G a w B\} F B+S N S / C y O \\
\text { float.; Plc21C RNAi2/TM3,Ser }\end{array}$ & $\begin{array}{r}\text { (Baumbach et } \\
\text { al., 2014b) }\end{array}$ \\
\hline $\begin{array}{l}\text { FB-Gal4; } \\
\text { Stim RNAi2 }\end{array}$ & JBF1425 & $\begin{array}{l}w^{*} ; P\{w[+m W . h s]=G a w B\} F B+S N S / C y O \\
\text { float.; Stim RNAi2/TM3,Ser }{ }^{*}\end{array}$ & $\begin{array}{r}\text { (Baumbach et } \\
\text { al., 2014b) }\end{array}$ \\
\hline$A k h^{A}$ & MGF1639 & $\begin{array}{l}\text { w[1118]; akhp-GAL4, UAS-mCD8 GFP } \\
\text { /CyO; Akh[A]/ TM3 Ser* }\end{array}$ & $\begin{array}{l}\text { (Gáliková et al., } \\
\text { 2015) }\end{array}$ \\
\hline dllp2-3,5 mutant & RKF1661 & $\begin{array}{l}W^{*} ;+/+; \text { dilp2,3,5/TM3 } \text { Ser }^{*} P\{w[+] A c t \\
\text { GFP }\} \text { float. }\end{array}$ & $\begin{array}{c}\text { (Grönke et al., } \\
\text { 2010) }\end{array}$ \\
\hline dFOXO[delta94] & RKF1301 & $w[D a h] ;+/+;$ foxo[delta94]/TM3 Tb[-] Ubx[-] & $\begin{array}{c}\text { (Slack et al., } \\
\text { 2011) }\end{array}$ \\
\hline dilp6[41] & RKF1560 & dilp6[41] $y^{*} ;+/+;+/+$ & $\begin{array}{c}\text { (Grönke et al., } \\
2010)\end{array}$ \\
\hline daGS; $A k h^{A}$ & YXF1765 & $w^{*} ;$ daGS/CyO; AkhA/TM3Ser & This study \\
\hline Stim RNAi1; $A k h^{A}$ & YXF1766 & w $^{*}$; StimRNAi/CyO; AkhA/TM3Ser & This study \\
\hline $\begin{array}{l}\text { daGS; } \\
\text { dllp2-3,5 mutant }\end{array}$ & YXF1757 & $\begin{array}{l}w^{*} ; \text { daGS/CyO; dllp2-3,5 triple } \\
\text { mutant/TM3Ser }\end{array}$ & This study \\
\hline $\begin{array}{l}\text { Stim RNAi1; } \\
\text { dllp2,3,5 mutant }\end{array}$ & YXF1758 & $w^{*}$, StimRNAi/CyO; dllp2,3,5 triple mutant & This study \\
\hline $\begin{array}{l}\text { daGS; } \\
\text { dFOXO[delta94] }\end{array}$ & YXF1759 & $w^{*}$, daGS/CyO, foxo[delta94]/TM3Ser & This study \\
\hline $\begin{array}{l}\text { Stim RNAi1; } \\
\text { dFOXO[delta94] }\end{array}$ & YXF1760 & w*; StimRNAi/CyO; foxo[delta94]/TM3Ser & This study \\
\hline daGS; dilp6[41] & YXF1761 & dllp6[delta41]/dllp6[delta41]; daGS/CyO; +/+ & This study \\
\hline Stim RNAi1; & YXF1762 & dllp6[delta41]/dllp6[delta41]; StimRNAi/CyO; & This study \\
\hline
\end{tabular}




\begin{tabular}{|c|c|c|c|}
\hline dilp6[41] & & $+/+$ & \\
\hline balancer line 1 & RKF891 & $\begin{array}{l}w^{*} ; w g[S p-1] / C y O P\{r y f t z-l a c Z\} ; D^{*} / T M 3 \\
S b, P\{w[+] \cup b x-l a c Z\}\end{array}$ & A. Herzig \\
\hline balancer line 2 & RKF1365 & $w\left[^{\star}\right] ; \operatorname{Kr}[l f-1] / C y O ; D[1] / T M 3, \operatorname{Ser}[1]$ & BDSC7198 \\
\hline $\begin{array}{l}\text { Stim }-R N A i 1 \text { resistan } \\
\text { cDNA RA modified } \\
\text { transgene }(R m)\end{array}$ & YXF1569 & $\begin{array}{l}y[1] \text { M\{vas-int.Dm\}ZH-2A } w^{*} ;+++; \\
\text { pUASTattb-Stim cDNA RA modified Stim } \\
\text { RNAi1 resistant construct(RM) 3rd } \\
\text { chromosome Transgenic line 1/TM3-Sb }\end{array}$ & This study \\
\hline $\begin{array}{l}\text { Stim } c D N A R A \\
\text { transgene }\end{array}$ & YXF1574 & $\begin{array}{l}y[1] \text { M }\left\{\text { vas-int.Dm\}ZH-2A } w^{*} ;+++;\right. \\
\text { pUASTattb-Stim cDNA RA construct(RA) } \\
\text { 3rd chromosome Transgenic line 1/TM3-Sb }\end{array}$ & This study \\
\hline Stim RNAi1\&Rm & & $\begin{array}{l}w^{*} ; \text { StimRNAi1/ CyO; Stim-RNAi1 resistant } \\
\text { cDNA RA modified/ TM3Ser }\end{array}$ & This study \\
\hline $\begin{array}{l}\text { Stim RNAi1; } \\
\text { Stim-cDNA RA } \\
\text { (Stim RNAi1\&RA) }\end{array}$ & & $\begin{array}{l}\text { W*; StimRNAi1/ CyO; Stim cDNA RA/ } \\
\text { TM3Ser }\end{array}$ & This study \\
\hline FBI-26-GS-Gal4 & RKF1045 & $+; P\{$ Switch1\}FBI-26; UAS-GFP & $\begin{array}{c}\text { (Suh et al., } \\
\text { 2007) }\end{array}$ \\
\hline TI-GS-Gal4 & RKF1664 & wDah; TIGS-GS; +/+ & $\begin{array}{c}\text { (Alic et al., } \\
\text { 2014; } \\
\text { Poirier et } \\
\text { al., 2008) }\end{array}$ \\
\hline GSG8708-Gal4 & & $\begin{array}{l}W^{*} ; \\
P\{w[+m W . h s]=S w i t c h 2\} C G 2765[G S G 8708] \\
\text { CyO; +/+ }\end{array}$ & BDSC41013 \\
\hline Elav-GS-Gal4 & RKF903 & $w[1118] ;+/+;$ P elav-Switch.O\}GS & $\begin{array}{c}\text { (Osterwalder et } \\
\text { al., 2001) }\end{array}$ \\
\hline promE800-GS-Gal4 & YXF1690 & w1118; +/+ ; promE(800)GSGFP/TM3 & $\begin{array}{c}\text { (Chatterjee et } \\
\text { al., 2014) }\end{array}$ \\
\hline
\end{tabular}

\subsubsection{Fly husbandry}

Flies were raised on "Göttingen food" (Gö-food; $20 \mathrm{~L} \mathrm{H}_{2} \mathrm{O}, 125 \mathrm{~g}$ agar, $360 \mathrm{~g}$ dry yeast, $200 \mathrm{~g}$ soy flour, $440 \mathrm{~g}$ treacle, $1.6 \mathrm{~kg}$ cornmeal, $1.6 \mathrm{~kg}$ malt, $125 \mathrm{~mL}$ propionic acid and $30 \mathrm{~g}$ nipagine) distributed into midsize vials (20 mL, "food vials"; PS-Dosen, 68 mL, 36/83mm, Greiner Bio-One, Kremsmünster, Germany, Cat. \#: 217101) into which a filter paper (Filterpaper folded $\varnothing 7 \mathrm{~cm}$; Macherey-Nagel, Düren, Germany, Cat. \#: 531007) was inserted. A mite sponge plug (Ø36 mm, Mite proof plug, K-TK, Retzstadt, Germany) was put into vial to stop fly from escaping. Flies were kept at 
either $25^{\circ} \mathrm{C}$ or $18^{\circ} \mathrm{C}$. For experiments, 200 embryos were transferred into a food vials and, after eclosion, 100 adult male or female flies were collected and kept in fresh food vials. Alternatively, in some of the feeding experiments, flies were kept on food vials containing a mixture of yeast (Brecht, Eggenstein, Germany) (=Y.; $2 \%$ or $12 \%$ $\mathrm{w} / \mathrm{w}$ ) and Sucrose (= S.; $10 \%$ or $40 \% \mathrm{w} / \mathrm{w}$ ) in $1 \%$ agar (Probio $\mathrm{GmbH}$, Eggenstein, Germany) in water; the food mixture was heated to dissolve the agar and cooled down to room temperature to solidify the agar again.

\subsubsection{Fly genetics for non-conditional RNAi expression and CaLexA expression}

Fly stocks containing FB-Gal4 fat body driver or UAS-RNAi transgene were balanced by crossing with double balancer lines. Then balanced FB-Gal4 fat body driver flies and balanced UAS-RNAi transgene flies were crossed together to establish the fly stocks containing both FB-Gal4 fat body driver and UAS-RNAi transgene (FBGal4/UAS RNAi lines). Afterwards, the virgin females of FB-Gal4/UAS RNAi lines were crossed with the adult males of CaLexA transgenic line, respectively. Their offspring were kept for development until 6-7 days after eclosion at $18^{\circ} \mathrm{C}$ to reduce FB-Gal4-driven UAS-RNAi and CaLexA expression to minimum level. Due to the existence of balancer in FB-Gal4/UAS RNAi lines, the offspring consists of two kinds of flies: FB-Gal4/UAS RNAi/CaLexA flies (RNAi On) and FB-Gal4/balancer /CaLexA flies (RNAi Off). RNAi On and RNAi Off flies were collected and were further transferred to $25^{\circ} \mathrm{C}$ for 5 days RNAi expression and CaLexA system activation before the CaLexA GFP reporter imaging.

\subsubsection{Fly genetics for temperature-shift induced RNAi expression}

Transient temperature-shift RNAi experiments were performed as described by (Baumbach et al., 2014a) with modifications as outlined below. Briefly, virgin female flies containing the tissue-specific, temperature-sensitive Gal4 fat body driver (ts-FBGal4) were mated with males of the indicated UAS-RNAi transgenic lines. In parallel, wild type control (w[1118]) virgins were crossed with corresponding UAS-RNAi transgenic lines. After hatching, the offspring flies were kept at $18^{\circ} \mathrm{C}$. On day six after hatching, the flies containing both the RNAi and the Gal4 driver transgene were exposed to $29^{\circ} \mathrm{C}$ to induce and maintain RNAi expression for either 6 days temperature shift induced RNAi expression ( 6 days RNAi On) or 38 hours Transient temperature-shift Induced RNAi expression (TtI On). Corresponding flies, which does not express RNAis at $18^{\circ} \mathrm{C}$ are referred to as 6 days RNAi Off, or "Ttl Off". 
In addition to the Ttl Off control flies, flies only containing a UAS-RNAi transgenic construct (that was not sensitive to the temperature-shift) were also exposed to $29^{\circ} \mathrm{C}$ for 38 hours serving as an additional control referred to as "Ttl On negative control". If such flies were not exposed to the temperature shift, they are referred to as "Ttl Off negative control". In order to specify the RNAi that are inducible or not expressed (controls) from the transgene, the abbreviation of the gene name was added in front. This means that in the context of flies containing a Stim RNAi transgene, and dependent on their treatment, four types of experimental and control flies were generated, i.e. Stim-TtI On and Stim-Off as well as Stim-Ttl On negative control and Stim-Ttl Off negative control were used.

\subsubsection{Fly genetics for transient drug-feeding induced RNAi expression}

In order to induce the RNAi expression from a transgene in response to a drug, virgin females containing e.g. the drug-inducible driver daGS (Tricoire et al., 2009) (or other drug-inducible drivers), were used in combination with a UAS-dependent RNAi transgene provided through the males. In parallel, wild type control (white eye) virgin female or male flies were used in combination with UAS-Stim RNAi1 in male or daGS driver in virgin females respectively (w-control x Stim RNAi1 and w-control x daGS). These flies were kept at $25^{\circ} \mathrm{C}$ before drug feeding-induced RNAi expression. The drug (mifepristone, RU486; Sigma-Aldrich, Cat. \#: M8046-500MG) was applied at $200 \mu \mathrm{M}$ concentration added to the food, and four days old flies containing both transgenes were fed with the drug-containing food for four days. After drug feeding, flies were transferred to normal food and kept for the time period reported in the result section. Experiments with flies containing both the daGS and the UAS transgene are referred to as Transient drug-feeding Inducible flies (Tdl) flies, which were fed with the drug as Tdl On, while flies which were continiously fed with normal food lacking the drug as Tdl Off. The Stim-Tdl experimental system used in the present study is therefore based on Stim-Tdl On and Stim-Tdl Off flies. Experiments with flies containing only the UAS transgene (w-control x Stim RNAi1) or only the daGS (w-control $\mathrm{x}$ daGS) are referred to as Tdl negative control 1 and 2 , respectively. Flies, which were fed with the drug as TdI On negative control 1 and 2, while flies, which were continuously fed with normal food lacking the drug as Tdl Off negative control 1 and 2 (see supplementary Figure 4G).

\subsubsection{Stim cDNA or RNAi resistant cDNA construct transformation}

In order to test whether Stim mRNA is the primary or exclusive target of Stim RNAi, I generated a UAS-Stim RNAi1 resistant transgene (collaboration with Dr. J. 
Baumbach) by modifying the wild type Stim cDNA (see 2.2.1.5). Plasmid containing the UAS-Stim cDNA RA (UAS-Stim RA) construct or UAS-Stim RNAi1 resistant cDNA RA modified (UAS-Stim Rm) construct were injected into embryos ( $y w$, BDSC24749) to integrate them into the $3^{\text {rd }}$ chromosome, region 86F8, which employed the PhiC31 integrase system to mediate site-specific insertion (Bischof et al., 2007; Groth et al., 2004; Venken and Bellen, 2007) (BestGen Inc., http://www.thebestgene.com). The proper insertion was confirmed by PCR analysis (BestGen Inc.,).

\subsubsection{Genetic crosses for combining transgenes or mutations together}

In order to test the effects of Stim-Tdl in the context of different mutants or transgene-dependent gene over-expression studies in genetic interaction experiments, the daGS driver was combined with different mutants, with UAS-Stim RNAi1 or with other UAS transgenes (UAS-Stim RNAi resistant cDNA RA modified (UAS-Stim Rm) or UAS-Stim cDNA RA (UAS-Stim RA)). Genetic crosses were designed based on principles described in "Fly pushing" (Greenspan, 2004). Since both, the daGS transgene and UAS-Stim RNAi1 were localized at the $2^{\text {nd }}$ chromosome, daGS driver containing males were balanced with virgin females containing the double balancer RKF1365 to generate F1 male offspring, which carry second chromosome marker ( $\mathrm{Kr}[\mathrm{lf}-1]$, small eye) and the $3^{\text {rd }}$ chromosome balancer TM3, Ser[1]. On the other hand, flies bearing the UAS-Stim cDNA RA wild type transgene, the UAS-Stim-RNAi1 resistant cDNA RA transgene or other mutants, which are located on the $3^{\text {rd }}$ chromosome, were balanced with the RKF891 double balancer. In this way, F1 virgin females were generated, which carry a $2^{\text {nd }}$ chromosome balancer (CyO $\mathrm{P}\{\mathrm{ry} \mathrm{ftz-lacZ}\}$ ) or an alternative $3^{\text {rd }}$ chromosome balancer (TM3-Sb, $\mathrm{P}\{\mathrm{w}[+] \mathrm{Ubx}-\mathrm{lacZ}\})$. In the next step, male and virgin female $\mathrm{F} 1$ offspring were mated in order to generate F2 offspring flies containing the $2^{\text {nd }}$ chromosome balancer (CyO P $\{r y \mathrm{ftz}-\mathrm{lacZ}\}$ ) and the $3^{\text {rd }}$ chromosome balancer (TM3, Ser[1]). These flies were then collected to generate stable fly stocks. Similarly, for the insulin-like peptide mutant dllp6[41] which is localized at the $\mathrm{X}$ chromosome, the balanced mutant was adopted to combine the mutant with Stim-Tdl flies. 


\subsection{Molecular Biology}

\subsubsection{Molecular cloning}

Molecular techniques were performed according to standard protocols using, the methods, media and buffers described in book- Molecular cloning (Green and Sambrook, 2012).

\subsubsection{Preparation of chemical competent bacteria and their transformation}

Briefly, a single colony of the $\mathrm{DH} 5 \alpha$ strain was grown in a miniculture with $3 \mathrm{~mL}$ Lysogeny Broth $(L B)$ medium at $37^{\circ} \mathrm{C}$ for 8 hours. An appropriate volume of miniculture bacteria solution was inoculated into $0.5 \mathrm{~L}$ LB medium in a $2 \mathrm{~L}$ Erlenmeyer flask and grown at $18^{\circ} \mathrm{C}$ to an optical density (OD) $(600 \mathrm{~nm})$ value of 0.6 . The cultured bacteria were transferred into sterile $500 \mathrm{~mL}$ centrifugation tubes, incubated on ice (10 $\mathrm{min}$ ) and spun down by centrifugation (10min at $4000 \mathrm{rpm}$ ) in pre-cooled Sorvall centrifuge (Sorvall ${ }^{R}$ RC5B Refridged SuperSpeed centrifuge, Du Pont Instruments, Wilmington, United States) equipped with a GS-4 rotor. The bacterial pellet was resuspended in $80 \mathrm{ml}$ of ice-cold TB (Transformation Buffer, refer to Molecular Cloning) buffer by gentle pipetting using a pipet boy. After $10 \mathrm{~min}$ incubation on ice, bacterial were centrifuged (10 min at $4.000 \mathrm{rpm})$. The bacterial pellet was gently resuspended in $20 \mathrm{ml}$ of ice-cold TB buffer. After resuspension, 1.4 $\mathrm{mL}$ of ice cold (Dimethyl sulfoxide) DMSO (cell culture grade, Sigma-Aldrich, Cat. \#: D2650) was drop-wise added to the bacterial suspension to generate transformationcompetent bacteria. After $10 \mathrm{~min}$ incubation on ice, the competent bacteria solution was distributed into aliquots ( $100 \mu \mathrm{L}$ in $1.5 \mathrm{ml}$ Eppendorf tube), snap frozen in liquid nitrogen and stored at $-80^{\circ} \mathrm{C}$. The transformation efficiency of the competent bacteria was tested with a standard plasmid transformation assay.

Aliquots of competent bacteria were defrozen on ice. $5 \mu \mathrm{L}$ of the solutions that contain plasmids or ligated DNA fragments were added to the competent bacteria and the solution gently mixed by flicking. Then, the mixture of competent cells and exogenous DNA was incubated on ice for $30 \mathrm{~min}$ and subsequently transferred into a water bath to provide a $30-45 \mathrm{sec}$ heat shock at $42^{\circ} \mathrm{C}$. The mixture put on ice, and $900 \mu \mathrm{L}$ SOC (Super Optimal broth with Catabolite repression) medium (without antibiotics) were added. Subsequently the mixture was cultured $\left(37^{\circ} \mathrm{C}, 1 \mathrm{~h}\right)$. After this incubation, $200 \mu \mathrm{L}$ of cultured medium was transferred on a solid LB medium plate, which contained specific antibiotics for which plasmid bearing bacterial clones 
provide resistance. LB plates with transformed bacteria were cultured overnight at $37^{\circ} \mathrm{C}$.

\subsubsection{Colony picking, E.coli culture and plasmid isolation}

Bacterial clones were individually picked with autoclaved pipet tips and released into $5 \mu \mathrm{L}$ of a $1 \times$ (Phosphate-buffered saline) PBS autoclaved solution in $200 \mu \mathrm{L}$ tubes. 1 $\mu \mathrm{L}$ of the resulting solution was used to inoculate $5 \mathrm{~mL}$ LB medium containing the appropriate antibiotics and bacteria were grown overnight at $37^{\circ} \mathrm{C}$. Mini-amount plasmid preparations were done with QIAGEN Plasmid Minikit according to the protocol provided by the manufacturer (Qiagen, Hilden, Germany, Cat. \#: 12123). The plasmid samples were first checked by DNA restriction analysis (see 2.2.1.3) and then sent for sequencing (MWG) to verify that the DNA fragment of interest had been cloned. If sequencing results were confirmed, stored miniculture samples were used for large-scale culture (in $100 \mathrm{~mL}$ LB medium) and medi-amount plasmid preparation according to protocol provided by the manufacturer protocol (Qiagen, Cat. \#: 12143). Sequencing primers used for sequencing are listed in the table 3.

\subsubsection{DNA restriction analysis and ligations}

At least $1 \mu \mathrm{g}$ plasmid DNA or individual DNA fragments were digested with a given restrictive enzyme (1 1 unit in $20 \mu \mathrm{L}$ of the appropriate reaction buffers) for $1-2 \mathrm{~h}$ incubation at temperatures suggested by manufactures (NEB, Ipswich, United States). Candidate DNA fragments were separated by agarose gel electrophoresis in 1xTBE-Buffer system (90mM Tris-borate, 1mM EDTA; pH 8.3). Agarose pieces containing the ethidium bromide stained DNA fragments of choice were cut from the gel after visualization under ultra violet light (UVsolos TS Imaging system, Biometra; Analytik Jena AG, Jena, Germany). DNA fragments were eluted using QIAquick Gel Extraction Kit (Qiagen, Cat. \#: 28706). Vector backbone fragments and DNA fragments of interest were ligated with T4 ligase enzyme (NEB) in a molar ratio of 1:3 in a $20 \mu \mathrm{L}$ volume. The reactions were either left at $16^{\circ} \mathrm{C}$ overnight or at room temperature for 10 minutes. The enzymatic activity was stopped by heat inactivation at $65^{\circ} \mathrm{C}$ for 10 minutes.

\subsubsection{Vector construction of pUASTattB-Stim RNAi1 resistant cDNA RA}

The strategy and procedure designed for the molecular cloning of the Stim-RNAi1 resistant cDNA construct, was kindly provided by Dr. J. Baumbach. First, the StimRNAi1 targeted sequence (344bp, in green capital letters) was altered into a RNAi resistant sequence by replacing the nucleotides in the third position of each amino acid code into a different nucleotide (in green capital letters) that codes for the same 
amino acid that is on the basis of the Drosophila codon usage (see http://www.kazusa.or.jp/codon/cgibin/showcodon.cgi?species=7227). Together with the adjacent sequence (in bold black letter) and convenient restriction enzyme cutting sites at the 5' (Kpnl) and the 3' end (Ahdl) (shown in red capital letters), respectively (a Stim-RNAi1-resistant modified cDNA RA fragment (812bp, refer to sequence showed below) was custom-synthesized and cloned into cloning vector pEX-A (Eurofins MWG Operon). The Stim cDNA RA vector prepared by the Berkeley Drosophila Genome Project (BDGP) (Rubin et al., 2000) was obtained as a Stim cDNA RA donor vector (LD45776) from the Drosophila Genomics Resource Center (DGRC). The wild type fragment of the Stim cDNA RA vector was replaced with the Stim-RNAi1 resistant cDNA RA fragment (cut at the 5' $\mathrm{Kpnl}$ and 3'Ahdl sites) to generate the Stim RNAi1 resistant cDNA RA modified donor vector. Finally, both the wild type Stim cDNA RA and Stim-RNAi1 resistant modified cDNA RA were cut by Bglll and Xhol and cloned into the corresponding sites of pUASTattB vector (Bischof et al., 2007) to generate the wild type Stim cDNA RA and the Stim RNAi1 resistant cDNA RA modified transgenes, respectively. Related plasmids refer to table 2.

\section{Sequence of synthetic Stim-RNAi1-resistant modified cDNA RA fragment} 5'gaaactGGTACCcgctggaaagactacatattggtaacactgttgcttagtgctattattggttgttggtac gcctatcagcaaaataagaatgccaaacggcatctgcgtcgaatggcccaggatatggagggattgcaga gggctgagcaaagtctacaggagatgcagaaggaactagaacgg GCAAGGATGGAACAAGAG AACGTAGCTACTAGAAGCTTGACTTAGAACGACGACTTAAGGAGGCACCGACAC TGAGCTCTTCCAATTCCGACTTAGAGGTCCAACAACTCAAGAAAGAGATTGAAAT GTTACGGAATGAGTTATCGCGGGCGGAGTTTGAACTGGTTGATAATTGTTGGTC TCCACCCCCGCAGCTCCAGTCTTGGCTACAGTATACCTACGAGCTTGAGAGAAA AAACCACCAAAAGAAACGGACCTCCGCGGAAAAACAACTCCAATCCGCGAGGG AAGCGTGCGAAAAGTTACGCAAAAAGCGCTCTAGCTTAGTCGGAGCCTTTGTCT CGacgcacggaaagagtattgatgatgtggatcggtcgattgttgaggcacggaatgcctcggagatgta acaaacgagctgcaagaacgactgcatcgctggaagcaaatcgagacgtgccttggcttaaacattgtgaa caacaatggtctgccetacttggagaatgttctgtacggtcgaaatgggggcttacaaagttccatgggcatg agttcaaccaagggttctagagcacgtattaccaacagcaccgaagacctgGACGATGAGTCcataca 3'

Table 2 Information of plasmids

\begin{tabular}{|l|c|c|c|}
\hline $\begin{array}{l}\text { Stock } \\
\text { Number }\end{array}$ & Construct & Vector Backbone & $\begin{array}{c}\text { Reference/ } \\
\text { Source }\end{array}$ \\
\hline YX460a & $p E X-A-$ Stim-RNAi1 resistant modified \\
$c D N A R A$ & $p E X-A(2450 \mathrm{bp})$ & $\begin{array}{c}\text { Eurofins MWG } \\
\text { Operon }\end{array}$ \\
\hline
\end{tabular}




\begin{tabular}{|l|c|c|c|}
\hline YX461a & pOT2-Stim cDNA RA & pOT2(1665bp) & $\begin{array}{c}\text { (Rubin et al., } \\
\text { 2000) } \\
\text { BDGP: } \\
\text { LD45776 }\end{array}$ \\
\hline YX462 & $\begin{array}{c}\text { LD_pOT2_Stim RNAi1 resistant cDNA } \\
\text { RA modified }\end{array}$ & pOT2(1665bp) & This study \\
\hline RK416 & pUASTattB LSD-1-PC:EGFP & pUASTattB (8489bp) & I. Bickmeyer \\
\hline YX463 & pUASTattB_Stim RNAi1 resistant cDNA & pUASTattB (8489bp) & This study \\
\hline YX464 & RA modified & & \\
\hline
\end{tabular}

\subsubsection{Single fly genotyping by polymerase chain reaction (PCR)}

Single flies carrying a mutation and wild type control flies (w[1118]) were transferred into a $1.5 \mathrm{~mL}$ Eppendorf tube (Eppendorf, Hamburg, Germany) snap frozen with liquid nitrogen. $50 \mu \mathrm{L}$ squishing buffer (10mM Tris-HCl pH8.2, 1mM EDTA, 25mM $\mathrm{NaCl} ; 200 \mu \mathrm{g} / \mathrm{mL}$ Proteinase K (freshly added, Sigma-Aldrich, Cat. \#: P6556100MG)) were added. Flies were homogenized in squishing buffer with a pipet tip and incubated $\left(30 \mathrm{~min}\right.$ at $\left.37^{\circ} \mathrm{C}\right)$ on a rotation wheel for $2 \mathrm{~min}$ at $95^{\circ} \mathrm{C}$ and snap centrifuged. $1 \mu \mathrm{L}$ of the crude extract and $1.5 \mu \mathrm{L}$ of PCR primers $(10 \mathrm{pmol} / \mu \mathrm{L})$ were used for $20 \mu \mathrm{L}$ PCR reactions using the QIAGEN Hot Start Master Mix (Qiagen, Cat. \#: 203443) or the Phire Tissue Direct PCR 2X Master Mix (ThermoFisher Scientific, Cat. \#: F170L). Information about the primers used to identify the different gene sequences see table 3 .

Table 3 PCR primers for sequencing or genotyping

\begin{tabular}{|l|l|l|l|}
\hline $\begin{array}{l}\text { Target } \\
\text { Gene }\end{array}$ & $\begin{array}{c}\text { Stock } \\
\text { Number }\end{array}$ & Sequence / Identity & Reference \\
\hline SP6 & 5' ATTTAGGTGACACTATAG 3' & $\begin{array}{l}\text { https://www.addgene.org/mol- } \\
\text { bio-reference/sequencing- } \\
\text { primers/ }\end{array}$ \\
\hline $\begin{array}{l}\text { Stim } \\
\text { exon6 } \\
\text { up }\end{array}$ & RKO825 & 5' GTCATCGGACTCGCTCAGAT 3' & R. P. Kühnlein \\
\hline $\begin{array}{l}\text { Stim } \\
\text { exon6 } \\
\text { down }\end{array}$ & RKO816 & 5' ATCTGAGCGAGTCCGATGAC 3' & R. P. Kühnlein \\
\hline $\begin{array}{l}\text { Stim } \\
\text { exon10 }\end{array}$ & RKO825 & 5' ACTAGAACGGGCCAGAATGG 3' & R. P. Kühnlein \\
\hline
\end{tabular}




\begin{tabular}{|l|l|l|l|}
\hline $\begin{array}{l}\text { down } \\
\text { pUAST } \\
\text { SV40 } \\
\text { (pUAST } \\
\text { up) }\end{array}$ & RKO19 & 5' AGAAGTAAGGTTCCTTCACAA 3' & MWG-Biotech \\
\hline $\begin{array}{l}\text { pUAST } \\
\text { Hsp70 } \\
\text { (pUAST } \\
\text { low) }\end{array}$ & RKO20 & 5' ACTGAAATCTGCCAAGAAGTA 3' & MWG-Biotech \\
\hline $\begin{array}{l}\text { dAkh } \\
\text { up }\end{array}$ & MGO944 & 5' ACCTTCTCGCCGGGCAAG 3' & (Gáliková et al., 2015) \\
\hline $\begin{array}{l}\text { dAkh } \\
\text { down }\end{array}$ & MGO945 & 5' ATTGGCACGATCGGTTGGGT 3' & (Gáliková et al., 2015) \\
\hline $\begin{array}{l}\text { dFOXO } \\
\text { [delta94] } \\
\text { up }\end{array}$ & JBO807 & 5'TTGCCGCTGACAATTATGATCAAG & (Slack et al., 2011) \\
\hline $\begin{array}{l}\text { dFOXO } \\
\text { [delta94] }\end{array}$ & JBO808 & 5' AAGGTAGTGCCTATGATCCAG 3' & (Slack et al., 2011) \\
\hline
\end{tabular}

\subsubsection{RNA-seq analysis of adult fly fat body tissues}

Abdominal fat body tissues of adult male flies at day 11 after Stim-Ttl On/Off induction were dissected in cold PBS buffer, snap frozen by liquid nitrogen and immediately stored at $-80^{\circ} \mathrm{C}$. Then, dissected fat body tissues of about 20 flies (at day 10 or 11) were pooled as one biological replicate per condition of each Stim-TtI On/Off experiment. Three biological replicates of three experiments were used for total RNA isolation from the fat body tissue using a commercial extraction kit according to a protocol provided by the manufacturer (Norgen BIOTEK, Cat. \#: 36200). Typical RNA samples contained at least $1 \mu \mathrm{g}$ of RNA with concentrations around 50ng/ $\mu \mathrm{L}$ (DVD Appendix 1.). RNA quality was checked with Agilent 2100 Bioanalyzer (Agilent Technologies, Santa Clara, United States) (DVD Appendix 2.). PolyA-containing mRNAs of each sample were enriched, fragmented, and then reverse transcribed into adaptor barcoded cDNA libraries using the TruSeq Stranded mRNA Library Prep Kit (Illumina). Libraries from 3 biological replicates per condition were normalized and pooled. Samples were subsequently sequenced with Illumina HighSeq2500 (100 bp paired end reads, and around 30 million sequencing depth). Sequencing data were filtered, trimmed and mapped to the annotated Drosophila melanogaster genome (dmel-all-gene-r6.06.fasta.gz, FlyBase, http://flybase.org/), which allows counting reads per genes by CLC bio (Qiagen, Hilden, Germany) (DVD Appendix 2.). Standard gene expression analysis was performed to quantify 
differential gene expression using the DESeq2 (Love et al., 2014) analysis package (https://www.bioconductor.org/) based on R language. Genes expressed with a $\log _{2}$ fold change $>0.5, p<0.05$ were classified as differentially expressed genes (Wald test, Please see DVD Appendix 5.). RNA sample quality analysis, library preparation, sequencing, sequencing data trimming, mapping, reads counting, and gene differential expression analysis were performed by the Max Planck Genome center Cologne, Germany (http://mpgc.mpipz.mpg.de/home/). RNA data quality analysis (Clustering analysis (Euclidean distances) and principal component analysis (PCA) was performed by Yanjun Xu with DESeq2 based on $R$ ( $R$ version 3.3.1, run in x86_64-apple-darwin13.4.0 platform, OS X El Captain version 10.11.6) (R Core Team, 2016). R studio as an integrated development environment for $R$ was used (RStudio, 2015). DESeq2 (1.12.4) analysis was done based on the vignette of DESeq2 as well as one essential workflow of DESeq2 (Vignette: https://bioconductor.org/help/package-vignettes/; Workflow: http://www.huber.embl.de/users/klaus/Teaching/DESeq2Predoc2014.html) (DVD Appendix 4 and 6).

Gene Ontology (GO) enrichment analyses of up-regulated and down-regulated genes were carried out by FlyMine (Lyne et al., 2007) with non-adjusted $p$ value < 0.05 (http://www.flymine.org/), up or down-regulated gene list with Gene ID names was pasted into identifier window to establish a official gene list, and "analysis" button was pressed for different analysis. GO term analysis items were checked and corresponding GO term lists were exported). Three kinds of GO terms were analyzed separately, those for biological process (BP) (DVD Appendix 7.10.), for cellular compartment (CC) (DVD Appendix 8.11.) and for molecular function (MF) (DVD Appendix 9.12.). Enriched $G O$ terms with the corresponding $p$ values were summarized and clustered based on semantic similarities by REVIGO with default setting (Supek et al., 2011), and further visualized by R (R studio) and Adobe Illustrator CS5.1 (Adobe Systems, San Jose, United States), respectively (Similarly, corresponding GO term lists (GO term NO, non adjusted $p$ value) were passed into REVIGO identifier window respectively, With default setting, "Start Revigo" was pressed for analysis. Corresponding $\mathrm{R}$ scripts were exported for $\mathrm{R}$ visualization (DVD Appendix 13-18, basic knowledge about $R$ Graphics is required). Then images were saved as EPS files, which were further modification (font, image size). Representative and specific GO terms were shown in the figures. 


\subsubsection{Reverse Transcription and quantitative PCR (RT-qPCR)}

\subsubsection{SYBR® green I RT-qPCR}

RT-qPCR consists of three steps which are (i) the sample preparation and RNA extraction, (ii) reverse transcription and (iii) real-time quantitative Polymerase Chain Reaction. The different steps were performed according to instructions of manufacturers.

\section{(i) Sample preparation and RNA extraction}

For whole fly RNA extraction, 10 adult male flies from one experiment were collected in a $2 \mathrm{~mL}$ tube and considered as one experimental and biological replicate. For isolated heads or abdomens, 20 adult male fly heads from one experiment were cut on dry ice and transferred in a $2 \mathrm{~mL}$ collection tube (VWR, Cat. \#: 732-3598), representing one experimental and biological replicate. For fat body tissue, 12-15 adult male abdominal fat body tissues from one experiment were dissected and collected into $0.5 \mathrm{ml}$ collection tube (Biolabproducts, Cat. \#: 21-19-650) as one experimental biological replicate. Sequentially, three experimental replicates in total were collected for RNA extractions.

Quick RNA MicroPrep Kit (Zymo Research, Cat, \#: R1054) was used for RNA extractions. Sterile ceramic beads were sorted into each fly collection tube $(2 \mathrm{~mL}$ tube: 1 cylindrical bead (peqlab, 91-PCS-CK68P); 0.5ml tube: 15-18 1.4mm round ball beads (peqlab, 91-PCS-CK14)). RNA-lysis-buffer was then transferred to each tube ( $2 \mathrm{ml}$ tube: $600 \mu \mathrm{L}, 0.5 \mathrm{~mL}$ tube: $300 \mu \mathrm{L}$ ). Homogenization was carried out with the mixer mill (MM400, Retsch, 30s ${ }^{-1}, 3 \times 45 s$ ). The homogenized lysate was transferred to $1.5 \mathrm{~mL}$ Eppendorf tubes, and centrifuged for $1 \mathrm{~min}$ at $>12000 \mathrm{x} \mathrm{g}$. A distinct volume of the supernatant ( $2 \mathrm{~mL}$ tube: $450 \mu \mathrm{L}, 0.5 \mathrm{~mL}$ tube: $200 \mu \mathrm{L}$ ) was transferred to a fresh RNAase-free $1.5 \mathrm{~mL}$ Eppendorf tube, and mixed well with same volume of $100 \%$ RNAase-free ethanol by vibrate shaking. The mixed solution was loaded into a Zymo-Spin IC Column, and the solution was centrifuged (1 min, at $>12000 \times \mathrm{g}$ ) into the $2 \mathrm{~mL}$ collection tube. The flow-through was discarded. The column containing the RNA was washed with $400 \mu \mathrm{L}$ RNA-Prep buffer, and centrifuged at $>12000 \times \mathrm{g}$ for $1 \mathrm{~min}$. The flow-through was discarded again. The column was centrifuged twice by adding $700 \mu \mathrm{L}$ and $400 \mu \mathrm{L}$ RNA-Wash buffer, respectively, followed each time by centrifugation ( $1 \mathrm{~min}$ at $>12000 \mathrm{xg}$ ). Finally, the column was centrifuged for an additional $2 \mathrm{~min}$, $>12000 \mathrm{xg}$ to completely remove residual ethanol. The column was finally inserted into RNAase-free $1.5 \mathrm{~mL}$ 
Eppendorf tube and RNA was eluted with water (whole fly: $30 \mu \mathrm{L}$, fly head or fat body tissue: $15 \mu \mathrm{L}$ ) by centrifugation ( $1 \mathrm{~min}$ at $>12000 \times \mathrm{g}$ ), and the spin through containing the RNA was transferred on ice immediately. RNA concentration and purity was measured with the NanoDrop1000 $(260 / 230=2.0-2.2)$ and the RNA samples were stored at $-80^{\circ} \mathrm{C}$ for later use.

\section{(ii) Reverse transcription}

Same amounts of RNA (whole fly or head: $1.000 \mathrm{ng}$; fat body tissue: $500 \mathrm{ng}$ ) were used for reverse transcription with the QuantiTect Reverse Transcription Kit (Qiagen, Cat. \#: 205311). The RNA samples were adjusted to a final volume of $12 \mu \mathrm{L}$ to which $2 \mu \mathrm{L}$ genomic (gDNA) of Wipeout buffer (7x) in $500 \mu \mathrm{L}$ Eppendorf tubes. The mixed solution was incubated at $42^{\circ} \mathrm{C}$ for $2 \mathrm{~min}$ to eliminate genomic DNA from the samples. Afterwards, reverse transcription tubes were immediately transferred to ice. Then, 4 $\mu \mathrm{L}$ RT Buffer (5x), $1 \mu \mathrm{L}$ Primer Mix and $1 \mu \mathrm{L}$ of Reverse Transcriptase was sequentially added to the reverse transcription tubes and gently mixed by pipetting. The reverse transcription reaction was done by incubating at $42^{\circ} \mathrm{C}$ for $15 \mathrm{~min}$, at $95^{\circ} \mathrm{C}$ for 3min, cooled at $4^{\circ} \mathrm{C}$ with PCR machine (Biorad, Cat. \#: 186-1096SP1 or Applied Biosystems, GeneAmp@ PCR system 9700). The cDNA was diluted with 1:25 ratio, and quickly stored at $-20^{\circ} \mathrm{C}$.

(iii) qPCR primer design and real-time quantitative PCR (qPCR)

For the primer design, sequences of the target gene exons were taken from FlyBase (http://flybase.org/). To define the forward and reverse primer which allow the amplification of two adjacent exons (exonA and exonB), a 200 bp 3' end exonA sequence and a $200 \mathrm{bp} 5$ ' end of the exonB sequence were merged, and the merged sequences were entered into the PrimerQuest Tool (http://eu.idtdna.com/Primerquest/Home/Index) to identify a suitable primer set searching with default setting. Then, the suggested primer sets containing forward primer targeting exon1 sequence and reverse primer targeting exon2 were chosen and tested for their specificity. For this, primer sequences were blasted in FlyBase to ensure an alignment score of larger than 40 to exclude unspecific binding. For primers obtained from published references, their targeting specificities were also checked as mentioned above. The chosen primers ordered from MWG-biotech (eurofin_Genomics, http://www.mwg-biotech.com/) for synthesis, checked by PCR with control sample cDNA templates using the QIAGEN Hot Start Master Mix (Qiagen, Cat. \#: 203443) to ensure that no unspecific DNA fragments were amplified. Finally, amplification efficiencies of candidate primers were checked by using diluted 
original control cDNA sample (ratio: 1:5, 1:25, 1:125, 1:625) as templates. Two technical replicates were used for efficiencies check. Only primer sets with a good efficiency $(>0.9 ;<1.1)$ were used for the qPCR experiments.

qPCR reactions were carried out with three technical replicates of normalization standards, Act5C and /or Ribosomal protein L32 (RpL32), serving as internal controls and references (Bauer et al., 2009; Ponton et al., 2011). To start qPCR reactions, diluted cDNA templates from reverse transcription $(4 \mu \mathrm{L})$ were transferred into 0.1 mL strip-tubes (Qiagen, Cat. \#: 981103), into Micro Amp Fast Optical 96 well reaction plate (Applied Biosystems, Cat. \#: 4346906) or into 96 Well Plates, Semi-Skirted, FAST (Starlab, Cat. \#: E1403-7700) which were kept on ice. Then master mix solutions to amplify sequences of interest were prepared by mixing primer DNA, water, and SYBR (Rotor-Gene SYBR Green PCR Kit (2000), Qiagen, Cat. \#: 204076). For MWG synthesized primers, forward and reverse primers were diluted in RNAse-free water to a concentration of $10 \mathrm{pmol} / \mu \mathrm{L}$, additional RNAase-free water (3 $\mu \mathrm{L})$, and SYBR $(10 \mu \mathrm{L})$ were added to form $16 \mu \mathrm{L}$ of master mixes; or qPCR reaction using primers ordered from QIAGEN, the reaction mixture were adjusted to contain the forward and reverse primer mixes $(2 \mu \mathrm{L})$, SYBR $(10 \mu \mathrm{L})$ and $16 \mu \mathrm{L}$ of master mixes.

For the qPCR amplification reactions, tube-stripes or plates were loaded onto the Qiagen Rotor-Gene Q System or Applied Biosystems Step One Plus ${ }^{\mathrm{TM}}$ Real-Time PCR System. The reactions were performed according to a standard protocol which was an incubation for $5 \mathrm{~min}$ at $95^{\circ} \mathrm{C}$ followed by 40 amplification cycles $\left(5 \mathrm{~s}\right.$ at $95^{\circ} \mathrm{C}$ followed by $10 \mathrm{~s}$ at $60^{\circ} \mathrm{C}$ ). When double strands DNA fragments were produced from PCR, SYBR Green I dyes bind with dsRNA and emit fluorescence (Qiagen, Hilden, Germany), which was detected by the PCR system. Raw data analyses were conducted by corresponding setup software to get averaged Cycle Threshold $\left(C_{T}\right)$ values from the triplicates data. Relative gene expression quantification was done with Microsoft Excel 2013 following the $2^{-\Delta \Delta C T}$ method (Livak and Schmittgen, 2001; Schmittgen and Livak, 2008).

\subsubsection{TaqMan RT-qPCR}

Taqman RT-qPCR also requires RNA extraction, reverse transcription (RT) and realtime qPCR (Chen et al., 2005). As with SYBR-qPCR, three experimental biological replicates and corresponding technical replicates were used. It is noteworthy that 
there are major differences with respect to the source materials used and experimental manipulations.

15 adult male fly abdomens were cut for RNA extraction on dry ice. QIAZOL (Qiagen, Cat. \#: 79306) and QIAGEN miRNA extraction kit (Qiagen, Cat. \#: 217004) was used to extract whole RNA including different kinds of small RNAs e.g. 2s ribosome RNA ( $r R N A$ ) and siRNA generated from the short hairpin RNA transgenes carried by the flies. Homogenization was done as described in 2.2.4.1. Whole RNA extraction was carried out based on the manufacturer's protocol.

Stem loop-specific RT primers for small RNA were used. They were designed to bind the 3' end of the small RNAs such as siRNAs to specifically transcribe target siRNAbased cDNA. The second difference to SYBR-qPCR was the small RNA-specific TaqMan probe in real-time qPCR, which contains the fluorescent marker at the 5' end of the probe and a non-fluorescence quencher dye at the 3 ' end. The intact dye molecule does not emit fluorescence since the quencher dye suppresses the reporter dye from emitting fluorescence (Förster-type energy transfer) (Chen et al., 2005; Förster, 1948). During qPCR amplification with small RNA specific qPCR primers, the DNA polymerase cleaves the TaqMan probes which are hybridized to the target sequence and separates the reporter dye from the quencher dye, resulting in fluorescence from the reporter dye. The increase in fluorescence during qPCR amplification is detected by PCR system and can be quantified.

In terms of $A k h R$ siRNA-specific RT primers, $A k h R$ siRNA specific qPCR primers and AkhR siRNA specific TaqMan probe (Thermofisher Scientific, Custom TaqMan® Small RNA Assay, Cat. \#: 4440418) were designed and produced by Applied Biosystems (ThermoFisher Scientific, www.thermofisher.com) based on AkhR shRNA RNAi transgene targeted sequence: TCCGCTGATCATGTTCATCTA (Perkins et al., 2015). Together with AkhR siRNA and 2s rRNA (Applied Biosystems, ThermoFisher Scientific, Cat. \#: 4427975)-specific primer sets, TaqMan® MicroRNA Reverse Transcription Kit (Applied Biosystems, ThermoFisher Scientific, Cat. \#: 4366596) and TaqMan ${ }^{\circledR}$ Universal PCR Master Mix II (Applied Biosystems, Cat. \#: 4440040) were used for RT and qPCR. The protocol of TaqMan ${ }^{\circledR}$ Small RNA Assays (ThermoFisher Scientific, www.thermofisher.com) was followed for these experiments.

During the RT reactions, 10ng RNA templates were denatured and then cDNA was 
prepared by using PCR machine as described in 2.2.3.2. 96 Well Plate (Starlab, Cat. \#: E1403-7700) and Applied Biosystems Step One Plus ${ }^{\mathrm{TM}}$ Real-Time PCR System and the standard TaqMan qPCR program was used for qPCR reactions and signal detections. Raw data analyses were conduced by setup software to get averaged Cycle Threshold $\left(\mathrm{C}_{\mathrm{T}}\right)$ values from the triplicates data. AkhR siRNA levels were normalized to the value of $2 s$ rRNA, serving as an internal standard. Relative gene expression quantification was done with Microsoft Excel 2013 following the 2- $\triangle \Delta C T$ method (Livak and Schmittgen, 2001; Schmittgen and Livak, 2008). For qPCR primer information see table 3 .

Table 4 RT-qPCR primers

\begin{tabular}{|c|c|c|c|}
\hline $\begin{array}{l}\text { Target } \\
\text { gene }\end{array}$ & $\begin{array}{l}\text { Stock } \\
\text { Number }\end{array}$ & Sequence / Identity & Reference \\
\hline RpL32 & RKO677 & QT00985677 & www.qiagen.com \\
\hline Act5c & $\begin{array}{l}\text { RKO744 } \\
\text { RKO745 }\end{array}$ & $\begin{array}{l}\text { 5` GTGCACCGCAAGTGCTTCTAA 3`} \\
5 ` \text { TGCTGCACTCCAAACTTCCAC 3` }\end{array}$ & (Bauer et al., 2009) \\
\hline $\begin{array}{l}\text { Stim-RNAi } \\
\text { RNA }\end{array}$ & $\begin{array}{l}\text { YXO1119 } \\
\text { YXO1120 }\end{array}$ & 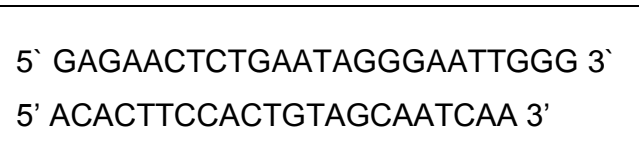 & This study \\
\hline Stim & RWO713 & QT00923020 & www.qiagen.com \\
\hline$A k h R$ siRNA & YXO1194 & 5`TCCGCTGATCATGTTCATCTA 3` & $\begin{array}{l}\text { www.thermofisher.com } \\
4440418\end{array}$ \\
\hline$A k h R$ & RKO719 & QT00931210 & www.qiagen.com \\
\hline Akh & MGO1009 & QT00957859 & www.qiagen.com \\
\hline tobi & RKO969 & QT00982646 & www.qiagen.com \\
\hline $\mathrm{CCHa} 2$ & $\begin{array}{l}\text { YX01075 } \\
\text { YX01076 }\end{array}$ & $\begin{array}{l}\text { 5' CCCGTCAGGTGCTTTACAAA 3' } \\
\text { 5' CGGAATTGGCCAAGGGATAA 3' }\end{array}$ & (Gáliková et al., 2017) \\
\hline ImpL2 & $\begin{array}{l}\text { YX01042 } \\
\text { YX01043 }\end{array}$ & $\begin{array}{l}\text { 5' AAGAGCCGTGGACCTGGTA 3' } \\
\text { 5' TTGGTGAACTTGAGCCAGTCG 3' }\end{array}$ & $\begin{array}{l}\text { Based on (Kwon et al., } \\
\text { 2015) }\end{array}$ \\
\hline$d 4 E B P$ & $\begin{array}{l}\text { JBO753 } \\
\text { JBO754 }\end{array}$ & $\begin{array}{l}\text { 5' CATGCAGCAACTGCCAAATC 3' } \\
\text { 5' CCGAGAGAACAAACAAGGTGG 3' }\end{array}$ & (Fuss et al., 2006) \\
\hline bmm & YXO1169 & QT00964460 & www.qiagen.com \\
\hline $\begin{array}{l}\text { CG7461 } \\
(d A C A D V L)\end{array}$ & $\begin{array}{l}\text { RKO746 } \\
\text { RKO747 }\end{array}$ & $\begin{array}{l}\text { 5' CTGCCACATCCAGTCATCATAG 3' } \\
\text { 5' GATCCAAGATTCCAGTCGTAGTC } \\
\text { 3' }\end{array}$ & This study \\
\hline CPT1 & YXO1155 & 5' TTCTCAACTTCCGTCGACTTATC 3' & This study \\
\hline
\end{tabular}




\begin{tabular}{|c|c|c|c|}
\hline & YX01156 & 5' GTACGTTCATACTGCCAGGAG 3' & \\
\hline $\begin{array}{l}\text { blw } \\
(d A T P 5 A)\end{array}$ & $\begin{array}{l}\text { YX01161 } \\
\text { YX01162 }\end{array}$ & $\begin{array}{l}\text { 5' AGCGCTGAGATCTCCAACAT 3' } \\
\text { 5' TGTCGGGCTCCAAGTTAAG 3' }\end{array}$ & This study \\
\hline $\begin{array}{l}\text { ATP syn } \beta \\
(d A T P 5 B)\end{array}$ & $\begin{array}{l}\text { YX01163 } \\
\text { YX01164 }\end{array}$ & $\begin{array}{l}\text { 5' CGTCGATGTCCAGTTTGATG 3' } \\
\text { 5' AGTGCGCACGGTATTTTCTC 3' }\end{array}$ & This study \\
\hline aralar1 & $\begin{array}{l}\text { YX01195 } \\
\text { YX01196 }\end{array}$ & $\begin{array}{l}\text { 5' TCCTGGGACTCTTTTCCGAATC 3' } \\
\text { 5' GCCTGGAACTCCGAGAAGGA 3' }\end{array}$ & This study \\
\hline Mdh1 & $\begin{array}{l}\text { YX01181 } \\
\text { YX01182 }\end{array}$ & $\begin{array}{l}\text { 5' GGAATGGAGCGAAAGGATCTG 3' } \\
\text { 5' GCACCTTGACGTCCTTCTTG 3' }\end{array}$ & This study \\
\hline Mdh2 & $\begin{array}{l}\text { YX01197 } \\
\text { YX01198 }\end{array}$ & $\begin{array}{l}\text { 5' CGCCGATCTGTCGCATATC 3' } \\
\text { 5' CACCGGGTTGGTGATGATGG 3' }\end{array}$ & This study \\
\hline Got1 & $\begin{array}{l}\text { YXO1199 } \\
\text { YX01200 }\end{array}$ & $\begin{array}{l}\text { 5' TAACCACGAGTATTTGCCAGTG 3' } \\
\text { 5' ACATTGCGATTCAGTTGTGTGT 3' }\end{array}$ & This study \\
\hline Got2 & $\begin{array}{l}\text { YX01201 } \\
\text { YX01202 }\end{array}$ & $\begin{array}{l}\text { 5' TGTCACGGAAGCCTTCAAGAA 3' } \\
\text { 5' GTCCAGACTACGGCTCACCA 3' }\end{array}$ & This study \\
\hline CanA-14F & $\begin{array}{l}\text { YX01165 } \\
\text { YX01166 }\end{array}$ & $\begin{array}{l}\text { 5' CGCAGCACAATATCGACAAAGG 3' } \\
\text { 5' ATCCGCGCAGGTGAGTTTA 3' }\end{array}$ & This study \\
\hline$d P G C-1$ & $\begin{array}{l}\text { YX01061 } \\
\text { YX01062 }\end{array}$ & $\begin{array}{l}\text { 5' AGACGTGCCTTCTGTCGTTC 3' } \\
\text { 5' AGCTTCGAAAGAGTCCTCGAC 3' }\end{array}$ & (Chartier et al., 2015) \\
\hline Trh & $\begin{array}{l}\text { YX01063 } \\
\text { YX01064 }\end{array}$ & $\begin{array}{l}\text { 5' CCTCCTACCAGAATGCCTACTA 3' } \\
\text { 5' CGACAGGACTTCAACACTCATC 3' }\end{array}$ & This study \\
\hline 5-HT7 & $\begin{array}{l}\text { YX01065 } \\
\text { YX01066 }\end{array}$ & $\begin{array}{l}\text { 5' AATGATTCTGAGGCTCGAAGA 3' } \\
\text { 5' TATGAGCAACCCAGTGCTGA 3' }\end{array}$ & (Becnel et al., 2011) \\
\hline NPF & $\begin{array}{l}\text { YX01067 } \\
\text { YX01068 }\end{array}$ & $\begin{array}{l}\text { 5' GCGAAAGAACGATGTCAACAC 3' } \\
\text { 5' TGTTGTCCATCTCGTGATTCC 3' }\end{array}$ & (Shankar et al., 2015) \\
\hline Dh44 & $\begin{array}{l}\text { YX01069 } \\
\text { YX01070 }\end{array}$ & $\begin{array}{l}\text { 5' GCAGGCAAATGAAGGAGAAC 3' } \\
\text { 5' CCACGTTCTTCAGGATGG 3' }\end{array}$ & $\begin{array}{l}\text { (Cavanaugh et al., } \\
2014)\end{array}$ \\
\hline Crz & $\begin{array}{l}\text { YX01071 } \\
\text { YX01072 }\end{array}$ & $\begin{array}{l}\text { 5' GACTCACGGATCTCTACGATTTG 3' } \\
\text { 5' TCTACTCGGTTGGCATTGAAG 3' }\end{array}$ & (Gáliková et al., 2017) \\
\hline Dsk & $\begin{array}{l}\text { YX01073 } \\
\text { YX01074 }\end{array}$ & $\begin{array}{l}\text { 5' CCGATCCCAGCGCAGACGAC 3' } \\
\text { 5' TGGCACTCTGCGACCGAAGC 3' }\end{array}$ & (Williams et al., 2014) \\
\hline Dh31 & $\begin{array}{l}\text { YX01078 } \\
\text { YX01079 }\end{array}$ & $\begin{array}{l}\text { 5' CGACCTACTCATGGAACTGATG 3' } \\
\text { 5' CAAAGTCCACGGTTCGTTTG 3' }\end{array}$ & This study \\
\hline SIFa & $\begin{array}{l}\text { YX01107 } \\
\text { YX01108 }\end{array}$ & $\begin{array}{l}\text { 5' TTCACACTCACTCTGCTCCTG 3' } \\
\text { 5' TGTTGCGTTTGCCGAAGATG 3' }\end{array}$ & This study \\
\hline Lst & RKO1106 & QT00948185 & www.qiagen.com \\
\hline Hex-C & $\begin{array}{l}\text { YX01203 } \\
\text { YX01204 }\end{array}$ & $\begin{array}{l}\text { 5' GCGGAGGTGCGAGAACTTAT 3' } \\
\text { 5' CCACTTCCAGGCAAAAGCGA 3' }\end{array}$ & $\begin{array}{l}\text { Fly Primer Bank: } \\
\text { PP11731 }\end{array}$ \\
\hline FAS & YXO1187 & 5' CTCCACCATCGAGGAGTTCA 3' & (Mattila et al., 2015) \\
\hline
\end{tabular}




\begin{tabular}{|c|c|c|c|}
\hline & YXO1188 & 5' CTTGAGCTTGCCAATCCTGT 3' & \\
\hline \multirow{2}{*}{$A C S$} & YXO1189 & 5' TGTCGCATTTGAAGCGAGTGA 3' & Fly Primer Bank: \\
\hline & YXO1190 & 5' CCACCAGTAATCGCGGTCAT 3' & PP26971 \\
\hline \multirow{2}{*}{ Lipin } & YXO1191 & CACACCGACAACACACTGGA & Fly Primer Bank: \\
\hline & YXO1192 & СТTCTTCTCGCССTGAAACAG & PP22555 \\
\hline \multirow{2}{*}{ sug } & YXO1170 & 5' CCAGTCCGTGATCATGAAGGCTC 3' & \multirow{2}{*}{ (Mattila et al., 2015) } \\
\hline & YXO1171 & 5' GTGCCAGTGCATCCAAGGTGTCG 3' & \\
\hline \multirow{2}{*}{ daw } & YXO1185 & 5' CAAGCGGGAGTACTATGCCC 3' & \multirow{2}{*}{ (Mattila et al., 2015) } \\
\hline & YXO1186 & 5' CCGGGATGGTTGTAGCTGAG 3' & \\
\hline \multirow[t]{2}{*}{$m d y$} & RKO746 & 5` AAGACAGGCCTCTACTATTGT 3` & (Baumbach et al., \\
\hline & RKO747 & $5{ }^{\prime}$ CCCATCATGCCCATAAATGCC $3^{\prime}$ & 2014b) \\
\hline
\end{tabular}

\subsection{Biochemical experiments}

\subsubsection{Bicinchoninic acid (BCA) and Bradford protein assay}

Adult flies were paralyzed by $\mathrm{CO}_{2}$ and collected based on gender. After snap freezing with liquid nitrogen, fly homogenates (5 adult male fly per technical replicate, 3-6 technical replicates) were generated in $2 \mathrm{~mL}$ tubes (VWR, Cat. \#: 732-3598) or $1.2 \mathrm{~mL}$ collection tubes (Qiagen, Cat. \#: 19560; Caps: Cat. \#: 19566) or $0.5 \mathrm{~mL}$ collection tube (Biolabproducts, Cat. \#: 21-19-650) by homogenization with one metal beads (Askubal korntal-München, Cat. \#: 50492) or 15-18 $1.4 \mathrm{~mm}$ round ball beads (peqlab, 91-PCS-CK14)) in a mixer mill (MM400, Retsch, Hann, Germany). For the bicinchoninic acid assay (BCA), flies were homogenized in $600 \mu \mathrm{L}$ homogenization buffer $\left(0,05 \%\right.$ Tween in $\left.\mathrm{H}_{2} \mathrm{O}\right)$ and incubated at $70^{\circ} \mathrm{C}$ (water bath) for $5 \mathrm{~min}$. The detailed procedure is also described in (Hehlert, 2016). Quantification of the total protein with the BCA assay is based on colorimetric detection of the biuret reaction by using reagent containing bicinchoninic acid. $50 \mu \mathrm{L}$ homogenates of each replicate and BSA (Bovine serum albumin) protein standard samples (0, 25, 125, 250, 500, $750 \mathrm{mg} / \mathrm{mL}$ ) were transferred to 96-well micro-test plate (Sarstedt, Cat. \#: 82.1581) and the background absorbance of each sample was measured at $570 \mathrm{~nm}$ with a micro-test plate reader (Bio-Rad, Bench mark). Then, $200 \mu \mathrm{L}$ of BCA mixed-reagents (BCA Protein Assay Kit, Pierce, Thermo Scientific; Cat. \#: 23225) was added to the homogenate and incubated at $37^{\circ} \mathrm{C}$ for $30 \mathrm{~min}$ with mild rotation. BCA reaction absorbance of each sample was measured at $570 \mathrm{~nm}$. After substraction of the background absorbance, the protein concentration of each sample was calculated based on a standard curve. 
For measuring the protein concentration with the Bradford assay, 10 flies or 15 fly abdominal tissues were homogenized in 500 or $300 \mu \mathrm{L}$ tissue lysis buffer (personal communication from Dr. Yuanbin Xie; Tris- $\mathrm{HCl} 250 \mathrm{mM}, \mathrm{NaCl} 125 \mathrm{mM}$, glycerol $5 \%$ (v/v), IGEPAL CA-630 (Sigma-Aldrich, Cat. \#: I8896-50ML) 0.5\% (v/v), $\mathrm{MgCl}_{2} 1.5$ $\mathrm{mM}$, Na-deoxycholate (0.25\%, v/v), Dithiothreitol (DTT) $1 \mathrm{mM}, \mathrm{NaF} 25 \mathrm{mM}, \mathrm{Na}_{3} \mathrm{VSO}_{4}$ $1 \mathrm{mM}$, ethylene glycol-bis( $\beta$-aminoethyl ether)-N,N,N',N'-tetraacetic acid (EGTA) 2mM, EDTA 1mM, Phosphotase inhibitors 1x (Roche, Basel, Switzerland, Cat. \#: 04906837001)). The assay is based on maximum absorbance shift of an acidic reagent containing Coomassie Brilliant Blue G-250 when it binds to proteins. $100 \mu \mathrm{L}$ (1:10) diluted homogenate of each sample and protein standard samples $(0,2.5,5$, $10,15 \mu \mathrm{g} / \mu \mathrm{L}$ ) were mixed with $3 \mathrm{~mL}$ Bradford assay reagent (Sigma-Aldrich, Cat. \#: B6916_500) and incubated at room temperature for $5 \mathrm{~min}$, respectively. After that, Bradford assay reagent reaction absorbance of each sample was measured at $595 \mathrm{~nm}$ with spectral photometer (Phamacia LKB Novaspec II, Phamacia, London, United Kingdom). Protein concentrations were calculated as described for the BCA assay.

\subsubsection{Western blotting}

Based on the Bradford assay results, protein samples were normalized to a concentration of $7 \mu \mathrm{g} / \mu \mathrm{L}$ by adding lysis buffer. The normalized protein samples were mixed with an equal volume of $2 \mathrm{x}$ sample buffer (final 1x; Stock 3x: 150mM Tris [pH 6.8], 6\% Sodium dodecyl sulphate (SDS), 0.3\% Bromophenol blue, 30\% Glycerol, $300 \mathrm{mM}$ DTT and incubated at $95^{\circ} \mathrm{C}$ for $3 \mathrm{~min}$ followed by centrifugation $(13,000 \mathrm{rpm}$ for 3 min). $15 \mu \mathrm{L}$ of the supernatant (contains about $50 \mu \mathrm{g}$ protein) and $10 \mu \mathrm{L}$ of a PageRuler prestained protein ladder (Thermofisher Scientific, Cat. \#: 26616) were loaded and separated by SDS-Polyacrylamide gel electrophoresis (PAGE) gel (10\%; $0.75 \mathrm{~mm}$ loading lane) electrophoresis at $70 \mathrm{~V}$ for $30 \mathrm{~min}$, and $150 \mathrm{~V}$ for $60 \mathrm{~min}$ (BioRad, Hercules, United States). After separation, proteins were transferred from the gel onto nitrocellulose membrane by a wet electro-transfer with $300 \mathrm{~mA}$ for $1 \mathrm{~h}$ on ice at $4^{\circ} \mathrm{C}$ (PowerPAC 3000, Bio-Rad, Hercules, United States). After the transfer, the membrane was washed in 1x Tris-buffered saline (TBS) buffer at room temperature for $5 \mathrm{~min}$ and blocked in blocking solution (1x Tris-buffered saline with $0.1 \%$ Tween20 (TBST)+5\% (Albumin bovine fraction) BSA (Biomol, Cat. \#: 014001)) at room temperature for $1 \mathrm{~h}$ with mild shaking. Afterwards, the membrane was incubated with one of diluted primary antibody solutions (1xTBST $+5 \% \mathrm{BSA})$ at $4{ }^{\circ} \mathrm{C}$ overnight and reacted with primary antibodies: rabbit anti-Phospho-Drosophila Akt Ser505 (1:500, 
NEB, Cat. \#4054S), rabbit anti-Phospho-Akt Thr342 (1:500, PhosphoSolutions, Cat. \#: p104-342), rabbit anti-Akt (1:500, NEB, Cat. \#: 9272S) or mouse anti- $\beta$ tublin (Hybridoma Bank, Cat. \#: E7) in a successive order (see below). After three washes with 1XTBST buffer (each 5min), the membrane was incubated with secondary antibody solutions (1xTBST+5\%BSA) at room temperature for $1 \mathrm{~h}$ using goat antirabbit-HRP (1:2000, Pierce, Cat. \#: 31460) or goat anti-mouse-HRP (1:10000, Pierce Cat. \#31430). After five washes in 1xTBST buffer (each 10min), the membrane was finally incubated for $3 \mathrm{~min}$ in Western ${ }^{\mathrm{TM}}$ Bright quantum chemiluminescent reagentsmixed solution (Biozym, Cat. \#: K-12042-D10) at room temperature in the dark. After removal of the solution, the membrane was wrapped in a plastic foil and fixed. The chemiluminescence of horseradish peroxidase (HRP)-conjugated secondary antibody/primary antibody/antigen protein complexes was detected by exposing the membrane to a Kodak BioMax XAR Film (Sigma-Aldrich, Cat. \#: 1651454). Images of the exposed film were scanned, processed and analysed by Image (1.51h). Analysis with the above mentioned antibodies was performed successively, i.e. after staining and detection of the antibody reactions, the primary and secondary antibodies were stripped of by incubating the blotted membrane in Restore western blot stripping buffer for $10 \mathrm{~min}$ at room temperature (Thermofisher Scientific, Cat. \#: 46430), and the membrane was stained with a different set of antibodies as described above. For standard Western blott buffers refer to Abcam Western blotting protocol (http://www.abcam.com/tag/western\%20blot\%20protocols).

\subsubsection{Coupled colorimetric assay (CCA) for TAG equivalents quantification}

CCA was used as described (Hildebrandt et al., 2011). CCA is based on the detection of the dye quinoneimine, which represents the end product of the enzymatic reactions following the release of glycerol from TAGs (Hildebrandt et al., 2011). Fly homogenates for CCA were prepared as described in 2.3.1 for the BCA assay. $50 \mu \mathrm{L}$ of the supernatant of each homogenates and $50 \mu \mathrm{L}$ of a triglyceride standard solutions (with different concentrations) were each transferred into a 96-well micro-test plate and measured at $540 \mathrm{~nm}$ for background absorbance. Then, the homogenates and the triglyceride standard solutions (Biomol, Cat. \#: Cay-10010509; $0,5.5,11,22,33,44 \mu \mathrm{g}$ in $50 \mu \mathrm{L}$ homogenization buffer) were mixed with $200 \mu \mathrm{L}$ (Triglycerides; Microgenics, Fremont, United States, Cat. \#: 981786) and incubated at $37^{\circ} \mathrm{C}$ with mild shaking for final reaction absorbance measurement at $540 \mathrm{~nm}$. After background absorbance subtraction, the concentration of the TAG equivalents of each sample was calculated based on standard curve. To get relative fat content 
value, the content of TAG equivalents was normalized to protein content determined with the BCA assay.

\subsubsection{Glycogen assay}

To determine the glucose and glycogen content of the flies, homogenates were prepared as described in 2.3.1 for BCA assays. Glycogen were done based on protocol described in (Gáliková et al., 2015; Tennessen et al., 2014).The whole procedure (except final incubation) was carried out on ice to avoid an unspecific degradation of glycogen (personal experience). The quantification of Glycogen is based on the detection of free glucose and the released glucose from glycogen hydrolysis with Amyloglucosidase (Sigma-Aldrich, Cat. \#: A1602-25MG). The free glucose and total glucose amount were determined with Glucose Assay (Glucose oxidase, GO) Kit (Sigma-Aldrich, Cat. \#: GAGO20-1KT). The procedure was essentially done by following a standard protocol of the laboratory (Baumbach et al., 2014a; Hehlert, 2016; Tennessen et al., 2014). $30 \mu \mathrm{L}$ of diluted supernatant (1:3-1:5) of the initial homogenate of each sample was measured at $540 \mathrm{~nm}$ for background absorbance and then mixed with $100 \mu \mathrm{L}$ of GO Assay reagent in 96-well microtest plate (Sarstedt, Cat. \#: 82.1581) to determine the free glucose content. In parallel, another $30 \mu \mathrm{L}$ supernatant of each sample was also measured at $540 \mathrm{~nm}$ for background absorbance, and then mixed with $100 \mu \mathrm{L}$ of GO Assay reagent supplemented with $0.3 \mathrm{U}$ amyloglucosidase in 96-well microtest plates for released and free glucose determination. Glycogen standard samples (Glycogen standard: Sigma-Aldrich, Cat. \#: G0885; 0, 0.3, 0.6, 1.2, 2.4, $4.8 \mu \mathrm{g} / \mu \mathrm{L})$ were also measured to establish a standard concentration curve. After 30 min incubation (shaking at $37^{\circ} \mathrm{C}$ ) and reaction termination (by adding $100 \mu \mathrm{L}$ of $12 \mathrm{~N} \mathrm{H}_{2} \mathrm{SO}_{4}$ ), the final reaction absorbance was measured at 540nm. After the subtraction of background absorbance, the TAG equivalents concentration of each sample was calculated on the bases of the values of the standard curve. To compare, the glycogen contents of differently treated flies, the glycogen were normalized to the protein content obtained from BCA assay of the respective flies.

\subsubsection{Thin layer chromatography (TLC) for neutral lipids determination}

TLC involves the differential distribution of molecules in the stationary phase during the capillary force-driven mobile phase migration (Stahl, 1983). TLC of neutral lipids was carried out using the procedures described in (Baumbach et al., 2014b; Hehlert, 2016). Briefly, 5 adult male flies were homogenized in lipid lysis buffer (150 $\mu \mathrm{L}$ 
methanol, $75 \mu \mathrm{L}$ chloroform and $60 \mu \mathrm{L} \mathrm{H}_{2} \mathrm{O}$ ) with a pestle in a homogenizer (VWR, Cat. \#: 431-0100, and 4314-009). During the subsequent $1 \mathrm{~h}$ incubation at $37^{\circ} \mathrm{C}$ in a water bath, lipids were enriched into the chloroform phase. Additional $75 \mu \mathrm{L}$ chloroform and $75 \mu \mathrm{L} 1 \mathrm{M} \mathrm{KCl}$ solutions were added. Then, fly lipids in chloroform phase were separated from the aqueous phase by centrifugation at 3,000 rpm for 2 min, and the chloroform phase was transferred into a new tube. Chloroform was evaporated in a SpeedVac concentrator (SpeedVac ISS110 system, ThermoFisher Scientific, Waltham, United States), and the remaining lipid pellet was resolved in 100-200 $\mu \mathrm{L}$ of elution solution (chloroform/methanol; 1:1) and loaded on high performance thin layer chromatography (HPTLC) plates (Merck, Darmstadt, Germany, Cat. \#: 1.05721.0001). To identify the different neutral lipid classes, Lipid Standard, Mono-, Di-, Triglyceride Mix (SUPELCO, Sigma-Aldrich, St. Louis, United States, Cat. \#: 1787-1AMP) and fatty acids (FAs) (Fluka, HoneyWell Research Chemicals, Seelze, Germany, Cat. \#: 85679) were used as markers (TAG, DAG, MAG: $2.5,10,30,60 \mu \mathrm{g}$ in $20 \mu \mathrm{l}$, FA: 1, 2, 4, $6 \mu \mathrm{g}$ in $20 \mu \mathrm{l}$ ) for neutral lipid identification and quantification. Normally, one fly equivalent of lipids was resolved in $25 \mu \mathrm{L}$ elution buffer and loaded onto the chromatography plates; when flies contain more than $100 \mu \mathrm{g}$ TAG equivalents as determined by CCA, however, only half the amount was used. Separation of the lipids was achieved by dipping part of HPTLC plate into running solution (hexane/diethylether/acetic acid (70:30:1, v/v/v; Merck) in sealed chamber so that the mobile phase level is below the positions of loaded lipid samples. After the separation, the air-dried plate was quickly immersed in $8 \%(\mathrm{w} / \mathrm{v})$ $\mathrm{H}_{3} \mathrm{PO}_{4}$ containing $10 \%$ (w/v) copper (II) sulfate pentahydrate. The different neutral lipid classes were visualized by heating the plate in an oven at $180^{\circ} \mathrm{C}$ (Gerhardt, Königswinter, Germany). Charred TLC plates were scanned and the different lipid classes were quantified using the lipid standards curve and ImageJ (1.51h). Experiments were repeated twice. First round experiment was kindly done by the Bachelor student Annika Franziska Borcherding (Borcherding, 2014).

\subsection{6 ${ }^{14} \mathrm{C}$ incorporation lipogenesis assay}

Lipogenesis assays were done according to the protocol established by (Katewa et al. 2012) and (Hehlert, 2016). Briefly, two groups of Stim-Tdl 4-5 day old adult male

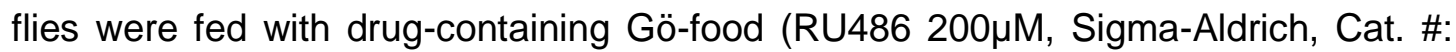
M8046-500MG), and control food (ethanol, 200uM), respectively, for 4-5 days, before they were transferred back to the normal Gö-food for another 4 days of feeding. The two groups of flies were then transferred to Gö-food, which contained ${ }^{14} \mathrm{C}$-labeled 
glucose (dissolved in Perkin Elmer, Cat. \#: NEC042X050UC), dissolved in ethanol; each $1 \mathrm{~mL}$ Gö-food mixed with $18.3 \mu \mathrm{L}{ }^{14} \mathrm{C}$ glucose solution. 100 flies were kept in a medium-size vial with about 200 ul food. After 24 hs feeding, at least 80 flies were frozen in liquid nitrogen ( $0 \mathrm{~h}$ sample in at least 4 replicates of 20 flies each per replicate) using a $1.5 \mathrm{~mL}$ Eppendorf tube. These samples were used for the following lipid fractionation.

Lipid fractionation was according to the solid phase extraction method described by (Kaluzny et al.1985). Flies were homogenized in $0.5 \mathrm{~mL}$ chloroform with pestle in a motor-driven homogenizer (VWR, Cat. \#: 431-0094 and 431-0100). DSC-NH2 columns (PK30 DISCOVERY DSC-NH2 6mL Tube 1GM, Sigma-Aldrich, Cat. \#52640-U,) were pre-equilibrated with $2 \mathrm{~mL}$ chloroform. The homogenates were transferred onto columns. The following lipid fractions were eluted sequentially with 3 $\mathrm{mL}$ chloroform: 2-propanol (2:1) solution to elute the neutral lipids (TAGs, DAGs, cholesterol). After washing the column with $3 \mathrm{~mL}$ diethyl ether containing $32 \%$ acetic acid, $3 \mathrm{~mL}$ methanol was used to elute the phospholipids and fatty acids. The solvents were evaporated under nitrogen. The dried neutral lipids as well as the combined phospholipid and fatty acids fractions were dissolved in $4 \mathrm{~mL}$ scintillation liquid (Ultra Gold scintillation cocktail, Perkin Elmer, Waltham, USA, Cat. \#: 6013326) and scintillation samples were transferred into $4 \mathrm{~mL}$ plastic scintillation bottles. Radioactivity (counts per minute, CPM) was measured in a scintillation counter (TriCarb 2100TR, Packard, Perkin Elmer; each sample for 4min counting). Except labeled, other related basic chemical reagents were purchased from Merck. Data were analysed with Excel 2011. Experiments were repeated twice.

\subsection{Microscopy, staining, and imaging}

\subsubsection{Dissection, microscopy and imaging of adult flies}

Adult male flies were anesthesized with $\mathrm{CO}_{2}$. Their wings and legs were manually removed with a forceps (World Precision Instruments, Sarasota, United States, Cat. \#: 500085), the fly bodies were transferred into a $2 \mathrm{~mL}$ Eppendorf tube and kept on ice for $10 \mathrm{~min}$. The fly bodies were put on metal plate together with a plastic ruler, which were magnified by Zeiss Stereomicroscope Discovery V8 microscopy (Carl Zeiss AG, Jena, Germany) and imaged by a Canon EOS 5D Mark II camera (Canon, Tokyo, Japan). Image analysis was performed with ImageJ (1.51h). Experiments were repeated at least twice. 
For abdominal fat body tissue imaging and dissection, the fly bodies were further fixed on a silica gel pad (MPlbpc workshop, Göttingen, Germany) using an insect needle (MPlbpc workshop, Göttingen, Germany; diameter $=0.1 \mathrm{~mm}$, fly thorax lateral part was penetrated for fixation). After that, the fixed fly body was immersed in icecooled PBS (1x) buffer. Fly abdomens were opened with a microscissor (World Precision Instruments, Cat. \#: 14124). Gut and reproduction organs were removed with forceps, and the abdominal fat body tissues were exposed for imaging and further dissection. Gut ring fat body tissue, and dorsal carcass A1 segment or the spermatheca attached fat body tissue, as showed in abdominal fat body tissue scheme (Figure 1), were freshly dissected for a subsequent in vivo staining.

\subsubsection{Ex vivo staining of adult fat body cells and confocal imaging}

\subsubsection{Ex vivo staining of dissected fat body tissues}

Dissected fat body tissues were immersed for $5 \mathrm{~min}$ in staining solution on a microscope cover slide $(20 \times 40$ or $20 \times 60 \mathrm{~mm}$, No.1, MARIENFELD laboratory glassware, VWR, Radnor, United States, Cat. \#: 631-0654). The staining solution contained the following fluorescent dyes diluted in PBS buffer: LD540 for lipid droplet staining (1:500, $4 \mu \mathrm{g} / \mathrm{mL}$, excitation/emission wave length: 514 / 540-570 nm; gift from C. Thiele (Spandl et al., 2009)), CellMask for cell membrane staining (1:1000, $5 \mathrm{mg} / \mathrm{mL}$, excitation/emission wave length: 633 / 635-670 nm, Invitrogen, ThermoFisher Scientific, Cat.\# C10046) and DAPI for nuclear staining (1:1000, excitation/emission wave length: 405/415-470, Invitrogen, ThermoFisher Scientific, Cat. \#: D1306). After staining, the fat tissues were washed with PBS buffer once, and covered by small round cover glass slides (diameter $=10 \mathrm{~mm}$, Menzel, ThermoFisher Scientific, Cat. \#: 631-1340). The brinks of cover slides were then sealed by quick dry nail polishing oil (quikDry top coat, essence, cosnova Beauty, Sulzbach, Germany). At least 3 flies were dissected and analysed by imaging.

\subsubsection{Confocal imaging of ex vivo stained dissected fat body tissues}

Confocal images were took with the Zeiss Laser Scanning Microscope (LSM) 780 confocal microscope system (C-Apochromat 40x/1.20 W Korr. M27 objective, excitation/emission filter as indicated in 2.4.2.1, fixed gain, pixel size: 2048 x 2048, 1 optical stack in Z-axis direction). Fluorescence signals were detected by a PMT (photomultiplier) detector and analysed with the Zen software. Experimental and control samples were imaged with same laser power, gain, detection time per pixel, and bits per pixel ( 8 bits). In addition, transmission light channel imaging (wide field 
imaging) was used for identify the different tissues. Experiments were repeated at least twice. First round experiment was kindly done by the Bachelor student Annika Franziska Borcherding (Borcherding, 2014).

\subsubsection{Fat body cell and lipid droplet size quantification}

Single stack images of optical section of the fat body tissue were used for analysis, and LD540, CellMask, (4',6-diamidino-2-phenylindole) DAPI channel images were merged by ImageJ $(1.51 \mathrm{~h})$.

Intact fat body cells, which have the DAPI signal marking their nuclei but no apparent lipid droplet fusions were selected. Cell area sizes based on CellMask signals were quantified with the polygon selection tool, and the LD540 signal channel image of selected cells were duplicated and saved for lipid droplet size quantification. The quantification strategy and procedure were based on an earlier technique developed by Dr. Philip Hehlert (Hehlert, 2016) with only minor modifications.

To exclude disturbing signals of cells, images (cells of interest, LDs image channel) were first copied into a new image with black background $(1,000 \times 1,000$ pixels for normal cell size). The black background images were further checked by alternative lookup table - HiLo, which was used to find optimal minimum displayed value (same displayed value thresholds were set for same round, same day TI On/Off fat body images, image pixels were applied with pseudocolors in minimum displayed range, middle displayed range, and maxium displayed range, respectively). After that, the black background images were processed by "Gaussian blur" with sigma (radius) 2.00 for more smooth signals. Subsequently, the smoothed images were processed with "Enhance Local Contrast" (Contrast Limited Adaptive Histogram Equalization, CLAHE) for better local contrast (block size: 127, histogram bins: 256, maximum slope: 3.00 , non mask, normal process). Then, the minimum displayed values of images were adjusted to optimized threshold. In the next step, signals of images were processed with s-log curve -ImageJ macro (slope=1, developed by Dr. Philip Hehlert and Andres Hertel (MPI-BPC, Göttingen, German). After this treatment, low intensity signals were increased to artificial higher intensity signals, while high intensity signals remain their values, signal-noise ratio and signal homogeneity of candidate lipid droplets (strongly and weakly stained) was improved. Moreover, the images were processed with "Gaussian blur" again. Later, binary images were generated based above step processed images (Method: Moments, Visualization: Black and white). The candidate lipid droplet areas were separated by "watershed" and verified by manual separation (Inverted picture, separate by black pencil, 2 pixel 
width). Finally, binary images with lipid droplet objects were analysed by "analyze particles" (size: $0.10 \mu \mathrm{m}$-infinity, circularity: 0.01-1, show: masks, selecting choices: Display results, clear results, Summarize, Add to Manager). This way, averaged cell area, total lipid droplet area per cell, averaged lipid droplet size per cell in each fly fat body tissue can be obtained. Moreover, averaged lipid droplet area percentage of each cell in TtI On and Ttl Off can be calculated. Data were analysed with Excel 2011 (Student's t-test).

\subsubsection{Confocal imaging of fat body expressing CaLexA GFP}

After eclosion, flies were kept at $25^{\circ} \mathrm{C}$ for 5 days. After that, abdominal deep fat body tissues (attached to spermatheca) of FB-Gal4/UAS RNAi/CaLexA virgin female flies (RNAi On) and FB-Gal4/balancer /CaLexA flies (RNAi Off) (described in 2.1.3) were dissected followed the method described in 2.4.1. The dissected fat body tissues were immersed in 1xPBS containing DAPI for nuclear staining (1:1000, excitation/emission wave length: 405 / 415-470, Invitrogen, ThermoFisher Scientific Cat. \#: D1306). Afterwards, different groups of stained fat body tissues were imaged by Zeiss LSM780 confocal microscope system with the identical optical and electrical parameters (C-Apochromat 40x/1.20 W Korr. FCS M27 objective, GFP excitation/emission filter: 488/500-530nm, fixed gain, pixel size: 1024 x 1024, 1 optical stack at Z-axis direction).

\subsubsection{5-ethynyl-2'-deoxyuridine (EdU) staining of adult fly fat body cell and midgut tissue and confocal imaging}

Flies were fed with Gö-food containing EdU $(100 \mu \mathrm{M})$ from day 5 to day 11 after StimTtI RNAi expression. Incorporation of EdU during DNA synthesis was detected through the EdU reaction with an azide fluorescent dye (Alexa). EdU staining was carried out with the Click-iT® EdU Alexa Fluor® 647 Imaging Kit according to manufacture's instruction (Molecular probes, ThermoFisher Scientific, Cat. \#: C10340). Briefly, dissected fly tissues were fixed with $3.7 \%$ paraformaldehyde in PBS solution at room temperature for $20 \mathrm{~min}$. After washing with 3\% BSA (Biomol, Cat. \#: 014001) in PBS, the fixed tissues were permeabilized with $0.5 \%$ Triton $\AA X$ 100 (Merck) in PBS (PBST) for $20 \mathrm{~min}$ at room temperature. Afterwards, having washed with $3 \%$ BSA in PBS, fly tissues were incubated with Click-IT reaction cocktail at room temperature for 30 min in the dark. After washing, cell nuclei in the tissue were stained with Hoechst $33342(5 \mu \mathrm{g} / \mathrm{mL})$ in PBS. The tissues were washed 
and embedded in Mowiol (Sigma-Aldrich, Cat. \#: 32,549_0) for confocal imaging. For confocal imaging, and processing of the images see 2.4.2.2. The excitation/emission peak wavelength of EdU and Hoechst staining dyes are 647/665 nm and 350/461 $\mathrm{nm}$, respectively.

\subsubsection{Akh and dILP-2 immunostaining and confocal imaging}

Akh and dILP-2 staining was performed as described in (Baumbach et al. 2014b). Prior to the staining, the brain, the corpora cardiaca, and the ventral cord of adult males were dissected in cold PBS and fixed in 3.7\% paraformaldehyde on ice for $2 \mathrm{~h}$. Then, tissues were washed with $0.25 \%$ Triton ${ }^{\circledR}$ X-100 (Merck, Darmstadt, Germany) in PBS for 20min at room temperature and blocked with 1\%BSA and $3 \%$ Normal Goat Serum (NGS) in $0.25 \%$ PBST at room temperature for $1 \mathrm{~h}$. Afterwards, tissues were incubated with the respective primary antibodies at $4^{\circ} \mathrm{C}$ overnight: rabbit antiAkh (diluted 1:300, (Kim and Rulifson, 2004)), rat anti-dlLP-2 (diluted 1:400, (Géminard et al., 2009)), or mouse anti-nc82 (diluted 1:50) (Developmental Studies Hybridoma Bank, lowa, United, States). After 3x10 minutes washing with 1\%BSA and 3\% (normal goat serum) NGS (Invitrogen, Thermo-Fisher Scientific, Cat. \#: 31873 ) in $0.25 \%$ PBST at room temperature, tissues were incubated with secondary antibodies: Alexa-568-goat anti-rabbit (diluted 1:1000, Invitrogen, ThermoFisher Scientific, Cat. \#: A-11036), Alexa-488-goat anti rat (diluted 1:1000, Invitrogen, Cat. \#: A-11006) and Alexa-647-goat anti-mouse (diluted 1:1000, Jackson ImmunoResearch Laboratories). The stained tissues were washed $5 \times 10$ minutes in PBST at room temperature and kept overnight in PBS at $4^{\circ} \mathrm{C}$ for extra washing. Fixed tissues were mounted in Mowiol (Sigma-Aldrich, Cat. \#: 32,549_0) for confocal imaging. For confocal imaging, a Zeiss Plan Apochromat 40x/1.4 oil DIC M27 objective was used. Other details are described in 2.4.2.2 and 2.4.4.5. The excitation/emission filter setting of Alexa 647, Alexa 568, and Alexa 488 are 633/635670nm, 568/590-625nm, 488/500-530nm, respectively. The averaged Akh staining or dILP-2 fluorescence intensities were quantified using Z-axis maximum intensity projections of image stacks of 8-10 biological replicates. Image analysis was performed with ImageJ (1.51h). Statistical analysis was done with Excel 2011 (Student's t-test). Experiments were repeated at least twice. 


\subsection{Physiological and behavior assays}

\subsubsection{Wet body weight measurement}

Five adult males were collected and taken into a $1.5 \mathrm{~mL}$ Eppendorf tube as one replicate, and 3-5 replicates were measured for each group of flies using the Sartorius Mikrowaage MC5 (Satorius AG, Göttingen, Germany). The weight of each tube (empty) with flies was also determined by averaging the values of 3 different measurements. Statistical analysis was done with Excel 2011 (Student's t-test). Each experiment was repeated at least twice. First round experiment was kindly done by the Bachelor student Annika Franziska Borcherding (Borcherding, 2014).

\subsubsection{Starvation resistance assay}

Day 21 Stim-Ttl On/Off, Stim-Ttl On negative control and Stim-Ttl On negative control male flies were used for starvation resistance experiments. In each experiment, at least 200 flies were kept in 10 small-size vials. Each vial was filled with $10 \mathrm{~mL} 2 \%$ agarose gel to provide water for flies. The assay were done at $18^{\circ} \mathrm{C}$ with a diurnal-light/dark cycle. After $48 \mathrm{~h}$ of starvation, 20 flies of each experimental fly batch were collected and frozen at $-20^{\circ} \mathrm{C}$ for later CCA assay. Starved flies, which died during early starvation, were checked and removed three times a day (i.e. in the morning, the afternoon, and the evening). The flies, which died after the median survival time were collected and stored at $-20^{\circ} \mathrm{C}$ for later CCA assay. Data processing and survival analysis were performed with Excel 2011 and online survival analysis software OASIS (Log Rank test) (Yang et al., 2011). Starvation data are based on experiments, which were repeated at least twice.

\subsubsection{Startle-induced climbing assay}

Startle-induced climbing assays were performed as described in (Gáliková et al., 2015). At least 250 Stim-TtI On/Off male flies at day 21 after Stim RNAi induction were sorted into 8 small-size vials (each vial contain around 30 flies) one day prior to being tested. The climbing assay was performed using the modified 'countercurrent distribution' method (Benzer, 1967; Shcherbata et al., 2007). After flies were repeatedly tapped onto the bottom of assay tubes, the climbing ability of adult male flies was assayed by counting the number of flies who climbed a certain height within a limited time period (30s-1min). The climbing index was calculated. For details see (Gáliková et al., 2015). Experiments were repeated at least twice. 


\subsubsection{Lifespan assay}

At least 225 Stim-Tdl On and Stim-Tdl Off male flies were sorted at day 0 into 15-18 small-size vials (each vial contained 15 flies as one replicate) for life span assay. Each vial contained $5 \mathrm{~mL}$ Gö-food. The lifespan assay was carried out at $25^{\circ} \mathrm{C}$ with diurnal-light/dark cycle $(12 \mathrm{~h} / 12 \mathrm{~h})$ and $60 \%$ relative humidity in a Percival incubator (CLF PlantClimatics, Percival Scientific, lowa, United States). The fly vials were kept at horizontal orientation. The flies were transferred every two days into new vials with fresh food without $\mathrm{CO}_{2}$ anaesthetization. Dead flies were counted ever two days at the beginning and daily when flies passed the median survival time. Data process and lifespan survival analysis were performed with Excel 2011 and online survival analysis software OASIS (Log Rank test) (Yang et al., 2011). Experiments were repeated at least twice.

\subsubsection{Capillary feeding assay}

To quantify adult fly food intake, a capillary feeding (CAFE) assay was performed according to (Ja et al., 2007) with slight modifications. One of them is that the 24-well cell culture plate was altered, i.e. the bottom of each well was replaced with a metal net to allow air ventilation. Appropriate holes were made on the cover of the plate, which were inserted with individual ring caps capillary (Hirschmann, Eberstadt, Germany, Cat. \#: 9600105) for liquid food (5\% sucrose, 5\% yeast enzymatic extract (MP Biomedicals, Santa Ana, United States, Cat. \#: 103304) in H2O) feeding. Each capillary was loaded with around $5 \mu \mathrm{L}$ liquid food. After each capillary was inserted into the individual chamber, the liquid food level was marked. On each plate, one chamber was left empty as evaporation control to be subtracted from the estimated food consumption of the flies. After one day, the food level change of each fly and the evaporation controls were monitored (ruler and/or camera imaging) to calculate the food intake volume based on $1 \mu \mathrm{L}$ length marked on the capillary. One adult male fly was kept at each chamber. For the data presented, at least 36 adult male flies were monitored for each group of the above-mentioned flies. The CAFE plates were kept at corresponding temperature (Stim-Ttl flies were assayed at $18^{\circ} \mathrm{C}$ before $\mathrm{Ttl}$, at $29^{\circ} \mathrm{C}$ during $\mathrm{Ttl}$, and at $18^{\circ} \mathrm{C}$ again after $\mathrm{Ttl}$;) in a sealed box with diurnal day/night cycle $(12 \mathrm{~h} / 12 \mathrm{~h})$. The box contained water at bottom to ensure high humidity, which is put at Percival incubator. For Stim-Ttl flies, 2 days old adult male flies after eclosion were transferred into CAFÉ chambers to adapt into new environment for 2 days. Then, their daily food intake was monitored before Stim-Ttl, during Stim-Ttl and after Stim-Ttl treatment. For Stim-Tdl flies, their daily food intake was monitored after 
Stim-Tdl. Data processing and statistical analysis were performed with ImageJ (1.51h) and Excel 2011 (Student's t-test). Experiments were repeated at least twice.

\subsubsection{Locomotor activity assay}

Spontaneuous locomotor activity of single male flies was assessed with the Drosophila activity monitor (DAM) system (DAM2, http://www.trikinetics.com/, TriKinetics, Waltham, United States,). The DAM system is based on monitoring the movements of single flies in a small, closed assay tube, which contains food at one end and a sponge foam stopper at the other. The assay tube is placed onto a DAM2 monitor system, which monitors the number of times a fly passes the midpoint of the assay tube during a five minutes period. The DAM system was placed in a Percival incubator to ensure a constant environment such as constant humidity (60\%), constant temperature $\left(18^{\circ} \mathrm{C}\right)$ and a diurnal-light/dark cycle $(12 / 12 \mathrm{~h})$. Data processing and the statistical analysis were carried out with Excel 2011 (Student's t-test). Experiments were repeated at least twice.

\subsection{7 $\mathrm{CO}_{2}$ assay}

Fly $\mathrm{CO}_{2}$ production was assayed as method described recently (Yatsenko et al., 2014). It involves the assessment of the $\mathrm{CO}_{2}$ production from five adult adult flies over 2-3h at noon and in the evening. The $\mathrm{CO}_{2}$ production rate was assayed in a manually constructed assay tube composed of a $1 \mathrm{~mL}$ pipette tip (SARSTEDT AG\&Co Nümbrecht, Germany). The tip end of the pipette was extended with a capillary (BLAUBRAND®, 25 $\mu \mathrm{L}$, Brand, Wertheim, Germany, Cat. \#: 708722), and the connection part between the extending capillary and the pipette was tightly sealed with glue (Power-Pritt-Gel, Henkel, Düsseldorf, Germany). The pipet tip was then loaded with a small piece of sponge foam and loaded with soda lime (around (1/4 volume of the pipet tip) (Sigma-Aldrich, Cat. \#: 72073-1KG). On the top of the soda lime, another small piece of sponge foam was placed to form the major part of assay tube. For $\mathrm{CO}_{2}$ production measurements, the device was placed in a Percival incubator $\left(25^{\circ} \mathrm{C}\right.$ and $60 \%$ relative humidity) to eliminate temperature differences. Five male flies of a given experimental series were transferred into the assay tube, which is well sealed with modeling clay (Künstpark, Herne, Germany) and thus presents a "closed system", and their $\mathrm{CO}_{2}$ production was determined. For statistical analysis, data were taken from at least seven assay tubes containing five flies each, and $\mathrm{CO}_{2}$ production measurements were done both at noon and in the evening (technical replicates). One assay tube was used as a negative control for the metabolic rate by 
measuring the $\mathrm{CO}_{2}$ production in an assay tube, which contains 5 dead males. To ensure a constant environment such as constant humidity $(60 \%)$, constant temperature $\left(25^{\circ} \mathrm{C}\right)$ and a diurnal-light/dark cycle, the assay device was placed in a Percival incubator. The assay tubes containing the flies were set into a frame in the sealed glass chamber. The bottom of glass chamber was filled with a diluted, red colored Eosin-solution (Sigma-Aldrich, Cat. \#: HT110232-1I) into which the capillary end of the assay tube was immersed. To measure the $\mathrm{CO}_{2}$ production, the consumption of $\mathrm{O}_{2}$ in the sealed assay tube and its conversion into $\mathrm{CO}_{2}$ by the fly metabolism was determined as follows. Flies metabolize $\mathrm{O}_{2}$ into $\mathrm{CO}_{2}$. The newly produced $\mathrm{CO}_{2}$ is constantly absorbed by the soda lime and thus removed from the air present in the sealed assay tube. The removal of $\mathrm{CO}_{2}$ in the air tube causes a reduction of the air pressure in the assay tube and, in turn, a difference in air pressure between the air in assay tube and the air in the glass chamber. Since the assay tube extends into a capillary, which is immersed into the red Eosine solution, this solution is visibly pulled in the capillary as a result of the reduced air pressure in the assay tube. The increasing volume of the Eosin solution in the capillaries was monitored every 30min by camera imaging (FujiFilm X-T10, FujiFilm, Tokyo, Japan). Images were processed with ImageJ (1.51h). Statistical analysis was performed with Excel 2011. Experiments were repeated at least twice. 


\section{Results}

Three major questions were addressed sequentially. For the first part (3.1) of the result section I used alterations of fat storage and intracellular $\mathrm{iCa}^{2+}$ levels in the fat storage organ of the fly to identify putative upstream components of the Stromal interaction molecule (Stim) activity. The second part (3.1) describes the identification and characterization of phenotypes of the Long-term Impairment of STIM function induced Obesity (LISO) after transient Stim RNAi in fat storage tissue. In the third (3.3) and fourth part (3.4), I investigated how dysfunctional energy homeostasis develops and the underlying mechanisms.

\subsection{Identification of candidate genes which act upstream of Stim and regulate body fat storage}

In a previous in vivo screen in fat storage tissue of adult Drosophila melanogaster, store-operated calcium entry (SOCE) was identified as a critical regulator of the fly's fat storage. One of the key player of the SOCE system is Stim (Soboloff et al., 2012). Knockdown of Stim expression substantially increases the body fat content of the fly (Baumbach et al., 2014a). Besides, this screen also revealed that a knockdown of the $G \gamma 1, G a q 49 B$, and $P l c 21 C$ genes expression in the fat storage tissue significantly increases the fat storage of the fly (Baumbach et al., 2014a). Moreover, loss of function mutations of $A k h$ and $A k h R$, which encodes a $G$ protein-coupled receptor that binds to Akh have been reported to cause more fat accumulation in larvae as well as in adult Drosophila fat body tissue (Bharucha et al., 2008; Gáliková et al., 2015; Grönke et al., 2007). Conversely, gain-of-function (gof) of Akh by ectopic overexpression depletes fly stored fat (Lee and Park, 2004). I therefore speculated that $G$ proteins encoded by $G y 1, G \alpha q 49 B$, and Plc $\beta$ encoded by Plc21C may transmit Akh/AkhR signalling activity to mobilize stored lipids via Stim mediated SOCE. In order to test this assumption, I systematically examined possible fat storage alterations following the functional impairment of these gene activities by confocal imaging approach.

3.1.1 Long-term and individual modulation of $A k h, A k h R, G y 1, G a q 49 B, P I c 21 C$, and Stim gene expression levels affects the fat storage of adult flies

Similar to the thermo-genetics used for the above mentioned in vivo screen, which was based on using the Gal4-UAS system (Brand and Perrimon, 1993), I performed specific manipulations of gene expression (i.e. RNAi mediated knockdown, or 
overexpression) in fat storage tissue such as the fat body tissue and midgut or in the corpora cardiaca (cc) of adult flies. For these experiments, I combined the temporal and tissue-specific switchable driver lines, i.e. the temperature inducible and fat storage tissue specific Gal4 driver provided by the TARGET system (Beller et al., 2010; McGuire, 2003) or the corpora cardiaca driver (Akh-Gal4) (Kim and Rulifson, 2004) to control expression of the UAS-RNAi or cDNA transgenes.
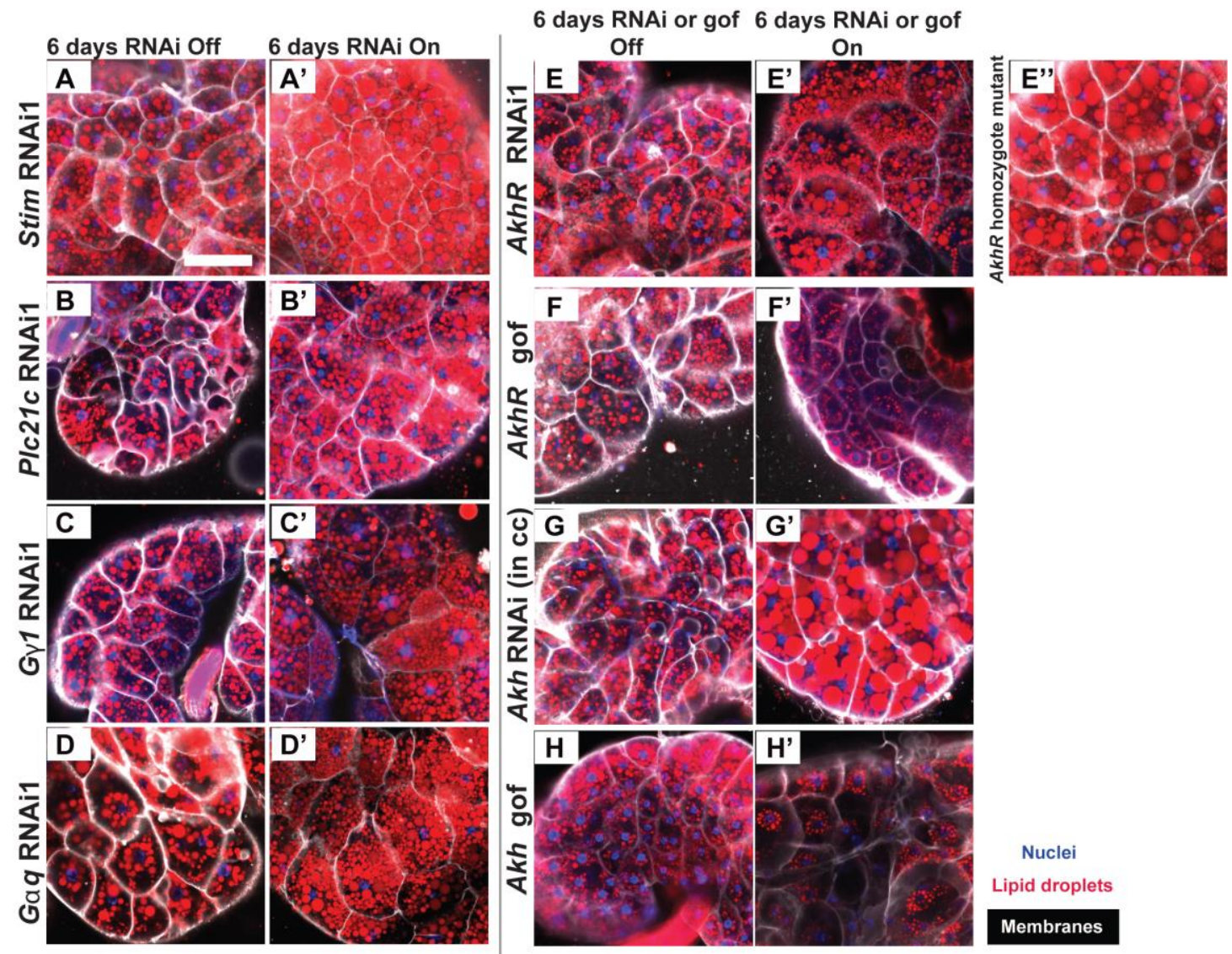

Figure 3. Long-term and individual modulation of $A k h, A k h R, G y 1, G a q 49 B, P I c 21 C$ and Stim expression affects cellular fat storage

Confocal images showing lipid ex vivo staining of fat body tissues of adult virgin female flies containing a transgene expressing either Stim RNAi (A, A'), PIc21C RNAi (B, B'), Gy1 RNAi (C, C'), Gaq49B RNAi (D, D') or $A k h R$ RNAi construct (E, E'), or lacking $A k h R$ activity due to loss-of-function mutation (E'). Also, lipid staining of fat body of flies containing a $A k h R$ gain of function (gof) cDNA transgene ( $\left.F, F^{\prime}\right)$, the Akh RNAi transgene ( $\left.G, G^{\prime}\right)$ or the Akh gain of function (gof) cDNA transgene $\left(\mathrm{H}, \mathrm{H}^{\prime}\right)$. A-H. Flies containing the indicated RNAi transgenes, an $A k h$ cDNA transgene (gof) or a $A k h R$ gof cDNA transgene (gof) which, however, were not activated ("Off"). These flies represent experimental controls for flies in which the corresponding transgenes were activated for a 6 days period ("On"). A'- $\mathrm{H}^{\prime}$ show the fat body tissues of flies after the indicated RNAi or gof transgenes construct were activated for 6 days. Note that compared to the corresponding controls, the amount of lipid droplets is increased in the fat body tissues of flies expressing RNAi transgenes of Stim (A'), PIc21C (B'), Gy1 (C'), Gaq49B (D') or $A k h R$ (E') in fat storage tissue as well as of flies expressing the Akh RNAi transgene in the corpora cardiaca (cc) (G'). Note also that the amount of lipid droplets is 
reduced in the fat body tissues of flies expressing the $A k h R\left(\mathrm{~F}^{\prime}\right)$ and $A k h\left(\mathrm{H}^{\prime}\right)$ gain of function cDNA transgene. The figure is based on Baumbach J., and Xu, Y., et al. JGG. 2014 and was prepared in cooperation with Dr. J. Baumbach. Red: lipid droplets staining (LD540), white: cell membrane staining (CellMask), blue: nuclei staining (DAPI). The scale bar in (A) represents $50 \mu \mathrm{m}$; the same magnification was used in all Figure parts. For details of the genetic constitutions of flies and experiments see 2.1.1, 2.1.2, 2.1.3, 2.4.2.

First, the efficiencies of RNAi or overexpression (in collaboration with Dr. J. Baumbach) were validated by RT-qPCR (Baumbach, et al., 2014b). To further determine whether functional impairment of Stim, Plc21C, Gy1, Gaq49B and AkhR gene expression in fat body tissue and $A k h$ gene expression in corpora cardiaca cells change the neutral lipids storage in fat body tissue, I visualized the lipid droplets (LDs) stored in fat body cells with LD540 (Spandl et al., 2009) and CellMask ex vivo staining by using confocal imaging on fat body tissues (containing attached spermatheca) of following virgin female flies. These virgin female flies included:

- Flies were subjected to an acute knockdown of Stim, Plc21C, Gy1, Gaq49B, and $A k h R$ in fat body tissue as well as $A k h$ expression in the corpora cardiaca (6 days RNAi On),

- Flies were exposed to over-expression of $A k h R$ and $A k h$ (6 days gof On),

- Flies carried an AkhR loss-of-function mutation, as well as their control flies (6 days RNAi Off).

In comparison with the appropriate control flies (RNAi Off; (Figure 3A-E, and 3G), cellular fat accumulations, which are represented by likely more lipid droplets (LDs) accumulation in fat body cells, were increased after acute impairment of Stim, Plc21C, Gy1, Gaq49B, AkhR and Akh function (6 days RNAi On), respectively (Figure 3A'-E', and 3G'). Conversely, $A k h R$ or Akh overexpression (Figure 3F' and $\left.3 H^{\prime}\right)$ markedly reduced the LDs storage of fat body cells as compared to control flies (Figure $3 \mathbf{F}$ and $3 \mathbf{H}$ ). These data indicate that similar body fat storage alterations occurred when Stim, Plc21C, Gy1, Gaq49B, AkhR and Akh gene activities were functionally impaired. The results show that altered Stim, PIc21C, Gy1, Gaq49B, and $A k h R$ gene activities conversely correlate with the cellular fat storage, and they suggest the existence of a link between Akh/AkhR signalling, G protein, Plc $\beta$ and SOCE activities and fat metabolism. 
3.1.2 Long-term and individual down-regulation of $A k h, A k h R, G y 1, G a q 49 B$, PIc21C, and Stim expression levels results in a decrease of cytoplasmic calcium levels of the fat body cells

Since I proposed that $A k h / A k h R$ signalling affects fat storage through a G protein/PIC $\beta / S T I M$ mediated SOCE pathway, I therefore expected that intracellular calcium level $\left(\mathrm{iCa}^{2+}\right)$ can be influenced by a knockdown of the expression of Stim, Plc21C, Gy1, Gaq49B or $A k h R$. To investigate $\mathrm{iCa}^{2+}$ changes of fat body cells, I used the CaLexA system to visualize the long term cytosolic calcium levels via the calcium-responsive expression of membrane targeted GFP (Masuyama et al., 2012). I further combined the CaLexA system with the fat body-specific RNAi targeting Stim, Plc21C, Gy1, Gaq49B, and $A k h R$ expression. To avoid possible developmental effects of the gene Knockdowns, pre-adult stages of flies were kept at $18^{\circ} \mathrm{C}$, to reduce the activity of the Gal4-UAS system to a minimum level. Six days after the hatching of the flies, experimental (RNAi On) and control (RNAi Off) flies were exposed for six days to 25 ${ }^{\circ} \mathrm{C}$ to activate the RNAi transgene expression and the CaLexA system.

Consistent with previous findings (Baumbach et al., 2014a), and similar to the phenotype observed following functional impairment of Stim (Figure 4A"), the knockdown of its candidate upstream genes, i.e. Plc21C, Gy1, Gaq49B, and AkhR, considerably decreased the calcium responsive expression of membrane targeted GFP, showing that the iCa ${ }^{2+}$ level of fat body cells is reduced (compare Figure 4B", C', D', E' with Figure 4B', C', D', E'). Taking also the results of the cellular fat storage changes (Figure 3) into account, these data suggest that Akh/AkhR/G protein/PIc $\beta / S T I M$ signalling affects $\mathrm{iCa}^{2+}$ levels of cells, which control their fat storage.

Overall, the results further support the hypothesis that Akh/AkhR, G protein, Plc, and STIM act in the same pathway to regulate $\mathrm{iCa}^{2+}$ levels, and eventually cause fat mobilization in wild type fat body tissue. 

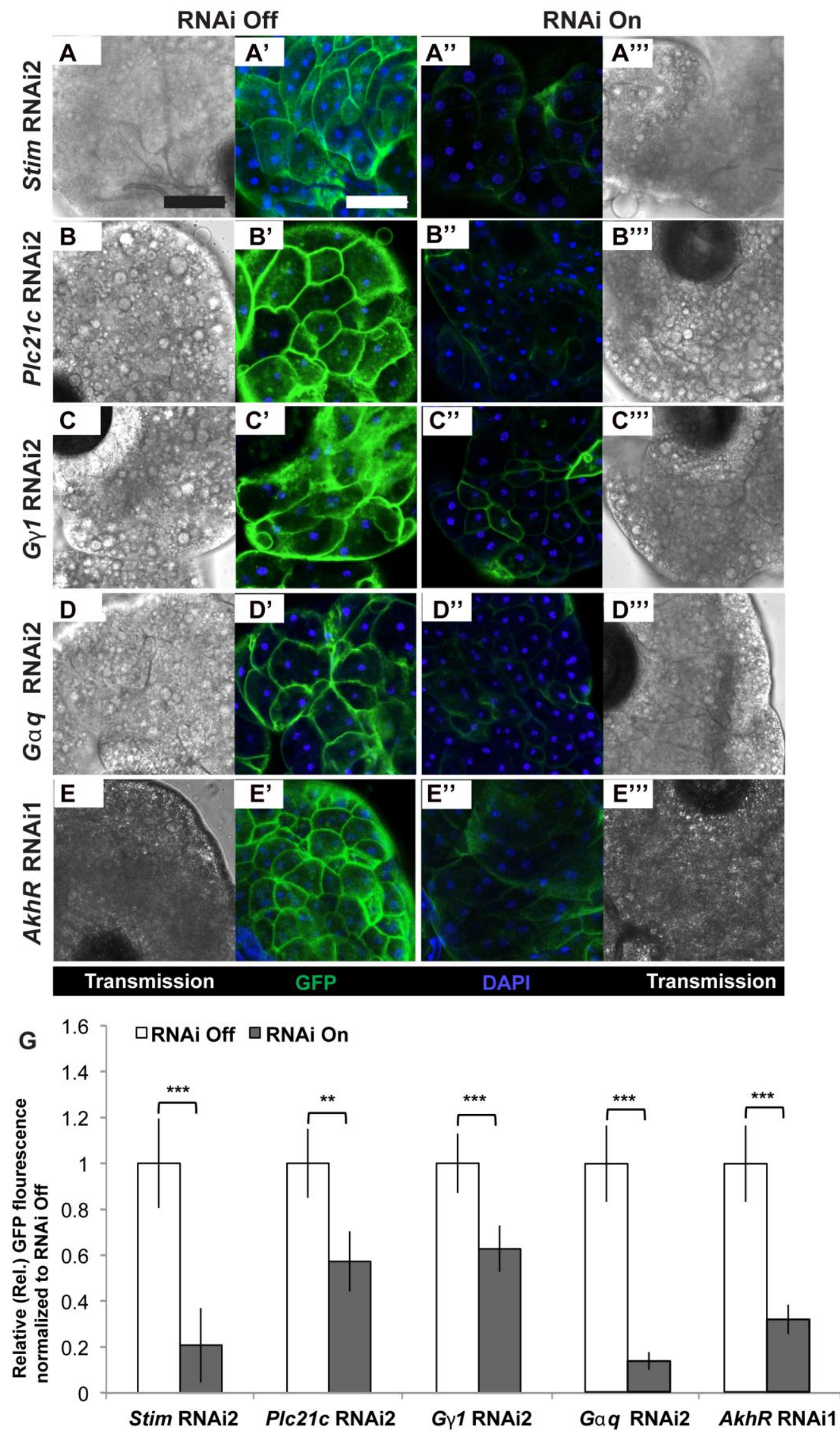

Figure 4. Long-term and individual down-regulation of AkhR, Gy1, Gaq49B, PIc21C, and Stim expression reduces cytoplasmic calcium levels of fat body cells 
(A-E, A'-E', A"-E", A'"-E"') show fat body tissue images (transmission and fluorescence micrographs) of flies expressing Stim RNAi (A, A', A", A"'), PIc21C RNAi (B, B', B", B"'), Gy1 RNAi (C, C', C", C'”), Gaq49B RNAi (D, D', D", D'”) and AkhR RNAi (E, E', E', E'") respectively, as well as a cytosolic calcium reporter CaLexA transgene. (A-E, A'"-E'") show confocal transmission channel images of adult virgin female fat body tissues. (A'-E', A"'-E') show merged images of cytoplasmic calcium channel (Green, membrane targeted GFP from CaLexA transgenes) and nuclei (blue, DAPI staining). ( $\left.A^{\prime}-E^{\prime}\right)$ show the fat body cells of flies in which CaLexA system was expressed (RNAi Off), (A"-E") show the fat body cells of flies in which the corresponding RNAi construct and CaLexA system was activated (RNAi On). Scale bar in A' represents $50 \mu \mathrm{m}$. All micrographs shown were taken with the same magnification. This figure was prepared in cooperation with Dr. J. Baumbach.

G. Quantification of the membrane GFP signals in flies containing the indicated RNAi transgenes. Relative GFP fluorescence of fat body tissues was normalized to the value of RNAi Off flies. RNAi Off refers to the controls ( $\left.A^{\prime}-E^{\prime}\right)$, RNAi On refers to flies in which the corresponding RNAi transgene was activated, (A"-E"). Note that the fat body cells showed reduced GFP signal level in response to RNAi expression in fat body cells. Error bars represent the Standard Deviation of samples. ${ }^{*}=p<0.05,{ }^{* *} ;=p<0.01,{ }^{* * *}=p<0.001$ (Student's t-test).The figure is based on Baumbach J., and Xu, Y., et al. JGG. 2014. This Figure was kindly provided by Dr. J. Baumbach. For details of the genetic constitutions of flies and experiments see 2.1.1, 2.1.2, 2.1.3, 2.4.3.

\subsection{Identification and characterization of Long-term Impairment of STIM function induced Obesity (LISO) in adult flies}

Long-term knockdown of Stim by six days of RNAi expression was employed to study the underlying mechanisms of fat storage (Baumbach, 2014; Baumbach et al., 2014a). However, the long-term knockdown by six days of incubation at $29^{\circ} \mathrm{C}$ is stressful for flies (Sayeed and Benzer, 1996). To minimize the interference of the high temperature incubation with fat storage, the incubation time was reduced from six days to a transient incubation period of 34-38 hours of the adult flies. Transient $29^{\circ} \mathrm{C}$ incubation treatment revealed that flies continued gaining fat and becoming obese even one month after the treatment (Baumbach, 2014; Borcherding, 2014). Although flies got obese after a long-term knockdown of Stim for six days, obesity resulting from Transient $29^{\circ} \mathrm{C}$ incubation offered the chance to study how Drosophila develops without the long-term temperature shift interference in response to $\mathrm{TI}$.

In the following, I will refer to the $38 \mathrm{~h}$ RNAi expression by temperature change as Transient temperature-shift Induced RNAi expression (TtI), which allows me to examine the persisting, long-term effects of transient Stim impairment by RNAi on body fat accumulation. In order to avoid a possible interference of the high temperature, I induced RNAi transgene expression in response to the drug mifepristone to which I refer to as Transient drug-feeding Induced RNAi expression 
(TdI) and asked whether this type of RNAi induction causes persistent, long-term fat accumulation in the treated flies as well.

\subsubsection{Stim-Transient temperature-shift Induced RNAi expression (Stim-Ttl) in} adult Drosophila males cause a long-term functional impairment of STIM and promotes long-term fat storage increase

According to features of the TARGET system (McGuire, 2003), the short-term $29^{\circ} \mathrm{C}$ incubation in adult flies should provoke Transient temperature-shift Induced RNAi transgene expression and result in a targeted gene knock down in fly fat body tissue (Ttl On), while no RNAi is switched on in the in the same temperature-inducible RNAi transgene flies kept at $18^{\circ} \mathrm{C}$ (Ttl Off). As controls, I used also flies which carry a temperature non-inducible RNAi transgene as "negative control flies", which received the transient $29^{\circ} \mathrm{C}$ treatment ( $\mathrm{TtI}$ On negative control), and the same flies without heat treatment (always kept at $18^{\circ} \mathrm{C}$ ) as Ttl Off negative control (Figure 5A, B). The time period when flies were treated with Ttl On, Ttl On negative control, or without treatment $\mathrm{Ttl}$ Off, $\mathrm{Ttl}$ Off negative control respectively was in short term as Ttl, for example Stim-Ttl. After Stim-Ttl, flies with respective treatment were called as Stim-Ttl On flies, Stim-Ttl Off flies, Stim-Ttl On negative control flies, or Stim-TtI Off negative control flies.

To confirm that the fat body-specific TARGET system can be tightly switched On/Off, I examined the expression of primary dsRNA from Stim RNAi1 transgene in adult male fly abdomen by RT-qPCR. As shown in Figure 5C, the expression of primary dsRNA from the Stim RNAi1 transgene construct was effectively switched on. At day 0, Stim-Ttl On flies displayed a 30 fold higher dsRNA mRNA level than Stim-Ttl Off flies, and its expression was also effectively switched off at day 1, i.e. Stim-TtI On flies showed the same level of dsRNA as Stim-Ttl Off. The same result was obtained with the corresponding flies at day 10 after Stim-Ttl On/Off (Stim RNAi1). Previous RT-qPCR analysis and western blot data on fly abdomen indicated that the expression of Stim mRNA and protein returned to normal levels at day 5 after RNAi induction in Stim-Ttl On/Off flies (Baumbach, 2014). Since Stim is ubiquitously expressed and found in all tissues (Attrill et al., 2016), I specifically dissected abdomen fat body tissues from adult male flies for RT-qPCR analysis. Surprisingly, my RT-qPCR data revealed that the expression level of Stim remained decreased to about half of the level of Stim-Ttl Off flies at day 10 after Stim-Ttl On (Figure 5D). These findings show that there was a long-term functional impairment of Stim expression in adult male fly fat body tissues after Stim-TtI On. 

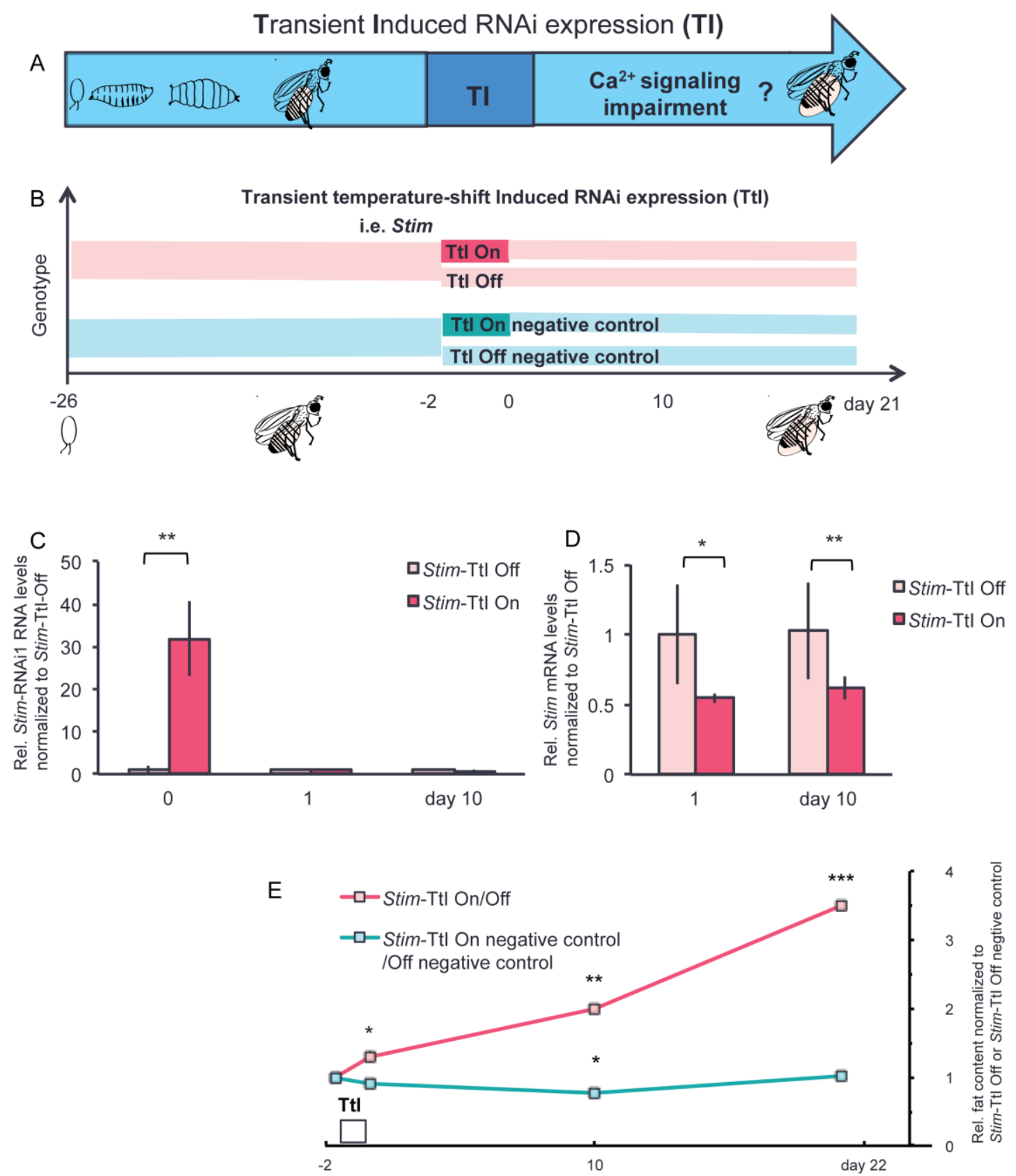

Figure 5. Stim-Transient temperature-shift induced RNAi expression (Ttl) in fat storage tissues of Drosophila adult males causes a long-term impairment of STIM function and promotes long-term body fat storage increase

A. Developmental scheme shows the time point of "Transient Induced RNAi expression" (TI) on calcium signalling genes in adult flies, e.g. TI on Stim, the key player of store-operated calcium entry (SOCE) at the day 6 after eclosion of adult male flies, which causes a long-term impairment of calcium signalling that leads to obese flies.

B. Developmental scheme shows the time period of either a temperature-shift (from $18^{\circ} \mathrm{C}$ to $29^{\circ} \mathrm{C}$; 38 hours (h) started at 6 days old adult male flies) or continuously keeping flies at $18^{\circ} \mathrm{C}$, which causes either Transient temperature-shift Induced RNAi expression (TtI) on a given gene e.g. Stim or not in flies containing temperature-inducible and fat storage tissue specific RNAi or temperature noninducible RNAi flies. 
"Ttl On" refers to Ttl activity in response to transient ( $38 \mathrm{~h}$ ) incubation of the flies at $29^{\circ} \mathrm{C}$ (red bar). The red bar indicates the time period when the Transient temperature-shift Induced RNAi expression was induced (Ttl On), e.g., Stim-TtI On. Flies that experienced Stim-TtI On were called as Stim-Ttl On flies in the following days after Stim-Ttl. "Ttl Off" refers to same temperature-inducible and fat storage tissue specific RNAi flies who were constantly kept at $18^{\circ} \mathrm{C}$ so that no $\mathrm{Ttl}$ activity was induced (light red bar), e.g., Stim-Ttl Off. Flies who always remained Stim-Ttl Off state were called as Stim-Ttl Off flies in the following days after transient $29^{\circ} \mathrm{C}$ incubation. .

"Ttl On negative control" refers to flies containing a temperature non-inducible RNAi transgene. These flies were also transiently kept at $29^{\circ} \mathrm{C}$ for $38 \mathrm{~h}$ (deep green bar), e.g., Stim-Ttl On negative control. Stim-temperature non-inducible RNAi flies who experienced transient $29^{\circ} \mathrm{C}$ incubation were called as Stim-Ttl On negative control flies after Stim-TtI On negative control.

"Ttl Off negative control" refers to flies of the same genetic constitution as the "TI On negative control" flies which, however, were constantly kept at $18^{\circ} \mathrm{C}$ (light green), e.g., Stim-Ttl On negative control. Stim-temperature non-inducible RNAi flies who were kept at $18^{\circ} \mathrm{C}$ were called as Stim-Ttl On negative control flies after Stim-Ttl On negative control.

In general, the time period when flies experienced either Ttl On, or Ttl Off, or Ttl On negative control, or Ttl Off negative control was called as Ttl, for e.g., Stim-Ttl.

C.D. RT-qPCR analysis was carried out on primary double stranded RNA (dsRNA) level from Stim RNAi1 transgene and mRNA expression level of Stim in Stim-Ttl On and Off adult male fly abdomen and fat body tissue, respectively. C. The primary dsRNA of Stim RNAi1 transgene was effectively over-expressed at day 0 after $38 \mathrm{~h}$ of $29^{\circ} \mathrm{C}$ heat induction of StimTtI ON (deep red) as compared to corresponding control flies (light red). Note also that the level of Stim RNAi dsRNA was reduced at day 1 and das 10 thereafter. D. The relative Stim mRNA expression level is significantly lower in adult male fly fat body tissue at day 1 , and day 10 after $38 \mathrm{~h}$ of Stim-Ttl On than in corresponding control (Stim-Ttl Off) adult male flies.

Relative (Rel.) dsRNA or mRNA levels are represented as fold change, which refers to the basis value $(=1)$ obtained from Stim-Ttl Off flies; Act5C mRNA served an internal standard and was used as reference to normalize the levels of mRNA expression. For details of experiment procedures of RT-qPCR see 2.2.4.1.

E. Quantification of the body fat contents of adult male flies at day 0, day 10 and day 21 after $38 \mathrm{~h}$ induction of Stim-Stim-Ttl On or Stim-Ttl On negative control as compared to Stim-Ttl Off or Stim-Ttl Off negative control flies. Note the long-term increased body fat storage. Relative (rel.) fat content is represented as fold change: TAG equivalent content/protein content of Stim-Ttl On or Stim-Ttl On negative control normalized with respect to the TAG equivalent content/protein content of Stim-TI Off or Stim-TI Off negative control flies serving as the respective controls. The Stim RNAi1 transgene line was used in above studies. Error bars represent the standard deviation of samples. ${ }^{*}=p<0.05,{ }^{* *} ;=p<0.01,{ }^{* * *}=p<0.001$ (Student's t-test). For details of the underlying genetic constitution of the flies see 2.1.1, 2.1.2, 2.1.5, 2.3.1, 2.3.3.

How does the Stim RNAi1 expression affect the Stim mRNA expression level in fat body tissue of Stim-Ttl On adult flies? Figure 5D shows that it was reduced to about $50 \%$ of normal expression level at day 1 as had been reported earlier (Baumbach, 2014). Furthermore, Baumbach (2014) also showed by Western blot analysis that the 
Stim protein level was down-regulated in these flies. To find out how Stim expression levels change in Stim-Ttl On flies, we also quantified the mRNA expression level

In addition, I examined body fat content alterations of adult male flies after Stim-TtI (Stim RNAi1). Consistent with the long-term decrease of Stim mRNA, the body fat content of Stim-TtI On flies was increased by $29 \%$ at day 1 after Stim-Ttl, and it was doubled at day 10 , and increased to a value that was about 3.5 fold higher than in Stim-Ttl Off flies at day 21(Figure 5E). In contrast, after Stim-Ttl, the body fat content of Stim-Ttl On negative control flies decreased to around $77 \%$ of body fat content level of Stim-Ttl Off negative control flies, but the body fat content difference between Stim-TtI On negative control and Stim-TtI Off negative control gradually diminished in the following 11 days (Figure 5E). Similarly, using and independent transgene Stim RNAi (Stim RNAi3) to administer Stim-TtI on 6 days old adult male flies, the body fat content of Stim-TtI On flies increased by around $36 \%$ at day 11 , and remained $21 \%$ higher than the value of Stim-Ttl Off flies at day 21 (Figure 23 (Supplementary data) A, B). On the other hand, the body fat content of Stim-Ttl On negative control (Stim RNAi 3) flies decreased to around $80 \%$ of body fat content level of Stim-Ttl Off negative control (Stim RNAi 3) flies at day 11, which maintain the around $30 \%$ less of body fat content of Stim-TtI Off negative control (Stim RNAi 3) flies at day 21 (Figure 23 (Supplementary data) A. B).

Taken together, these results provide evidence that Stim-Ttl On likely resulted in long-term functional impairment of Stim, which eventually lead to an continuous increase of body fat content after Ttl, although the temperature-stress of TtI normally induce the reduction of body fat content. 


\subsubsection{Stim-Transient drug-feeding Induced Stim RNAi expression (Stim-Tdl) in adult Drosophila males causes a long-term functional impairment of STIM and promotes long-term fat storage increase}

High temperature $\left(29^{\circ} \mathrm{C}\right)$ has been identified as kind of stress which substantially affects gene expression and the physiological performances of flies (Chen et al., 2015; Klepsatel et al., 2016). Thus, it might interfere with the primary effects of Stim RNAi expression. Therefore, to avoid possible interferences provoked by temperature differences, I established a drug inducible Stim-RNAi system, which is a combination of the GeneSwitch system (Roman et al., 2001) involving the druginducible (RU486; Mifepristone) inducible ubiquitously expressed Gal4 driver (daGS) line (Tricoire et al., 2009), and UAS-RNAi transgene lines.

Drug-inducible RNAi flies were grown to adult stage and then subdivided into two groups. One group was fed with food containing $200 \mu \mathrm{M}$ RU486 to cause Transient drug-feeding Induced RNAi expression On (termed TdI On), while the second group was fed with normal food to leave RNAi expression non-induced (referred to as Transient drug-feeding Induced RNAi expression Off (Tdl Off) (Figure 6A). In general, the time period when flies either experienced Tdl On, or Tdl Off, was called as Tdl, for example, Stim-Tdl. After Tdl, drug-inducible RNAi flies, which experienced Tdl On, were called Tdl On flies, for example, Stim-Tdl On flies; while genetically identical drug-inducible RNAi flies, which experienced Tdl Off (no drug exposure), were called Tdl Off flies, for example, Stim-Tdl Off flies (Figure 6A).

After drug food feeding, RT-qPCR on adult fly whole body showed that day 0 StimTdl On flies expressed around 10 fold higher Stim RNAi dsRNA than Stim-Tdl Off flies. Four days after Stim-Tdl On, Stim expression was reduced to the level of control flies (Stim-Tdl Off), and remained at a low level in both Stim-Tdl On and Off flies at day 8 (Figure 6B). Apparently, the drug-inducible Stim RNAi system has a weaker effect on dsRNA over-expression of Stim RNAi than the TARGET system. Nevertheless, the results show that it still can be reliably switched on and off. 
A
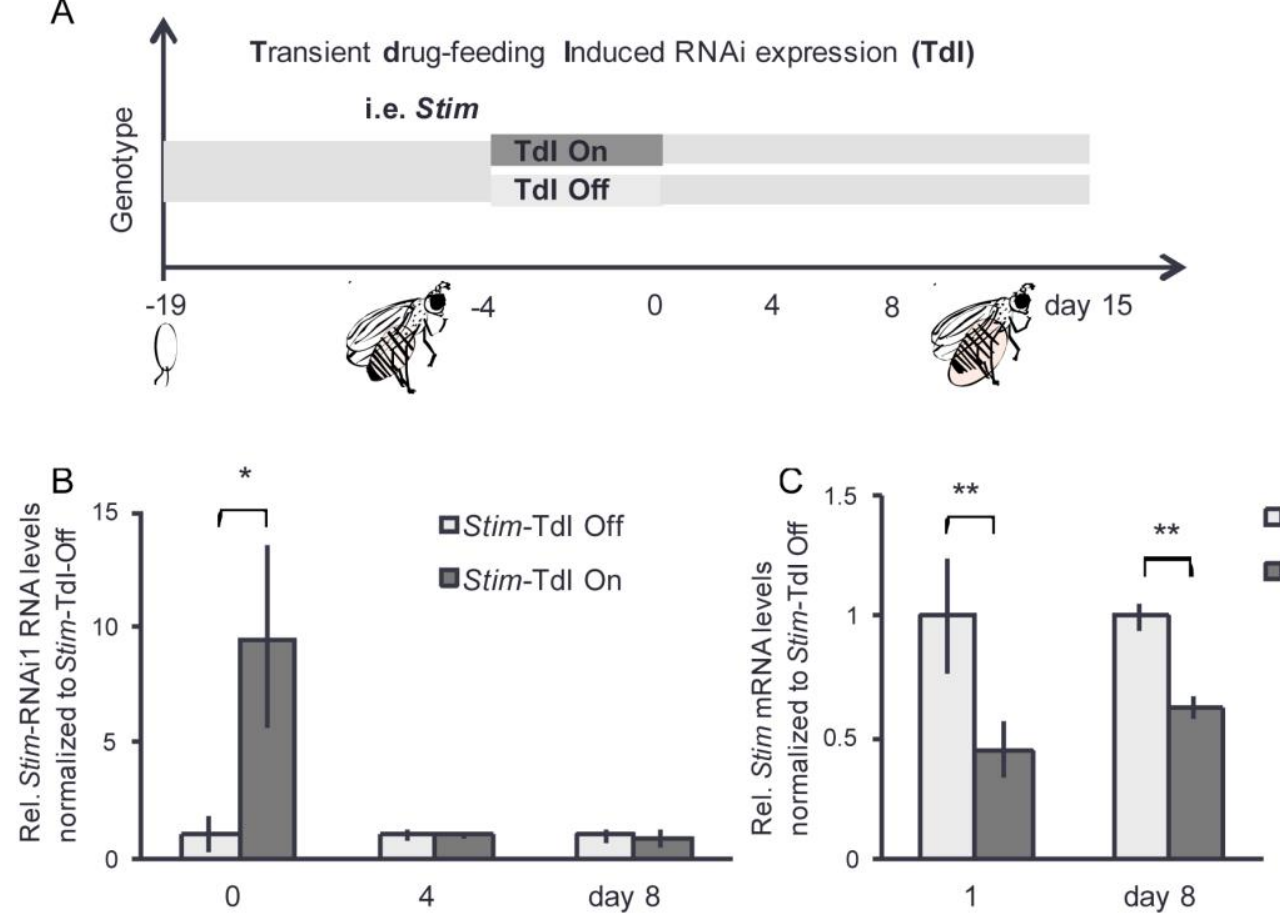

口Stim-Tdl Off

口Stim-Tdl On

D

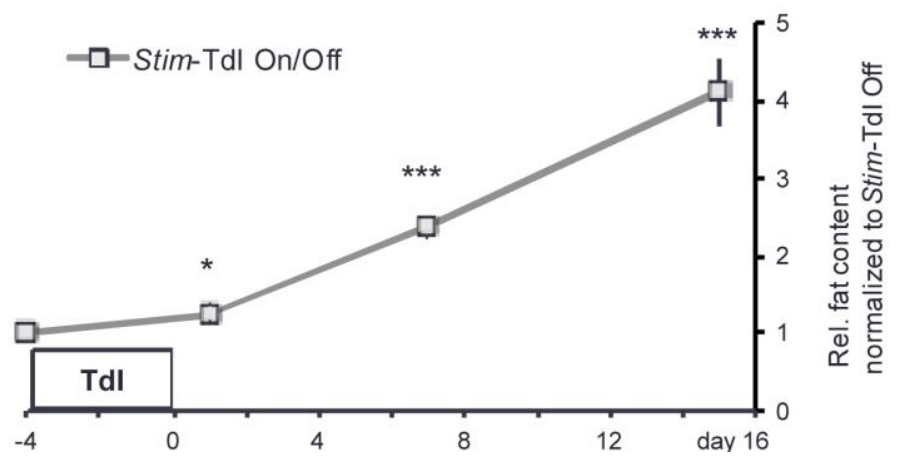

Figure 6. Stim-Transient drug-feeding induced RNAi expression (Tdl) in Drosophila adult males causes a long-term impairment of STIM function and promotes a long-term fat storage increase

A. Developmental scheme showing the time period of drug/control food-feeding (starting at 4 days old adult male flies, and ending 4 days later), which caused Transient drug-feeding Induced RNAi expression (Tdl) on a given gene such as Stim in flies containing a druginducible RNAi transgene or not. All the flies are raised at $25^{\circ} \mathrm{C}$.

"Tdl On" refers to the time period of drug-induction of RNAi expression $(200 \mu \mathrm{M}$ mifepristone that was present in Gö-food; dark grey bar) was switched on for 4 days, e.g. Stim-Tdl On. Drug-inducible RNAi flies, which experienced Tdl On were called Tdl On flies after Tdl, i.e., Stim-Tdl On flies. "Tdl Off " refers to drug inducible RNAi transgene containing flies, which were kept under non-induced conditions (4 days feeding with $200 \mu \mathrm{M}$ ethanol in Gö-food, light gray bar). Drug-inducible RNAi flies, which experienced Tdl Off were called Tdl Off flies after Tdl, e.g. Stim-Tdl On flies. In general, the time period when flies either experienced Tdl On, or Tdl Off, was called as Tdl, for example, Stim-Tdl.

B.C. RT-qPCR analysis was carried out on primary double stranded RNA level from Stim RNAi1 transgene and mRNA expression level of Stim in Stim-Tdl On and Off adult male whole flies. B. Result shows that the primary dsRNA of Stim RNAi1 was efficiently overexpressed at day 0 after the 4 days Stim-Tdl On in adult males, and was decreased to control levels at days 4 and 8 as compared to corresponding control (Stim-Tdl was not 
induced, Stim-Tdl Off) flies. (C) And the Stim mRNA expression level was reduced in adult male fat body tissue at day 1 and day 10 after Stim-Tdl On as compared to corresponding control (Stim-Tdl Off) flies. Relative (Rel.) mRNA levels are represented as fold change, which refers to the basis value $(=1)$ obtained from Stim-Tdl Off; Act5C mRNA served an internal standard and was used as reference to normalize the levels of above mentioned gene mRNA expression.

D. Quantification of fly body fat contents at day 1, 7, and 15 after 4 days Stim-Transient drugfeeding induced RNAi expression (Stim-Tdl On) as compared to corresponding control (StimTdl Off) flies showed long-term increased body fat storage. Relative (rel.) fat content is represented as fold change: TAG equivalent content/protein content of Stim-Tdl On flies normalized with respect to the TAG equivalent content/protein content of Stim-Tdl Off flies.

Stim RNAi1 transgene line was used in above studies. For details of the underlying genetic constitution of the flies and experimental conditions see 2.1.1, 2.1.2, 2.1.5, 2.3.1 and 2.3.3. Error bars represent the standard deviation of samples. ${ }^{*}=p<0.05,{ }^{* *} ;=p<0.01,{ }^{* * *}=$ $\mathrm{p}<0.001$ (Student's t-test).

Moreover, the RT-qPCR results also showed that Stim expression in Stim-Tdl On flies was reduced to about $50 \%$ when compared to the expression level in control (Stim-Tdl Off) flies at day 1, and that it remained at the low level at day 8 in Stim-Tdl On flies (Figure 6C). This means that the long-term impairment of Stim expression was further confirmed. Accordingly, the long-term functional impairment of STIM from Stim-Tdl On also continually promotes fly body fat content, which results in an about 4 fold higher fat content in those flies in day 15 flies as compared to Stim-Tdl Off control flies (Figure 6D).

In summary, the results obtained with the drug-inducible Stim-RNAi system provide compelling evidence that transient RNAi induction by both Stim-TtI On and Stim-Tdl On results in long-term functional impairment of STIM, which causes a long-term increase of the body fat content of flies.

\subsubsection{Stim-Ttl causes a long-term functional impairment of STIM and promotes long-term fat storage increase in adult flies except in mated females}

I next asked whether differences in age, sex or the reproductive state would affect the fat accumulation due to Stim- TtI. In previous studies, 34h Stim-TtI on

18 days old adult male flies were performed, and results show that 10 days later, flies stored significantly more fat than the control flies (Baumbach, 2014). Hence, I used Stim-Ttl On treatment on older flies (36 days old adult unmated male flies) (Figure 7 E). These males also showed an increased fat accumulation on day 21 after Stim RNAi expression (Stim-TtI On) (Figure 7 E) as had been observed earlier with the 6 days old males (Figure 7A). 

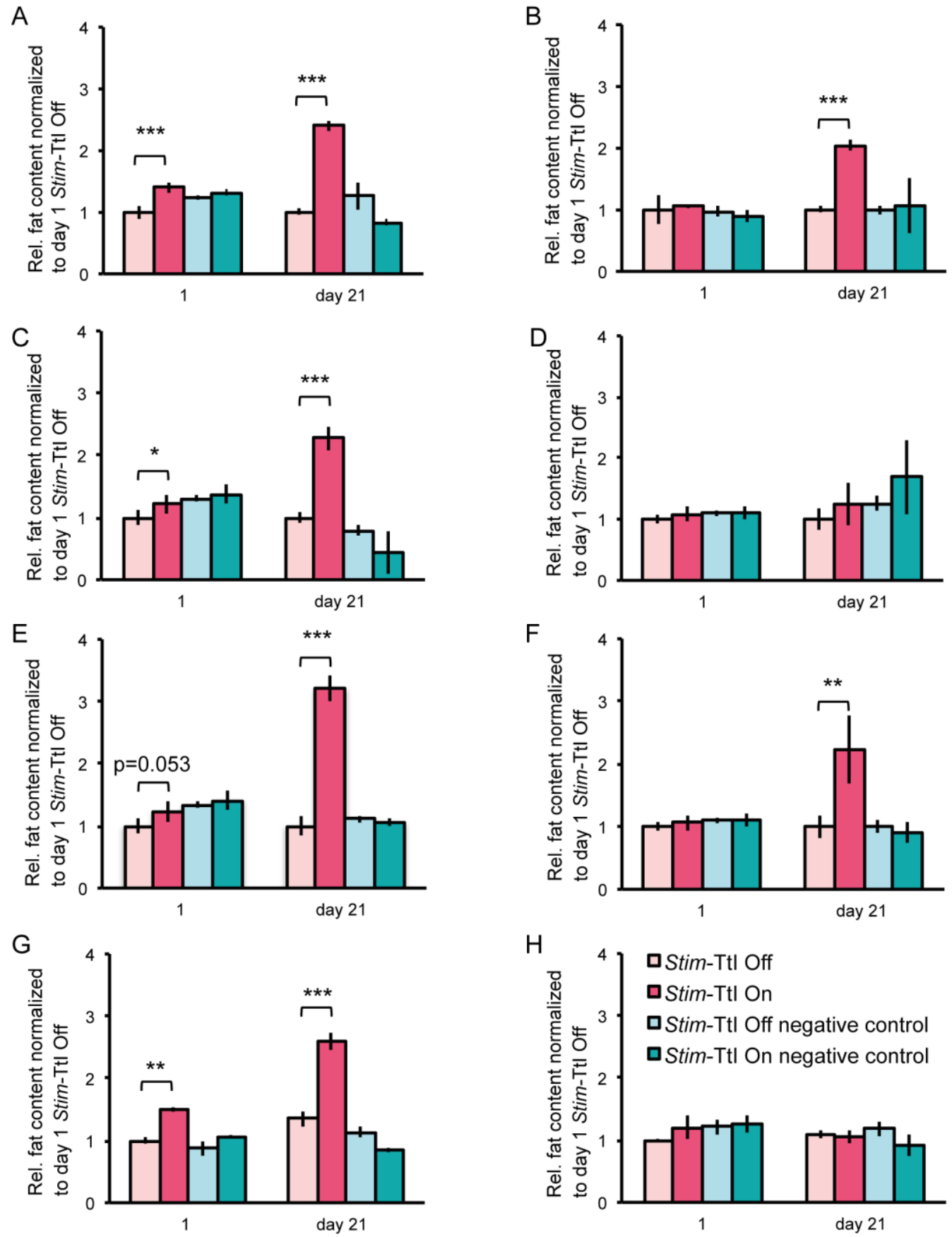

Figure 7. Stim-Ttl promotes long-term fat storage increase in adult flies except in mated females

A-H. Quantification of the body fat contents on day 1 and days 21 after Stim-Transient temperature-shift Induced RNAi expression for $38 \mathrm{~h}$ (Stim-TtI On) in adult male and female flies, and on corresponding control (Stim-Ttl Off, Stim-Ttl On negative control, Stim-Ttl Off negative control) flies. Stim RNAi1 transgene line was used in these studies. Relative (rel.) fat content is calculated as fold changes: TAG equivalent content/protein content of Stim-TtI On/Off or Stim-TtI On negative control flies normalized with respect to the TAG equivalent content/protein content of day $1 \mathrm{Stim}$-TI Off flies (standard value). For details on the nomenclature of Ttl On, Ttl Off, Ttl On negative control, Ttl Off negative control see Figure 3. Stim-Ttl On causes long-term increased body fat storage in 6 days old non-mated adult male 
flies (A), six days old virgin female flies (B), 36 days old non-mated adult male flies (E), 36 days old virgin female flies (F), and 36 days old mated male flies (G), but not in six (D) and 36 days old mated female flies $(\mathbf{H})$. For details of the underlying genetic constitution of the flies and the experiments procedures see 2.1.1, 2.1.2, 2.1.4, 2.3.1 and 2.3.3. Error bars represent the standard deviation of samples. ${ }^{*}=p<0.05,{ }^{* *} ;=p<0.01,{ }^{* * *}=p<0.001$ (Student's t-test).

Additionally, Stim-Ttl On was administered on 6 days (Figure 7B), and 36 days (Figure 7F) old adult virgin female flies, respectively. In these flies, Stim-TtI On also promoted fat storage of virgin female flies, irrespective of their age (Figure 7B, F). These results indicate that the aging process does not mitigate the excessive fat storage due to the earlier Stim RNAi treatment.

To test whether reproduction plays a role in the increase of fat content after Stim-Ttl, the same number of adult male and female flies was kept after eclosion in one vial and they were treated with Stim-TtI On/Off, after 6 days and 36 days, respectively. Figure $7 \mathrm{C}$ and $\mathbf{G}$ show that mating does not prevent 6 and 36 days old male flies from accumulating more fat after Stim-Ttl On. In contrast, no such effect could be observed with 6 and 36 days old mated female flies treated with Stim-Ttl On (Figure 7D, H). In such females, no significant fat content increase their fat contents could be observed (Figure 7D, H). This finding suggests that reproduction process (e.g. oogenesis) might consume fat stored in female flies, which eliminates the fat increase that could result from Stim-TtI On.

In summary, these data indicate that fat increase in response to a long-term functional impairment of STIM is a general phenomenon in flies with the exception of females that are in the reproduction stage.

\subsubsection{Ttl of candidate gene $A k h R$ which acts upstream of Stim promotes also an increase of fat storage}

There is evidence that supports the hypothesis that Gaq49b, Gy1, Plc21C, and STIM may transduce Akh/AkhR signalling to increase the $\mathrm{iCa}^{2+}$, level which result in the mobilization of the neutral lipid stores (Figure 3 and 4) (Baumbach et al., 2014b). If these molecules work in the same pathway, one can expect that Ttl of the respective SOCE putative upstream genes also cause long-term abnormal accumulation of fat, which has been indicated by the later fat storage increase after knockdown of Gaq49b, Gy1, Plc21C, and STIM gene (Borcherding, 2014). Thus, I performed TtI studies on 6 days old adult male flies using RNAi directed against the above 
mentioned genes proposed to act upstream of Stim, and examined the body fat content of the respective flies after 11 and 21 days. The results in Figure 8A show that after $A k h R-\mathrm{Tl}$ On treatment (using a long hairpin RNA AkhR RNAi transgene) caused a 1.76 -fold increase of body fat in 21 days old males (Figure 8A).

A

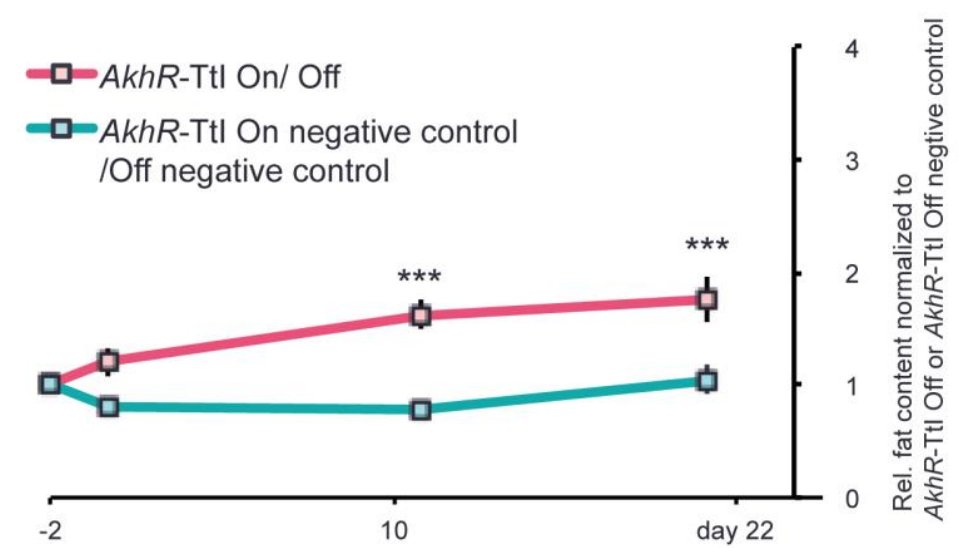

Figure 8. Ttl of Stim upstream candidate gene-AkhR promotes long-term fat storage increase

A. Quantification of the body fat contents on day 0 or 1, 11 and 21 adult male flies after Ttl of Stim upstream candidate genes for 48 hours. AkhR RNAi1 transgenes lines were used in following studies respectively. Relative (rel.) fat content is calculated as fold changes: TAG equivalent content/protein content of $A k h R$-Ttl On or $A k h R$-Ttl On negative control normalized with respect to TAG equivalent content/protein content of day 0 AkhR-Ttl Off flies. For details on the nomenclature of Ttl On, Ttl Off, Ttl On negative control, Ttl Off negative control see Figure 3. Reduction of $A k h R$ expression by $A k h R$-Ttl On for 48 hours promotes body fat storage increase at day 11 and 21 after the induction time point. For details of the underlying genetic constitution of the flies and the experiments procedures see 2.1.1, 2.1.2, 2.1.4, 2.3.1 and 2.3.3. Error bars represent the standard deviation of samples. ${ }^{*}=p<0.05,{ }^{* *} ;=p<0.01$, ${ }^{* * *}=p<0.001$ (Student's t-test).

On the basis of the results above, one can conclude that it is possible the long-term impairment of SOCE and intracellular $\mathrm{iCa}^{2+}$, resulting from the transient induction of Stim upstream genes $A k h R$, which leads to the enhanced fat accumulation in adult flies.

\subsubsection{Long term Impairment of STIM function induced Obesity (LISO)}

Abnormal fat accumulation has been defined as the feature of obesity in humans (Garrow, 1988; WHO Consultation on Obesity, 2000). In order to test whether the body fat accumulation is indeed due to calcium signalling dysfunction, I focused the analysis on the phenotypes, which are caused by Stim- Ttl in 6 days old adult male flies. 
In healthy humans, fat is mainly stored in white adipose tissues, which is composed of different depots such as the visceral adipose tissues and subcutaneous adipose tissue. Visceral adipose tissue is centrally located, and surrounds other internal organs such as stomach, liver, intestine, pancreas or kidney, while subcutaneous adipose tissue is located directly below the skin for example, the abdominal, gluteal subcutaneous or thigh adipose tissue (Bernardo Le'o Wajchenberg, 2000; Bjørndal et al., 2011; Thomas et al., 2013). Android fat, especially the fat accumulation in visceral adipose tissue, has been associated with increased risks of developing severe diseases including the metabolic syndrome (Bi et al., 2014b; Després and Lemieux, 2006; Jensen, 2008; Kang et al., 2011; Patel and Abate, 2013; Walker et al., 2014), cardiovascular diseases (Britton et al., 2013; Després, 2012; Lim and Meigs, 2013; Neeland et al., 2015), cancer (Britton et al., 2013), polycystic ovary syndrome in women (Cosar et al., 2008), as well as sleep disorder (Bhatia et al., 2015). Recently, waist circumference (WC) emerged as a convenient and quantitative parameter for android adiposity, which has been strongly associated with metabolic syndrome (Smith, 2015) and mortality risks (Petursson et al., 2011; Pischon et al., 2008; Seidell, 2010; Simpson et al., 2007; World Health Organization, 2008).

To explore whether excessive fat accumulation due to Stim-Ttl can serve as a model for human obesity research, I decided to characterize the abdominal fat accumulation of flies in details. Due to practical considerations, such as an interference in reproductive or ageing flies, I focused on 6 days old adult male flies together with sex- and age-matched controls to test the effects of Stim-Ttl in the following studies such as systematically profiling their fat storage, the physiological and behavioral differences in order to explore the underlying regulation mechanisms at these different levels.

In order to directly assess the site of fat storage in flies, i.e. their abdomen, I removed their legs and wings, and examined the bodies of day 1 and day 21 Stim-TtI On and Off adult male flies under the microscope. As shown in Figure 9A, no shape difference could be found between day 1 Stim-Ttl On and day 1 Stim-Ttl Off flies. However, the abdomina of day 21 Stim-Ttl On flies were swollen and pale as compared to the corresponding Stim-Ttl Off flies (Figure 9B). This shows that StimTtI On flies can eventually develop a "big belly" similar to obese individuals. 
I also examined dissected abdomen without intestines and reproduction organs. There was no obvious morphology difference of abdominal fat body tissues (analogous to human adipose tissue and liver) between day 1 Stim-TtI On and Off flies (Figure 9A', A'). While, more expanded and crowded fat tissue could be found in day 21 Stim-Ttl On flies than day 21 Stim-Ttl Off flies (Figure 9B', B'”). In accordance with abdomen and abdominal fat tissue images on day 21 of Stim-TtI On, the flies were about $18 \%$ heavier than that the day 21 Stim-Ttl Off flies, and about $22 \%$ heavier than day 1 Stim-TtI Off flies (Figure 9C).
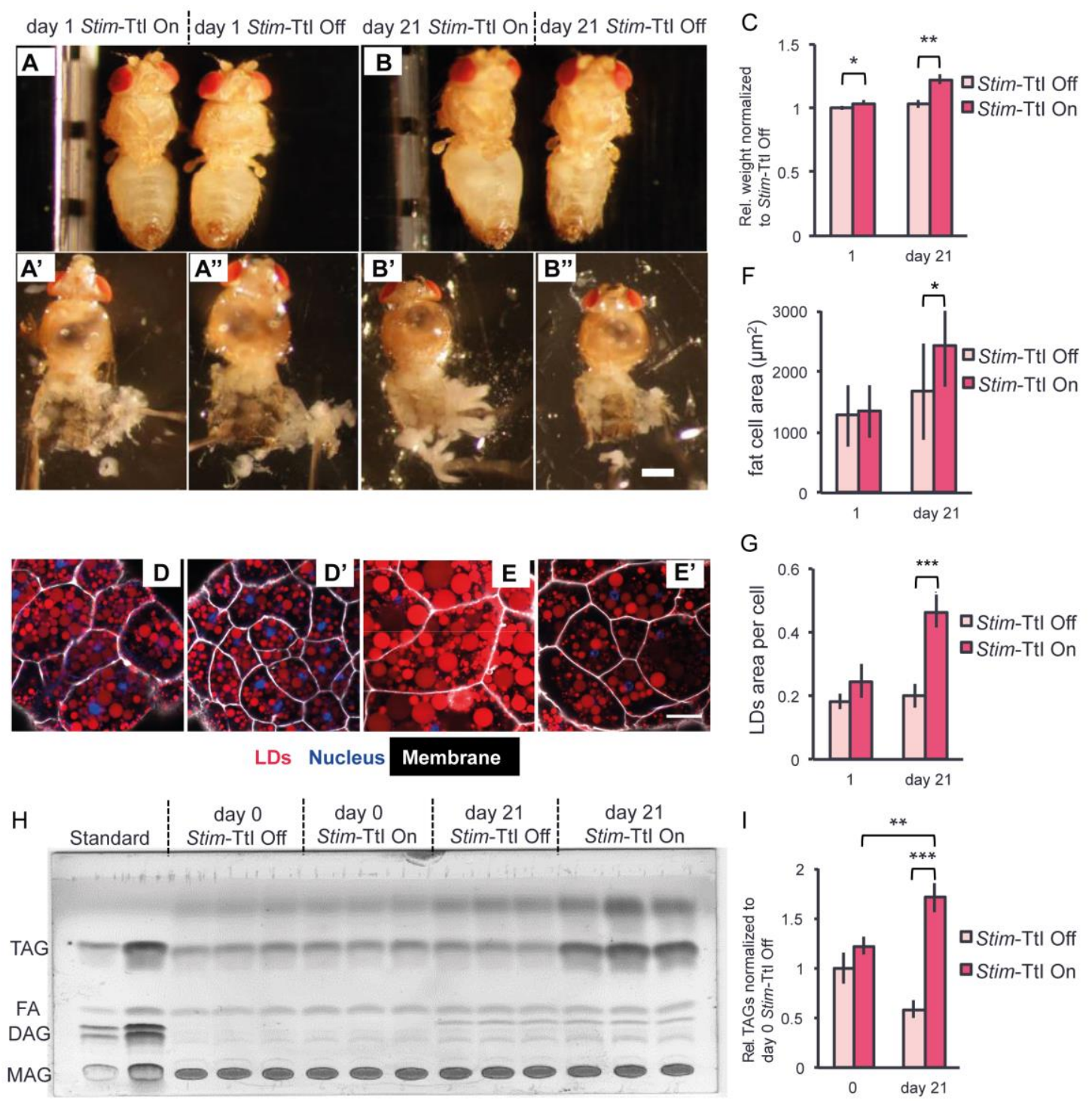

Figure 9. Stim-Ttl in fat storage tissue of Drosophila adult males causes long-term Impairment of STIM function induced Obesity (LISO)

Microscopic images of day 1 (A) and day 21 (B) after Stim-RNAi expression (Stim-TtI On, red); and the corresponding control flies (Stim-Ttl On, light red). Note that wings and legs have been removed to better visualize the swollen abdomen at day 21 of Stim-Ttl On adult male flies. Vividissected flies (open abdomens to visualize the fat tissue) show similar morphology of fat body tissue at day 1 of Stim-Ttl On (A') as compared to the morphology of 
corresponding Stim-Ttl Off (A") adult male flies. Note that fat body tissues increased at day 21 of Stim-Ttl On adult males (B') as compared to the corresponding control male (Stim-Ttl Off) (B') flies. Scale bar in B" represents $0.5 \mathrm{~mm}$. All micrographs (A-B') were taken with the same magnification. C. Body weight measurements showing that adult male flies have increased wet body weight at day 21 of Stim-Ttl On adult males as compared to the corresponding control male (Stim-Ttl Off) flies (For experiment details see 2.5.1).

D. Confocal microscopy ex vivo images show lipid droplets (stained by LD540; red), cell plasma membranes (stained by CellMask Deep red; white) and nuclei (stained by DAPI; blue) in abdominal fat body tissue of adult male flies at day 1 (D) and day 21 of Stim-TtI On (E) and the corresponding control male (Stim-Ttl Off) flies at day 1 (D') and day 21 (E'). Scale bar in E' represents $25 \mu \mathrm{m}$. All micrographs (D-E') were taken with the same magnification.

F. Fat cell area quantification based Cell mask stained membrane signals show an increased size of fat body cells at day 21 of Stim-Ttl On in adult males as compared to the corresponding control male (Stim-TtI Off) flies. G. Quantification of the LD540 stained lipid droplet (LD) area in each fat body cell indicates an increased fat storage at day 21 of Stim-TI On in adult males as compared to the corresponding control male (Stim-Ttl Off) flies. For details of microscopy and confocal imaging experiment procedures see 2.4.1, 2.4.2.

H. Thin layer chromatography (TLC) shows major neutral lipid classes at day 1 and day 21 of Stim-Ttl On and the corresponding control male (Stim-Ttl Off) flies, respectively. MAG: Monoacylglycerides; DAG: Diacylglycerides; FA: Fatty acids; TAG: Triacylglycerides. Standard refers to the different concentrations of mixture of MAGs, DAGs, FAs and TAGs to identify the relevant lipid classes. I. Integrated intensity quantification of the TAG bands (see $\mathrm{H}$ ) indicates an increased TAG content at day 21 of Stim-TtI On in adult males as compared to the corresponding control male (Stim-Ttl Off) flies. For details of TLC experiment procedures see 2.3.5. Stim RNAi1 transgene line was used in above studies. Error bars represent the standard deviation of samples. ${ }^{*}=p<0.05,{ }^{* *} ;=p<0.01,{ }^{* * *}=p<0.001$ (Student's t-test). For details on the nomenclature of Stim-Ttl On, Stim-Ttl Off, Stim-Ttl On negative control, Stim-Ttl Off negative control see Figure 3. First round experiments in this figure were kindly done by the Bachelor student Annika Franziska Borcherding (Borcherding, 2014).

To better view and document how post Stim-TtI On flies accumulate their extra fats at cellular level, I employed LD540, DAPI, and CellMask ex vivo staining and used microscopic techniques to display the storage patterns of lipid droplets (LD) in fat body cells of male flies. It has previously been shown that long-term (6 days) and short-term induction of Stim-RNAi expression resulted in an elevated fat storage as compared to the tissues from corresponding control flies (Baumbach et al., 2014a; Borcherding, 2014). However, how fat cell size and cell LDs area quantitatively change during obesity development after Stim-RNAi was not yet established. Therefore, I quantified the fat cell and LDs imaging data of day 1 and day 21 Stim-TtI On/Off flies. Fat cell size did not appear to increase in day $1 \mathrm{Stim}$-Ttl On flies (Figure 9D, D', F), while the average cell area size of day 21 Stim-TtI On flies increased by around $46 \%$ as compared to the day 21 Stim-Ttl Off flies (Figure E, E', F). 
The LDs area per cell in Stim-Ttl On flies confirmed the body fat content data. It showed a modest increase as compared day 1 Stim-Ttl Off flies, albeit LDs area per cell differences was not statistically significant (Figure 9D, D', G). After 21 days, however, there was considerable increase with respect to the LDs area per cell in Stim-Ttl On flies, reaching an increase of about $46 \%$ of cell area, which was around 2.3 fold higher than that observed with day 21 Stim-Ttl Off flies (Figure 9E, E', G).

Moreover, thin layer chromatography (TLC) on day 1 and day 21 post Stim-TtI On/Off whole flies sorted out the different neutral lipid classes and fatty acids: Monoacylglycerides (MAG), Diacylglycerides (DAG), Fatty acids (FA) as well as Triacylglycerides (TAG) (Figure 9H). Among them, the amounts of TAGs was strikingly different between day $21 \mathrm{Stim}$-TtI On and Stim-TtI Off flies, i.e. day 21 StimTtl On flies contained about 1.9 fold more TAGs compared to day 21 Stim-Ttl Off flies, which is also around $71 \%$ higher than the values observed with day 0 Stim-Ttl Off flies (Figure 9H, I).

In summary, the long-term impairment of STIM function from Stim-TtI On caused an increase of the TAG storage, resulting in more LDs within larger fat cells, which gradually promotes the expansion of abdominal fat tissue and the increase of whole fly body weight. I refer to this phenomenon, which can serve as a Drosophila obesity model, as Long-term Impairment of STIM function induced Obesity (LISO). In the following, I call LISO in response to Stim-Transient temperature-shift Induced RNAi (TtI) as t-LISO, while I refer to LISO caused by Stim-Transient drug-feeding Induced RNAi (TdI) as d-LISO.

\subsubsection{LISO affects the physiological performances of flies}

To confirm that LISO can serve as model for human obesity (Garrow, 1988; WHO Consultation on Obesity, 2000), I next asked whether LISO also poses risks to the health, i.e. the physiological performance of flies.

Obese flies have been found to be more resistant to food deprivation (Grönke et al., 2005, 2007; Liu et al., 2012; Subramanian et al., 2013). Thus, neutral lipid mobilization and starvation resistance of day 21 Stim-TtI On/Off and negative control flies were assessed under acute starvation conditions. 

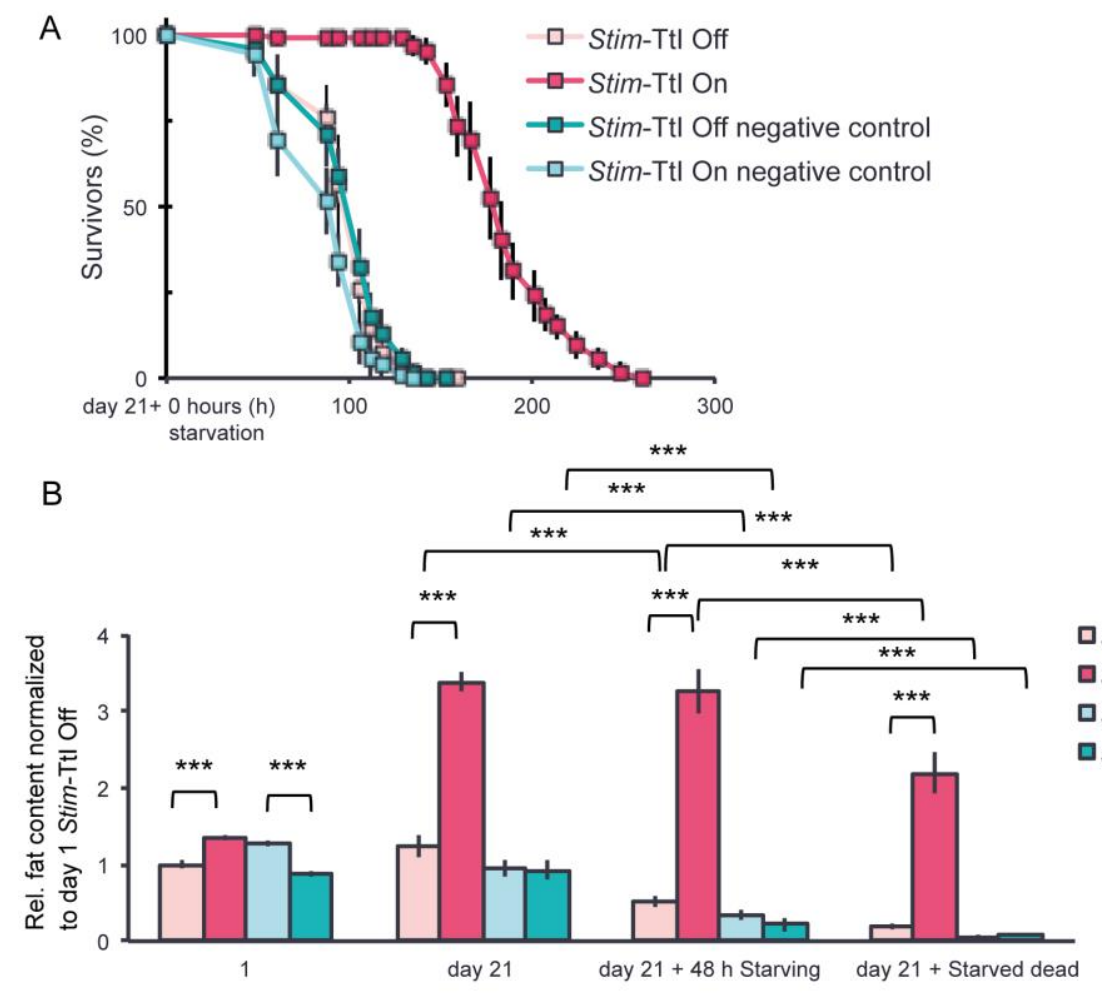

口Stim-Ttl Off

口Stim-Ttl On

口Stim-Ttl Off negative control

口Stim-Ttl On negative control

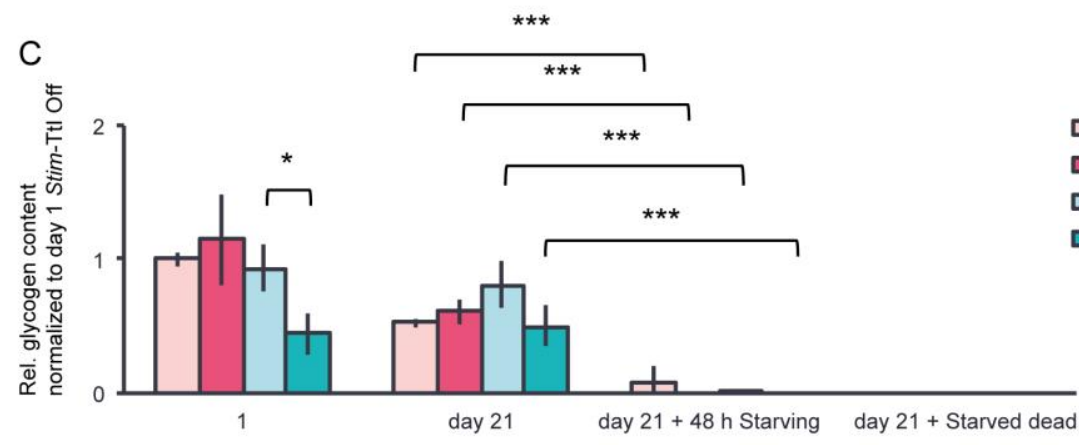

口Stim-TtI Off

口Stim-Ttl On

Stim-Ttl Off negative control

口Stim-Ttl On negative control

D

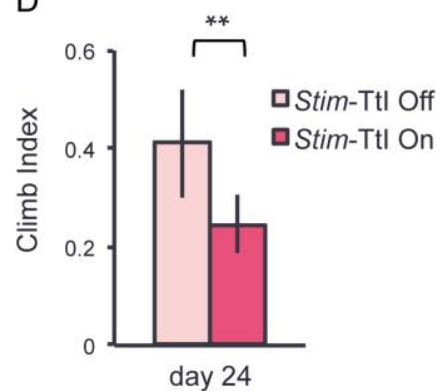

E

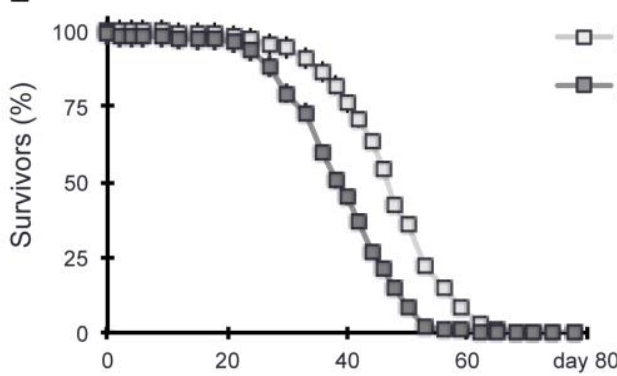

Figure 10. LISO affects the physiological performances of flies

A. Starvation resistance of day 21 adult male flies: Stim-Ttl On flies (red); and correspondingly treated control flies: Stim-Ttl Off flies (light red); or Stim-Ttl On negative control flies (deep green), Stim-Ttl Off negative control flies (light green). (Stim-TI Off, n=271; Stim-TI On $\mathrm{n}=268$, Stim-TI Off negative control, $\mathrm{n}=263$ and Stim-TI On negative control, $\mathrm{n}=342$; for details of the nomenclature see legend of Figure 3. Note that the Stim-Ttl On significantly extends the ability of the flies to survive in the absence of food (starvation). StimTtl On / Stim-Ttl Off: $p<0.0001$; Stim-Ttl On negative control / Stim-Ttl Off negative control: $p<0.0001$ (log rank test). 
B. C Quantification of the body fat contents and the body glycogen contents of adult male Stim-Ttl On flies (red)) and control males (Stim-Ttl Off flies (light red), Stim-Ttl On negative control flies (deep green), Stim-Ttl Off negative control flies (light green)) at day 1 and day 21, at day 21 followed by starvation for 48 hours ("21 days + $48 \mathrm{~h}$ Starving") as well as at day 21 followed by starvation until all flies have died (21 days + Starved dead). B. Relative (rel.) fat content is represented as fold change: TAG equivalent content/protein content of Stim-TtI On/Off or Stim-TtI On/Off negative control adult male flies normalized with respect to the TAG equivalent content/protein content of day $1 \mathrm{Stim}$-Ttl Off adult male flies (standard value). Note the increased fat accumulation of day $21 \mathrm{Stim}$-TtI On adult male flies and their compromised lipid mobilization during the starvation (48 hours). Note also that the fat reserves are not completely mobilized in Stim-Ttl On flies that were starved to death. C. Relative (rel.) glycogen content is represented as fold change: glycogen content/protein content of Stim-TtI On/Off or Stim-Ttl On/Off negative control adult male flies normalized with respect to the glycogen content/protein content of day $1 \mathrm{Stim}$-Ttl Off adult male flies (standard value). Note that in contrast to fat content (B), the glycogen content of the males (C) was not affected by the transient Stim RNAi expression. Upon starvation for 48 hours and after starvation to death, respectively, flies are nearly and completely deprived of their glycogen stores. For details of glycogen assay see 2.3.4.

D. The reduced climbing index indicates that Stim-TtI On (red) affects the climbing ability of males after 24 days as compared to corresponding control flies (Stim-Ttl Off).

Error bars represent the standard deviation of samples. ${ }^{*}=p<0.05,{ }^{* *} ;=p<0.01,{ }^{* * *}=$ $p<0.001$ (Student's t-test). For details of climbing assay see 2.5.3.

E. 4 days of Stim-Tdl On reduces the lifespan of male flies as compared to corresponding control (Stim-Tdl was not induced, Stim-Tdl Off) flies. Error bars represent Standard Error of mean, $p<0.001$ (log rank test). For details on the nomenclature of Stim-TtI On, Stim-TtI Off, Stim-Ttl On negative control, Stim-Ttl Off negative control see Figure 3, For details on the nomenclature of Stim-Tdl On, Stim-Tdl Off see Figure 4. All experiments were performed with the Stim RNAi1 transgene line. For details of lifespan assay see 2.5.4.

LISO flies displayed substantially stronger starvation resistance as compared to any of the control flies, since they survived almost twice as long than Stim-Ttl Off flies (Figure 10A). Acute lipid mobilization of LISO flies was impaired. During the first 48 hours of starvation, almost no fat appeared to be consumed in Stim-Ttl On flies, while Stim-Ttl Off flies had consumed $61 \%$ of their fat storage (Figure 10B). After starving the flies to death, when lipids were almost completely depleted in Stim-TtI Off and Ttl On/Off negative control flies, the Stim-Ttl On flies died despite of excessive amount residue fat. They still contained about $45 \%$ of their pre-starvation fat levels (Figure 10B). Nevertheless, during the starvation process, Stim-TtI On flies consumed about 40\% more fat than Stim-Ttl Off flies (Figure 10B). In addition, since glycogen was found to be an energy reserve during starvation (Baumbach et al., 2014a; Choi et al., 2011), I also monitored the changing glycogen content during the starvation of the flies. In contrast to fat, the glycogen storage levels of fed and starved flies were similar between Stim-TtI On, Stim-Ttl and Stim-TtI On/Off negative 
control flies (Figure 10C). These results indicate that Stim-Ttl On flies have impaired abilities of flies to make use of their entire lipid storage during starvation, but they had normal glycogen mobilization capabilities.

Recent studies have shown that obesity is accompanied by physical inactivity of both flies (Birse et al., 2010) and mice (Friend et al., 2016). In flies, the ability to perform physical activities was monitored with negative geotaxis, i.e. the climbing ability from bottom to top in upright tubes, a capacity which declines with flies aging (Birse et al., 2010; Gargano et al., 2005). The climbing assay has been used to indicate the unhealthy state of fly neuromuscular system in diseases such as Parkinson, muscle dystrophy, and Alzheimer (Benzer, 1967; Chakraborty et al., 2011; Feany and Bender, 2000; Gáliková et al., 2015; Rees et al., 2009; Shcherbata et al., 2007). Given the excessive fat accumulation in LISO flies, I monitored their climbing abilities. As is shown in Figure 10D, LISO significantly undermined climbing ability by $40 \%$, which reveals the LISO's adverse effects on the fly's health.

Moreover, large-scale epidemiological studies document a link between obesity and higher mortality risk in humans (Flegal et al., 2013; Petursson et al., 2011; Pischon et al., 2008; World Health Organization, 2008). Also in flies it was found that obesity is associated with fly reduced lifespan (Grönke et al., 2005; Skorupa et al., 2008; Woodcock et al., 2015). To determine whether LISO affects longevity or not, I performed life span experiments with d-LISO (Stim-Tdl On) and its corresponding control (Stim-Tdl Off) flies. Similar to the findings with human obesity patients, the median lifespan of the LISO flies was indeed considerably shortened by about $21 \%$ (Figure 10E). The data therefore suggest that LISO exert detrimental effects on the lifespan of flies.

Overall, these results show that LISO-induced obesity affects the physiological performances of flies such as lipid mobilization, climbing abilities, lifespan, and that it is therefore a critical risk indicator for physical activity as well as unhealthy ageing in adult flies. The results also support the argument that LISO can function as an model for obesity and related diseases studies in human. 


\subsection{Dysfunctional energy homeostasis in response to a Long-term Impairment of STIM signalling causes LISO}

Like in humans, the excessive energy storage in form of TAG in temperature-shift or drug feeding induced LISO (t-LISO or d-LISO) flies is the result of energy imbalance. To address this assumption, I checked the energy intake as well as the energy expenditure of $\mathrm{t}$-LISO and d-LISO flies.

\subsubsection{Dysfunctional energy homeostasis after Stim-Ttl contributes to t-LISO}

Obesity has been attributed to excess food intake in human (Swinburn et al., 2009; Vandevijvere et al., 2015), mice (Coleman, 1978) as well as Drosophila (Al-Anzi et al., 2009; Baumbach et al., 2014a; Min et al., 2016). In Drosophila, the CAFE assay (Ja et al., 2007), which is based on providing single fly with liquid food via a capillary, was used to track the food intake of the flies. Stim-Ttl On flies showed a $90 \%$ increased food intake during Ttl similar as observed previously (Baumbach et al., 2014a) and consumed about 68\% more at day 1 after Ttl as compared to Stim-Ttl Off flies (Figure 11A). I also observed that TtI On negative control flies exert a $63 \%$ increase of food at Ttl, but this rise in food intake disappeared at $1^{\text {st }}$ day after Ttl when compared to Ttl Off negative control flies (Figure 11A). Stim-Ttl On flies maintained $50 \%$ higher food intake in following 10 days after Ttl (Figure 11B). These results support the conclusion that it is Stim-TtI On activity that contributes to hyperphagia of t-LISO flies rather than the temperature shift of TtI.

To determine whether the persistent hyperphagia contributes to adiposity of t-LISO, I examined the body fat content of Stim- Ttl On flies and compared it to the corresponding control flies providing either ad libitum feeding conditions, i.e no restriction on food amounts or so called pair-fed conditions, under which flies are provided with a fixed amount of food, which was slightly less than the food amount consumed by Stim-TtI Off flies. Under ad libitum conditions provided by the CAFE system (details described in 2.5.5), the fat body content of Stim-Ttl On flies increased by about $30 \%$ as compared to Stim-Ttl Off flies at day 1 after Ttl, and it further surged by $343 \%$ at day 11 , a value that was also 2.57 -fold higher than the value of 11 days post Stim- Ttl Off flies (Figure 11C). In contrast, the body fat content of Stim- TtI On flies in pair-fed condition only increased by $127 \%$ at day 1 , and it was also 1.85 -fold higher than observed with Stim- Ttl Off pair-fed flies at day 11 (Figure 11C). These results strongly suggest that not only increased food intake but also possible energy expenditure alterations may drive the development of t-LISO. 

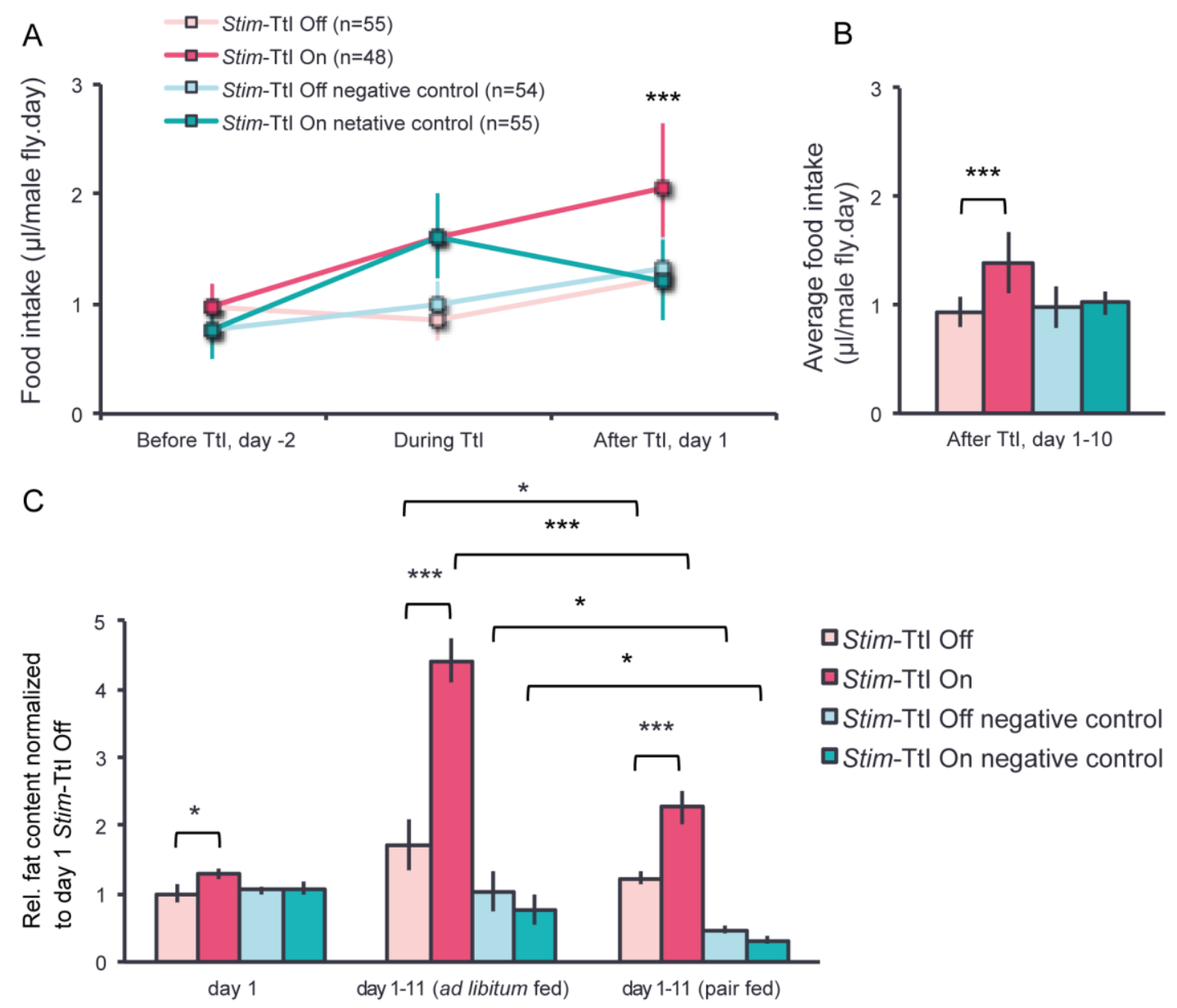

D

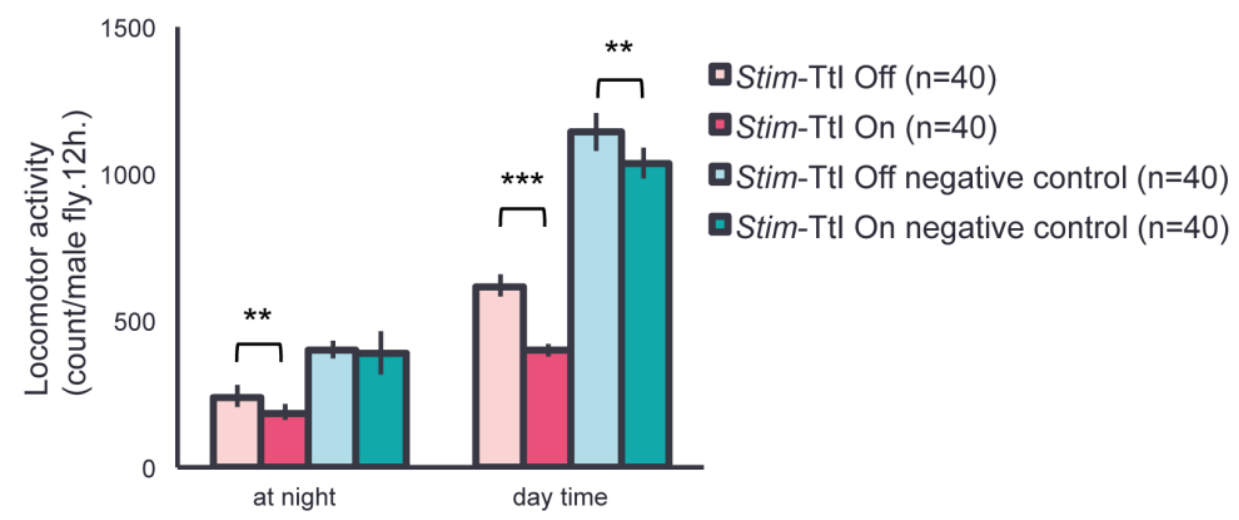

Figure 11. Increased food intake and less energy expenditure after Stim-TtI contribute to t-LISO

A. Food intake as revealed by the capillary feeding assay establishes that Stim-TtI On results in an increased food of male flies during Stim RNAi expression and at day 1 thereafter as compared to corresponding control (Stim-Ttl Off) flies. Stim-Ttl On negative control adult male flies also showed an increased food intake during the induction period, which, however, was declined to the same level as observed with the Stim-TI Off negative control flies at day 1. B. The averaged daily food intake indicates an increased average intake per fly over a period of 10 days after Stim-TtI On as compared to the corresponding control (Stim-Ttl Off) flies.

C. Quantification of the body fat contents of adult male Stim-TtI On flies (red)), and control males (Stim-Ttl On negative control flies (deep green), Stim-Ttl Off flies (light red), Stim-TtI 
Off negative control flies (light green)) at day 1 and day 11 with differentially feeding methods. Males were fed by capillary feeding, either provided an ad libitum food source (liquid food as much as flies liked) or offered restricted amounts of liquid food for different kinds of flies (pairfed condition). Relative (rel.) fat content is represented as fold change: TAG equivalent content/protein content of Stim-Ttl On/Off or Stim-TtI On/Off negative control adult male flies normalized with respect to the TAG equivalent content/protein content of day 1 Stim-Ttl Off adult male flies (standard value). Note that flies at day 11 had increased body fat storage under both ad libitum feeding and pair-fed conditions, and that this increase was higher in ad libitum fed flies than in pair-fed flies. For details of CAFÉ assay see 2.5.5.

D. Locomotor activity assay shows that after Stim-TtI On, the Stim-TI On flies showed both reduced day-time activity and reduced night-time locomotor activities as compared to the corresponding control (Stim-Ttl Off) flies. For details of locomotor assay see 2.5.6.

For details of the nomenclature see legend of Figure 3. The Stim RNAi1 transgene line was used to express Stim RNAi. Error bars represent the standard deviation of samples. ${ }^{*}=$ $p<0.05,{ }^{* *} ;=p<0.01,{ }^{* *}=p<0.001$ (Student's t-test).

Physical activities is the most variable component of the daily energy expenditure (Hall et al., 2012; Ravussin and Swinburn, 1992). Lack of physical activity due to sedentary life styles is strongly linked to the prevalence of obesity (Church et al., 2011; Hu et al., 2003; Livingstone et al., 2003; Roda et al., 2016). Reduced locomotor activity is a major contributor of high fat diet induced obesity in mice (Bjursell and Gerdin, 2008). In Drosophila, locomotor activity has been successfully quantified by an actometer test (Liu et al., 2008). The link between locomotor activity and obesity has not been identified (Baumbach et al., 2014a; Bharucha et al., 2008; Liu et al., 2012). To exclude the possible impact of temperature and/or the circadian rhythm on Stim-dependent obesity, I monitored and quantified the locomotor activities of flies during the day and during night-time. Figure 11D shows that after Stim-Ttl On, the flies showed both reduced day activity and reduced night locomotor activities as compared to the corresponding control (Stim-Ttl Off) flies. The locomotor activities of t-LISO (Stim-TtI On) at night time was by about $30 \%$ lower than observed with Stim-Ttl Off control flies, and about $56 \%$ lower during day time. This value is much larger than the difference observed with a temperature shift alone (Figure 11D). Additionally, the reduction of locomotor activity occurred as early as 3 days after Stim-Ttl (Supplementary Figure 23C, D). These results imply the possibility that reduced locomotor activity, which results in less energy expenditure, also contributes to the development of t-LISO.

Taken together, the results show that the increase of food intake and decrease of locomotor activity are likely to be the major contributor to t-LISO development. 


\subsubsection{Dysfunctional energy homeostasis after Stim-TdI contributes to d-LISO}

To confirm the energy imbalance phenotype during d-LISO development, I monitored the energy intake and metabolic rate of Stim-Tdl On and Stim-Tdl Off flies. Similar to the results shown in Figure 11, the food intake of d-LISO flies also had 34\% more than Stim-Tdl Off flies as early as day 2 after Tdl, and maintained at least $45 \%$ higher food intake during the following seven days (Figure 12A). On average, the daily food intake amount of $d$-LISO flies was about 1.5 -fold higher than the value of Stim-Tdl Off flies from day 1 to day 7 (Figure 12B).

Apart from the calorie intake, the diet composition had also an impact on the fat storage of the fly (Skorupa et al., 2008). To determine whether the diet composition contributes to d-LISO, Stim-TdI On/Off flies were assayed on food with different concentrations of yeast and sucrose, respectively. Figure $12 \mathrm{C}$, shows that malnutrition diet containing low yeast and low sugar concentrations did not prevent the fat content increase of $d$-LISO flies by about 7 fold as compared to the controls, moreover the yeast/sugar ratio did not significantly affect the body fat content of the d-LISO flies. The data indicate that the low nutrient value or different sugar/yeast ratios do not prevent the d-LISO development.

Given the increased food intake of d-LISO flies, I asked whether the extra non-lipid diet could be quickly transformed into stored lipid classes (neutral lipids and polar lipids) in those flies. To answer this question, flies was subjected to $\mathrm{C}^{14}$ labeled glucose pulse feeding, which is directly followed by lipid incorporation analysis (Katewa et al., 2012). I examined the $\mathrm{C}^{14}$ incorporation rate in neutral and polar lipid fractions after feeding Stim-Tdl On/Off adult male flies on day 4 after Stim-Tdl On activity for 24 hours with food containing ${ }^{14} \mathrm{C}$ labeled glucose (Figure 12D). Although different nutrients can be converted into fat (Al-Anzi et al., 2009), this method can provide a overview of how glucose intake can be converted into lipid biosynthesis processes, and it allows to assess the relative $\mathrm{C}^{14}$ lipid incorporation rates into de novo synthesized lipids in Stim-Tdl On and Stim-Tdl Off males. Indeed, neutral lipid incorporation rate of $d$-LISO was almost five times higher than the value of Stim-Tdl Off flies (Figure 12E). Furthermore, the polar lipid incorporation rate in d-LISO flies was $79 \%$ higher than in the corresponding control flies (Figure 12F). Hence, a likely higher food intake level is sufficient to increase lipid biosynthesis of d-LISO flies. 

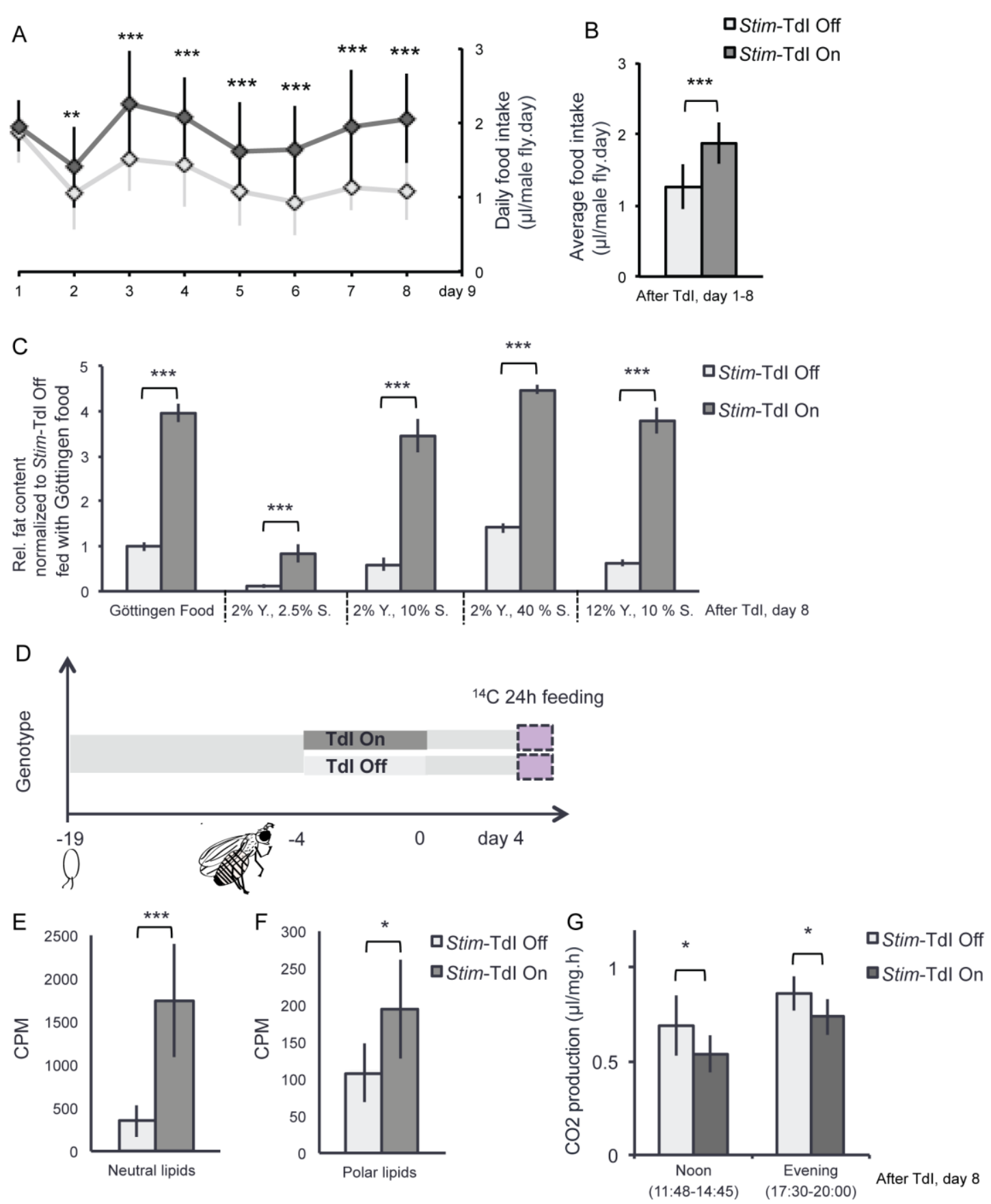

Figure 12. Increased food intake, increased lipid biosynthesis and less energy expenditure after Stim-Tdl contribute to d-LISO

A, B. Food intake using the capillary feeding assay revealed that Stim-Tdl On results in an increased daily food intake of male flies from day 2 to day 8 when compared to the corresponding control (Stim-Tdl Off) males (A). Note that the averaged daily food intake was also increased over a period of 8 days after the induction time point (B).

C. Quantification of the body fat content at day 8 after Stim-Tdl On in adult male flies as compared to corresponding control (Stim-Tdl Off) flies on different diets. Except normal Göttingen food, the food was $1 \%$ agarose in water as well as the indicated ingredients $(Y .=$ Yeast; S.= Sucrose) were added. \% refers to $\mathrm{w} / \mathrm{w}$. Relative (rel.) fat content is represented as fold change: TAG equivalent content/protein content of Stim-Tdl On flies normalized with respect to the TAG equivalent content/protein content of Stim-Tdl Off flies fed with Göttingen 
food. Note that the body fat content of the Stim RNAi expressing flies is increased and that this effect is independent of the diet compositions. For details of CAFÉ assay see 2.5.5.

D. Experimental scheme showing the time point of ${ }^{14} \mathrm{C}$ pulse chase labeling of the food intake of Stim-Tdl On and Stim-Tdl Off adult male flies. For this experiment, ${ }^{14} \mathrm{C}$-labeled glucose was added to the food as described in Material and Methods (2.3.6) and the flies were fed for 24 hours with Göttingen food supplemented with the ${ }^{14} \mathrm{C}$-labeled glucose on day 4 after the Stim-Tdl On and directly experimented for ${ }^{14} \mathrm{C}$ lipid incorporation assay.

E. ${ }^{14} \mathrm{C}$-label analysis in the neutral lipid and polar lipids extracts of Stim-Tdl On and Stim-Tdl Off adult male flies indicates that the Stim-Tdl On flies had a significantly higher ${ }^{14} \mathrm{C}$ incorporation into $(\mathbf{E})$ neutral lipids and $(\mathbf{F})$ polar lipids than the corresponding control flies by the end of the labeling period. $\mathrm{cpm}=$ counts per minute of lipid extractions of 20 flies.

G. $\mathrm{CO}_{2}$ measurements indicate that 8 days after Stim-Tdl On in adult male flies at noon and evening (around $2.5 \mathrm{~h}$ at each time point), the metabolic activity was reduced when compared corresponding control (Stim-Tdl Off) flies. For details of $\mathrm{CO}_{2}$ assay see 2.5.7.

For details on the nomenclature of Stim-Tdl On, Stim-Tdl Off, please see Figure 4. Stim RNAi1 transgene line was used in following studies. Error bars represent the standard deviation of samples. ${ }^{*}=p<0.05,{ }^{* *} ;=p<0.01,{ }^{* * *}=p<0.001$ (Student's t-test).

Since energy expenditure includes physical activity, basal metabolism, and adaptive thermogenesis (Spiegelman and Flier, 2001), I also set out to determine whether the metabolic rate was changed in d-LISO flies. I used the $\mathrm{CO}_{2}$ production assay which has been used to indicate differences in the metabolic rate in fly (Takeuchi et al., 2009; Yatsenko et al., 2014). Consistent with the reduction in locomotor activity of tLISO flies, at noon and evening time, I observed that there was a $29 \%$ and a $17 \%$ decrease in the $\mathrm{CO}_{2}$ production of d-LISO flies, respectively, when compared to the corresponding control flies. Thus, it appears that the energy expenditure is reduced in d-LISO flies.

In summary, the results indicate that d-LISO flies exhibited persistent overfeeding, increased lipid biosynthesis and a reduced metabolic rate. These findings support the argument that a mismatch of the energy balance leads to the d-LISO phenotype.

\subsection{Molecular, cellular and system investigations on mechanisms underlying the LISO}

I have sequentially established a causal link between Stim-TtI, Stim-Tdl and a chronic energy imbalance that goes along with Stim impairment and LISO. In order to address the mechanisms underlying LISO development, I addressed the following two questions. (1) How does Stim-Ttl induce long-term reduction of Stim expression and thus a long-term impairment of calcium signalling? (2) How does the impairment 
of calcium signalling result in energy imbalance of flies and their abnormal energy storage?

\subsubsection{Mechanism of long-term reduction of gene mRNA expression after Ttl}

To answer the question of how was long-term reduced Stim expression caused by Stim-Ttl, or Stim-Tdl, collectively referred to as Stim-Transient Induced RNAi expression (TI), I addressed two hypotheses. First, Stim-TI induces a long-term Stim knockdown of the mRNA. Second, Stim-TI programs Stim transcriptional repression. If the first proposal was correct, one could expect that the critical player of RNAi process, the small interfering dsRNAs (siRNAs) continuously target the Stim mRNA and thus, RNAi must remain at high or at least measurable levels after TI. If this was not the case, the second hypothesis would be more likely.

Hence, I needed to monitor how shorter interference dsRNAs (siRNAs) degrade after transient RNAi induction. It would be ideal that I can directly check siRNA's level in post Stim-Ttl flies. However, the Stim RNAi line I used expresses 343bp long double stranded RNA (dsRNA) which makes it difficult to identify the one or several siRNA, of the possibly many different siRNAs which are generated from a dsRNA transgene (Eid et al., 2008).

A
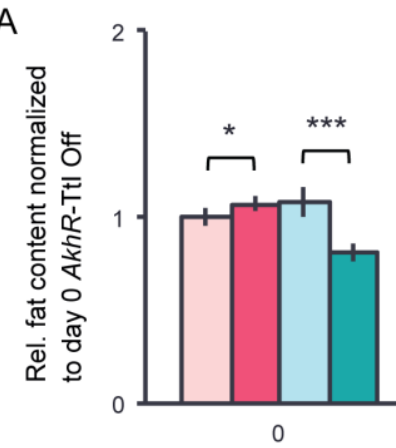

B
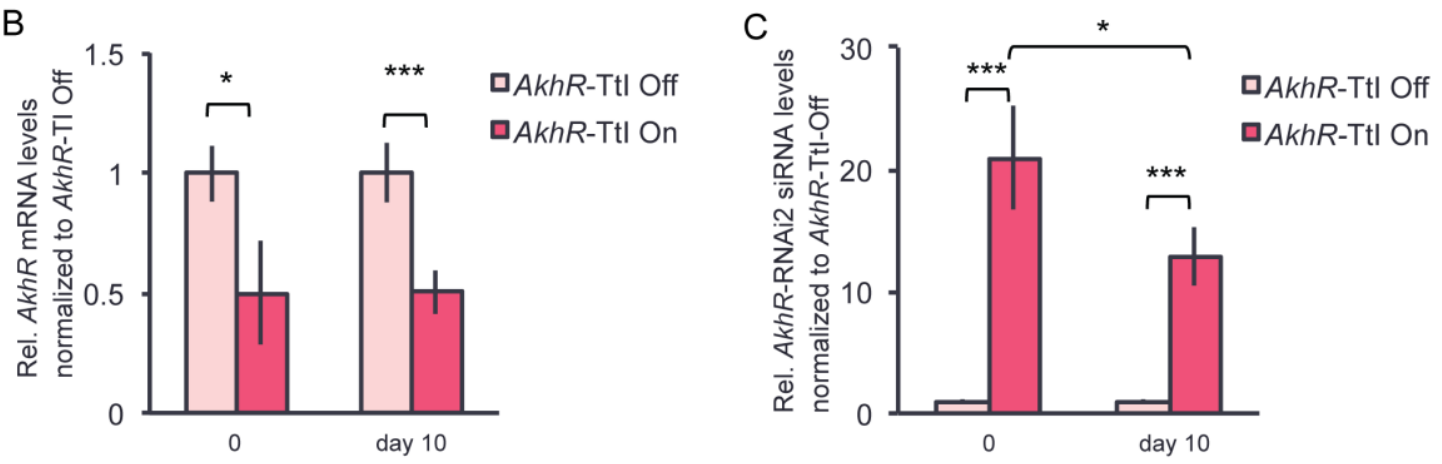

Figure 13. Long-term existence of siRNAs from $A k h R-T t l$ and their effects on body fat storage and AkhR mRNA expression 
A. Quantification of the body fat contents on day 1 and days 21 after AkhR-Transient temperature-shift Induced RNAi expression for $48 \mathrm{~h}(A k h R$-Ttl On) in adult male and female flies, and on corresponding control ( $A k h R$-Ttl Off, $A k h R-T t l$ On negative control, AkhR-Ttl Off negative control) flies. Relative (rel.) fat content is calculated as fold changes: TAG equivalent content/protein content of $A k h R-T t l$ On/Off or $A k h R$-Ttl On negative control flies normalized with respect to the TAG equivalent content/protein content of day 1 AkhR-TI Off (standard value). Note an only minor but significant long-term increase of body fat at day 21 after the induction time point of $A k h R$-Ttl On ( $A k h R$-short hairpin RNAi, AkhR-RNAi2). For details of genetic constitution of flies and experiment procedures see 2.1.1, 2.1.2,2.1,4, 2.3.1, 2.3.3.

B. RT-qPCR analysis shows that the fly abdomen $A k h R$ mRNA expression level reduced at day 1 day and day 10 after $A k h R$-TI On as compared to AkhR-TI Off males. Relative (Rel.) mRNA levels are represented as fold change, which refers to the basis value $(=1)$ obtained from AkhR-Ttl Off flies; Act5C mRNA served an internal standard and was used as reference to normalize the levels of above mentioned gene mRNA expression. For details of RT-qPCR see 2.2.4.1.

C. TaqMan RT-qPCR analysis shows that $A k h R$ RNAi effector (siRNA) was efficiently produced from the $A k h R$-shRNAi transgene at day 0 after the induction, and was maintained at significant high levels in the abdomen of $A k h R$-TI On males when compared to AkhR-TI Off males on day 10. Relative (Rel.) mRNA levels are represented as fold change, which refers to the basis value ( $=1$ ) obtained from AkhR-Ttl Off flies; $A k h R$ RNAi effector (siRNA) levels were normalized to the value of small RNA internal control $2 s$ rRNA. For details of TaqMan RTqPCR see 2.2.4.2 and table 4. AkhR RNAi2 transgene line was used in above studies. Error bars represent the standard deviation of samples. ${ }^{*}=p<0.05,{ }^{* *} ;=p<0.01,{ }^{* *}=p<0.001$ (Student's t-test).

Theoretically, siRNAs (21-23 nucleotides) are randomly generated (Zamore et al., 2000), it is difficult to pin down which one of siRNAs really knockdown Stim's mRNA level. In addition, Prof. Dr. R. Kühnlein and I realized that short hairpin RNA (shRNA) mediated RNAi lines generated by Harvard TRiP project have short target sequence as equal as nucleotide number of siRNA (Perkins et al., 2015), which can be detected by Taqman RT-qPCR (Jung et al., 2013). I decided to find a shRNA RNAi line for siRNA detection, which is effective enough in increasing the fly's body fat accumulation. Therefore, I used instead of the $A k h R$ shRNA RNAi transgenic line that expresses temperature-inducible $A k h R$ RNAi $(A k h R-T \mathrm{tl})$. Figure 13A shows that the AkhR-Ttl On shRNA RNAi line (Perkins et al., 2015) rendered a persistent and significantly higher body fat content after transient RNAi induction as compared to control flies. AkhR-Ttl caused an increase of $7 \%$ over the fat content of $A k h R$-Ttl Off control flies at day 0 , and it rose to a 1.23-fold increase up until day 21 (Figure 13A). It is to note that the temperature shift alone ( $A k h R-T t I$ On negative control) caused a $20 \%$ reduction of body fat content in following 10 days after Ttl. This observation suggests that the increase of body fat in AkhR-Ttl On flies might be actually higher if one takes the fat reduction in response to the previous temperature shift into account 
(Figure 13A). Accordingly, AkhR mRNA levels in AkhR-Ttl On flies were reduced by about $50 \%$ at day 10 after the induction of the AkhR RNAi (Figure 13B). TaqMan RT-qPCR revealed that in AkhR-Ttl On flies, AkhR siRNA level was 20.96 fold higher at day 0 when compared to $A k h R$-Ttl Off flies; and the siRNA level remained 12.86 fold higher at day 10 , but it was reduced when compared to the value at day 0 (Figure 13C). These data indicate that siRNAs generated by AkhR Transient RNAi Induction (TtI) are degraded over time, but the long lasting AkhR RNAi is obviously sufficient to drive a long-term impact on the AkhR mRNA target.

Taken together, these findings reveal that the siRNAs generated from AkhR-TtI maintains a sufficiently high level in fat storage tissue to cause long-term knockdown of $A k h R$, which indicates the similar mechanism existing in Stim-Ttl flies.

\subsubsection{RNA-seq analysis reveals candidate regulator genes in fat body tissue during LISO development.}

Food intake was found essential for the development of LISO flies. Furthermore, physiological data indicated that less energy expenditure also contributes to LISO development. To get insight into the molecular mechanisms underlying LISO development, RNA-seq analysis was used for transcriptome profiling (Daines et al., 2011; Graveley et al., 2011; Marioni et al., 2008; Wang et al., 2009). To have a unbiased overview on gene expression changes during t-LISO development, I performed RNA-seq profiling on dissected fat body tissues from day 10 Stim-TtI On and Stim-Ttl Off adult male flies, when LISO was still developing (Figure 14A). Both clustering analysis and principal component analysis (PCA) with $R$ based on read counts data show a separation of Stim-Ttl On fly samples and Stim-Ttl Off fly samples, which documents a good quality of RNA-seq experiments (Figure 24 (Supplementary data) A, B). On the basis of gene differential expression analysis with DESeq2 (Love et al., 2014), there were 2,548 differentially expressed genes $\left(\log _{2}\right.$ Fold change $\geq 0.5$, adjusted $p$ value $<0.05$ ) of which 1,401 genes were upregulated and 1,147 were down-regulated (DVD, Appendix 5.). Consistent with RTqPCR results, RNA-seq analysis also showed the down-regulation of Stim mRNA expression. I further conducted Gene Ontology (GO) analysis by FlyMine on upregulated and down-regulated genes. Using a $p$ value $<0.05$ for gene selection, three kinds of GO terms were examined: biological processes, the cellular components they represent, and their molecular functions. 
In terms of up-regulated genes, the majority of genes appear to be involved in cilium assembly or movement, microtubule based movement, and anion transport biological process (Figure 24 (Supplementary data) C). Accordingly, related cellular component GO terms revealed that the genes encode cilia components, components of the extracellular space, the cytoskeleton, the dynein complex, and membrane component which are significantly enriched (Figure 14B).

day 10 Stim-Ttl On/Of
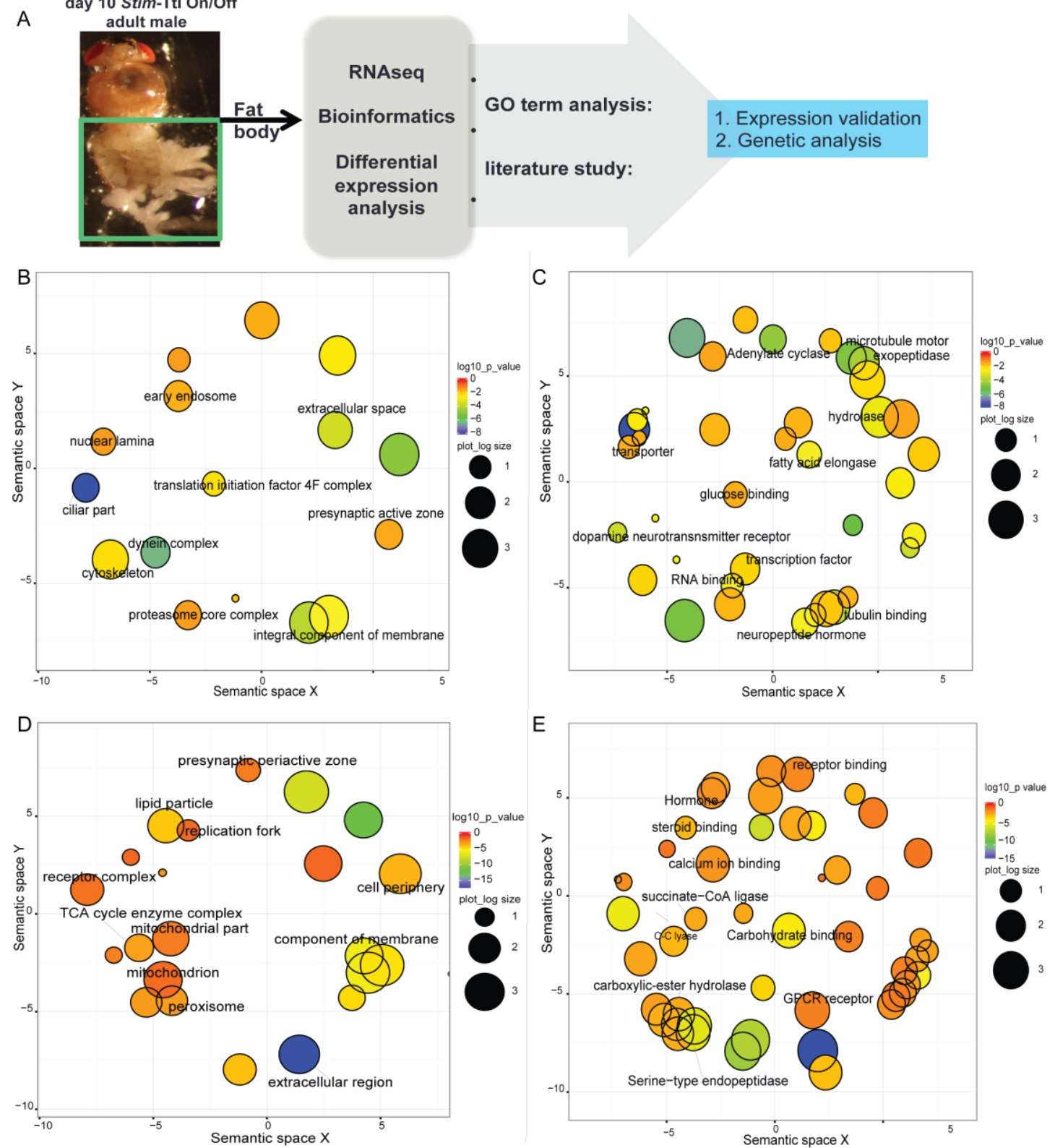

Figure 14. RNA-seq analysis identifies gene expression changes in LISO fly fat body tissue

A. Scheme showing the procedure of RNA-seq analysis of abdominal fat body tissues at day 10 Stim-Ttl On and Stim-Ttl Off (control) male flies after Stim-TtI. RNA of the dissected abdominal fat body tissues was extracted, and RNA-seq was performed and RNA-seq data subjected to bioinformatic analysis as described in 2.2.3. Differentially regulated candidate 
genes were selected on the basis of their $\log _{2}$-fold change $>0.5$ or $<-0.5$, and $p$ value $<0.05$ (wald test), respectively, and further analysed by the Gene Ontology (GO) term enrichment analysis ( $p$ value $<0.05$ ). Based on literature study, candidate genes expression was further validated by RT-qPCR, gene functions were further analysed with genetic analysis. Stim RNAi1 transgene line was used in the RNA-seq studies.

B.C. Cellular component and molecular function GO term analysis were carried out on the upregulated gene. B. Cellular component GO terms refer to genes, which encode proteins located at the early endosome, the nuclear lamina, the extracellular space, the cilia, the translation initiation factor $4 \mathrm{~F}$ complex, the presynaptic active zone, the cytoskeleton, the dynein complex and the proteasome complex. C. Molecular function groups refer to genes which encode adenylate cyclases, microtubule motors, exopeptidases, hydrolases, transporters, fatty acid elongases, glucose binding proteins, dopamine receptors, transcription factors, RNA binding proteins, neuropeptide hormones, tubulin binding proteins.

D.E. Cellular component and molecular function GO term analysis were carried out on the down-regulated gene. D. Cellular component GO terms refer to genes, which encode proteins located at the presynaptic periactive zone, at lipid particles, at the replication fork, at the receptor complex, at the TCA cycle enzyme complex, at mitochondria, at component of membranes, at the peroxisome, as well as at the extracellular space. E. Molecular function GO term analysis refer to genes, which encode receptor binding proteins, hormones, GPCR receptors, steroid binding proteins, calcium binding proteins, succinate-CoA ligases, C-C lyase, carxylic-ester hydrolases and serine type endopeptidases. Note that each circle represents one GO term (represeantative GO terms were labeled); color code indicated $p$ value, the scale is defined by $\log _{10}(p$ values); circle size indicates the gene numbers in each GO term, the scale is defined by $\log _{10}$ (gene numbers in each GO term).

In addition, genes encoding tubulin-binding proteins, microtubule motor or transporters are the most significant molecular function GO terms (Figure 14C). Besides, the translation initiation factor $4 \mathrm{~F}$ is also one of the significantly enriched molecular function GO term. These results indicate the possibility that tublin binding proteins and microtubule motor related genes are involved in LISO development. Interestingly, components involved in cAMP synthesis and degradation biological processes, as well as for (G protein-coupled receptor) GPCR signalling were also highlighted (Figure 24 (Supplementary data) C). Accordingly, molecular function GO terms such as the adenylate cyclase, the GPCRs, and the neuro-peptide hormone are also enriched (Figure 14C). More specifically, expressions of GPCR gene $A k h R$, and neuropeptide genes $\mathrm{CCHa} 2$ (encoding peptide hormone CCHamide2) and Tk (encoding neuropeptide Tachykinin) are significantly upregulated, which may reflect the likely down-stream regulatory network change of these two genes.

Most of the down-regulated genes correspond to metabolic processes in biological process GO term analysis such as catabolic lipid and carbohydrate metabolic 
processes as well as ATP generation (Figure 24 (Supplementary data) D). Consistently, cellular component GO terms such as lipid particles, mitochondria, and peroxisome were significantly enriched terms (Figure 14D). Moreover, most downregulated genes belong to molecular function GO terms, which define activities such as carboxylic-ester hydrolase, oxidoreductase, succinate-CoA ligase (tricarboxylic acid cycle (TCA) enzyme) and peptidase activity (Figure 14E). Hence, it is possible that down-regulation of energy metabolism might occur during LISO development. Finally, it is noteworthy that significant enriched molecular function GO terms in down-regulated genes also include genes which encode hormones, steroid and calcium binding proteins, such as the insulin antagonist gene ImpL2. These findings provide clues of how long-term impairment of calcium signalling from Stim-TtI may interfere and/or regulate metabolic processes that contribute to LISO. Overall, these results provide us with a first understanding of the transcriptional changes during the development of LISO flies showing that hormone genes, lipid metabolism genes, mitochondria genes are affected in response to the Stim Transient Induced RNAi expression.

\subsubsection{Akh signalling contributes to LISO via increased food intake}

Baumbach et al. reported that acute Stim knockdown in the fat storage tissue caused hyperphagia partially via sNPF up-regulation in the brain (Baumbach et al., 2014a). Thus, I sought to identify hormone-signalling components in addition to sNPF, which are expressed in brain or other peripheral organs and are likely involved in the regulation of overeating of LISO flies. To identify genes that encode such components, I did a small screen by RT-qPCR, asking whether the expression levels of food intake related neuropeptide or neurotransmitter candidate genes changed in in the heads of Stim-Ttl On flies at day 10 after Stim-Ttl. It included the Tryptophan hydroxylase gene Trh, which encodes the enzyme functioning as the first and ratelimited step in the synthesis of serotonin (5-HT) (Coleman and Neckameyer, 2005), the 5-hydroxytryptamine (serotonin) receptor 7 gene 5-HT7, encoding a GPCR serotonin receptor and transmit serotonin signaling (Becnel et al., 2011); the mammalian orexipeptide NPY homolog gene NPF (Brown et al., 1999), the mammalian corticotrophin-releasing hormone $(\mathrm{CRH})$ homolog- Diuretic hormone 44 gene Dh44 (Cabrero et al., 2002; Dus et al., 2015), the neuropeptide corazonin gene Crz (Hergarden et al., 2012; Veenstra, 1994), the mammalian cholecystokinin (CCK) homolog-Drosulfakinin (DSK) gene Dsk (Nichols et al., 1988; Söderberg et al., 2012), the vertebrate calcitonin gene-related peptide (CGRP) homolog-Diuretic hormone 31 
gene Dh31 (Coast et al., 2001; Hewes and Taghert, 2001; Kunst et al., 2014), the CCHamide peptide gene CCHa2 (Ida et al., 2012; Ren et al., 2015), the SIFamide neuropeptide gene SIFa (Park et al., 2014b; Verleyen et al., 2004). Out of these nine candidate genes tested, the expressions levels of NPF, Dh44, and Crz mRNA were significantly increased in day 10 Stim-Ttl On fly head tissue (Figure 25 (Supplementary data) A). At day 1 however, only $\mathrm{Crz}$ mRNA was significantly upregulated (Figure 25 (Supplementary data) A.B). The control experiments showed, however, that the up-regulation of $\mathrm{Crz}$ mRNA was also observed in the corresponding temperature-shift treatment control (Stim-TtI On negative control) flies (Figure 25 (Supplementary data) C). Thus, the up regulation of $\mathrm{Crz}$ is likely due to temperature-shift rather than due to Stim RNAi expression. This shows that none of the food intake related neuropeptide or neurotransmitter candidate genes mRNA expressions are likely to be under the control of Stim activity.

Next, I asked whether peripheral food intake regulator genes might be affected in response to Stim RNAi expression. The Akh gene of Drosophila encodes a functional homolog of the human glucagon or $\beta$-adrenaline signalling genes. It has recently been shown that the food intake of $A k h$ loss-of-function mutants is decreased, while Akh ectopic overexpression augmented the food intake (Gáliková et al., 2017). In addition, it has been shown that the fly brain has Akh-responding neurons, called interoceptive SEZ neurons (ISNs). A decrease of $A k h R$ in ISNs reduced the sugar consumption (Jourjine et al., 2016). Given the increased food intake in LISO flies, I asked whether there is a positive correlation between Stim-Ttl and altered Akh signalling during LISO development. To determine whether the mRNA of the key components of the Akh signalling pathway was up-regulated during LISO development, I first performed RT-qPCR at day 1 and day 10 of Stim-TtI On/Off male flies. No Stim-dependent difference was observed with respect to the expression levels Akh mRNA among different flies (Figure 15A). Next, I asked whether the expression of the Akh receptor (AkhR) mRNA is altered in the Stim-Ttl On/Off male flies at day 10. Figure 15B shows that at day 10 the amount of AkhR mRNA was increased by about $50 \%$ in fat body tissues in Stim-TtI On as compared to the control flies. No such effect was observed with the expression of AkhR downstream gene target of brain insulin (tobi) (Buch et al., 2008) (Figure 15B). My RNA-seq data point to the increased expression of Tachykinin peptide gene (TK) by $113 \%$ (Figure 15C). Tachykinin has been linked to the release of Akh in locust (Nässel et al., 1995, 1999). In order to analyze whether a possible Tachykinin regulated Akh secretion occurs, I further reasoned that Akh signalling might be regulated on post-transcription 
level, which would promote feeding through brain but fail to mobilize lipids because of fat body tissue Akh resistance.

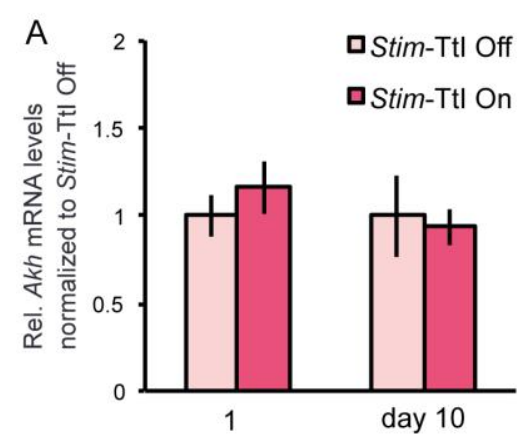

C

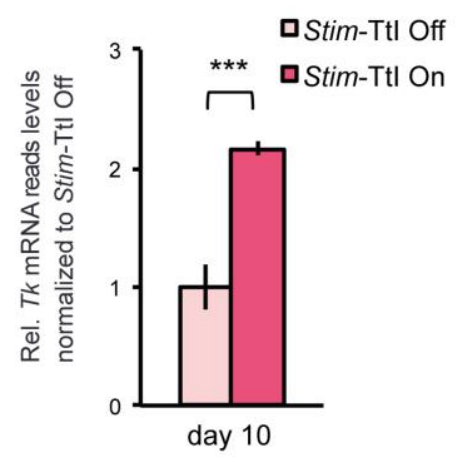

$\mathrm{E}$

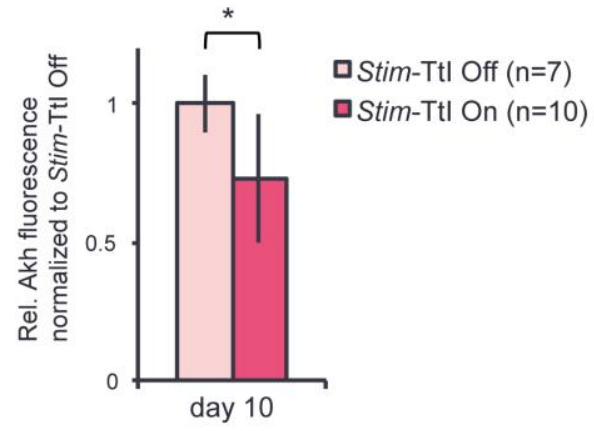

G

$-\diamond-$ Stim-Tdl Off in Akh homozygote mutant

- - Stim-Tdl On in Akh homozygote mutant

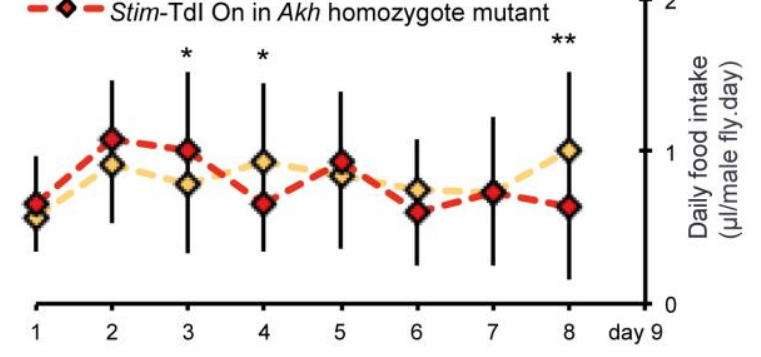

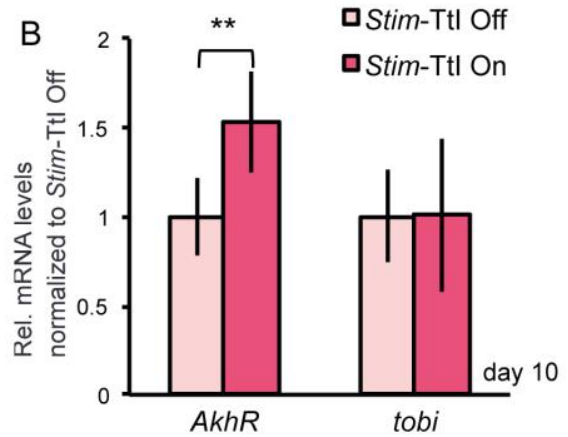

D day 10 Stim-Ttl Off

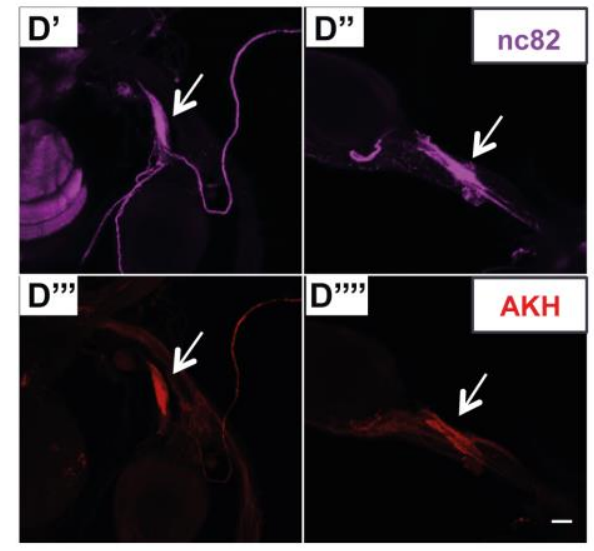

F $\quad \square$ Stim-Tdl Off in Akh heterozygote mutant

$\square$ Stim-Tdl On in Akh heterrozytoge mutant

$-\diamond-$ Stim-Tdl Off in Akh homozygote mutant

- - Stim-Tdl On in Akh homozygote mutant

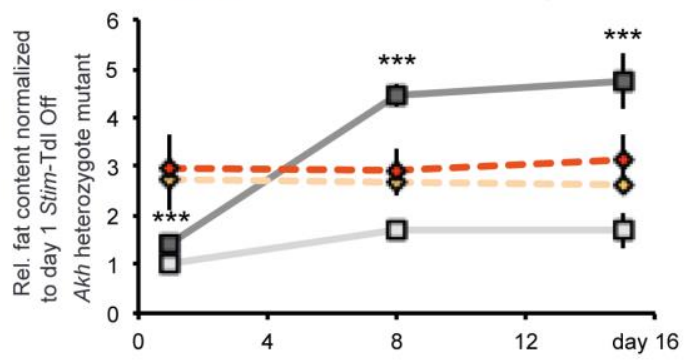

$\mathrm{H}$

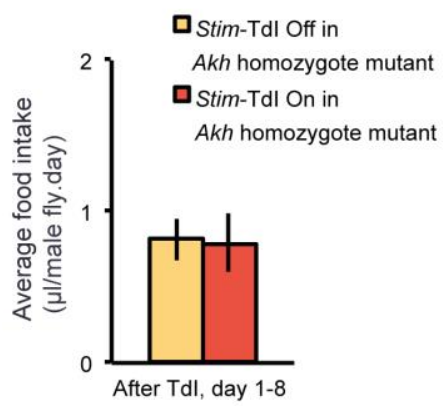

Figure 15. Akh signalling contributes to LISO in response to Stim-Ttl by promoting the food intake

A.B. Quantitative RT-qPCR analysis of $A k h$ in whole male fly and $A k h R$ in adult male fat body tissue after Stim-Ttl. A. The Akh mRNA expression level in adult male flies was not 
significantly affected at day 1 and 10 after the $38 \mathrm{~h}$ of Stim-Ttl On as compared to corresponding control adult male (Stim-Ttl Off) flies. B. The AkhR mRNA expression level was significantly increased but not the mRNA expression level of $A k h R$ downstream target gene tobi in adult male fat body tissues at day 10 after $38 \mathrm{~h}$ of Stim-Ttl On as compared to corresponding control adult male (Stim-Ttl Off) flies. Relative (Rel.) mRNA levels are represented as fold change, which refers to the basis value $(=1)$ obtained from Stim-Ttl Off flies; Act5C mRNA served an internal standard and was used as reference to normalize the levels of above mentioned gene mRNA expression.

C. RNA-seq raw reads counting indicates that the mRNA expression level of neuropeptide gene Tachykinin (Tk) was significantly increased in adult male fat body tissues at day 10 after $38 \mathrm{~h}$ of Stim-Ttl On.

D. Immunostaining of the Akh peptide (red) by Anti-Akh antibodies to visualize the corpora cardiaca of adult male flies at day 10 under control condition (Stim-Ttl Off; D'”) or at day 10 after $38 \mathrm{~h}$ of Stim-TtI (Stim-TI On; D',''). Anti-Bruchpilot antibodies nc82 were used to identify the corpora cardiaca neurons (D' and D'"). Scale bar in D'"' represents $25 \mu \mathrm{m}$. All micrographs (D-D"',') were taken with the same magnification

E. Quantification of the Anti-Akh antibody fluorescent staining in corpora cardiaca cell area shows that the Akh staining is reduced in the corpora cardiaca of day $10 \mathrm{Stim}$-Ttl On adult male flies as compared to corresponding control adult male (Stim-Ttl Off) flies. For details of Akh immunostaining see 2.4.5.

F. Quantification of the body fat content at day 1, 8, and 15 after Stim-Tdl On in adult male flies as compared to corresponding control (Stim-Tdl Off) flies. Flies were either heterozygous or homozygous Akhmutant males. Relative (rel.) fat content is represented as fold change: TAG equivalent content/protein content of Stim-Tdl On/Off flies normalized with respect to the TAG equivalent content/protein content of day $1 \mathrm{Stim}$-Tdl Off flies in Akh heterozygous (serving as reference). Note that the obesity phenotype develops after Stim-Transient drugfeeding induced RNAi expression (Stim-Tdl On) in heterozygous but not in homozygous Akh mutant males.

G. H. Food intake using the capillary feeding assay revealed that Stim-Tdl On in homozygous Akh mutant male flies did only weakly change the food intake from day 1 to day $8(F)$ when compared to the corresponding control (Stim-Tdl Off in homozygous Akh mutant) males after the induction time point $(\mathbf{A})$. Note that the averaged daily food intake over a period of 8 days after the induction was not changed (G). For details of genetic constitution of flies and experiments see 2.1.1, 2.1.2, 2.1.5, 2.1.7, 2.2.2, 2.3.1, 2.3.3. Stim RNAi1 transgene line was used in above studies. Error bars represent the standard deviation of samples. ${ }^{*}=p<0.05$, ${ }^{*}$ $;=p<0.01,{ }^{* * *}=p<0.001$ (Student's t-test).

A previous study showed that Akh secretion from corpora cardiaca (CC) cells is promoted by CAMP activated kinase AMPK signalling during high-to-low sugar transition (Braco et al., 2012). The inverse relationship between Akh immunostaining of CC cells and Akh secretion has been identified (Braco et al., 2012; Kim and Neufeld, 2015). Hence, I tested the possible hypothesis that Stim-Ttl in fat body tissue promotes food intake via non-autonomous up-regulation of Akh release. By using nc82 antibody and antibody targeting the Akh peptide (Kim and Rulifson, 2004), I did immuno-staining on isolated CC cells from day 10 Stim-TtI On/Off flies, 
using the nc82 antibodies directed against the Drosophila protein Bruchpilot to identify the CC cells, and anti-Akh antibodies to quantify the Akh storage level in CC cells (Figure 15D). The results show that Akh staining of Stim-Ttl On fly brains is reduced by $27 \%$ in comparison to Stim-Ttl Off flies (Figure 15E). The result suggests that Stim-Ttl in fat body tissue increases the Akh secretion from the brain via secreted Tachykinin.

To verify that Akh signalling is indispensable for LISO development, I performed genetic interaction experiments with Stim-Tdl and Akh loss of function mutant flies. Notably, just after Stim-Tdl, both Stim-Tdl On and Stim-Tdl Off flies, which were homozygous for the Akh loss of function mutation, developed a 2-fold higher fat storage than Akh heterozygous flies (Figure 15F), which is in line with the previous finding (Gáliková et al., 2015). Regarding Stim-Tdl On in Akh heterozygous mutant flies, their body fat content was increased more than 4-fold when compared with the corresponding control flies. However, Stim-Tdl On flies in Akh homozygous condition failed to accumulate more fat when compared to Stim-Tdl Off in Akh homozygous condition during the same time period (Figure 15F). These data support the conclusion that LISO development requires Akh signalling. I finally asked whether loss of $A k h$ function directly affects the food intake during LISO development. I therefore monitored the food intake of Stim-Tdl On and Stim-Tdl Off flies, which also carry Akh loss-of-function homozygous mutant. The results shown in Figures 15G, H indicate that the average food intake was not increased in response to Stim RNAi expression when Akh was totally absent. The results also suggest that the reduction of Stim activity in fat body cells provides a signal to be sensed by the CC cells, which regulates the secretion and/or the activity of Akh.

In summary, these results strongly support the notion that impairment of calcium signalling by Stim-Tdl caused more Akh secretions likely through Tachykinin signalling from fat body tissue, which further increase food intake and eventually contributes to the LISO development.

\subsubsection{Selective insulin signalling resistance occurs in LISO flies}

Combining differentially regulated genes identified by RNA-seq analysis with the previous physiological results, I reasoned that hormone signalling might be the key to of regulatory networks underlying the energy expenditure and storage changes of LISO flies. 
Insulin signalling regulator and target genes are significantly regulated. In Drosophila, as in humans, core components of insulin signalling pathway are conserved. They include insulin like peptide genes dllp-2, dllp-3, or dllp-5 (Brogiolo et al., 2001; Grönke et al., 2010). In addition, the CCHa2 receptor gene, CCHa2- $R$, the mammalian homologue of Bombesin receptor subtype-3 (Brs3) in Drosophila (Ida et al., 2012; Sano et al., 2015). Similarly, the insulin antagonist gene ImpL2, which resembles the Insulin-like growth factor-binding protein-7 (IGFBP7) in mammals (Honegger et al., 2008). The "decretin" hormone gene Limostatin (Lst) is the functional ortholog of mammalian Neuromedin $U$ in fly (Alfa et al., 2015). The insulin signaling pathway genes are also conserved such as insulin peptide receptor gene InR (Fernandez-Almonacid and Rosen, 1987), the PI3K catalytic subunit gene Dp110 (Leevers et al., 1996), the PI3K adaptor gene p60 (Weinkove et al., 1999), the Akt protein kinase gene Akt (Scanga et al., 2000), the TOR gene Tor (Oldham et al., 2000; Zhang et al., 2000), the S6 kinase gene dS6K (Montagne et al., 1999), and finally the transcription factor gene $d F O X O$ as well as the dFOXO target gene $d 4 E B P$ (Jünger et al., 2003; Kramer et al., 2003; Puig et al., 2003).

Insulin signalling regulates the number of fat body cells as well as fat accumulation in adult flies (DiAngelo and Birnbaum, 2009). Given the excessive fat accumulation of LISO flies, I asked whether changes in insulin signalling results in differential gene expression using the RNA-seq data obtained from Stim-TtI On and Stim-TtI Off flies. As shown in Figure 16A, genes encoding negative regulators of insulin signaling such as $I m p-L 2$ were down-regulated, while the gene encoding positive regulators of insulin signaling such as $\mathrm{CCH} 2$ and insulin signaling repressing target gene $d 4 E B P$ were significantly up-regulated (Figure 16A). I confirmed the differential expression of several genes related to insulin signaling by RT-qPCR with fat body tissue of StimTtl On and Stim-Ttl Off male males at day 10. The CCHa2 mRNA level showed an expression a significant increase at day 1 and was further increased by about $50 \%$ at day 10 (Figure 16B). In contrast, the expression level of ImpL-2 mRNA in Stim-TtI On was $84 \%$ lower than in Stim-Ttl Off flies at day 10 (Figure 16C). Similarly, the expression level of Lst (encoding a hormone peptide to suppress the insulin-like peptide secretion) mRNA was reduced by more than 3-fold as compared the corresponding control flies at day 10 (Figure 26 (Supplementary data) A).

It is known that a genetic disruption of $\mathrm{CCHa} 2$ severely reduced dILP-2 secretion from the Insulin Producing Cells (IPCs) of adult flies (Sano et al., 2015); while Imp-L2 
loss-of-function increased adult fly body size and might function as a potent dILP-2 inhibitor (Honegger et al., 2008). Taken these findings into account, a possible scenario is that Stim-Ttl might promote the dILP-2 secretion from IPC cells via upregulation of $\mathrm{CCHa}$, which further increases the fat mass of LISO flies. As expected, dILP-2 immunostaining of stored dILP-2 peptides in IPC cells suggests that the release of dILP-2 peptides in LISO flies is about $30 \%$ increased as compared to the corresponding control flies (Figure 16D, E).

However, I verify a significant increase of $d 4 E B P$ expression in the fat body tissue of day 10 LISO fly, despite the increased trend in d4EBP expression was also observed at day 1 (Figure 16F), a gene that which is normally repressed by active peripheral insulin signaling (Jünger et al., 2003; Puig et al., 2003). Next, I examined whether peripheral insulin signaling is up-regulated in the peripheral tissue of Stim-TdI On as compared to Stim-Tdl Off flies at day 4. I removed the heads of the flies and analysed the extracted proteins by Western blotting using two different antibodies directed against the active Akt protein, i.e. Akt, which is phosphorylated at position Ser505 and Thr342, respectively. Figure 16G and Figure 26 (Supplementary data) $\mathbf{F}$ show that the level of active Akt is not up-regulated. On the contrary, there is even a reduced Akt phosphorylation level (reduction is approximately 40\%) when compared with the corresponding control flies (Figure 16G, Figure 26 (Supplementary data) F). If there were increased insulin peptide secretion in the LISO brain, enhanced insulin signaling response such as increased cell proliferation should be observed in peripheral tissue. To test such an effect, I used EdU staining to visualize newly synthesized DNA in order to detect proliferation (FernándezHernández et al., 2013) in the fly fat body tissue. EdU staining did not reveal a significant difference in the fat body tissues of LISO and control flies (Figure 25 (Supplementary data) E), indicating that cell proliferation was not altered in response to Stim-Ttl.

Notably, however, the level of mRNA of the Hexokinase-C (Hex-C) gene, a homolog of the mammalian glycolytic enzyme Glucokinase (GCK), was substantially decreased in LISO fat body tissues (Figure 26 (Supplementary data) B). GCK is required for glucose clearance by the mammalian liver, which acts in the downstream of insulin signalling (Haeusler et al., 2015; Massa et al., 2011; Velho et al., 1996). Collectively, these results therefore suggest that insulin resistance has occurred in fat body tissues during LISO development. 

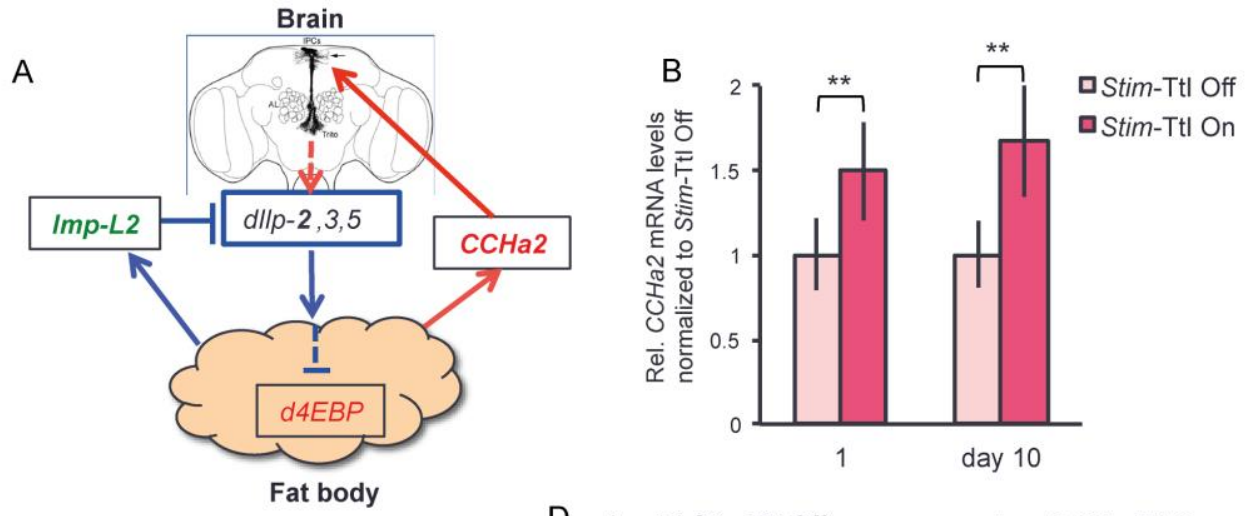

D day 10 Stim-Ttl Off
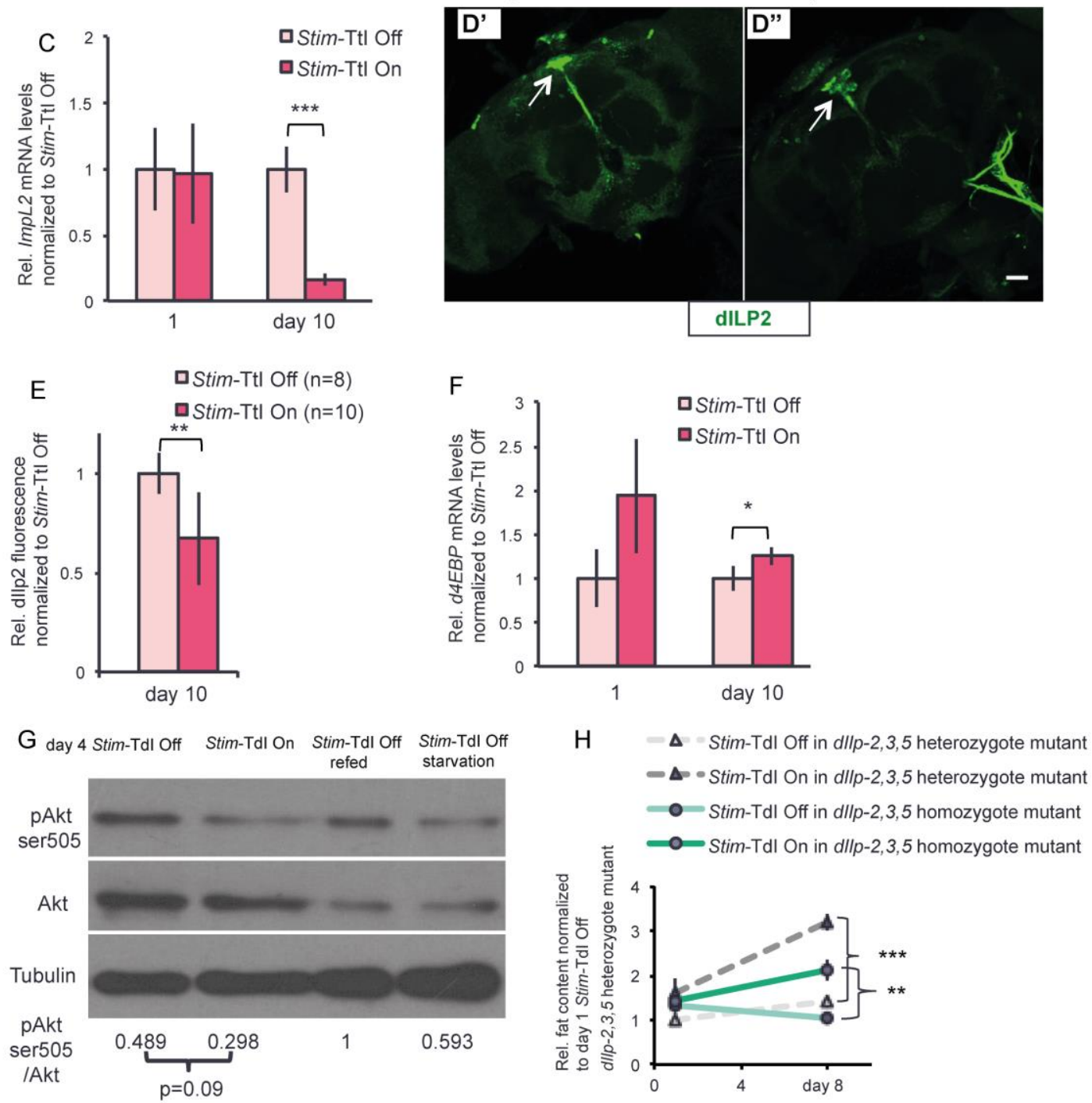

Figure 16. Insulin signalling resistance occurs in peripheral tissues of LISO adult males

A. Schematic representation of the regulation of insulin-like peptide secretion in the Drosophila brain as revealed by RNA-seq analysis. Candidate genes encode fat body secreted insulin-regulating factors in LISO condition such as the down-regulated Ecdysoneinducible gene L2 (ImpL2; green) and up-regulated CCHamide-2 gene (CCHa2) (red) at day 10 after Stim-TtI On. ImpL2 is a binding factor that inhibits insulin like peptide (dILP2) 
function, whereas the peptide hormone $\mathrm{CCHa} 2$ acts as a nutrient-dependent, and positive regulator of insulin-like peptide secretion in Drosophila.

B, C. Validation of the RNA-seq results (shown in Figure 14) by RT-qPCR confirms upregulation of mRNAs expression of the insulin secretion-promoting gene $\mathrm{CCHa2}(\mathbf{B})$ at day 1 and day 10, and down-regulation of mRNAs expression of the insulin peptide binding factor gene ImpL2 (C) at day 10 after Stim-TtI On in adult male fat body tissue as compared to the corresponding control (Stim-TtI On) flies. C. Note that the mRNAs expression of ImpL2 was not affected at day 1 after Stim-Ttl Off in adult male fat body tissue as compared to the corresponding control (Stim-Ttl Off) flies. Relative (Rel.) mRNA levels are represented as fold change, which refers to the basis value $(=1)$ obtained from Stim-Ttl Off flies; Act5C mRNA served an internal standard and was used as reference to normalize the levels of above mentioned gene mRNA expression.

D. Immunostaining of the dILP-2 peptide with anti-dlLP-2 antibodies (green) in insulin producing cells (IPC, indicated by white arrow) of adult fly brains at day 10 without (D') and after (D') the Stim-Ttl On. Scale bar in D" represents $25 \mu \mathrm{m}$. D. All micrographs were taken with the same magnification. E. Quantification of the dILP-2 staining fluorescence in the cell body area of IPCs in adult male brains shows that the dILP-2 is decreased at day 10 after the Stim-Ttl On when compared to the corresponding control (Stim-Ttl Off) male brains. For details of dlLP2 immunostaining refer to 2.4.5.

F. RT-qPCR analysis shows an increase of mRNAs expression of the insulin signaling gene $4 E B P$ at day 1 and day 10 after Stim-Ttl On in adult male fat body tissue. Quantification of gene relative (Rel.) mRNA levels refers to method described in B.C. For details of RT-qPCR see 2.2.4.1.

G. Western blot analysis showing the level of activated Akt (pAkt ser505; Ser 505 phosphorylation of Akt), Akt and the reference protein Tubulin in the day 4 Stim-Tdl On adult male peripheral tissues (fly tissues without head) and corresponding control (Stim-Tdl Off) fly peripheral tissues, and their accumulation in Stim-Tdl Off males with (after starvation, refed) and without (starvation) food supply. Note that pAkt ser505 appears to be reduced in response to the earlier Stim-Tdl On as observed with fed versus starved control flies. Quantification of the pAkt ser 505 as compared to the Akt levels (indicated below the slots) is based on integrated band intensities using the value of Stim-Tdl Off (refed) flies as reference. For details of Western blott see 2.3.1, 2.3.2.

H. Quantification of the body fat content at day 1, 8, and 15 after Stim-Tdl On in adult male flies as compared to corresponding control (Stim-Tdl Off) flies. Flies were either heterozygous or homozygous dllp2-3,5 mutant males. Relative (rel.) fat content is represented as fold change: TAG equivalent content/protein content of Stim-Tdl On/Off flies normalized with respect to the TAG equivalent content/protein content of day 1 Stim-Tdl Off flies in dllp2-3,5 heterozygous (serving as reference). Note that the obesity phenotype develops after StimTransient drug-feeding induced RNAi expression (Stim-Tdl On) in homozygote dllp2-3,5 mutant, albeit the fold increase was smaller than in Stim-Tdl On dllp2-3,5 heterozygous mutants. For details of genetic constitution of flies and experiments see 2.1.1, 2.1.2, 2.1.5, 2.1.7, 2.3.1, 2.3.3. Stim RNAi1 transgene line was used in following studies. Error bars represent the standard deviation of samples. ${ }^{*}=p<0.05,{ }^{* *} ;=p<0.01,{ }^{* * *}=p<0.001$ (Student's t-test). 
It is important to note that loss of function of insulin peptide genes dllp2,3,5 did not prevent abnormal fat accumulation in Stim-Tdl On flies, which had a similar 2-fold increase in body fat content as Stim-Tdl On in flies which were dllp2-3,5 heterozygous (Figure 16H). Moreover, Stim-Tdl On also led to LISO in dFOXO loss-of-function mutations (Figure 26 (Supplementary data) G). These results indicate that the longterm body fat storage increase in LISO flies is at least partially independent of insulin signaling.

Taken together, these data suggest that the long-term impairment of STIM function in response to Stim-Ttl is very likely to (i) increase the expression of positive regulators gene of insulin signalling; (ii) to reduce the expression of negative regulators of insulin signalling negative regulator genes, and (iii) promotes insulin peptide secretion from IPCs, and (iv) induces continuous body fat storage independently from insulin resistance.

\subsubsection{The expression of lipolysis and $\beta$-oxidation genes is reduced in LISO flies} Regarding energy expenditure and storage, I next asked why LISO flies store excessive fat regardless of possible insulin resistance. Since ${ }^{14} \mathrm{C}$ labeling lipid incorporation assay indicate the up-regulation of lipid biosynthesis (Figure 12E, F) and RNA-seq data indicate the substantial down-regulation of many metabolismrelated genes, especially lipolysis and $\beta$-oxidation related genes (Figure 17A), I turned to investigate whether lipid utilization and lipid synthesis were inhibited after Stim-Ttl.

In fly, the lipase encoded by brummer $(\mathrm{bmm})$ is a homolog of human adipocyte triglyceride lipase (ATGL) (Grönke et al., 2005). Based on RT-qPCR results from fat tissue samples (Figure 17B), there was an observable down-regulation trend of bmm gene expression at day 0 , albeit this decrease is not statistical significant. However, there was a significant down-regulation of bmm expression (about $37 \%$ ) in Stim-Ttl On male flies at day 10 . This observation is consistent with previous findings (Baumbach et al. 2014b). 
A
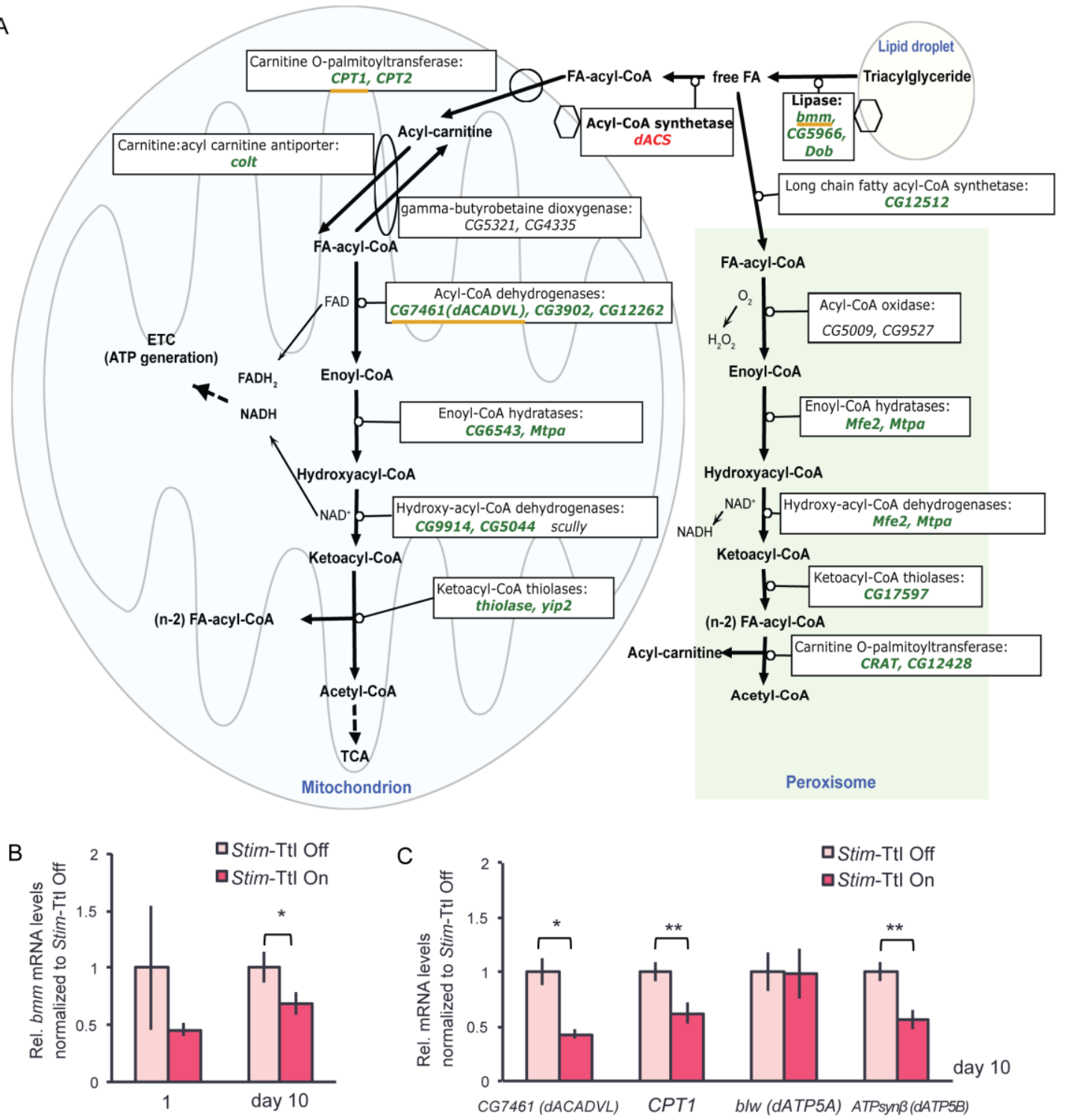

Figure 17. mRNA expressions of the genes involved in lipolysis and $\beta$-oxidation were reduced in LISO fly fat body tissue

A. Schematic representation of the biochemical pathways of lipolysis and $\beta$-oxidation, their localization within the cells, i.e. mitochondria, cytoplasm or peroxisomes and the genes that are affected at day 10 after the Stim-TdI On as revealed by RNA-seq analysis. The names of metabolites (black) are marked in bold, enzymes are in boxes; the names of down-regulated genes (bold and italic) are indicated in green; the names of up-regulated gene (bold and italic) in red; and the names of genes that are not affected are indicated in black and italic. FA: fatty acid, ETC: electron transport chain on mitochondria membrane involved in the generation of ATP by using reduced form of Nicotinamide adenine dinucleotide (NADH), and reduced form of flavin adenine dinucleotide (FADH2); TCA: tricarboxylic acid cycle, a series of biochemical reactions get involved in generation of reducing reagent $\mathrm{NADH}$. The scheme was made with PathVisio (https://www.pathvisio.org/), and the metabolism knowledge were integrated from flybase (http://flybase.org/), wikipathways (http://www.wikipathways.org), reactome (http://www.reactome.org), and (Faust et al., 2012). The down-regulation of the genes with orange underlining was confirmed by RT-qPCR. B.C. RT-qPCR analyses were carried out on the fat body tissues of Stim-TtI On and Off adult males. B. The mRNAs expression of the lipolysis gene $\mathrm{bmm}$ was likely reduced at day 0 and significantly reduced at day 10 after Stim- 
Ttl On as compared to corresponding control (Stim-Ttl Off) flies. C. The mRNAs expression of CG7461 (dACADVL) and CPT1 (see A for their position in the $\beta$-oxidation pathway) as well as the gene encoding the ATP synthase $\beta$-subunit (ATPsyn $\beta)$ are reduced at day 10 after StimTtl On as compared to corresponding control (Stim-Ttl Off) flies, whereas expression of bellwether (bwl), which encodes the ATP synthase subunit $\alpha$, is not affected. Relative (Rel.) mRNA levels are represented as fold change, which refers to the basis value $(=1)$ obtained from Stim-Ttl Off flies; Act5C mRNA served an internal standard and was used as reference to normalize the levels of above mentioned gene mRNA expression. Stim RNAi1 transgene line was used in following studies. Error bars represent the standard deviation of samples. * $=p<0.05,{ }^{* *} ;=p<0.01,{ }^{* * *}=p<0.001$ (Student's t-test). For details of RT-qPCR see 2.2.4.1.

Conversely, the mRNA of lipid biosynthesis gene mdy, which encodes the fly homolog of human diacylglycerol acyltransferase (DGAT) (Buszczak et al., 2002) showed a significant increase at day 0, but not at day 10 in Stim-TtI On flies as compared to the controls (Figure 26 (Supplementary data) C). Similarly, I observed no difference with respect to the expression of other genes (fatty acid synthase gene $d F A S$, Acetyl Coenzyme A synthase gene dACS, $\mathrm{Mg}^{2+}$-dependent PA phosphatase gene dLipin, DGAT gene $m d y$, lipid synthesis transcription factor sugarbabe gene sug, involved in lipid biosynthesis at day 10, except transformation growth factor $\beta$ family Activin-like ligand Dawdle daw (Mattila et al., 2015) was transcriptionally upregulated. (Figure 26 (Supplementary data) D). Moreover, compared to day 10 Stim-Ttl Off flies, the expression of the Acyl-CoA dehydrogenase gene CG7461, encoding a fly homologue of human Acyl-CoA Dehydrogenase, Very Long chain (ACADVL), was decreased by $57 \%$ in day 10 Stim-TtI On flies (Figure 17C). The down regulation (by 38\%) of CPT1 mRNA, which encodes carnitine palmitoyltransferase 1 (CPT1), was validated at day 10 after Stim-TtI On (Figure 17C). CPT1 is responsible for converting fatty acid acyl CoA into Acyl-carnitine, a substrate of $\beta$-oxidation in mitochondria. In addition, a $43 \%$ decrease of the expression of the gene ATPsyn $\beta$ encoding the $\beta$ subunit of ATP synthase was noticed at day 10 , but the alpha unit of the ATP synthase (ATP5A) gene bellwether (b/w), was not differentially expressed (Figure 17C).

In summary, the Stim-TtI On is accompanied by a down-regulation of genes involved in lipolysis and mitochondrial $\beta$-oxidation and normal expression of genes involved in lipid biosynthesis. These results indicate that lipid mobilization and ATP generation were likely impaired during LISO development. 


\subsubsection{The expression of malate/aspartate shuttling genes is reduced in LISO}

flies

Next, I decided to search for a link between calcium signalling, the regulation of lipolysis and mitochondrial $\beta$-oxidation from metabolic control to transcriptional regulation.

RNA-seq data revealed a significant decrease of gene activity of all malate/aspartate shuttle genes in fat body tissues of day 10 Stim-Ttl On flies (Figure 18A), which were confirmed by RT-qPCR. The levels of mRNA of arlar1 was reduced by $30 \%$, Mdh1 by $82 \%$, Mdh2 by $54 \%$, Got1 by $46 \%$, and Got2 by $64 \%$ (Figure 18B). Moreover, to determine if these alterations in gene expression started soon after after Stim-Ttl, I examined the expression of the genes also in day 1 Stim-TtI On and Stim-Ttl Off flies. The results show that the expression of Mdh1 was reduced by $70 \%$ in the Stim-Ttl On flies (Figure 18C). These results further support the argument that transcription repression of aspartate/malatate shuttling genes might also contribute to the development of LISO.

I finally asked how the calcium levels might regulate Mdh1 expression. In yeast, it was found that the calcium-dependent phosphatase calcineurin directly regulates expression of $M D H 2$, and likely $M D H 1$ through calcineurin responsive transcription factors (Ruiz et al., 2008). In line with this observation, I found that the gene encoding the Drosophila calcineurin CanA-14F was significantly down-regulated by nearly $30 \%$ in day $10 \mathrm{Stim}$-TtI On flies as compared to the corresponding control flies (Figure 18D). Moreover, the expression of $M D H 1$ was regulated by PGC-1 $\alpha$ in mice muscle (Handschin et al., 2007), the nuclear hormone receptor the mammalian PGC$1 \alpha$ homolog dPGC-1 was also significantly down-regulated at day 10 Stim-TtI On flies as compared to the corresponding control flies (Figure 18E). It has been reported that mitochondrial aspartate/glutamate carrier protein encoded by aralar1, the single homolog of human SLC25A12 or 13 in fly, can be activated by calcium level (Del Arco et al., 2000). Aralar1 is essential for malate/aspartate shuttle (Contreras et al., 2007; Lasorsa et al., 2003; Palmieri et al., 2001). Given the longterm impairment of calcium signalling in Stim-Ttl flies, it is reasonable to assume that the Aralar1 carrier function might be also affected. 


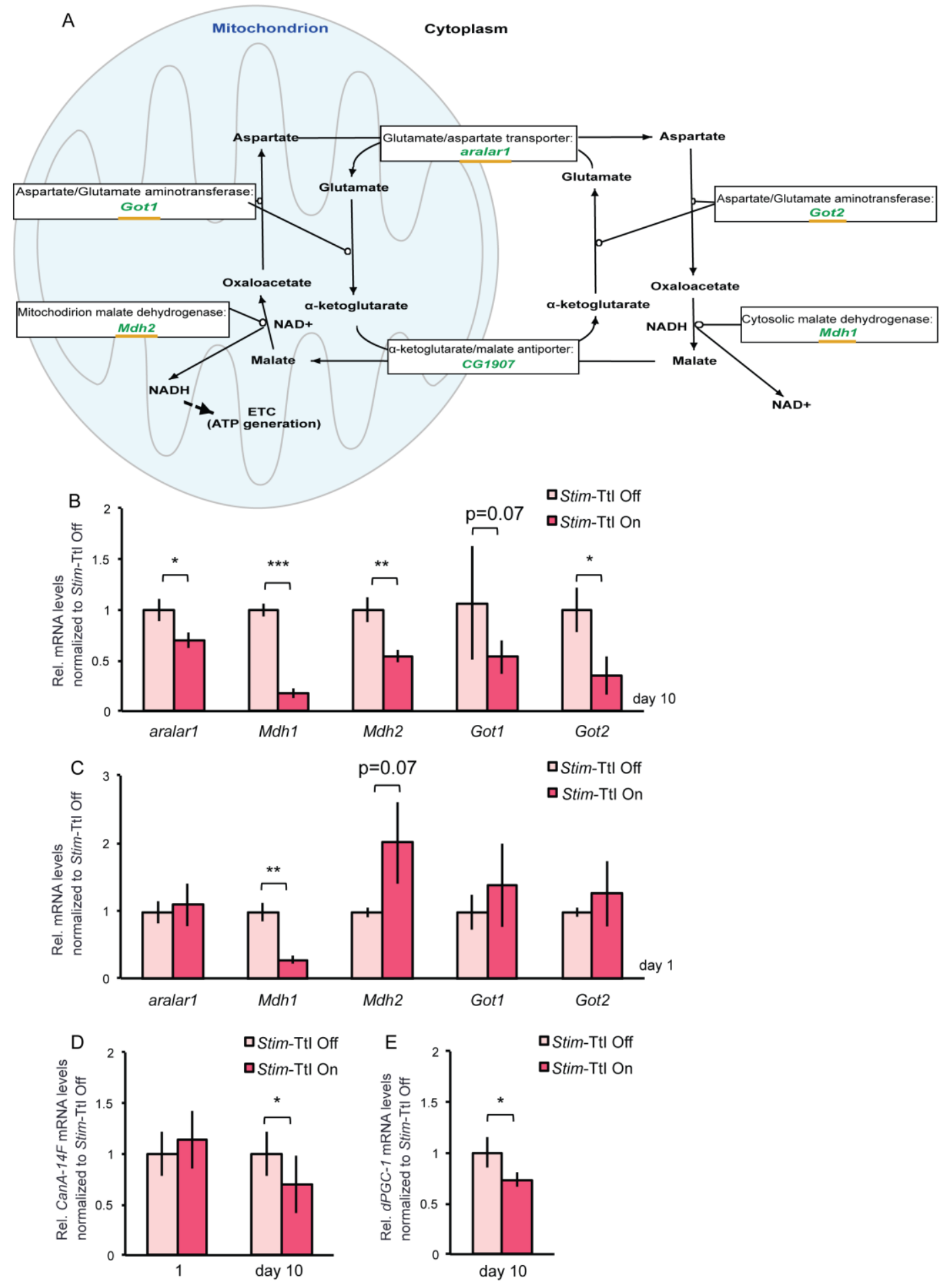

Figure 18. mRNA expressions of the genes encoding enzymes participating in malateaspartate shuttle between cytoplasm and mitochondria were reduced in LISO fly fat body tissue

A. Schematic representation of enzymes of the biochemical pathways which shuttle malateaspartate between cytoplasm and mitochondria, which is normally transporting of reducing reagent NADH from cytoplasm to mitochondria ("malate-aspartate shuttle"). The names of metabolites (black) are marked in bold, enzymes are in boxes; the names of down-regulated genes (bold and italic) are indicated in green; the names of up-regulated gene (bold and italic) in red; and the names of genes that are not affected are indicated in black and italic. The scheme was made with PathVisio (https://www.pathvisio.org/), and the metabolism 
knowledge were integrated from flybase (http://flybase.org/), wikipathways (http://www.wikipathways.org), reactome (http://www.reactome.org), and (Palmieri et al., 2001). The down-regulation of the gene with orange underlining was confirmed by RT-qPCR.

B. C. D. E. RT-qPCR analysis on the mRNAs expressions of day 10 and day 1 Stim-Ttl On flies fat body tissue as compared to the corresponding control (Stim-Ttl Off) flies. B. At day 10, all the malate-aspartate shuttle genes aralar1, Mdh1, Mdh2, Got1 and Got2 were significantly down-regulated except Got1, although Got1 also showed a downward trend. C. However, at day 1 , only the mRNAs expression of $M d h 1$ is already down-regulated, whereas the expression of the other genes is not significantly affected at day 1 after Stim-Ttl On as compared to the corresponding control (Stim-Ttl Off) flies.

D. The mRNA expression of gene encoding the calcium effector calcineurin subunit CanA$14 \mathrm{~F}$ is not affected at day 1 after Stim-Ttl On as compared to the corresponding control (StimTtl Off) flies, but significantly down-regulated at day 10 after the induction time point. (E) The mRNA expression of gene encoding the nuclear receptor dPGC-1 was significantly downregulated in day $10 \mathrm{Stim}$-TtI On adult male fat body tissues as compared to Stim-Ttl Off flies. Relative (Rel.) mRNA levels are represented as fold change, which refers to the basis value $(=1)$ obtained from Stim-Ttl Off flies; Act5C mRNA served an internal standard and was used as reference to normalize the levels of above mentioned gene mRNA expression. Stim RNAi1 transgene line was used in following studies. Error bars represent the standar deviation of samples. ${ }^{*}=p<0.05,{ }^{* *} ;=p<0.01,{ }^{* * *}=p<0.001$ (Student's t-test). For details of RT-qPCR see 2.2.4.1.

In summary, these findings establish that impaired calcium signalling due to Stim-TtI affect malate/aspartate shuttle genes, and it might involve calcineurin and dPCC-1 mediated transcription programs. 


\section{Discussion}

The conservation of energy homeostasis in Drosophila melanogaster offers an excellent model for better understanding of tissue-specific genetic risk factors causing energy imbalance. In addition, studies in Drosophila obesity research provide deep insights into how the dysfunction of a single organ results in the collapse of the entire, multilevel energy homeostasis regulatory network leading to obesity. In this study, I have investigated the causal link between impairing the calcium signalling pathway and obesity development in adult flies. I have experimentally reduced calcium signalling specifically in the fat storage tissue by transient Stim RNAi expression. The fat body in Drosophila is the representative organ of fat storage tissue. The results indicate that this impairment causes an increase of the fat content of the fly. The findings also provide evidence that adipokinetic hormone (Akh) signalling mobilizes lipids in the wild type fly at least partially by AkhR (GPCR)/G protein/PIc $\beta / d S T I M$ signal transduction. I also identified and characterized the "Long-term Impairment of STIM function in fat storage tissue Induced Obesity (t-LISO or d-LISO), which occurs in response to the "StimTransient temperature-shift Induced or drug-feeding Induced Stim RNA interference (Stim-Ttl, or TtI)". Regarding t-LISO or d-LISO from Stim-Ttl, or Ttl, which I will also refer in the following discussion simply and collectively as LISO in response to StimTI. My data suggest that Stim-TI provokes a typical positive energy imbalance pattern such as increased food intake, reduced locomotor activity and a diminished metabolic rate. Moreover, my study indicates that Stim-TI results in increased lipid biosynthesis but reduced expression of lipolysis and fatty acid $\beta$-oxidation genes. Moreover, Stim-TI promotes hyperphagia likely via a remote up-regulation of Akh secretion from the corpora cardiaca. Finally, the study also suggests that LISO development involves peripheral insulin resistance and fat body tissue Akh signalling resistance. These findings provide new insights into the mechanisms of obesity induced by calcium signalling dysfunctions, expand our view of the energy homeostasis regulatory network, and further establish Drosophila adult flies as an attractive model system for integrative physiological studies of metabolic disorders.

\subsection{Akh/AkhR signalling regulates fat storage via GY1-Gaq49B- PIc21C-Stim modulated $\mathrm{Ca}^{2+}$ level changes}

I examined cellular lipid droplets accumulation with fat cell staining and confocal imaging to show that functional in vivo impairment of potential Stim upstream factors 
in fat body tissue, encoded by genes such as Plc21C, Gy1, Gaq49B and AkhR, promotes excessive fat accumulation in adult virgin female fat body tissue. This effect was also observed in $A k h R$ loss-of-function mutants as well as in flies with $A k h$ knockdown in the corpora cardiaca cells. These findings are consistent with body fat content quantification results obtained by enzymatic assay (Baumbach et al., 2014a) (Baumbach et al., 2014b), neutral lipids separation analysis by thin layer chromatography (TLC), and Oil Red O staining on adult male flies (Baumbach et al., 2014b). The results also confirm previous findings that inhibiting AKH or AkhR signalling increases body fat storage (Bharucha et al., 2008; Gáliková et al., 2015; Grönke et al., 2007). On the other hand, over-expression of $A k h, A k h R$ in fat body tissue causes reduced cellular lipids droplets accumulation, which are therefore in line with the long known role played by Akh/AkhR signalling in promoting lipid mobilization in different insect species (Van der Horst et al., 2001) and more recent observations in Drosophila (Gáliková et al., 2017; Grönke et al., 2007; Lee and Park, 2004). Previous studies have shown that incubating insect fat body with Akh induces significant, dose- and time-dependent production of inositol $(1,4,5)$ triphosphate (IP3) (Van Marrewijk et al., 1996; Vroemen et al., 1997). In addition, it has been established that $G$ protein $\alpha q$ interacts with Phospholipase $C \beta$ (PLC $\beta$ ) to produce secondary messengers of IP3 and diacylglycerol (DAG) in mammalian cells as well as in Drosophila tissues (Deng et al., 2015; Hepler and Gilman, 1992; Kain et al., 2008; Sternweis and Smrcka, 1992). The cytosolic IP3 activates IP3 receptors (IP3Rs), which initiates store-operated calcium entry (SOCE), causes the elevation of cytosolic $\mathrm{Ca}^{2+}$ levels (Berridge, 2009; Soboloff et al., 2012). Moreover, Akh mediated lipolysis in $M$. sexta also gets involved in rapid $\mathrm{Ca}^{2+}$ influx (Arrese et al., 1999). Collectively, these results support the possibility that Stim, Plc21C, Gy1, Gaq49B, $A k h R$, and $A k h$ may act together in the same pathway to regulate downstream fat metabolism.

Indeed, similar to Stim in vivo knockdown (Baumbach et al., 2014a,b), in vivo impairment of Stim potential upstream factor gene activities such as Plc21C, Gy1, Gaq49B and $A k h R$ in fat body tissue led to a reduced cytosolic $\mathrm{Ca}^{2+}$ level indicated by calcium responsive membrane GFPs. Further quantification confirmed the positive correlation between gene activities of Stim, Plc21C, Gy1, Gaq49B, AkhR and the cytosolic $\mathrm{Ca}^{2+}$ level (Baumbach et al., 2014b). These findings are in line with results from extensive studies in other insects (reviewed in Gäde and Auerswald, 2003), which support the conclusion that the regulation of lipid mobilization in response to Akh/AkhR signalling requires cytosolic calcium signalling. For instance, removing 
extracellular calcium by calcium chelators prevented the response of the fat body to Akh in S. gregaria (Spencer and Candy, 1976), L. migratoria (Vroemen et al., 1995) as well as in M. sexta (Arrese et al., 1999). Moreover, extracellular calcium influx was observed after endogenous Akh binding to its receptors in L. migratoria (Vroemen et al., 1995). Consistently, blocking calcium channels with drugs such as verapamil and nifedipine reduce the effect of Akh on lipid mobilization (Arrese et al., 1999). Conversely, increasing the calcium release from endoplasmic reticulum (ER) stores by inhibiting the ER $\mathrm{Ca}^{2+}$-ATPase (SERCA) stimulated the lipid mobilization from the M. sexta fat body (Arrese et al., 1999). Similar positive effects on lipid mobilization were also found after injecting $\mathrm{Ca}^{2+}$ ionophores in adult L. migratoria (Lum and Chino, 1990) or M. sexta (Arrese et al., 1999). Taken together, the activity of Plc21C, Gy1, Gaq49B and $A k h R$ very likely regulate fat storage in Drosophila via Calcium signalling, and this control of fat storage is common to insects in general.

A well-known fact concerning Akh/AkhR-mediated lipid mobilization is its dependence on the Gs protein/adenylyl cyclase (AC)/cAMP/protein kinase A (PKA) signalling pathway in many insects and in Drosophila (Gäde and Auerswald, 2003; Grönke et al., 2007). More specifically, in M. sexta, Akh may induce activation of PKA by phosphorylation, which in turn causes an increase of triaclyglyeride (TAG) lipase activity (Arrese and Wells, 1994; Patel et al., 2005). Moreover, PKA can phosphorylate the lipid droplet (LD) localized protein perilipin 1 (PLIN1) of Drosophila, which likely makes LDs more easily accessible for TAG lipase as shown for M. sexta (Arrese et al., 2008; Patel et al., 2005). Similarly, Drosophila PLIN1 also plays a key role in stimulating lipolysis by adjusting the access of proteins to LDs surface (Beller et al., 2010). For example, PLIN1 is required for the translocation of another key lipase, the hormone-sensitive lipase (HSL), from cytoplasm to LDs as shown for Drosophila larvae under starvation (Bi et al., 2012). Interestingly, in mammals, in response to Gs protein-coupled receptor-dependent PKA signalling, which is initiated in response to $\beta$-adrenergic receptor or glucagon receptor activities, the phosphorylation of PLIN1 triggers the release of lipase activator protein CGI-58 (also called $\alpha / \beta$-hydrolase domain containing 5, ABHD5) (Lass et al., 2011; Zechner et al., 2009). This in turn further interacts with adipose triacylglyceride lipase (ATGL) and stimulates the lipase activity of ATGL (Lass et al., 2011; Zechner et al., 2009). As in mammals, PLIN1 is essential for the translocation of HSL to the LDs; PKAmediated phosphorylation of the hormone-sensitive lipase (HSL) induces the intrinsic enzymatic activity of HSL, and phosphorylated PLIN1 facilitates extra lipolysis activity of HSL (Zechner et al., 2009). More importantly, post-translational regulation of lipid 
mobilization by Gs protein/AC)/PKA signalling is also dependent on the intracellular $\mathrm{Ca}^{2+}$ level (Arrese et al., 1999; Wang et al., 1990). The crosstalk between $\mathrm{Ca}^{2+}$ and ACs has been identified in mammals, which leads to either AC inhibition (AC6) or activation (AC1, AC3, and AC8) by $\mathrm{Ca}^{2+}$ (Cooper, 2015). For example, cAMP production from $\mathrm{AC} 8$ can be directly induced by $\mathrm{Ca}^{2+}$ influx (Willoughby and Cooper, 2006). Moreover, a direct interaction between AC8 and store-operated calcium entry (SOCE) calcium channel protein ORAl1 has been identified (Willoughby et al., 2012), and it has been shown that CAMP production, CAMP-dependent HSL phosphorylation and lipolysis were impaired in ORAl1 and SOCE mutant fibroblasts (Maus et al., 2017). Intriguingly, the Drosophila genome encodes a mammalian homolog of AC1, called rutabaga, which is stimulated by $\mathrm{Ca}^{2+} /$ Calmodulin (Levin et al., 1992). A mutation of the rutabaga gene has been linked to increased fly TAG stores (JumboLucioni et al., 2010). Given that Akh is functionally homologous to glucagondependent signalling in mammals (Droujinine and Perrimon, 2016; Kim and Rulifson, 2004), AkhR, Gaq49B, Gy1, Plc21, and Stim are likely to control lipid mobilization via $\mathrm{Ca}^{2+} /$ rutagaba/cAMP crosstalk mediated post-translational regulation.

Our studies also uncovered transcriptional alterations of lipid biosynthesis and lipolysis genes. In fact, in vivo acute functional impairment of Stim and of the activities of its potential upstream acting genes $A k h R, G \alpha q 49 B, G \gamma 1, P I c 21 C$ in fat body tissue of males increased the transcription of lipid biosynthesis gene $m d y$ (Baumbach, Xu et al., 2014b), which is homologous to mammalian gene that encodes Acyl-CoA: diacylglycerol acyltransferase (DGAT). Hypomorphic mdy lossof-function mutants develop into lean flies (Beller et al., 2010). It has been reported that the cAMP-responsive transcription factor CREB promotes energy utilization in the mammalian liver and adipose tissues in response to $\beta$-adrenergic or glucagon signalling (Altarejos and Montminy, 2011). The Drosophila homolog of mammalian CREB is called CrebB; inhibiting CrebB activity by over-expression of a dominant negative form in fat body tissue increased the body fat content of the fly (Baumbach, 2014; lijima et al., 2009). Moreover, CrebB-mediated transcription indicated by CRELuciferase reporter transgene expression is decreased in response to AkhR impairment (lijima et al., 2009). Reduction of CrebB activity, in turn, increased the transcription of lipogenic gene $m d y$ and down-regulated the transcription of lipase gene bmm (Baumbach, 2014). These observations are consistent with my results where $m d y$ increased expression in response to Stim RNAi treatment and the finding that simultaneous knockdown of Stim under Akh overexpression condition not only restored the body fat depletion normally caused by Akh signalling, but also further 
increased the body fat content (Baumbach et al., 2014b). Similarly, knockdown experiments involving Gy1, Plc21C, or Gaq49B in combination with Akh overexpression also restored the body fat depletion to a similar or even slightly higher level than observed with the corresponding controls (Baumbach et al., 2014b). Taking these epistasis results into account, it appears that our findings link the candidate AkhR, Gaq49B/Gy1/PIc21C/Stim pathway to transcriptional expression of the key lipid biosynthesis gene by CrebB.

The expression of the lipolysis gene $b m m$, which encodes the major Drosophila TAG lipase (Grönke et al., 2005), was also affected in flies expressing Gaq49B, Gy1, Plc21, Stim RNAi (Baumbach et al., 2014b). Unlike the functional impairment of Gaq49B, Gy1, Plc21 and Stim gene activities, the corresponding impairment of $A k h R$ in fat body tissue did not repress the expression of $b m m$, which is in line with the results of genetic interaction experiments: the Stim RNAi knockdown in fat body tissue caused the lipolysis effect beyond the response to Akh and caused extra fat accumulation (Baumbach et al., 2014b). This result also corroborates the finding that Akh/AKHR/cAMP/PKA-dependent rapid lipolysis may distinct from the basal and extended starvation-induced lipolysis mediated by bmm (Grönke et al., 2007). These findings may also indicate that the Gaq49B-Gy1-PIc21C-Stim module not only transmits Akh/AKHR signalling, but possibly also integrates the stimuli from other membrane receptors. For example, null mutant of Bride of sevenless (BOSS) gene, encoding a G protein-coupled receptor (GPCR) found in fat body tissues, caused abnormal sugar and lipid metabolism in fly larvae (Kohyama-Koganeya et al., 2008). It would be interesting to investigate whether BOSS can modulate Gaq49B-Gy1Plc21C-Stim to affect fat storage in adult fly adipose tissue.

\subsection{LISO in adult Drosophila as an obesity model}

\subsubsection{Transient Stim RNAi expression in fat body promotes long-term fat storage increase}

The conserved role of STIM in raising cytosolic $\mathrm{Ca}^{2+}$ level has been established by RNAi-mediated knockdowns of Stim in Drosophila S2 cells and by STIM1 in mammalian cells where SOCE provoked by the drug thapsigargin was significantly reduced (Roos et al., 2005). Here, I established the temperature-shift inducible StimRNAi system, which enables efficient expression or repression of double-stranded RNA (dsRNA) from Stim RNAi transgene under Stim-Ttl On and Stim-Ttl Off 
conditions in fat storage tissue, respectively. Similarly, a drug-inducible Stim-RNAi knockdown system, Stim-Tdl, was established. After Stim-Ttl in whole flies, Stim mRNA expression was not only reduced by around $50 \%$ at day 0 , but also remained at low level at day 10 after Stim-Ttl On or at day 8 after Stim-TdI On. These results indicate a long-term functional impairment of dSTIM. These results are also consistent with previous findings showing that both dSTIM protein and cytosolic $\mathrm{Ca}^{2+}$ levels were significantly reduced after a 6 days acute knockdown of Stim in the fat storage tissue of fly males (Baumbach et al., 2014a).
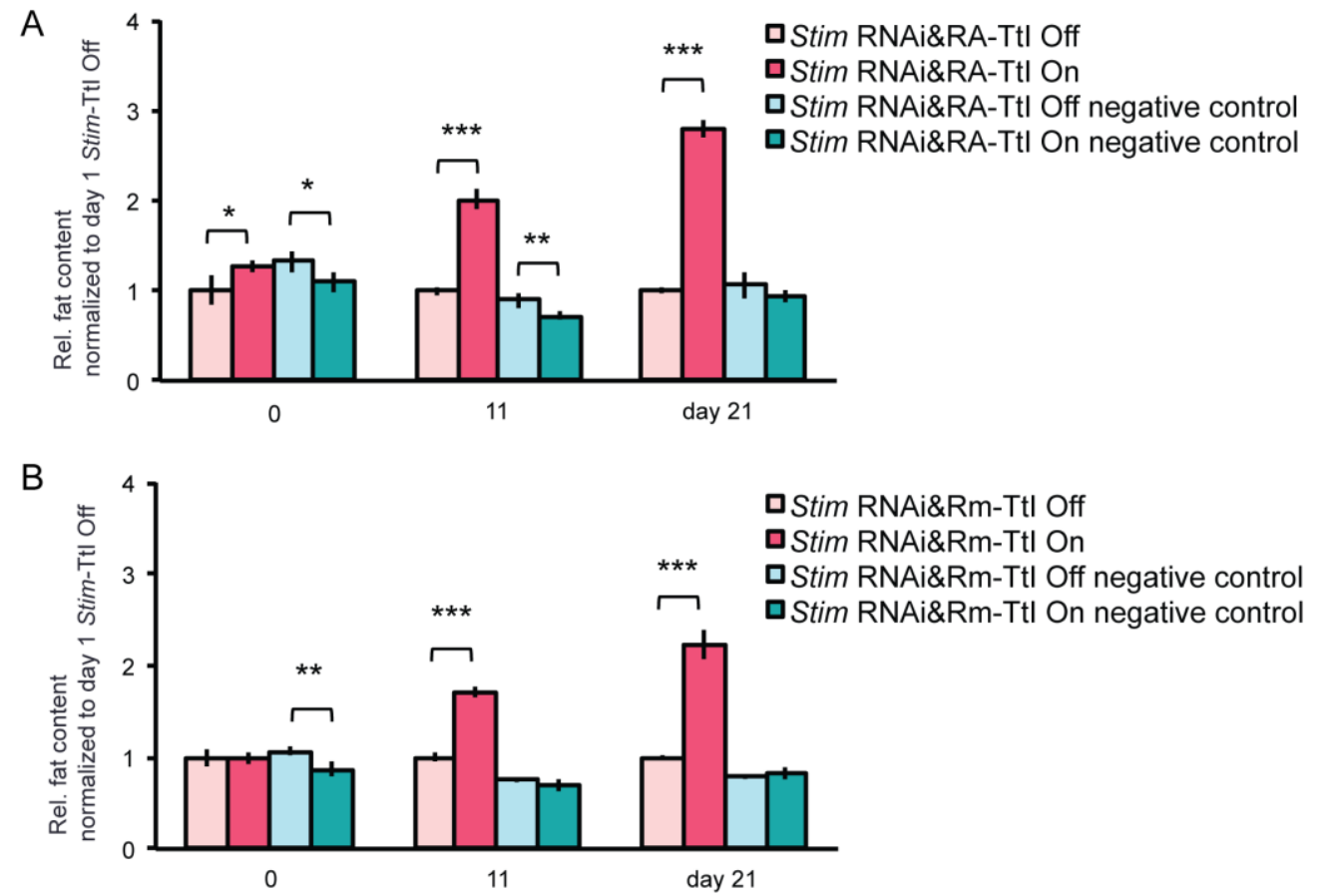

Figure 19. (Preliminary data) Co-expression of Stim RNAi and RNAi resistant cDNA does not rescue the long-term body fat storage increase

A. Quantification of the body fat contents on day 0 , day 11 and days 21 Stim-Transient temperature-shift Induced RNAi expression \& cDNA RA overexpression for $38 \mathrm{~h}$ (StimRNAi\&RA Ttl On) in adult male flies, and on corresponding control (Stim RNAi\&RA -Ttl Off, Stim RNAi\&RA-Ttl On negative control, Stim- RNAi\&RA Ttl Off negative control) flies.

B. Quantification of the body fat contents on day 0, day 11 and days 21 after Stim-Ttl \& cDNA RA modified RNAi resistant construct overexpression for $38 \mathrm{~h}$ (Stim-RNAi\&Rm Ttl On) in adult male and female flies, and on corresponding control (Stim-RNAi\&Rm-Ttl Off, Stim RNAi\&Rm-Ttl On negative control, Stim- RNAi\&Rm-Ttl Off negative control) flies. Unlike Stim RNAi\&RA-Ttl On, Stim RNAi\&Rm-Ttl On did not cause primary increase of body fat content at day 0 compared to corresponding control flies. Both Stim-RNAi\&RA Ttl On and Stim RNAi\&Rm-Ttl On cause the long-term body fat content increase at day 11 and 21 . For details on the nomenclature of Ttl On, Ttl Off, Ttl On negative control, Ttl Off negative control see Figure 3. Relative (rel.) fat content is represented as fold change: TAG content/protein content of Stim-Ttl On flies normalized with respect to the TAG content/protein content of Stim-Ttl Off flies. Stim RNAi1 transgene line was used in above studies. Stim RNAi1 transgene line was used in these studies. Error bars represent the Standard Deviation of 
samples. ${ }^{*}=p<0.05,{ }^{* *}=p<0.01,{ }^{* * *}=p<0.001$ (student t-test). Only one round experiment was done. For details of genetic constitution of flies and experiments see 2.1.1, 2.1.2, 2.1.6, 2.1.7, 2.2.1, 2.3.1, and 2.3.3.

Following the Stim-Ttl, flies already started to accumulate extra fat as early as at day 0 , and continuously accumulated excessive fat at day 10 and day 21 after the RNAi treatment. It is of notice that the body fat content of negative control flies was moderately and significantly reduced by temperature-associated stress at day 0 but recovered at day 10 . This result is in line with previous results showing that fat depletion occurs after thermal stress (Klepsatel et al., 2016). It indicates that the long-term obesity development is indeed induced by Stim RNAi expression and not due to thermal stress. Furthermore, similar to Stim-Ttl flies, a moderate but significant increase in body fat was also found at day 1 Stim-Tdl flies, which continuously increased their body fat at day 7, and day 15 after Stim RNAi expression. These results exclude the influence of gene-environment interaction as the cause of obesity.

Furthermore, a RNAi off-target effect can also be excluded, as Stim-TI provided by a different Stim RNAi transgene that target different sequences of Stim mRNA also cause long-term body fat increase. In order to show the causal role of Stim-Ttl in fat accumulation, I induced simultaneous expression of Stim cDNA RA (Stim RNAi sensitive cDNA, RA), or Stim cDNA RA modified RNAi resistant transgene (Stim RNAi resistant cDNA, Rm) and Stim RNAi dsRNA transgene in fat storage tissue. Stim RNAi expression was still able to provoke a $27 \%$ increase of the body fat content at day 0 despite the concomitant overexpression of Stim RNAi sensitive cDNA-RA transgene (Figure 19 (Preliminary data) A). However, expression of Stim RNAi resistant cDNA suppressed the primary body fat content in response to StimRNAi at day 0 (Figure 19 (Preliminary data) B). However, these preliminary findings are consistent with the idea that it is the impairment of dSTIM function rather than RNAi off target effects that increased fat storage in adult males. Additionally, it was found that hypomorphic mutations of inositol 1,4,5-trisphosphate receptor (InsP3R) decrease the cytosolic $\mathrm{Ca}^{2+}$ levels and cause excessive fat storage in adult Drosophila (Subramanian et al., 2013). While the knockdown of mRNA of the gene that encodes the calcium pump dSERCA increased Drosophila fat body tissues cytosolic $\mathrm{Ca}^{2+}$ levels, which diminished fat storage (Bi et al., 2014a). Moreover, the connection between functional impairment of STIM and increased fat storage described here for Drosophila was confirmed in mammals where excessive fat was 
stored in the liver, heart and skeletal muscle cells of SOCE-deficient mice as well as in fibroblasts from patients carrying a loss-of-function mutations in STIM1 or ORAI1 (Maus et al., 2017).

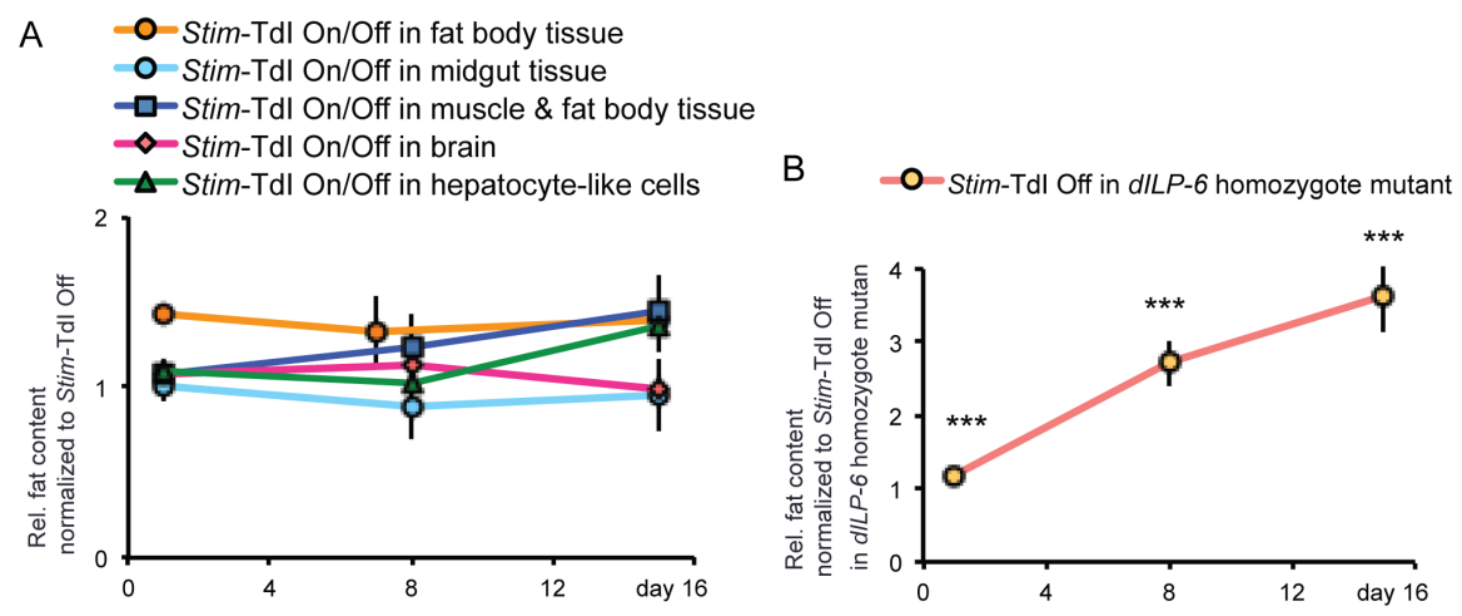

Figure 20. (Preliminary data) Body fat content changes of flies with Stim-Tdl in different organ tissues of adult male flies, or in $d I I p-6$ candidate mutant

A.B. Quantification of fly body fat contents at day 1,7 or 8 , and 15 after 4 days Stim-Tdl On in different tissue of adult male flies, and corresponding control (Stim-Tdl Off) flies.

A. Stim-Tdl On with FBI-26-GS-Gal4 in fat body tissue (in orange, $p<0.01$ ), or with GSG8708Gal4 in muscle \& fat body tissues (in deep blue, $p<0.001$ ), showed the long-term increased body fat storage at day 7 , and 15 as compared to corresponding control (Stim-Tdl Off) flies. However, Stim-Tdl On driven with TIGS-Gal4 in midgut tissue (light blue), or with ElavGSGal4 in the brain (in light violet) did not cause significant increased body fat content. Stim-Tdl On by promE800GS-Gal4 in oenocyte (Drosophila hepatocyte-like cell) appeared to cause later fat storage increase at day $15(p<0.05)$. B. Stim-Tdl On with daGS-Gal4 in homozygous dllp6 mutant male flies caused the long-term body fat storage increase as compared to corresponding control (Stim-Tdl Off in homozygote dllp6 mutant) flies.

Relative (rel.) fat content is represented as fold change: TAG content/protein content of StimTdl On flies normalized with respect to the TAG content/protein content of Stim-Tdl Off flies. Stim RNAi1 transgene line was used in above studies. For details on the nomenclature of Tdl On, Tdl Off, Tdl On negative control, Tdl Off negative control see Figure 4. Stim RNAi1 transgene line was used in these studies. Error bars represent the Standard Deviation of samples. ${ }^{*}=p<0.05,{ }^{* *}=p<0.01,{ }^{* * *}=p<0.001$ (student t-test). (A) RNAi efficiencies driven by different drivers were not identified yet. (B) dllp-6 loss-of-function mutant was not genotyped yet. For details of genetic constitutions of flies and experiments see 2.1.1, 2,1,2, 2.1.5, 2.3.1, 2.3.3.

It is important to note that the ts-FB-Gal4 driver used in Stim-Ttl drives Stim RNAi expression exclusively in fat storage tissue, i.e. in fat body tissue and in midgut (Baumbach et al., 2014a). Besides the ubiquitous daGS-Gal4 driver normally used (Tricoire et al., 2009), I also tested a number of additional GeneSwitch Gal4 drivers such as the muscle and fat body-specific driver GSG8708-GAL4 (Nicholson et al., 2008) and the fat body-specific driver FBI-GS-GAL4 (Suh et al., 2007) in a series of 
pilot experiments. All these drivers, which caused expression of Stim RNAi in fat body tissue without temperature-shift, increased the body fat content at day 8 and day 15, although to a lower extent than the fat body-specific driver ts-FB-Gal4 (Figure 20 (Preliminary data) A). In contrast, Stim-TI driven by the oenocytespecific driver promE800-GS-Gal4, which causes expression in the hepatocyte-like cells (Chatterjee et al., 2014), the pan neural driver Elav-GS-Gal4 (Osterwalder et al., 2001), or the midgut enterocyte driver TI-GS-Gal4 (Alic et al., 2014) did not cause an increase of the body fat content (Figure 20 (Preliminary data) A). These results suggest that it is the Stim-Ttl or Tdl in fat body tissue that causes the long-term increase of body fat in adult flies. Nevertheless, further studies are needed to done to identify whether the possible dysfunctional interaction between midgut and fat body tissue caused by Stim-TI might also aggravate the obesity phenotype, as a recent study shows that gut derived Activin- $\beta$ induced by high-sugar diet can stimulate Akh signalling (Song et al., 2017).

Collectively, my data support the conclusion that a long-term impairment of STIM function in fat body tissue by Stim- $\mathrm{Tl}$ ( $\mathrm{Ttl}$ or $\mathrm{Tdl}$ ) promotes a long-term increase of body fat storage. This is why I refer to the phenomenon as Long-term Impairment of STIM induced Obesity (LISO).

\subsubsection{LISO is independent of gender and age except in mating female flies}

To test whether LISO development depends on physiological parameters such as age, gender or mating, I performed Stim-Ttl with six and 36 days old flies, in both male and females and in virgin as well as mating females. Obviously, the age difference did not affect LISO development in response to Stim-TtI in adult males and virgin females. Furthermore, the mating process had no significant effect on LISO in adult males. However, mating prevented the LISO development in response to Stim$\mathrm{Ttl}$ in adult females. After mating, insect females like Drosophila continuously produce a number of eggs (Alpatov, 1932). In insects, eggs normally consists of 30$40 \%$ lipids to meet the energetic requirement of embryogenesis, which are maternal provided (Arrese and Soulages, 2010). Previous studies have shown that female flies undergo substantial physiological changes especially in lipid synthesis and trafficking to support the egg production after mating (Parisi et al., 2011; Reiff et al., 2015). In contrast, functional impairment of reproduction causes excessive fat storage not only in Drosophila but also in mammals (reviewed in (Hansen et al., 2013)). The energy cost of reproduction might interfere with Stim-TtI caused body fat 
storage increase, which ultimately causes trade-offs between reproduction and fat storage in female flies. Mating and egg laying female flies may have in fact increased fat synthesis in intestine (Reiff et al., 2015). However this additional fat is not stored in the fat body of the females but stored in the embryo. This assumption needs further studies involving the analysis of the lipid content of the eggs, which are laid by Stim-Ttl females. Nevertheless, it is manifest that Stim-Ttl causes LISO regardless of notable age differences in male and virgin female flies.

\subsubsection{Ttl of putative SOCE upstream genes promotes long-term fat storage increase}

My findings indicate that Ttl of genes which may act upstream of the store-operated calcium entry (SOCE) such as AkhR, Gaq, Gy1 and PIc21C in adult male flies also cause an increased fat storage, although with different temporal profiles (Figure 21 (Preliminary data)). For example, AkhR-Ttl On resulted in an increased body fat storage in day 11 and day 21 male flies. The expression of Gaq-TtI On and Gy1-TtI On resulted in a significantly increased body fat storage at day 21 , but not at day 0 and day 11 (Figure 21 (Preliminary data) A.B). It is of note that in Gy1-Ttl On as well as in the corresponding temperature-shift control male flies (Gy1-TtI On negative control), the body fat storage was reduced at day 0 (Figure 21 (Preliminary data) B). Since the temperature-induced RNAi expression was performed at $29^{\circ} \mathrm{C}$, a temperature which has recently been shown to cause a reduced body fat storage per se (Borcherding, 2014; Klepsatel et al., 2016), I postulate that the temperature associated stress response prevents the extra fat storage during the first 10 days after Gaq or Gy1 RNAi treatment. Nevertheless, Plc21C-Ttl On caused an increase of the body fat storage as early as day 0 , which was further increased at day 11 and day 21 (Figure 21 (Preliminary data) C).

It is also possible that the activities of Gaq or Gy1 transduce different additional receptors' stimuli (Hanlon and Andrew, 2015; Ritter and Hall, 2009) than the other LISO components, which were tested in my study. In addition, a loss-of or reduction of these stimuli in Gaq or Gy1-TtI On flies might then mitigate the onset impact on their fat storage. Plc21C is the more down-stream effector, which is more directly linked to calcium signalling, thereby causing earlier body fat storage increase. Irrespective of the interpretation of these results, the data confirm earlier findings indicating that the expression of $A k h R, G a q 49 b, G y 1$, and Plc21C RNAi in male flies causes the body fat content increase at day 21 (Borcherding, 2014). Moreover, these 
findings are consistent with our in vivo study which provides strong evidence that Akh/AkhR signalling partially promotes lipid mobilization via a cytosolic $\mathrm{Ca}^{2+}$ level change which is modulated in response to the Gaq49/ Gy1/ Plc21C/ dSTIM pathway (Baumbach et al., 2014b).
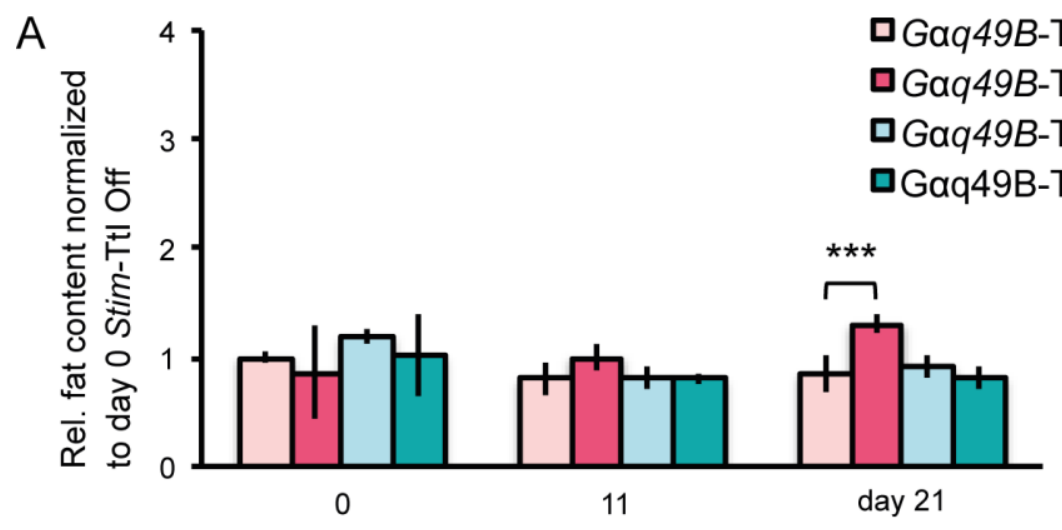

B

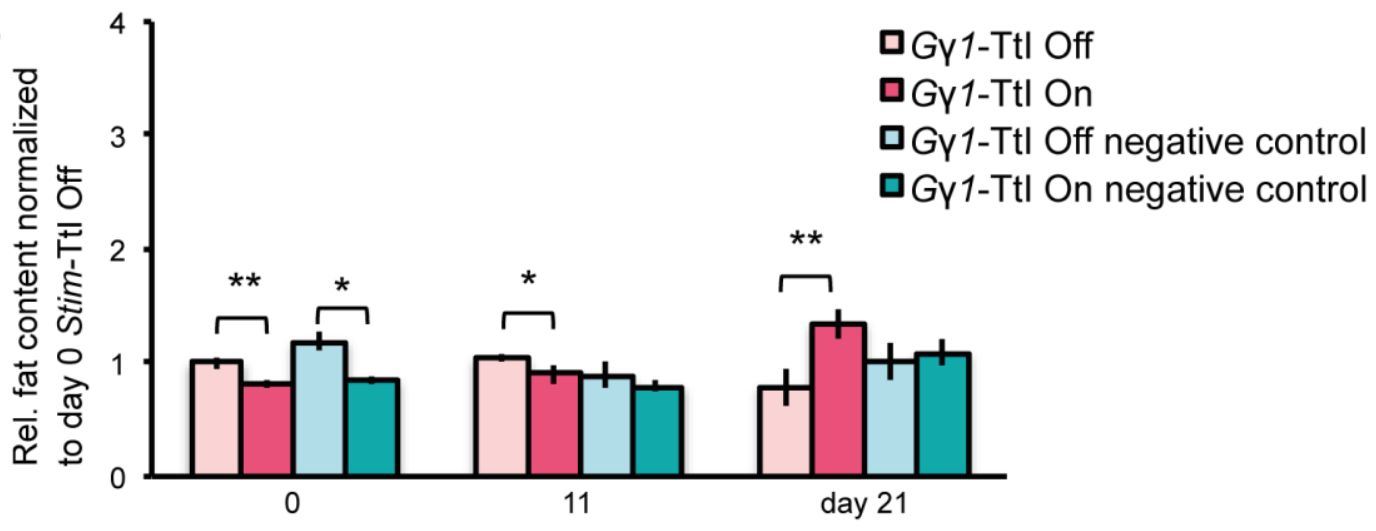

C

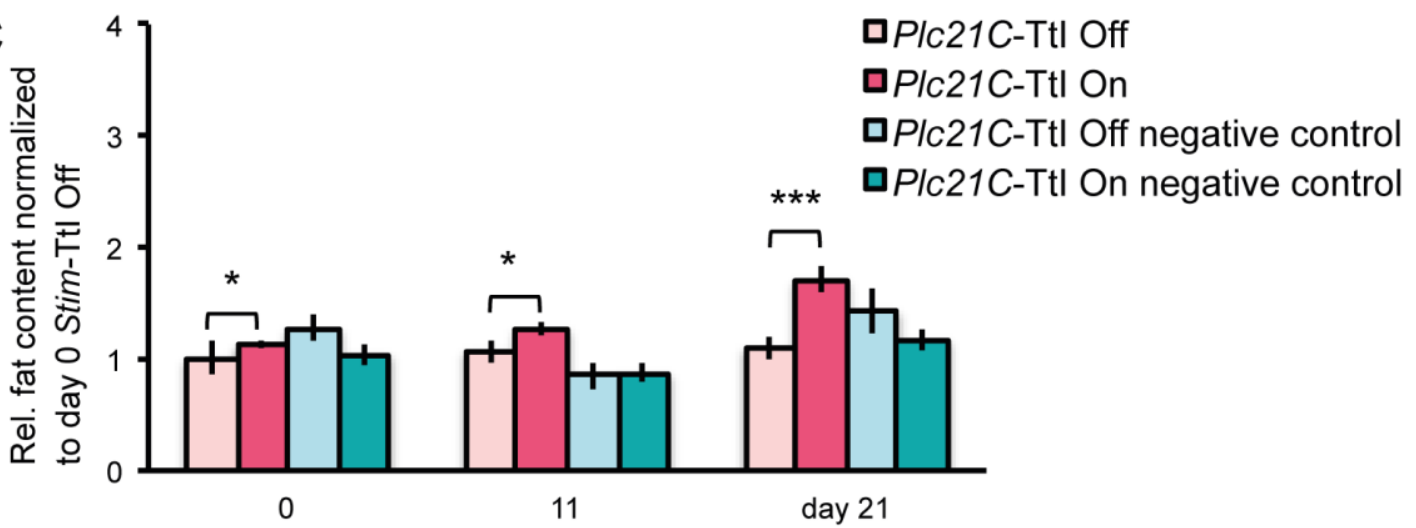

Figure 21. (Preliminary data) Ttl of Stim upstream candidate gene promotes long-term fat storage increase

A. B. C. Quantification of the body fat contents on day 0 or 1,11 and 21 adult male flies after Ttl of Stim upstream candidate genes for 48 hours. AkhR RNAi1, G $\alpha 49 B$ RNAi1, G 11 RNAi1, PIC21C RNAi1 transgenes lines were used in following studies respectively. Relative (rel.) fat content is calculated as fold changes: TAG equivalent content/protein content of Stim upstream candidate gene-TtI On or Stim upstream candidate gene-TtI On/Off negative control 
normalized with respect to TAG equivalent content/protein content of day 0 Stim upstream candidate gene-Ttl Off flies. For details on the nomenclature of Ttl On, Ttl Off, Ttl On negative control, Ttl Off negative control see Figure 3.

A. Reduction of Galpha49B expression by Galpha49B-Ttl On for 48 hours promotes an increase of body fat storage at day 21 after the induction time point. B. Reduction of $G \gamma 1$ expression by $\mathrm{G} \gamma 1-\mathrm{Ttl}$ On for 48 hours promotes an increase of body fat storage at day 21 after the induction time point (Gr1 RNAi1). (C) Reduction of Plc21C expression by PIc21C- TtI On for 48 hours promotes an increase of body fat storage at day 0,11 and 21 after the induction time point (PIC21C RNAi1). For details of the underlying genetic constitution of the flies and experiments see 2.1.1, 2.1.2 and 2.1.4, 2.3.1, 2.3.3. Error bars represent the standard deviation of samples. ${ }^{*}=p<0.05,{ }^{* *} ;=p<0.01,{ }^{* * *}=p<0.001$ (Student's t-test).

First round data was kindly done by A. F. Borcherding (Borcherding, 2014).

Furthermore, Akh/AkhR signalling is required for lipid mobilization under fasting conditions (Gáliková et al., 2015; Grönke et al., 2007; Lee and Park, 2004). The results in the study presented here extend these previous studies by showing that transiently expressed Stim RNAi, and the reduction or lack of gene activities that act upstream of or in parallel to Stim cause a long-term fat storage increase. Thus, these results further support the central role of store-operated calcium entry (SOCE) in coordinating plasma membrane receptor stimuli in response to fasting, which are needed to control lipid mobilization. It further supports that calcium signalling plays a conserved role in fat storage control. Moreover, experimental loss-of-function of ORAI1 (the ORAl gene encodes a conserved component of SOCE) caused abnormal amounts of fat accumulated in heart and skeletal muscle of mice (Maus et al., 2017). Consistently, overexpression of ORAl gain-of-function transgene substantially reduced fat storage (Baumbach et al., 2014a), it will interesting to test whether reducing ORAI gene activity in the fat body tissue of flies promote their fat storage.

\subsubsection{LISO provides an attractive model for obesity and related diseases research}

Obesity has been defined as abnormal or excessive fat storage, which poses risks to human health (Garrow, 1988; WHO Consultation on Obesity, 2000). My study shows typical characteristics of obesity in Stim-Ttl flies that are common to human. First, at the organismal level, the fat content increase over time visibly affects the abdomen of the affected flies which are swollen due to expanded fat body tissues in day 21 StimTtl On flies, which represent the functional similar organ of the human white adipose tissue and liver (Padmanabha and Baker, 2014). Accordingly, after Stim-Ttl, the increased fat storage also causes substantial weight gain. Secondly, at the molecular 
and cellular level, the continuous increased fat storage, mainly in forms of TAGs, are stored as excessive lipid droplets (LDs) in larger fat body cells. These data are consistent with the findings on adipocyte enlargement and LD expansion in human obesity (Guilherme et al., 2010; Walther and Farese, 2012). LDs can also store sterol esters in both Drosophila and mammals (Pol et al., 2014; Walther and Farese, 2012). However, Drosophila is cholesterol-auxotroph (Niwa and Niwa, 2011). Thus, it will be interesting to see in future studies whether there is also a contribution of sterol esters to the increased fat storage in Stim-TI flies and, if so, how such an increase would be achieved.

In addition, excessive fat storage in the human abdomen, also called as android fat, has been linked to higher disease risks such as the metabolic syndrome (Smith, 2015; Walker et al., 2014), cardiovascular disease (Bays, 2014) and cancer (Britton et al., 2013). Similarly, LISO flies displayed impaired physiological effects such as lipid mobilization under starvation conditions, even though flies significantly survived longer. This observation is in line with other fly obesity models which are based on impaired lipid mobilization pathway genes such as loss-of-function mutation in the TAG lipase gene bmm (Grönke et al., 2005), and the loss-of-function AkhR mutation (Grönke et al., 2007). Interestingly, obese subjects who have been fasted 17 hours or exercised for 45 minutes displayed significantly lower plasma fatty acids (FAs) than lean subjects (Klein et al., 1965). However, one should be cautious when one relates plasma fatty acidy level to adipose tissue lipid mobilization (Klein et al., 1965). In fact, decreased catecholamine-stimulated lipolysis has been identified in human obese individuals, which is an important factor for the development and maintenance of obesity (Arner and Langin, 2014; Langin et al., 2005). Although the exact fatty acid release in starving LISO flies is unknown yet, the compromised Akh stimulated under fasting likely maintains the increased body fat storage, which might provide clues for the maintenance of LISO development.

It is interesting to note that LISO flies display normal functional glycogen mobilization during starvation. Similarly, glycogen mobilization is normal in loss-of-function $A k h R$ mutant flies but significantly impaired in Akh loss-of-function mutant flies under starvation condition (Gáliková et al., 2015). A recent study shows that knockdown of the receptor gene (CrzR) of corazonin, a homologue of Akh, impaired glycogen mobilization in starved flies (Kubrak et al., 2016). These findings indicate that glycogen utilization in the fasting flies is regulated by the Akh and Crz signaling. 
Another interesting similarity between fly and human obesity is the fact that obesity causes cardiac lipotoxity and heart dysfunction (Birse et al., 2010; Diop et al., 2015). Concerning the extreme obesity phenotype of LISO flies, it will be very interesting to check the potential lipotoxic cardiomyopathy in LISO flies. Eventually, consistent with extensive studies on the association between obesity and mortality risks (Pischon et al., 2008; World Health Organization, 2008), different candidate disease factors such as cardiomyopathy may contribute to a shorter median lifespan of LISO flies. It is noteworthy that obesity in flies also affects physical abilities as reflected in the impaired climbing performances of the affected individuals. Typically, impaired climbing ability is a putative hallmark of Parkinson (Feany and Bender, 2000), muscle dystrophy (Shcherbata et al., 2007), and Alzheimer diseases (Chakraborty et al., 2011). It has been identified that lipid droplet accumulation in glia results from neuronal mitochondrial dysfunction and ROS generation in both Drosophila and mice; the onset of neurodegeneration can be significantly alleviated by reducing Lipid droplets accumulation and lipid peroxidation in glia via targeted lipase overexpression in adult flies (Liu et al., 2015). Whether this impairment of obese flies is also due to neuronal defects as described in the mentioned diseases, or simply a consequence of the excessive fat storage of LISO flies, cannot be deciphered at moment. These parallels suggest that like in human obesity, LISO not only displays the typical abnormal fat storage, but also substantially augments detrimental risks to health. Therefore, LISO can serve as novel and attractive model for obesity and obesity related diseases.

\subsection{Dysfunctional energy homeostasis causes LISO}

Earlier studies have shown that both hypomophic mutations of the Drosophila inositol 1,4,5-trisphosphate receptor (InsP3R) gene Itp-r83A and the acute knockdown of Stim causes hyperphagia in adult male flies (Baumbach et al., 2014a; Subramanian et al., 2013). Consistently, I observed that Stim-Ttl was sufficient to directly trigger a persisting increased food intake of adult male flies at the beginning of Ttl, while increased food intake that derived from the $29^{\circ} \mathrm{C}$ temperature shift diminished when flies were transferred back to $18^{\circ} \mathrm{C}$. In addition, Stim-Tdl, which does not involve a temperature change, also resulted in hyperphagia of adult male flies in the following 7 days after Stim-Tdl. Since it is difficult to distinguish between primary increase of food intake provoked by Stim-Tdl (induced by RU486 containing liquid food) and augmented food intake due to ensuing ingestion of more RU486 (leading to elevated Stim-Tdl), the liquid food intake during Stim-Tdl was not checked. Instead, the intake 
of food was assessed with a capillary feeder assay from day 0 onwards after StimTdl. The time needed to adapt to capillary feeding might be the possible reason why Stim-Tdl flies had same food intake level as compared to flies where Stim RNAi was not expressed at day 1. Nevertheless, both Stim-Ttl and Tdl caused early fly hyperphagia during LISO development. Moreover, it appears that the food intake is the key determinant of increased fat storage of Stim-Tdl flies regardless of the different ratios the nutrients were provided. In fact, increased food intake is also regarded as the major driver of human obesity development (Swinburn et al., 2009; Vandevijvere et al., 2015). Accordingly, my lipid incorporation assay results show that increased ingested glucose of Stim-Tdl males was efficiently converted as revealed by the transfer of ${ }^{14} \mathrm{C}$ from labeled carbohydrates in the food to newly synthesized neutral lipids and phospholipids in the flies. In Stim Tdl On flies, this ${ }^{14} \mathrm{C}$ incorporation rate of neutral lipids was significantly increased. This finding is reminiscent of earlier studies where the obesity blocking neurons c673a-Gal4 targeted brain neurons of the flies were silenced, and their impairment caused increased food intake as well as a higher ${ }^{14} \mathrm{C}$ radioactivity signal in lipid fraction after feeding them with ${ }^{14} \mathrm{C}$ labeled food (Al-Anzi et al., 2009). Moreover, Stim-Tdl increased flies' food intake by 1.5 fold, while the Stim-Tdl increased flies' ${ }^{14} \mathrm{C}$ labeled food on neutral lipids by 5 times. Taken together, these results confirm a direct role of increased food intake in the fat accumulation of LISO flies, and further point to other factors also contributing to higher fat storage.

I also found that Stim-Ttl On flies could accumulate fat storage under pair-fed condition, i.e. offering a limited amount of food to the flies, even though the extent of fat storage increase is less as compared to flies living under ad libitum feeding conditions. This result confirms earlier results reported by (Baumbach, 2014). In addition to food intake, earlier studies also provide evidence that additional factors could influence the de novo fatty acid synthesis or lipolysis, which finally caused abnormal fat storage (Al-Anzi et al., 2009; Hehlert, 2016; Katewa et al., 2012). It will be interesting to learn how Stim-TI causes higher rates of ${ }^{14} \mathrm{C}$ incorporation, which further contribute to LISO development. Nonetheless, the findings reported here strongly support the argument that increased food intake drives the development of LISO, and may also indicate that reduced energy expenditure participates in abnormal fat storage.

Reduced energy expenditure in response to reduced physical activities has been correlated with a positive energy balance in obese humans (Church et al., 2011; 
Roda et al., 2016). In support of this correlation, I reported that, t-LISO flies displayed reduced locomotor activity during both day-time and night-time after Stim-Ttl. Moreover, d-LISO flies showed a significantly reduced metabolic rate as revealed by the $\mathrm{CO}_{2}$ production measurements on Stim-Tdl flies. Interestingly, a previous study did not reveal a difference in locomotor activity and metabolic rate after 6 days of Stim RNAi expression and the control flies (Baumbach et al., 2014a). One possible reason for this difference may lie in experimental conditions, i.e. the $29^{\circ} \mathrm{C}$ heat shock that was used in the previous Stim RNAi induction experiments (Baumbach et al., 2014a), as temperature is known to increase the locomotor activities (Lee and Montell, 2013) as well as the metabolic rates of flies (Berrigan and Partridge, 1997; Yatsenko et al., 2014). The induced locomotor activity at $29^{\circ} \mathrm{C}$, and the corresponding metabolic rate increase possibly undermine the reduction caused by LISO. My results described for LISO flies is therefore in line with recent study on flies lacking octopamine, which resulted in reduced physical activity, reduced metabolic resting rate, as well as increased body fat storage of the flies (Li et al., 2016). Realtime recordings document the reduction of locomotor activity occurred at day 3 after Stim-TtI. In mice, diet-induced obesity in turn causes dysfunction of basal ganglia (D2 dopamine receptor signalling), which hamper the physical activity (Friend et al., 2016). Therefore, the observed reduced locomotor activity and metabolic rate of LISO flies corroborate with decreased of energy expenditure of the flies, which likely aggravate the LISO phenotype via vicious circle manner.

\subsection{Molecular and cellular mechanisms underlying LISO}

\subsubsection{Stim-Transient Induced RNAi expression (Stim-TI) mediates long-term impairment of STIM function possibly via long-lasting small interfering RNA}

The Stim mRNA levels remained long-term reduced after Stim-TI. Similarly, AkhR-TtI entailed long-term lower AkhR mRNA levels in the following 10 days. Usually, the long-term impairment of Stim expression could be due to long-lasting siRNAs which mediate the RNAi effect (Elbashir et al., 2001; Zamore et al., 2000). In C. elegans, exogenously or endogenously derived siRNAs can exert trans generational gene silencing via recruitment of RNA-dependent RNA polymerases (RdRPs) to targeting mRNAs, which is in order to produce more endogenous siRNA (Billi et al., 2014; Maniar and Fire, 2011; Vastenhouw et al., 2006). In addition, a ribonucleoprotein complex, which has RdRP activity was reported in human cells (Maida et al., 2009). Whether the Drosophila genome encodes such a RdRP is still controversial (Lipardi 
and Paterson, 2009, 2010). My data, however, suggest that siRNA generated from transiently induced RNAi (Ttl) can play a role over a period of 10 days in providing the long-term impairment of Stim expression, as AkhR-Ttl flies displayed higher levels of siRNAs at day 10 after transient $A k h R$ RNAi expression, although some reduction of siRNAs was detectable. Moreover, compared to the body fat content increase of male flies subjected to simultaneous expression of Stim-RNAi and Stim cDNA through the respective transgenes (Stim RNAi\&RA-TI On) (Figure 19 (Preliminary data) A), the increase of fat body content of flies co-expressing StimRNAi and Stim-RNAi resistant cDNA through the respective transgenes was only transiently suppressed at day 0 (Figure 20 (Preliminary data) B), but not at later stages. Hence, co-expression of Stim RNAi resistant cDNA transgene cannot suppress the long-lasting body fat storage increase of Stim-Ttl. Therefore, it appears that long-lasting existence of siRNA generated from Stim-Ttl renders long-term impairment of STIM function through RNAi rather than the feedback loop regulation on the transcription of Stim. Last but not least, it is known that RNAi can participate in heterochromatin formation, thereby linking siRNAs to heterochromatin or epigenetic silencing of gene expression (Bühler and Moazed, 2007; Fagegaltier et al., 2009; Holoch and Moazed, 2015). Therefore, in addition to the mRNA degradation mediated by siRNA, the siRNA mediated heterochromatin or epigenetic silencing mechanism of siRNA action and its long-term effect cannot be excluded.

\subsubsection{Long-term impairment of STIM function is accompanied by alterations of gene expression in multiple pathways}

RNA-seq analysis of fat body tissue transcripts revealed STIM-dependent alterations of the gene expression pattern, including genes that encode cellular and inter-organ systemic signalling components as well as genes controlling cell growth and lipid metabolism.

Genes encoding neuropeptides, hormones, and receptor binding proteins were differently expressed in fat body tissues of LISO and control flies. For example, the insulin secretion promoting gene CCHa2 (Sano et al., 2015) was substantially upregulated, while the insulin secretion suppression gene Lst (Alfa et al., 2015) and the insulin peptide binding protein gene ImpL2, which negatively insulin peptide's function (Honegger et al., 2008), were down-regulated. These results point to increased insulin production and secretion, which is consistent with both, the augmented food intake and energy storage in LISO flies. In addition, the Akh 
receptor gene AkhR (Bharucha et al., 2008; Grönke et al., 2007; Kim and Rulifson, 2004) was also altered, as is the Akh secretion-related neuropeptide gene Tk (Nässel et al., 1995; 1999). These findings in RNA-seq analysis may link the possible dysfunction of Akh signalling to the impaired lipid mobilization found in LISO flies under starving conditions.

Regarding the downstream cellular pathway of insulin and Akh signalling, upregulation of the adenylate cyclase (AC) genes, and down-regulation of genes encoding calcium ion binding proteins e.g. mitochondrial substrate/solute carrier protein aralar1 (Del Arco et al., 2000) were identified with RNAseq analysis. These findings are in line with what has been observed with SOCE-deficient mouse cells. Such cells displayed a reduced cytosolic cyclic adenosine monophosphate (cAMP) level and the cAMP-dependent phosphorylation of the hormone-sensitive lipase (HSL) was decreased (Maus et al., 2017). cAMP is produced by plasma-membraneassociated AC, the activity of which is regulated by Gs protein-coupled receptor signalling (Hepler and Gilman, 1992; Ritter and Hall, 2009) as well as by $\mathrm{Ca}^{2+}$ levels and by ORAI (Maus et al., 2017; Willoughby and Cooper, 2006; Willoughby et al., 2012). Therefore, it is tempting to hypothesize that long-term impairment of STIM function inhibits the store-operated calcium entry, which in turn hampers the function of calcium ion binding proteins and AC activities. In the long-term, up-regulation of $\mathrm{AC}$ genes might be a complex compensatory consequence rather than the cause of LISO development. Further studies are required to test this hypothesis in Drosophila by addressing the fat body cell cytosolic CAMP level when STIM function is impaired. Regarding calcium ion binding proteins, it would be interesting to check its functional link to fat storage in future studies.

I also observed the up-regulation of genes, which encode microtubule, cilia and tubulin binding proteins. This finding is consistent with the increased fat body cell size of LISO flies. In fact, cilia dysfunction has already been linked to obesity in mammals (Oh et al., 2015). In addition, lipid droplets (LDs) need to be transported by the microtubule system with the help of motor proteins in Drosophila embryos (Arora et al., 2016; Welte, 2015), and the formation of large LDs involves microtubuledependent fusion of LDs (Ariotti et al., 2012). It is also reported that cytoskeletal remodeling is essential for LDs maintenance during lipolysis (Orlicky et al., 2013). These earlier observations are consistent with my finding that the genes encoding components of microtubule proteins are differentially regulated in fat body cells, in which SOCE is impaired. Future studies are needed to investigate how cilia may 
participate in adipocyte function, and how the dynamics of LDs are controlled by microtubule system in the fat body cells of adult flies. Furthermore, it is important to note that my current RNA-seq data cannot predict whether these genes are directly regulated by SOCE or the consequence of the increased food intake. It will therefore be important to learn which of the genes with altered expression patterns are direct target genes of calcium-regulated transcription factors with up-stream regulator analysis and further functional studies.

Finally, the transcripts of a large number of genes involved in lipid metabolism and fatty acid $\beta$-oxidation were enriched. Their products are associated with metabolismrelated organelles such as LDs, mitochondria and peroxisomes. This finding is consistent with the abnormal fat storage in LISO flies. Moreover, it has been shown that mitochondrial gene expression, fatty acid oxidation as well as the mitochondrial function in electron transport chain were reduced or impaired in SOCE-deficient mammalian fibroblasts (Maus et al., 2017). Future studies may uncover whether impaired mitochondrial functions affecting the mitochondrial membrane potential (MMP) and NADH levels and damaged mitochondria can be also found in Drosophila fat body cells of LISO flies.

\subsubsection{Akh signalling contributes to LISO by promoting the food intake}

It is of interest, that Akh signalling is required for the increased food intake during LISO development. There is evidence from earlier studies that Akh signalling also affect brain functions. In fact, $A k h R$-Gal4 drives gene expression not only in fat body tissues, but also in gustatory neurons of the adult subesophageal ganglion (Bharucha et al., 2008). Although in situ hybridization and antibody staining failed to identify $A k h R$ expression in gustatory neurons, $A k h R$ mRNA was independently found in Drosophila gustatory system by genome-wide microarray screen (reported in Bharucha et al., 2008). Moreover, it has been demonstrated that Akh directly modulates the activities of four interoceptive SEZ neurons (ISNs) located in the subesophageal zone (SEZ). Activity of these neurons promote sucrose consumption, while ISNs activity did not increase in the $A k h R$ mutant (Jourjine et al., 2016). Ectopic over-expression of Akh in fat body tissue increases food intake, whereas food intake is reduced in Akh loss-of-function mutants (Gáliková et al., 2017). Furthermore, low nutrient condition can trigger the secretion of Akh from the corpora cardiaca (Braco et al., 2012; Kim and Rulifson, 2004; Lee and Park, 2004). Therefore, it seems that 
increased Akh signalling not only depletes the fat body nutrient storage, but also is also sufficient to enhance the food intake of flies.

My results suggest that Stim-Ttl causes Akh resistance in the fat body cells of LISO flies and that Stim acts downstream of Akh/AkhR signalling. Therefore, it appears reasonable to assume that after Stim-Ttl, the impairment of STIM function prevents the lipid mobilization in response to Akh signalling, which in turn causes the continuous long-term increase of body fat storage. Furthermore, the up-regulation of AkhR mRNA was observed, but no significant change of the tobi mRNA level was detected. It is known that that tobi is a common downstream target gene of insulin and AKH signalling (Buch et al., 2008). Therefore, the increase of AkhR mRNA level might be a compensatory effect, as Akh signalling is impaired due to long-term functional impairment of STIM in LISO fly fat body tissue. It is reasonable to propose that an impairment of STIM function in fat body tissue promotes the food intake via increased $\mathrm{AKH}$ secretion. This proposal is based on the finding that RNA-seq analysis revealed an up-regulation of the Akh secretion related neuropeptide gene Tachykinin (Tk). Tk protein induces Akh secretion of locust corpora cardiaca in vitro (Nässel et al., 1995, 1999). Assuming that the Tk protein has the same function in flies, these findings would indicate a post-transcription regulation of Akh signalling during LISO development. In fact, I observed no difference in the Akh mRNA expression level by RT-qPCR when comparing the transcript levels of LISO and untreated control flies, but there was increased Akh secretion from the corpora cardiaca of Stim-Ttl as revealed by Akh immunostaining analysis. The increased Akh secretion was positively correlated with the increased food intake of the LISO flies. Moreover, the higher food intake after Stim-Tdl was abolished in Akh loss-of-function mutant flies. As a consequence, unlike Akh heterozygous flies, the homozygous $A k h$ loss-of-function flies failed to accumulate fat over a period of 15 days after Stim-Tdl. My data therefore support a model where long-term impairment of STIM function by Stim-TI results in dysfunctional Akh signalling on fat body tissue, which in turn stimulates Akh secretion via Tachykinin. The resulting Akh level in hemolymph further promotes food intake.

Akh signalling is thought to be functionally homologous to mammalian glucagon (Bharucha et al., 2008; Kim and Rulifson, 2004) as well as $\beta$-adrenergic signalling (for review, see (Gäde and Auerswald, 2003; Van der Horst et al., 2001; Schlegel and Stainier, 2007)). It is also known that glucagon and glucagon-like peptide (GLP1 ) inhibit the food intake (reviewed in (Woods et al., 2006)). In this context, $\beta_{1}$ and $\beta_{2^{-}}$ 
adrenergic receptors were first shown to inhibit food intake based on $\beta$-adrenergic blockers and agonists studies in rats (Borsini et al., 1982; Hajinezhad and Shohreh, 2016; Racotta and Soto-Mora, 1993). Moreover, it was shown that the $\beta_{3}$-adrenergic receptor is involved in the regulation of food intake reduction (Kanzler et al., 2011). This apparent difference between Akh's role in promoting food intake and $\beta$ adrenergic signaling's role in inhibiting food intake points to an alternative or additional functional homologue signalling in the food intake control of mammals as compared to Akh in Drosophila. A possible functional homologue candidate is Ghrelin (Petsakou and Perrimon, 2016), which has been shown to participate in the regulation of the food intake (Nakazato et al., 2001; Wren et al., 2000, 2001).

It is noteworthy to mention that although Akh signalling has been intensively studied in Drosophila, it remains unknown how fat body-secreted factors regulate Akh secretion from corpora cardiaca. My results raise the possibility that fat body tissues could secrete the Tachykinin peptide to increase corpora cardiaca secretion of Akh. In mammals, functional counterparts of Tachykinin are substance $P(S P)$, neurokinins $A$ and $B$, which have been shown to provoke the release of both insulin and glucagon from the pancreas (Schmidt et al., 2000). Further studies are required to test this hypothesis in Drosophila and to examine whether the secretion of Tachykinin is increased from fat body tissue and whether its receptor is expressed in the corpora cardiaca during LISO.

\subsubsection{Long-term impairment of STIM function in fat storage tissue contribute to selective insulin resistance and lipid synthesis in LISO flies}

As early as in 1889, the German scientists Minkowski and von Mering hypothesized that a substance secreted from pancreas was linked to metabolic control, which was finally identified as the insulin peptide in 1921 by the Canadian scientists Banting and McLeod (Wilcox, 2005). More than hundred years of studies have led to an understanding of the regulation of insulin secretion and insulin signalling. Moreover, the insulin signalling pathway is found to be well-conserved from Drosophila to human (Alfa and Kim, 2016). In the wake of worldwide epidemics of obesity and type-2 diabetes, studies in mammals have demonstrated that obesity causes systemic insulin resistance via the cytokine Tumor Necrosis Factor- $\alpha$ (TNF- $\alpha$ ) acting in adipose tissue as well as other cytokines and lipid mediators (Gregor and Hotamisligil, 2011; Hotamisligil et al., 1993, 1995, 1996; Savage et al., 2007; Wellen and Hotamisligil, 2003; Wilcox, 2005). Insulin resistance is normally defined as an 
impaired biological response, classically referring to reduced sensitivity to insulinmediated glucose uptake, the metabolism or storage in adipocytes and in skeletal muscle (Kahn and Flier, 2000; Wilcox, 2005). Insulin resistance was also proposed as a complex inter-connected process that involves insulin, glucose, and the lipid metabolism (McGarry, 1992). Consistent with the view, hepatic de novo lipogenesis has been linked to insulin resistance (Ameer et al., 2014). Therefore, the concept of selective insulin resistance has been postulated: insulin receptor singling's role in promoting the glucose uptake and its storage and repressing glucogenesis, while de novo lipogenesis via the lipid synthesis transcription factor SREBP1c or the regulator ChREBP, is maintained (Sanders and Griffin, 2016). In terms of insulin signalling regulation, mammalian adipose tissue was recognized as a key endocrine organ, which interacts with immune cells, brain, liver and pancreas to form an insulin signalling regulatory network by sensing metabolism signals and by secreting important hormones such as leptin, and TNF (Gregor and Hotamisligil, 2011; Hussain et al., 2016; Kahn and Flier, 2000; Kershaw and Filier, 2004; Zhang and Zhang, 2010). Similarly, with respect to insulin signalling, the components of interorgan communication networks between the Drosophila fat body tissue, immune cells, insulin producing cells and the brain are remarkably conserved (Droujinine and Perrimon, 2016; Hotamisligil, 2017). Although over the last decades, we have gained a good understanding of how obesity contributes to insulin resistance, the question whether insulin resistance exacerbate the development and maintenance of obesity remained elusive. LISO offers a possible Drosophila model to address this question by showing how selective insulin resistance is developing in response to long-term impairment of STIM function in fat body tissue, and as a consequence, how it contributes to the development of obesity. Several lines of evidence support this idea. First, the expression of insulin secretion promotion gene CCHa2 (Sano et al., 2015) was significantly up-regulated directly after Stim-TI, and maintained at measurable levels during the following 10 days. Secondly, expression of the insulin peptide inhibitor gene ImpL2 (Honegger et al., 2008) and the insulin secretion suppressing gene Lst (Alfa et al., 2015) was substantially decreased in fat body tissue at day 10 after Stim-TI. These results suggest an increased secretion of insulin signalling promoting hormone from fat body. Thirdly, dILP-2 secretion from InsulinProducing Cells (IPCs) was increased after Stim-TI. These data show that long-term impairment of STIM function in fat body tissue remotely promotes insulin secretion and facilitates insulin binding with peripheral insulin-dependent tissues. It is important to note that the correlation between the expression of the fat body hormone genes and their secretion should be established. More importantly, the increase of 
hemolymph dILP-2 level after Stim-TI needs to be identified. It is also noteworthy to mention that increased insulin secretion after Stim-TI complements the finding that Stim-TI causes an increased food intake, and that insulin secretion has been associated with the feeding control in both Drosophila (Barry and Thummel, 2016; Park et al., 2014a) and mammals (Wilcox, 2005).

Increased insulin secretion was shown to stimulate the peripheral tissue insulin signalling cascade of which the phosphorylation of Akt is one of key events in both mammals (Boucher et al., 2014; Wilcox, 2005) and Drosophila (Scanga et al., 2000). Since insulin secretion is increased in LISO flies, one can expect that the Akt phosphorylation level will be up-regulated in fly peripheral tissues. Surprisingly, 4 days after Stim-Tdl, the peripheral tissues of the flies showed a reduced Akt phosphorylation level at the Ser505 residue, which causes Akt to be active (Scanga et al., 2000). Furthermore, there was no significant difference of Akt phosphorylation level at Thr342 residue which also activates Akt (Schleich et al., 2014). Moreover, the fat body cells of Stim-Ttl flies did not show increased new DNA synthesis based on nucleus as revealed by the EdU incorporation assay (Fernández-Hernández et al., 2013). This observation indicates that the fat body cells do not undergo active proliferation. In line with this finding, Drosophila intestinal-stem cells did also not show increased proliferation rate when functions of calcium signalling genes (including Stim) or insulin receptor gene $(\operatorname{InR})$ were impaired (Deng et al., 2015). Moreover, the fat body tissue of Stim-Ttl flies showed an increased expression of insulin signalling target gene $4 E B P .4 E B P$ is a direct target gene of the conserved transcription factor Forkhead box, sub-group O (FOXO), which is repressed through phosphorylation by the active Akt in response to active peripheral insulin signalling (Jünger et al., 2003; Puig et al., 2003). Also, expression of the Glucokinase (GCK) homolog Hexokinase-C (glycolytic), which is encoded by Hex-C, was reduced in the fat body of LISO flies. Similar to the function of its homolog in mammalian liver, Hex$C$ is required in the fat body tissue to maintain the normal circulating glucose level (Barry and Thummel, 2016; Haeusler et al., 2015; Massa et al., 2011; Velho et al., 1996). Reduction of Hex-C expression in fat body tissues points to the functional impairment of fat body tissue in glucose clearance. Taken together, these data strongly indicate that the fat body tissue of LISO flies becomes insulin-resistant after Stim-TI. This conclusion is consistent with the finding that LISO flies showed normal glycogen storage level, indicating that glucose intake and storage was not increased under hyperphagia and elevated insulin secretion condition. However, six days after Stim RNAi overexpression, there was no detectable effect on the sugar levels, i.e. 
glucose and trehalose, in the hemolymph of the flies (Baumbach et al., 2014a). Since temperature-associated stress substantially affect glycogen homeostasis (Klepsatel et al., 2016), it would be valuable to check if circulating sugar levels of flies where Stim-TI was drug-induced instead of by the temperature shift. Moreover, a oral glucose tolerance test (starvation, and refed with glucose) (Barry and Thummel, 2016) could be used to determine whether LISO flies have impaired circulating glucose clearance under feeding condition.

The observed accumulation of body fat storage suggests that lipid biosynthesis and storage was not impaired in LISO flies. In fact, I observed no down-regulation of expression of genes which encode factors involved in lipid biosynthesis at day 10 LISO flies, which include fatty acid synthase gene $d F A S$, the acetyl coenzyme $A$ synthase gene $d A C S$, the $\mathrm{Mg}^{2+}$-dependent PA phosphatase gene dLipin, the acylCoA:diacylglycerol acyltransferase (DGAT) gene $m d y$ and the lipid synthesis transcription factor sugarbabe (sug) (Mattila et al., 2015). However, daw, the gene that encodes the transformation growth factor $\beta$ family Activin-like ligand Dawdle (daw) that promotes lipid biosynthesis (Mattila et al., 2015), was transcriptionally upregulated. These results indicate that the transcription of de novo lipid biosynthesis genes is likely to be normal in Stim-Ttl flies and possibly controlled at the posttranscriptional level. This notion is further sustained by the genetic interaction analysis, since Stim-Tdl flies showed an increase of body fat storage despite the insulin signalling components were impaired (loss of dllp2-3,5, and dllp6 (Figure 20 (Preliminary data) A). Further studies are required to identify the regulator of lipid biosynthesis under selective insulin resistance condition using the Stim-TI flies.

Studies in mammals have pointed out, that hepatic de novo lipogenesis and fatty acid re-esterification are promoted under insulin resistance conditions, which drive up the ectopic excessive lipid accumulation in the liver (Sanders and Griffin, 2016; Savage et al., 2007). Since the fat body represents the organ that corresponds to the mammalian liver, insulin resistance in the fat body tissue of Stim-TI flies might aggravate the fat storage similar to hepatic de novo lipogenesis. In mammals, SREBP1c and ChREBP are the key transcriptional regulators of de novo lipogenesis (Sanders and Griffin, 2016), and the activity of the target of rapamycin complex 2 (mTORC2) might regulate glucose uptake in an Akt-independent manner, which drives the ChREBP-dependent de novo lipogenesis (Tang et al., 2016). In Stim-Ttl flies, I have not observed an increase of the expression of the $\mathrm{d} F A S, d A C S$ and $d A C C$, which are direct target genes of SREBP1 and ChREBP (Zhao et al., 2012). 
This indicates that the observed augmentation of body fat storage in Stim-TI flies is not dependent on a transcriptional enhancement of lipid biosynthesis genes or of their regulators. Aside from regulation of lipid biosynthesis genes expression, the key regulatory step of de novo lipogenesis of mammals is the synthesis of malonyl-CoA from an acetyl-CoA precursor which is catalyed by acetyl-CoA carboxylase (ACC), a process which is regulated by allosteric mechanisms (Sanders and Griffin, 2016). In Drosophila, dACC can be phosphorylated by AMPK, resulting in inactivation of dACC activity (Bland et al., 2010; Pan and Hardie, 2002). This regulation can be achieved in response to adrenaline or glucagon, and is mediated by Protein Kinase A. Based on this scenario, it would also be interesting to investigate whether dACC was activated via de-phosphorylation under Akh signalling resistance conditions. Last but not least, since the gene mdy was up-regulated shortly after Stim-TI (Baumbach et al., 2014b), it remains unclear how TAG synthesis and lipid droplet formation were post-transcriptionally regulated in the LISO flies, as dLipin's activity is possibly posttranscriptionally suppressed by the ER protein dTorsin in Drosophila fat body tissues (Grillet et al., 2016).

Taken together, my results so far suggest that fat body-specific Stim-TI promotes insulin secretion via the insulin secretion-promoting hormone $\mathrm{CCHa}$, by reducing the level of the insulin secretion-suppressing hormone Limostatin, and by reducing the amount of the insulin peptide binding protein ImpL2. It also causes selective insulin resistance and, most obviously, causes an increase of the fat storage via insulin signalling-independent lipid biosynthesis.

\subsubsection{Stim-Ttl reduces the expression of genes involved in lipolysis and mitochondrial functions}

Notably, RNA-seq and gene ontology analysis indicate that the Stim-TI in the early stage cause the later reduction of the expression of genes involved in lipolysis, mitochondrial functions and calcium responsive regulators. Moreover, RT-qPCR analysis confirms that the expression of the key lipase gene $b m m$ is significantly reduced in fat body tissue at day 10 after $34 \mathrm{~h}$ of Stim-Ttl. Bmm encodes the fly homolog of ATGL, the expression of which was reduced after a 6 days of Stim RNAi expression as reported in an earlier study (Baumbach et al., 2014b). Consistent with increased food intake in LISO flies, it has been found that Akt activates salt-inducible kinase3 (SIK3), which further decrease the expression of bmm during feeding (Wang et al., 2011). Concerning the insulin resistance condition, it remains to be 
investigated if other signalling can also regulate the activity of SIK3. On the other hand, Bmm was found to be a target gene of dFOXO and up regulated in fasted flies (Wang et al., 2011). As LISO develops under well-fed condition, the transcription regulation of $\mathrm{bmm}$ is likely not due to dFOXO. In fact, LISO still occurs in dFOXO loss-of-function flies. In addition to $\mathrm{bmm}$, the transcription of the mitochondrial fatty acid transporter gene CPT1, which encodes the enzyme carnitine Opalmitoyltransferase, and $d A C A D V L$, which encodes the enzyme acyl-CoA dehydrogenase involved in the mitochondrial $\beta$-oxidation process, were also significantly down-regulated at day 10 after Stim-TI. Consistent with these results, a recent study in mammals showed that the expression of ATGL, CPT1b, and ACADVL was also impaired in SOCE-deficient cells (Maus et al., 2017). Moreover, expression of the gene, which codes for the $\beta$ subunit of ATP synthase (ATP5B), ATPsyn $\beta$, was impaired at day 10, whereas the expression of the gene which encodes the alpha unit of ATP synthase (ATP5A) subunit was not affected as has been observed in SOCE-deficient mammalian cells (Maus et al., 2017). However, my RNA-seq analysis did not reveal a significant down-regulation of the electron transport chain (ETC) complex subunit homolog genes NDUFA1 (complex I), UQCRC1 (complex IV), COX4I1 (complex IV), which were shown to be repressed in mammalian SOCE-deficient cells (Maus et al., 2017). Mammalian cells displayed reduced uncoupling protein 2 (UCP2), a proton transporter in the inner mitochondrial membrane, which lacks a direct homolog in Drosophila. However, the NADH dehydrogenase (ubiquinone) $75 \mathrm{kDa}$ subunit (NDUSF1, ETC complex I) gene ND75 was down-regulated. Also, ATP synthesis genes were down-regulated, but in contrast to $\mathrm{bmm}$ and genes encoding factors involved in fatty acid $\beta$-oxidation, this reduction occurred later during LISO development. These observations provide additional support for the hypothesis that SOCE may control lipolysis mainly at the post-transcriptional level. In addition, it may also act on the lipolytic gene activity, and thereby activating fatty acid $\beta$-oxidation and the ATP production. Therefore, it would be interesting to check if ATP level in Stim-TI fat body tissue changes.

In addition to an impairment of lipolysis and fatty acid $\beta$-oxidation, the shuttling of NADH from cytoplasm to mitochondria also contributes to the ATP generation in ETC. Interestingly, the RNA-seq analysis indicates that all six malate-aspartate shuttle genes were substantially reduced, and the expression reduction of five of them were validated, i.e. arlar1, Mdh1, Mdh2, Got1 and Got2 by RT-qPCR at day 10 after Stim-Ttl. Besides, Stim-TtI resulted in a reduced mRNA level of Mdh1 as early 
as day 1 after the Stim-Ttl induction. This observation suggests that the impairment of the NADH transport from cytoplasm to mitochondria is an early consequence of Stim-Ttl, which impairs cytoplasmic NADH-based ATP production and decreases the cytoplasmic $\mathrm{NAD}^{+} / \mathrm{NADH}$ ratio. In mammalian cells, however, it seems like SOCEdeficient cells have their normal ability in replenishing $\mathrm{NADH}$ pool after its depletion (Maus et al., 2017). However, increased levels of NADH were found in SOCEdeficient cells under high-glucose medium condition (Maus et al., 2017). This is likely due to the ability of TCA cycle to supply NADH or FADH2 within the mitochondrial matrix, which appears to be normal in SOCE-deficient cells (Maus et al., 2017). In STIM-impaired fat body cells, a reduced $\mathrm{NAD}^{+} / \mathrm{NADH}$ ratio might promote fat accumulation through inhibiting $\mathrm{NAD}^{+}$-dependent deacetylases such as the sirtuin 1 (SIRT1) homolog (Banerjee et al., 2012; Canto and Auwerx, 2012; Reis et al., 2010). Loss of Sirt1 activity promotes fat accumulation (Banerjee et al., 2012), and results in the age-progressive development of obesity as well as in insulin resistance by reducing the Drosophila nuclear receptor dHNF4 levels (Palu and Thummel, 2016). In mammals, SIRT1 can modulate the activities of the transcription factor FOXO and PGC-1alpha by regulating their target gene expression (Brunet et al., 2004; Rodgers et al., 2005). Thus, functional activity of Sirt1 can be checked in future studies to see if it was involved in the development of LISO flies.

Moreover, dysfunctional regulation of Mdh1, one of malate-aspartate shuttle genes, has been linked to obesity in humans (English and Butte, 2007; Moreno-Viedma et al., 2016). In addition, aralar1 encodes a protein which is analogous to citrin, a protein isoform of Aralar encoded by SLC25A13 in human (Del Arco et al., 2000). Citrin-deficiency due to mutations of SLC25A13 causes neonatal intrahepatic cholestasis (NICCD) and adult-onset type II citrullinemia (CTLN2). One of the symptom provoked by NICCD is the fatty liver (Saheki and Kobayashi, 2002). Since Drosophila fat body tissue is also functionally analogous to the liver, these findings support the proposal that STIM affects fat storage through adjusting Mdh1 or Arlar1 mitochondria transporter activities. Moreover, a loss-of-function Mdh1 mutant was described (Eanes et al., 2009), may offer a material to study the direct causal link between Mdh1 and the body fat storage need to be investigated. In addition to the recent study in mammals (Maus et al., 2017), a more detailed analysis of the role of Mdh1 offers the opportunity to gain new insight into how SOCE may regulate fat storage. 
Interestingly, the mammalian genes ATGL, CPT1b, ACADVL are targets of the transcription factors PGC-1 $\alpha$ and PPAR $\alpha$, respectively (Maryam et al., 2010; Nikolić et al., 2012). In addition, Mdh1 is significantly down-regulated in muscle-specific PGC-1 $\alpha$ knock-out mice (Handschin et al., 2007). The expression of $P G C-1 \alpha$ and $P P A R \alpha$ are directly regulated by SOCE in adenylyl cyclase/cAMP/PKA-dependent manner (Maus et al., 2017). Consistent with these findings, I found that transcripts of the genes bmm (dATGL), CPT1, dACADVL, Mdh1 and spargel, encoding the PGC$1 \alpha$ homolog PGC-1 (Tiefenbock et al., 2010), were also down-regulated in Drosophila fat body tissue of Stim-Ttl flies. A mutation of the PGC-1 mimicked a high-fat diet-induced lipid accumulation and cardiac dysfunction induced by high-fat diet feeding in adult Drosophila (Diop et al., 2015). Also, overexpression of a dominant-negative form of CrebB in adult Drosophila fat body tissue promotes body fat storage (Baumbach, 2014; lijima et al., 2009). I have shown that Stim-TI caused long-term impairment of STIM function, which likely causes persistently reduced cytosolic $\mathrm{Ca}^{2+}$ level through preventing store-operated calcium entry (SOCE). Therefore, I propose that the mechanism of SOCE concerning the cell autonomous fat storage regulation is conserved from Drosophila to mammals. SOCE likely promotes lipolysis via adenylyl cyclase/cAMP/PKA mediated lipase phosphorylation and through transcriptional programming of lipid metabolism genes via the adenylyl cyclase/cAMP/PKA/CrebB/ PGC-1 pathway in Drosophila fat body tissues. It is important to note that the direct evidence for transcriptional activities of CrebB or PGC-1 need to be identified in LISO fly fat body tissue.

Mdh1 is directly or at least early regulated by $\mathrm{Ca}^{2+} /$ calcineurin-mediated transcription. In line with this, the calcineurin gene CanA-14F was later found significantly downregulated in Stim-TI fat body tissue. It remains elusive whether impaired expression of Mdh1 in Stim-TI fat body tissue is dependent on the calcineurin-dependent pathway. It is therefore of interest to analyze whether calcineurin may affect fat storage via $\mathrm{dPGC}-1$ regulated lipid metabolism.

\subsection{Fat body tissue is a key player in the inter-organ regulatory network to maintain energy homeostasis}

My work shows that Stim-TI not only interferes with cellular lipid metabolism of fat body tissue, but also affects the role executed by fat body tissue in the inter-organ regulatory network. It is the dysfunction of this organ regulatory network, which 
ultimately triggers the collapse of energy homeostasis in LISO flies. Stim-TI in fat body tissue affects the response of the fat body tissue to Akh and insulin signalling.

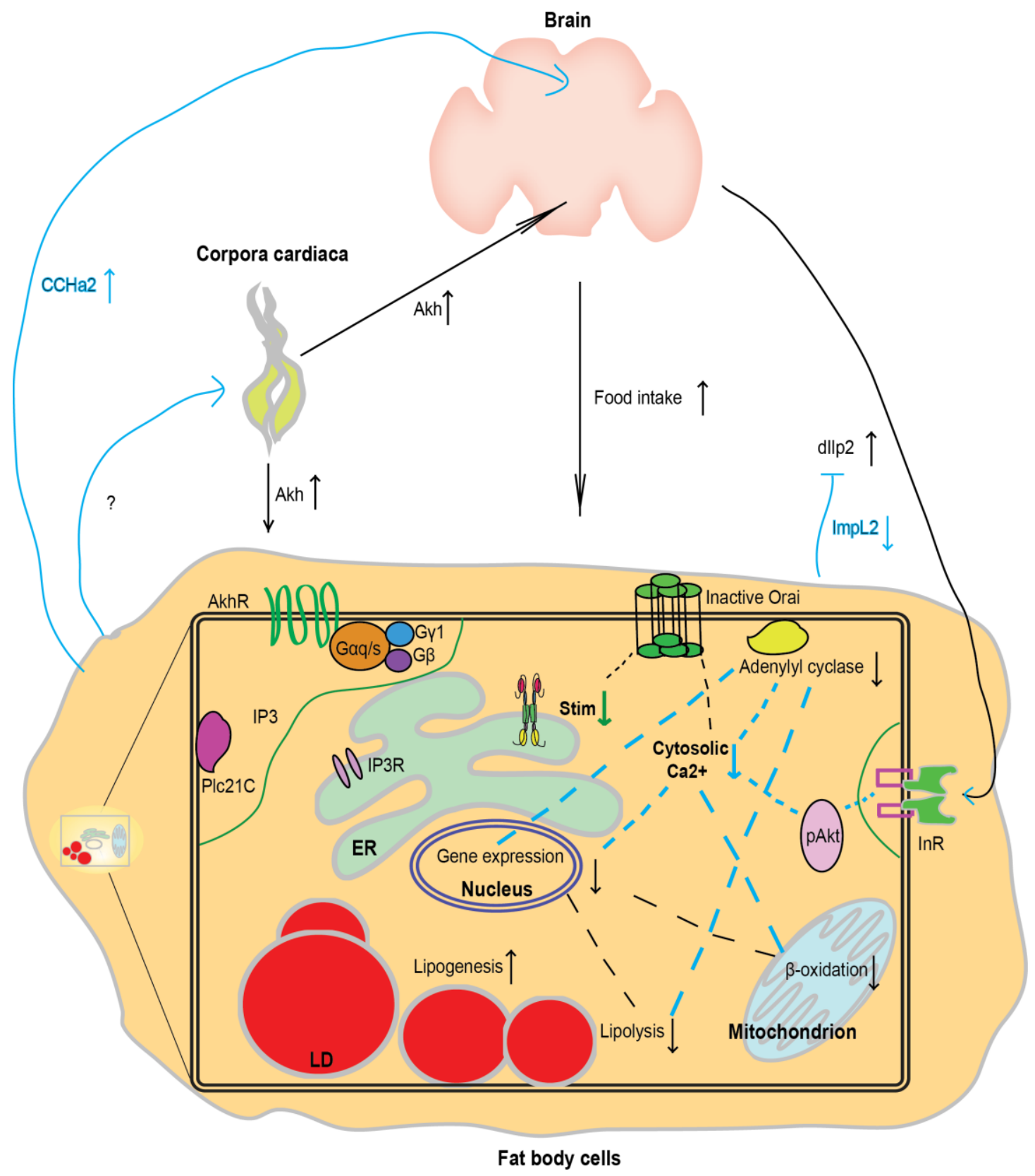

Figure 22. Scheme representing a model of how energy regulatory network is disturbed in the fat body cell and at inter-organ level in LISO flies

Stim-TI in fat body tissues entails the long-term functional impairment of STIM located on Endoplasmic Reticulumn (ER), which is very likely to reduce the cytoplasmic $\mathrm{Ca}^{2+}$ levels of fat body cells. Long-term functional impairment of STIM function undermines the store-operated calcium entry (SOCE) in response to AkhR/ Gaq, Gy1/Plc21C signaling pathway. Besides, the reduction of cytosolic $\mathrm{Ca}^{2+}$ level possibly inhibits the activity of adenylyl cyclase (AC), which is in response to AkhR/Gas signalling pathway. Moreover, the reduction of cytosolic $\mathrm{Ca}^{2+}$ level impairs the activation of Akt (the key component of insulin signaling pathway). In general, the long-term functional impairment of STIM causes Akh and insulin resistance (indicated by green line encircling the AkhR and InR receptor, respectively) in fat body cells of LISO flies. Further, at cellular level, reducing cytosolic $\mathrm{Ca}^{2+}$ level possibly directly impairs the 
function of mitochondria, and inhibits lipolysis via inhibiting AC's activity. In the long-term, reducing $\mathrm{Ca}^{2+}$ level reduces the expression of genes involved in lipolysis and $\beta$-oxidation. At inter-organ level, fat body cells possibly up-regulate hemolymph insulin peptide level (dILP2) via secreting more hormone peptide $\mathrm{CCHa} 2$ to promote the secretion of insulin-like peptide dILP2, and secreting less dILP2 binding protein ImpL2. In parallel, fat body cells promote the secretion of Akh from corpora cardiaca possibly via secreting Tk. Although increasing Akh level in the hemolymph does not promote energy mobilization due to AKH resistance, more secreted Akh peptides induce hyperphagia via brain. In summary, interfering STIM, the key node of energy regulatory networks, causes dysfunctions of energy homeostasis at cellular and inter-organ level. The figure is done with Adobe llustrator and Pathvisio (https://www.pathvisio.org/) based on figures in (Droujinine and Perrimon, 2016).

Moreover, long-term functional impairment of STIM function in the fat body tissue possibly promotes Akh secretion from the corpora cardiaca and insulin secretion from insulin producing cells via fat body derived hormones. Further, increased Akh increases the food intake via the brain controlled feeding behavior. After local interventions in fat storage tissue by Stim-TI, the collective dynamics in the interorgan network of energy homeostasis may be pushed to a new obesity developing state, which is likely caused by rewiring of the biological network (Hu et al., 2016). Such a model (Figure 22) is supported by the network theory study that a local interference with individual sub-network might change network-wide communication in a remote manner (Kirst et al., 2016). Moreover, network physiology studies also show that the failure of one organ system can rearrange physiological interactions of the entire network (reviewed in (Ivanov and Bartsch, 2014; Ivanov et al., 2016).

Regarding the general mechanism of obesity development in humans, different models have been proposed, e.g. 'set point', or 'settling point' theory; however, none of them can completely explain the wide spread of obesity and its related diseases (reviewed in Speakman et al., 2011). Although adult fly organ systems are simple comparing to human, the conserved regulation strategies, which are and will be identified in Drosophila, can inspire us to better understand the energy regulatory networks underlying human obesity and its related diseases. 


\section{Supplementary data}

A

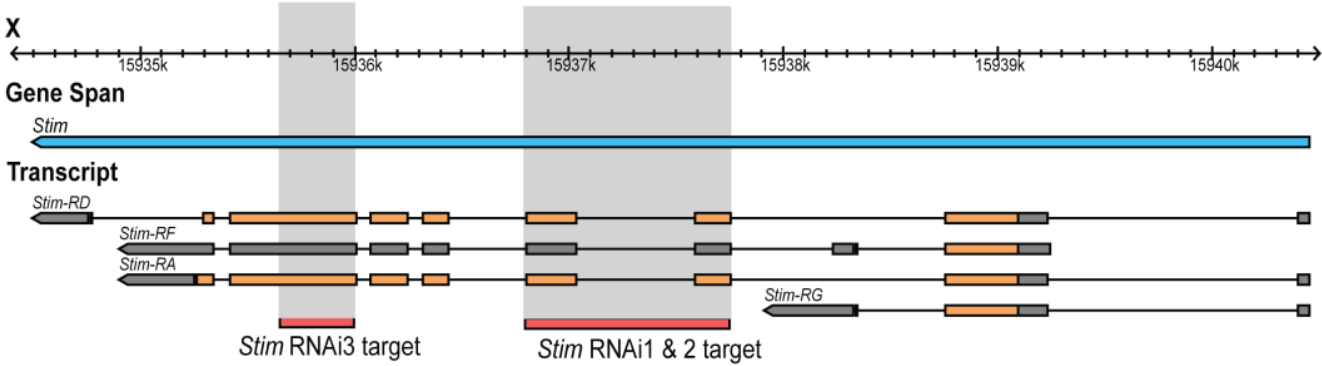

B
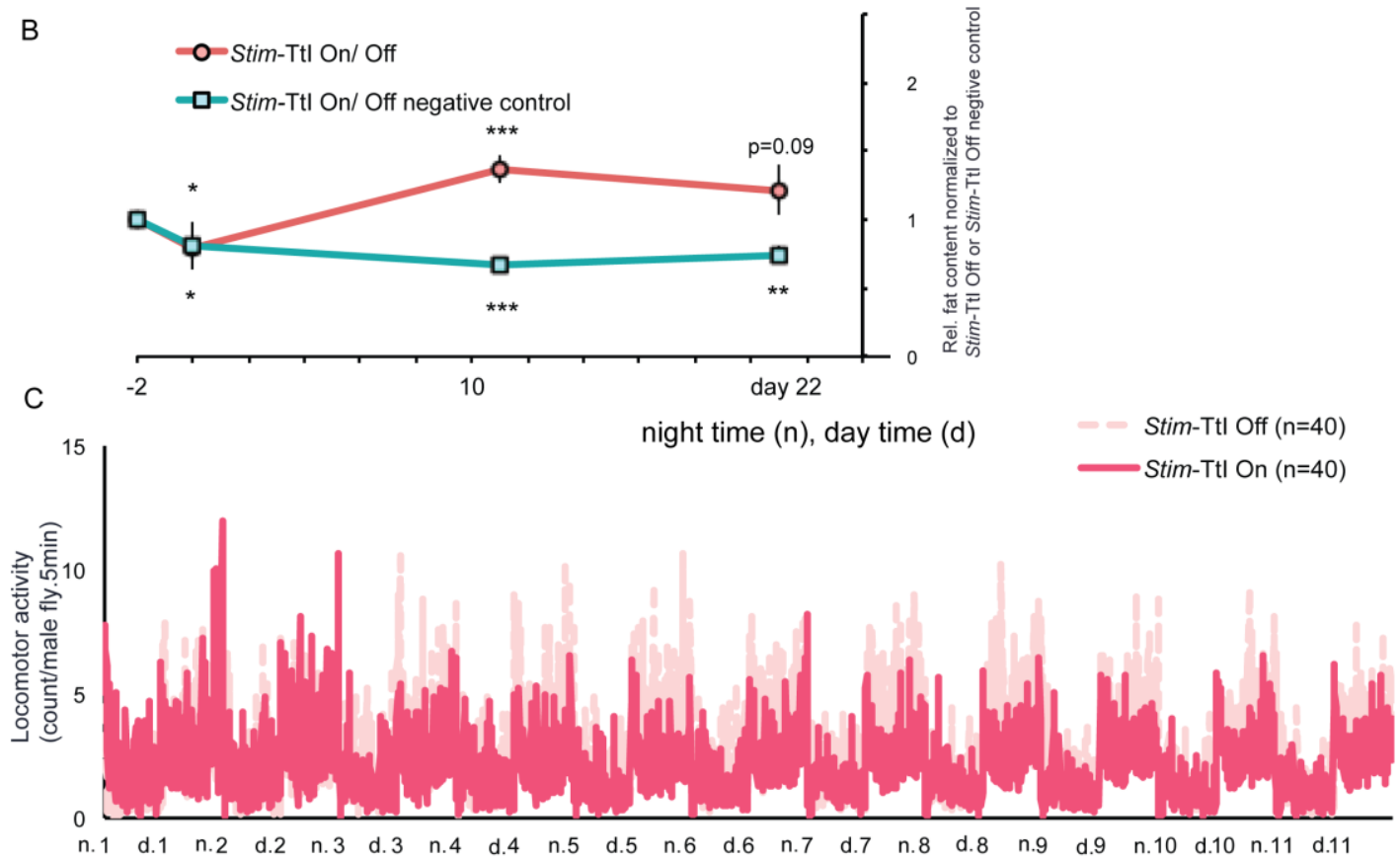

$\mathrm{D}$

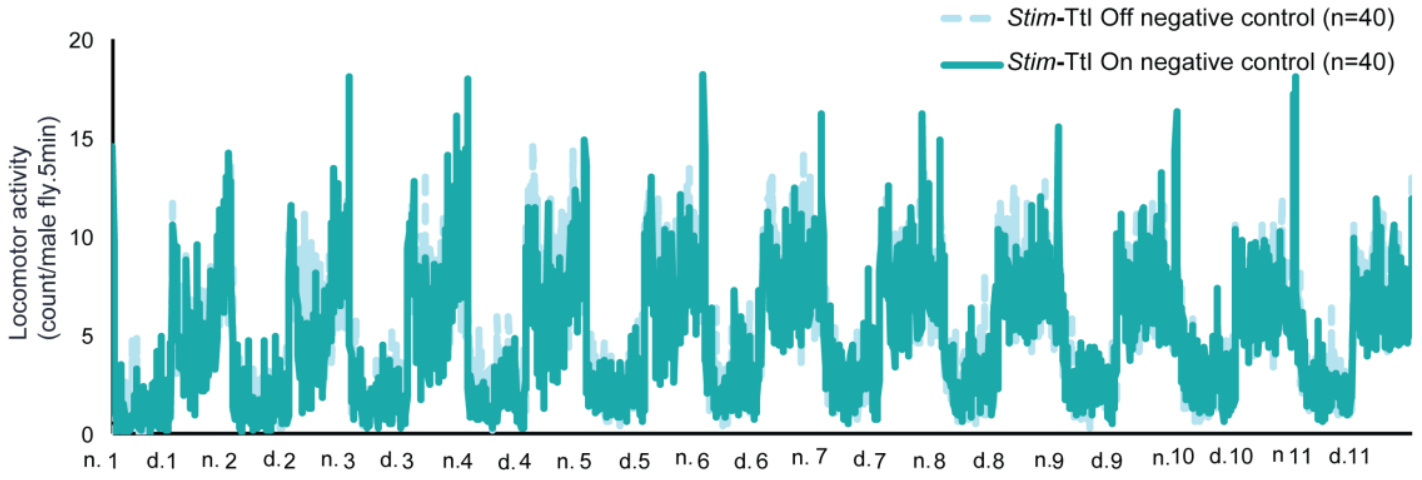

Figure 23. (Supplementary data) Stim genomic localization, Stim RNAi target region and locomotor activity counts per $5 \mathrm{~min}$ of adult male flies over the 11 days after StimTtl

A. Gene scheme showing the Stim gene genome localization (X chromosome: $15,934,400$ 15,940,500), its four transcripts (Stim-RD, RF, RA, and RG), transcripts target region of StimRNAi1\&2 and Stim-RNAi3, which is based on information from fly base (http://flybase.org/). The figure is related to Figure 5. 
B. Quantification of the body fat contents of adult male flies at day 0, day 10 and day 21 after $38 \mathrm{~h}$ induction of Stim-Transient temperature-shift induced RNAi expression (Stim-TtI On) (using independent Stim RNAi3) or Stim-Ttl On negative control as compared to Stim-Ttl Off or Stim-Ttl Off negative control flies. Note the long-term body fat storage increase is observed. Relative (rel.) fat content is represented as fold change: TAG equivalent content/protein content of Stim-TtI On or Stim-Ttl On negative control normalized with respect to the TAG equivalent content/protein content of Stim-TI Off or Stim-TI Off negative control flies serving as the respective controls. The Stim RNAi3 transgene line was used in this study. Error bars represent the standard deviation of samples. ${ }^{*}=p<0.05,{ }^{* *}=p<0.01,{ }^{* \star *}=$ $\mathrm{p}<0.001$ (Student's t-test). The figure is related to Figure 5 .

C. D. Real-time locomotor activities of adult male flies from day 1 to day 11 after Stim-TtI On indicates that day-time locomotor activity of Stim-TI On/Off, Stim-TI On negative control / Stim-Off negative control flies (C), while Stim-TI On negative control flies was not affected as compared to the corresponding control (Stim-Ttl Off negative control) flies (D).

Stim RNAi1 transgene line was used in this study. The figure is related to main Figure 11D.

A
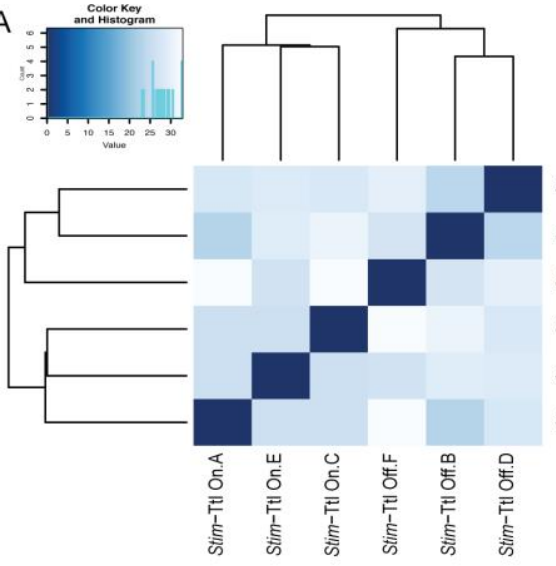

C

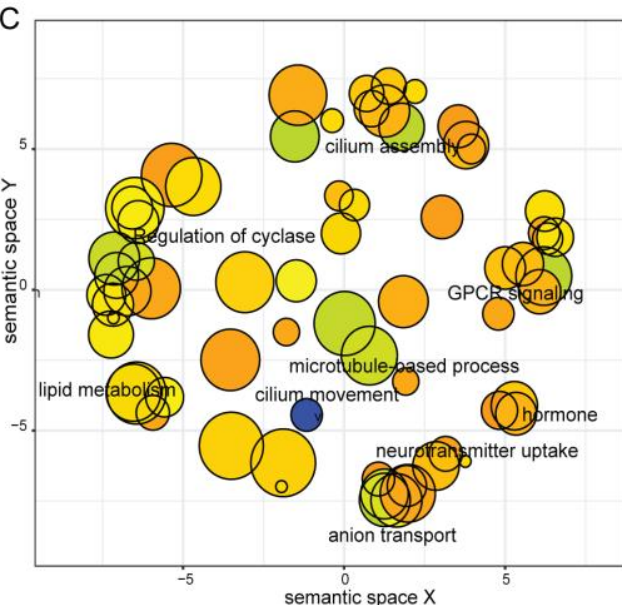

B
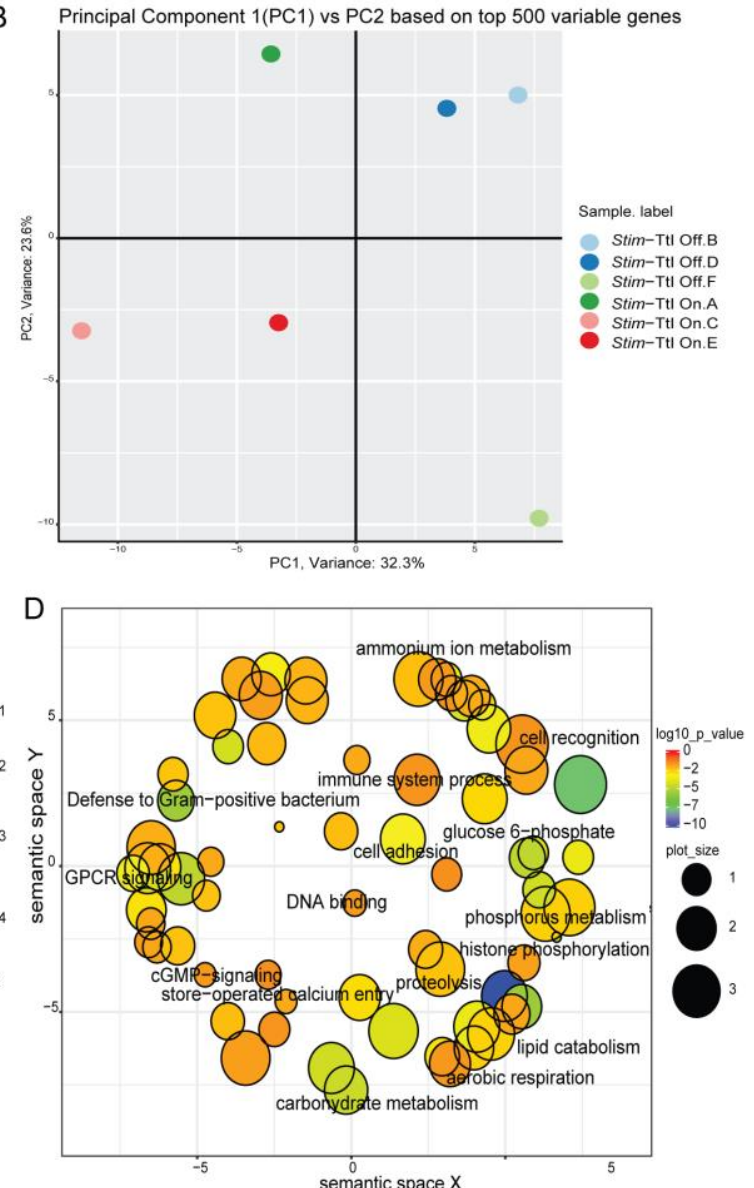

Figure 24. (Supplementary data) Sample variance analysis of RNA-seq data, and Gene Ontology analysis (biological process) of up and down-regulated genes

A. Heat map by DESeq2 shows Euclidean distances among the six RNA-seq sample data sets: Stim-Ttl On.A, Stim-Ttl Off.B, Stim-Ttl On.C, Stim-Ttl Off.D, Stim-Ttl On.E, Stim-Ttl Off.F. Note that Stim-Ttl On group samples (Stim-Ttl On.A, Stim-Ttl On.C, Stim-Ttl On.E), 
and Stim-TI Off group samples (Stim-Ttl Off.B, Stim-Ttl Off.D, Stim-TtI Off.F) are more closely clustered, respectively.

B. Principal Component Analysis (PCA) plot was analysed with DESeq2 based on top 500 most differential regulated genes of 6 samples' data sets: Stim-Ttl On.A (light blue), Stim-Tt। Off.B (deep blue), Stim-TtI On.C (light green), Stim-Ttl Off.D (deep green), Stim-TtI On.E (light red), Stim-Ttl Off.F (deep red) are shown on two-dimentional plane by color dot according to their first two principle components. There is clear separation between Stim-TtI On (Stim-Ttl On.A, Stim-Ttl On.C, Stim-Ttl On.E) and Stim-Ttl Off (Stim-Ttl Off.B, Stim-Ttl Off.D, Stim-Ttl Off.F) group samples, although the large variances among samples belong to the same group exist.

C. Biological process Gene Ontology term analysis ( $p$ value $<0.05$ ) shows the up-regulated gene groups which encode proteins get involved in the following biological processes: cilium assembly, regulation of cyclase activity, GPCR signaling, lipid metabolic processes, microtubule-based process, cilia movement, regulation of hormone, neurotransmitter uptake, anion transport. D. The down-regulated gene groups which encode proteins get involved in the following biological processes: the ammonium ion metabolic process, the cell recognition, immune system process, defense to Gram-positive bacterium, glucose phosphate, cell adhesion, GPCR signaling, DNA binding, phosphorus metabolism, histone phosphorylation, cGMP signaling, store-operated calcium entry, proteolysis, lipid catabolic process, aerobic respiration, carbohydrate metabolic process. Note that each circle represents one GO term; color code indicated $p$ value, the scale is defined by $\log _{10}(p$ values); circle size indicates the gene numbers in each $\mathrm{GO}$ term, the scale is defined by $\log _{10}$ (gene numbers in each GO term). Stim RNAi1 transgene line was used in RNA-seq studies. The figure is related to Figure 14. 

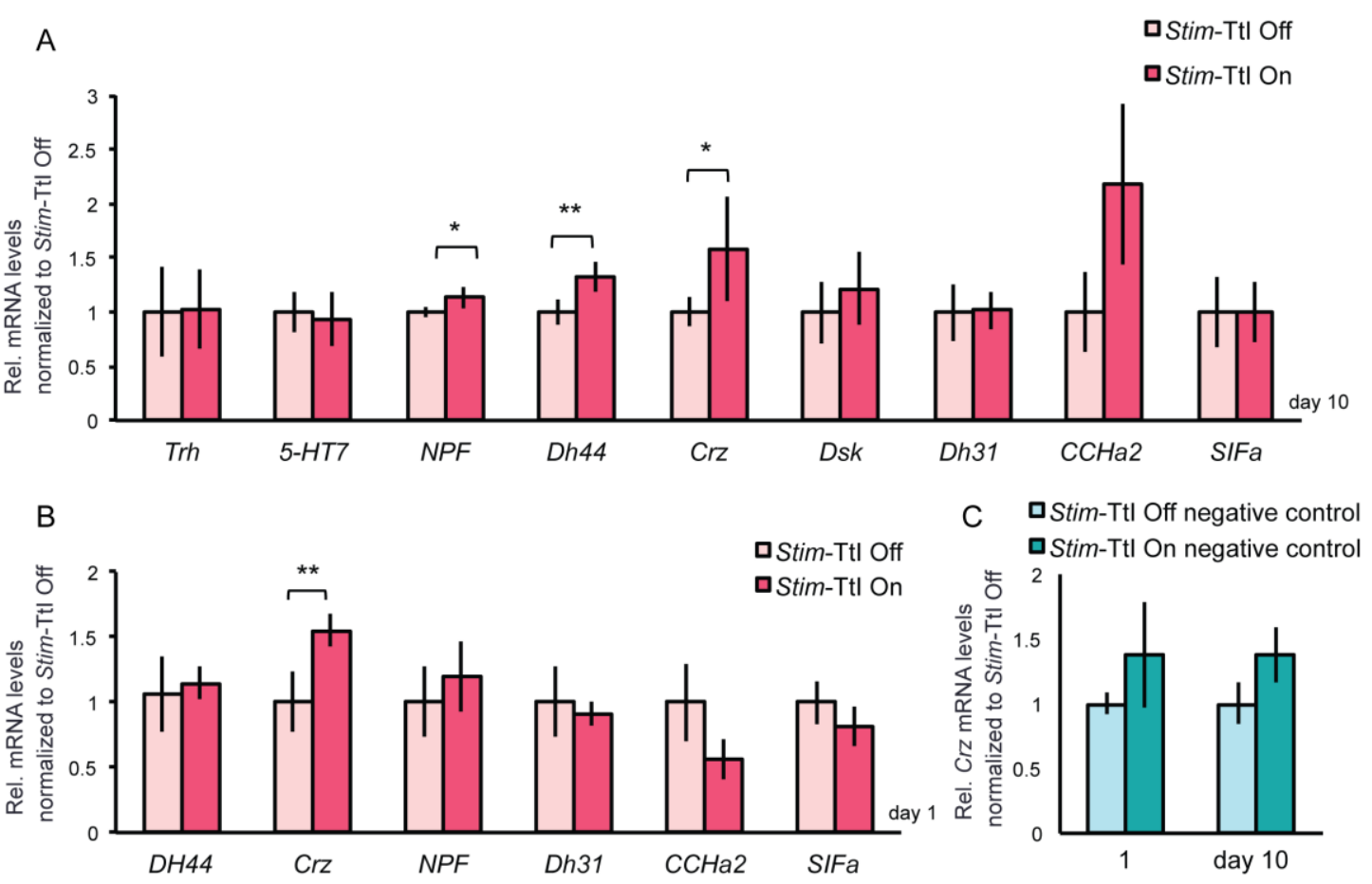

Figure 25. (Supplementary data) Gene expression level changes of food intake-related neuropeptide and neurotransmitter signaling genes in the heads of adult male flies after Stim-TtI

A. B. RT-qPCR analysis of adult male heads tissue indicates the mRNA expression of food intake related neuropeptide or neurotransmitter genes Trh, 5-HT7, NPF, Dh44, Crz, Dsk, Dh31, CCHa2, SIFa at day 10 and Dh44, Crz, NPF, Dh31, CCHa2, SIFa at day 1 after StimTtl On as compared to the corresponding control (Stim-Ttl Off) flies. A. Note that NPF, Dh44, Crz's mRNA expressions levels of day 10 Stim-Ttl On fly head tissue was significantly increased. B. Crz's mRNA expression of day 1 Stim-Ttl On fly heads was significantly increased as compared to corresponding control (Stim-Ttl Off) flies. Relative (Rel.) mRNA levels are represented as fold change, which refers to the basis value $(=1)$ obtained from Stim-Ttl Off flies Stim-Ttl Off; Act5C mRNA served an internal standard and was used as reference to normalize the levels of above mentioned gene mRNA expression.

C. RT-qPCR analysis of adult male heads tissue indicates that mRNA expression levels of food intake related neuropeptide genes Crz at day 1 and day 10 Stim-TI On negative control flies appears to be increased as compared to the corresponding control (Stim-Ttl Off) flies. Quantification of gene relative (Rel.) mRNA levels refers to method described in A.

Stim RNAi1 transgene line was used in above studies. Error bars represent the standard deviation of samples. ${ }^{*}=p<0.05,{ }^{* *} ;=p<0.01,{ }^{* * *}=p<0.001$ (Student's t-test). This figure is related to Figure 15. 

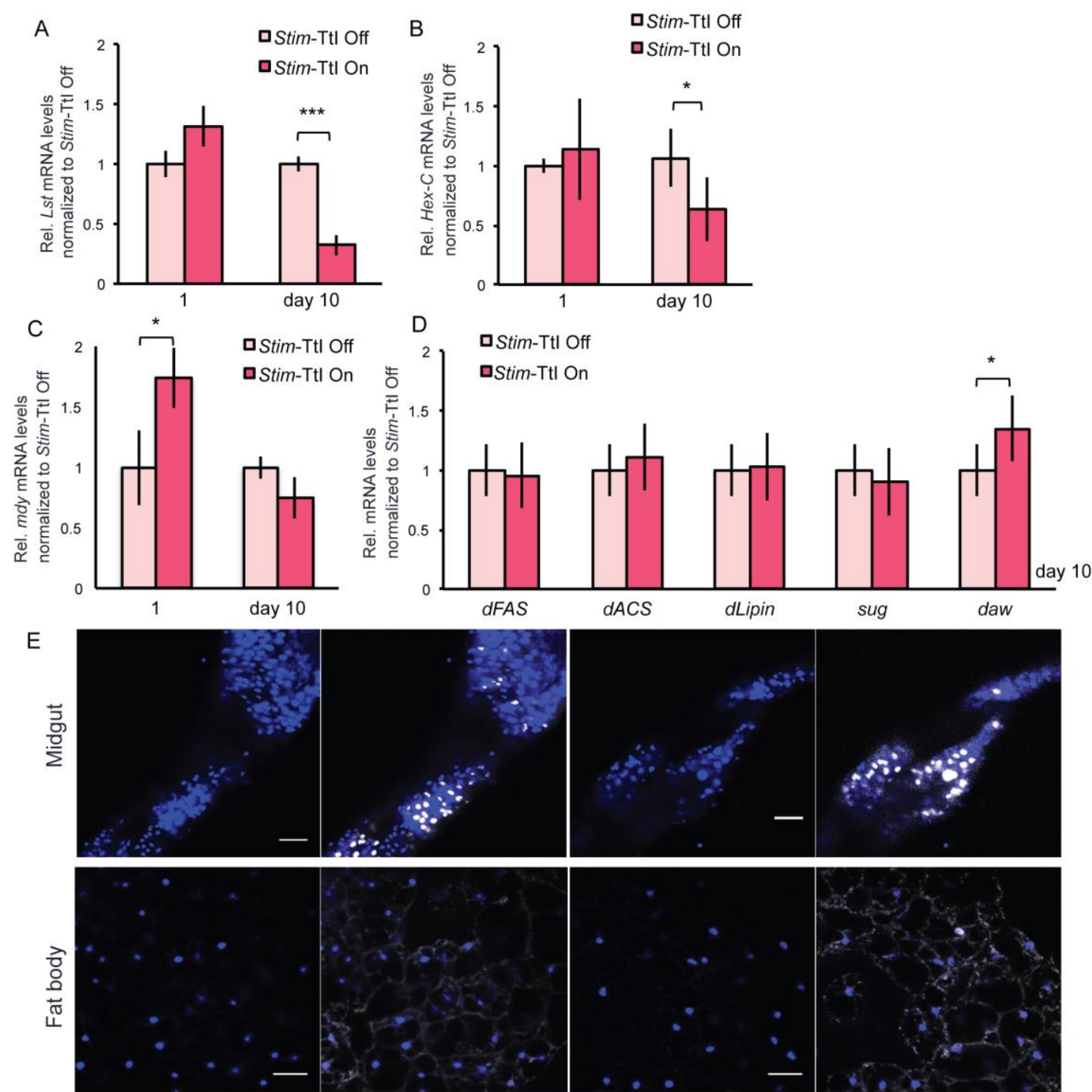

day 10 Stim-Ttl Off

EdU/Nuclei

day 10 Stim-Ttl On

F
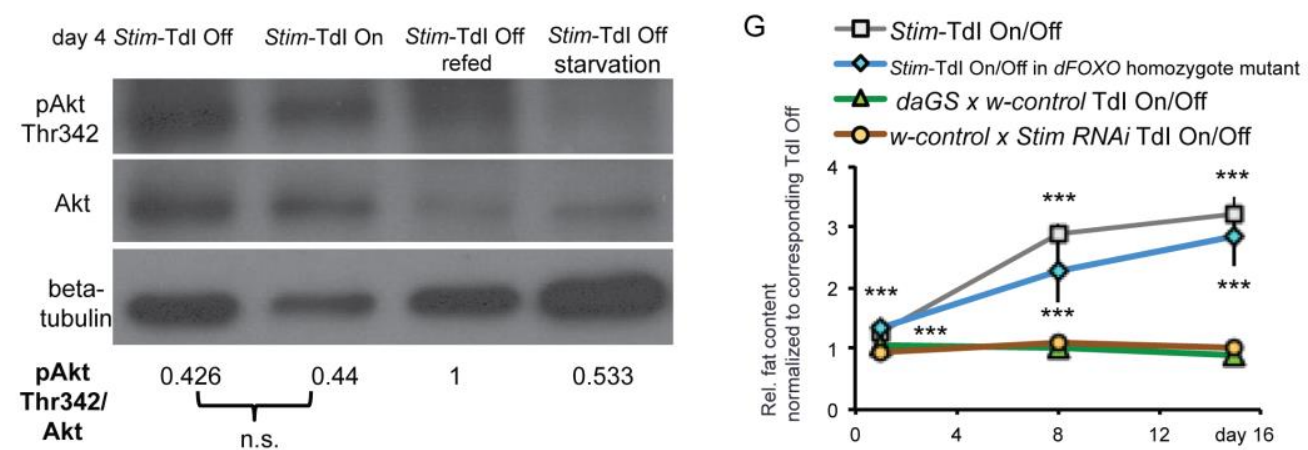

Figure 26. (Supplementary data) Selective insulin resistance occurs in peripheral tissues of LISO adult males

A. RT-qPCR analysis of male fat body tissue indicates that (B) the mRNA expression of the insulin secretion-suppressing gene Limostatin (Lst) and a homolog gene (Hex-C) encoding the mammalian glycolytic enzyme Glucokinase (GCK) was not affected at day 0 , substantially down-regulated at day 10; but (C) the mRNAs expression of the lipid biosynthesis related gene mdy was significantly increased at day 1 and reduced to normal level at day 10; (D) other lipid biosynthesis related genes dFAS, ACS, dLipin, sugarbabe (sug) are not 
significantly affected, except that the mRNA expression of Activin-like ligand (transformation growth factor $\beta$ familiy) gene daw was significantly up-regulated at day 10 after StimTransient temperature-shift induced RNAi expression (Stim-TtI On) as compared to the corresponding control (Stim-Ttl Off) flies. Quantification of gene Relative (Rel.) mRNA levels are represented as fold change, which refers to the basis value $(=1)$ obtained from Stim-TtI Off flies; Act5C mRNA served an internal standard and was used as reference to normalize the levels of above mentioned gene mRNA expression.

E. After six days EdU food feeding, EdU and Hoechst staining of midgut tissues (as positive control) and adult male fat body tissues of day 10 Stim-TtI On and Stim-TtI Off adult males. Hoechst indicates localizations of nuclei, while EdU signals indicate the nuclei ever experienced new DNA synthesis, which is required for cell proliferation. Unlike a host of EdU positive nuclei can be found in proliferation active midgut tissues of day $10 \mathrm{Stim}$-TtI On and Stim-Ttl Off flies, EdU staining in fat body tissue indicates the new DNA synthesis required by proliferation was not significantly increased at day 10 after Stim-TtI On as compared to the corresponding control (Stim-Ttl Off) flies. For details of EdU staining see 2.4.4.

F. Western blot analysis showing the accumulation of activated Akt (pAkt Thr342; Thr342 phosphorylation of Akt), Akt and the reference protein Tubulin in the day 4 adult male peripheral tissues (fly tissues without head) after Stim-Tdl On and corresponding control (Stim-Tdl Off) flies, and their accumulation in Stim-Tdl Off males with (24 hours starvation, and re-fed with $10 \%$ sugar food) and with (24 hours starvation) food supply. Note that pAkt Thr342 was not significantly affected in response to the earlier Stim-Tdl On as observed with fed versus starved control flies. Western blott on abdomens of day 4 Stim-Tdl On adult male flies indicates that activated Akt (Thr342 phosphorylation of Akt normalized to Akt) remained same level as compared to value of corresponding Stim-Tdl Off flies; Correspondent ratio value of pAkt/Akt levels based on integrated intensity was showed below the bands using the value of Stim-Tdl Off (refed) flies as reference.

H. Quantification of the body fat content at day 1, 8, and 15 after Stim-Tdl On in adult male flies as compared to corresponding control (Stim-Tdl Off) flies and Tdl On in two kinds of negative control ( $w$-control $x$ Stim RNAi 1 as Tdl On negative control 1 ; w-control $x$ daGS as Tdl On negative control 2) adult male flies as compared to corresponding Tdl Off negative control flies. Stim-Tdl flies were either wild type background or homozygous dFOXO mutant males. Note that the obesity phenotype develops after Stim-Tdl On in both wild type and homozygote dFOXO mutant. Note also that drug feeding ( $T d l$ On) did not cause the long-term body fat content increase in two kinds of negative control flies. Relative (rel.) fat content is represented as fold change: TAG equivalent content/protein content of Stim-Tdl On or Tdl On flies normalized with respect to the TAG equivalent content/protein content of corresponding Stim-Tdl Off or Tdl Off flies (serving as reference). Stim RNAi1 transgene line was used in following studies. Error bars represent the standard deviation of samples. ${ }^{*}=p<0.05,{ }^{* *} ;=$ $p<0.01,{ }^{* * *}=p<0.001$ (Student's t-test). This figure is related to Figure 16. 


\section{References}

Adams, M.D., Celniker, S.E., Holt, R.A., Evans, C.A., Zhao, Q., Spradling, A.C., Mattei, B., An, H.J., Weinstock, G.M., Holt, R.A., et al. (2000). The genome sequence of Drosophila melanogaster. Science 287, 2185-2195.

Agrawal, N., Delanoue, R., Mauri, A., Basco, D., Pasco, M., Thorens, B., and Le'opold, P. (2016). The Drosophila TNF Eiger is an adipokine that acts on insulin-producing cells to mediate nutrient response. Cell Metab. 23, 675-684.

Al-Anzi, B., Sapin, V., Waters, C., Zinn, K., Wyman, R.J., and Benzer, S. (2009). Obesityblocking neurons in Drosophila. Neuron 63, 329-341.

Albin, S.D., Kaun, K.R., Knapp, J.M., Chung, P., Heberlein, U., and Simpson, J.H. (2015). A subset of serotonergic seurons evokes hunger in adult Drosophila. Curr. Biol. 25, 2435-2440.

Alfa, R.W., and Kim, S.K. (2016). Using Drosophila to discover mechanisms underlying type 2 diabetes. Dis. Model. Mech. 9, 365-376.

Alfa, R.W., Park, S., Skelly, K.-R., Poffenberger, G., Jain, N., Gu, X., Kockel, L., Wang, J., Liu, Y., Powers, A.C., et al. (2015). Suppression of insulin production and secretion by a decretin hormone. Cell Metab. 21, 323-333.

Alic, N., Giannakou, M.E., Papatheodorou, I., Hoddinott, M.P., Andrews, T.D., Bolukbasi, E., and Partridge, L. (2014). Interplay of dFOXO and two ETS-family transcription factors determines lifespan in Drosophila melanogaster. PLoS Genet. 10, e1004619.

Alpatov, W. (1932). Egg production in Drosophila melanogaster and some factors which influence it. J. Exp. Zool. 63, 85-111.

Altarejos, J.Y., and Montminy, M. (2011). CREB and the CRTC co-activators: sensors for hormonal and metabolic signals. Nat. Rev. Mol. Cell Biol. 12, 141-151.

Amaya, M.J., and Nathanson, M.H. (2013). Calcium signaling in the Liver. Compr. Phsiol. 3, 515-539.

Ameer, F., Scandiuzzi, L., Hasnain, S., Kalbacher, H., and Zaidi, N. (2014). De novo lipogenesis in health and disease. Metabolism. 63, 895-902.

Apidianakis, Y., and Rahme, L.G. (2011). Drosophila melanogaster as a model system for human brain cancers. Dis. Model. Mech. 4, 21-30.

Del Arco, A., Agudo, M., and Satrústegui, J. (2000). Characterization of a second member of the subfamily of calcium-binding mitochondrial carriers expressed in human non-excitable tissues. Biochem. J. 345, 725-732.

Ariotti, N., Murphy, S., Hamilton, N.A., Wu, L., Green, K., Schieber, N.L., Li, P., Martin, S., and Parton, R.G. (2012). Postlipolytic insulin-dependent remodeling of micro lipid droplets in adipocytes. Mol. Biol. Cell 23, 1826-1837.

Arner, P., and Langin, D. (2014). Lipolysis in lipid turnover, cancer cachexia, and obesityinduced insulin resistance. Trends Endocrinol. Metab. 25, 255-262. 
Arora, G.K., Tran, S.L., Rizzo, N., Jain, A., and Welte, M.A. (2016). Temporal control of bidirectional lipid-droplet motion depends on the ratio of kinesin-1 and its cofactor Halo. J. Cell Sci. 129, 1416-1428.

Arrese, E.L., and Soulages, J.L. (2010). Insect fat body: energy, metabolism, and regulation. Annu. Rev. Entomolgy 55, 207-225.

Arrese, E.L., and Wells, M.A. (1994). Purification and properties of a phosphorylatable triacylglycerol lipase from the fat-body of an insect, Manduca Sexta. J. Lipid Res. 35, 16521660.

Arrese, E.L., Flowers, M.T., Gazard, J.L., and Wells, M.A. (1999). Calcium and cAMP are second messengers in the adipokinetic hormone-induced lipolysis of triacylglycerols in Manduca sexta fat body. J. Lipid Res. 40, 556-564.

Arrese, E.L., Rivera, L., Hamada, M., Mirza, S., Hartson, S.D., Weintraub, S., and Soulages, J.L. (2008). Function and structure of lipid storage droplet protein 1 studied in lipoprotein complexes. Arch. Biochem. Biophys. 473, 42-47.

Arruda, A.P., and Hotamisligil, G.S. (2015). Calcium homeostasis and organelle function in the pathogenesis of obesity and diabetes. Cell Metab. 22, 381-397.

Attrill, H., Falls, K., Goodman, J.L., Millburn, G.H., Antonazzo, G., Rey, A.J., and Marygold, S.J. (2016). Flybase: establishing a gene group resource for Drosophila melanogaster. Nucleic Acids Res. 44, D786-D792.

Bandini, L.G., Schoeller, D.A., Cyr, H.N., and Dietz, W.H. (1990). Validity of reported energy intake in obese and nonobese adolescents. Am. J. Clin. Nutr. 52, 421-425.

Banerjee, K.K., Ayyub, C., Sengupta, S., and Kolthur-Seetharam, U. (2012). dSir2 deficiency in the fatbody, but not muscles, affects systemic insulin signaling, fat mobilization and starvation survival in flies. Aging (Albany. NY). 4, 206-223.

Barry, W.E., and Thummel, C.S. (2016). The Drosophila HNF4 nuclear receptor promotes glucose-stimulated insulin secretion and mitochondrial function in adults. Elife 5, e11183.

Barsh, G.S., Farooqi, I.S., and O'Rahilly, S. (2000). Genetics of body-weight regulation. Nature 404, 644-651.

Bauer, R., Voelzmann, A., Breiden, B., Schepers, U., Farwanah, H., Hahn, I., Eckardt, F., Sandhoff, K., and Hoch, M. (2009). Schlank, a member of the ceramide synthase family controls growth and body fat in Drosophila. EMBO J. 28, 3706-3716.

Baumbach, J. (2014). PhD thesis: An in vivo RNAi screen identifies evolutionary conserved Drosophila fat storage regulators. Georg-August University Göttingen.

Baumbach, J., Hummel, P., Bickmeyer, I., Kowalczyk, K.M., Frank, M., Knorr, K., Hildebrandt, A., Riedel, D., Jäckle, H., and Kühnlein, R.P. (2014a). A Drosophila in vivo screen identifies store-operated calcium entry as a key regulator of adiposity. Cell Metab. 19, 331-343.

Baumbach, J., Xu, Y., Hehlert, P., and Kühnlein, R.P. (2014b). Gaq, GY1 and PIc21C control 
Drosophila body fat storage. J. Genet. Genomics 41, 283-292. doi:10.1016/j.jgg.2014.03.005.

Bays, H. (2014). Central obesity as a clinical marker of adiposopathy; increased visceral adiposity as a surrogate marker for global fat dysfunction. Curr. Opin. Endocrinol. Diabetes Obes. 21, 345-351.

Becnel, J., Johnson, O., Luo, J., Nässel, D.R., and Nichols, C.D. (2011). The serotonin 5HT7dro receptor is expressed in the brain of Drosophila, and is essential for normal courtship and mating. PLoS One 6, e20800.

Bell, C.G., Walley, A.J., and Froguel, P. (2005). The genetics of human obesity. Nat. Rev. Genet. 6, 221-234.

Bellen, H.J., Tong, C., and Tsuda, H. (2010). 100 years of Drosophila research and its impact on vertebrate neuroscience: a history lesson for the future. Neuroscience $11,514-522$.

Beller, M., Bulankina, A. V., Hsiao, H.H., Urlaub, H., Jäckle, H., and Kühnlein, R.P. (2010). PERILIPIN-dependent control of lipid droplet structure and fat storage in Drosophila. Cell Metab. 12, 521-532.

Bellisari, A. (2008). Evolutionary origins of obesity. Obes. Rev. 9, 165-180.

Benzer, S. (1967). Behavioral mutants of Drosophila isolated by countercurrent distribution. Proc. Natl. Acad. Sci. U. S. A. 58, 1112-1119.

Berg, J., Tymoczko, J., and Stryer, L. (2002). Biochemistry. 5th Edition (New York: W H Freeman).

Bernardo Le'o Wajchenberg (2000). Subcutaneous and visceral adipose tissue : their relation to the metabolic syndrome. Endocr. Rev. 21, 697-738.

Berridge, M.J. (2009). Inositol trisphosphate and calcium signalling mechanisms. Biochim. Biophys. Acta - Mol. Cell Res. 1793, 933-940.

Berridge, M.J., Lipp, P., and Bootman, M.D. (2000). The versatility and universality of calcium signalling. Nat. Rev. Mol. Cell Biol. 1, 11-21.

Berridge, M.J., Bootman, M.D., and Roderick, H.L. (2003). Calcium signalling: dynamics, homeostasis and remodelling. Nat. Rev. Mol. Cell Biol. 4, 517-529.

Berrigan, D., and Partridge, L. (1997). Influence of temperature and activity on the metabolic rate of adult Drosophila melanogaster. Comp. Biochem. Physiol. A. Physiol. 118, 1304.

Bharucha, K.N., Tarr, P., and Zipursky, S.L. (2008). A glucagon-like endocrine pathway in Drosophila modulates both lipid and carbohydrate homeostasis. J. Exp. Biol. 211, 31033110.

Bhatia, R., Lesser, D.J., Oliveira, F.G.S.A., Tran, W.H., Keens, T.G., Khoo, M.C.K., and Ward, S.L.D. (2015). Body fat composition: a predictive factor for sleep related breathing disorder in obese children. J. Clin. Sleep Med. 11, 1039-1045.

Bi, J., Xiang, Y., Chen, H., Liu, Z., Gronke, S., Kuhnlein, R.P., and Huang, X. (2012). 
Opposite and redundant roles of the two Drosophila perilipins in lipid mobilization. J. Cell Sci. 125, 3568-3577.

Bi, J., Wang, W., Liu, Z., Hueng, X., Jiang, Q., Liu, G., Wang, Y., and Huang, X. (2014a). Seipin promotes adipose tissue fat storage through the ER $\mathrm{Ca}^{2+}$-ATPase SERCA. Cell Metab. 19, 861-871.

Bi, X., Seabolt, L., Shibao, C., Buchowski, M., Kang, H., Keil, C., Tyree, R., and Silver, H. (2014b). DXA-measured visceral adipose tissue predicts impaired glucose tolerance and metabolic syndrome in obese Caucasian and African-American women. Eur. J. Clin. Nutr. 69, 329-336.

Billi, A.C., Fischer, S.E.J., and Kim, J.K. (2014). Endogenous RNAi pathways in C. elegans. WormBook, Ed. C. Elegans Res. Community, WormBook, 10.189, 1-49.

Birse, R.T., Choi, J., Reardon, K., Rodriguez, J., Graham, S., Diop, S., Ocorr, K., Bodmer, R., and Oldham, S. (2010). High-fat-diet-induced obesity and heart dysfunction are regulated by the TOR pathway in Drosophila. Cell Metab. 12, 533-544.

Bischof, J., Maeda, R.K., Hediger, M., Karch, F., and Basler, K. (2007). An optimized transgenesis system for Drosophila using germ-line-specific phiC31 integrases. Proc. Natl. Acad. Sci. U. S. A. 104, 3312-3317.

Bjørndal, B., Burri, L., Staalesen, V., Skorve, J., and Berge, R.K. (2011). Different adipose depots: their role in the development of metabolic syndrome and mitochondrial response to hypolipidemic agents. J. Obes. 490650.

Bjursell, M., and Gerdin, A. (2008). Acutely reduced locomotor activity is a major contributor to Western diet-induced obesity in mice. Am. J. Physiol. Endocrinol. Metab. 294, E251-E260.

Blackstone, R.P. (2016). Obesity: the medical practitioner's essential guide (Springer International Publishing Switzerland).

Bland, M.L., Lee, R.J., Magallanes, J.M., Foskett, J.K., and Birnbaum, M.J. (2010). AMPK supports growth in Drosophila by regulating muscle activity and nutrient uptake in the gut. Dev. Biol. 344, 293-303.

Blundell, J.E., Caudwell, P., Gibbons, C., Hopkins, M., Naslund, E., King, N., and Finlayson, G. (2012). Role of resting metabolic rate and energy expenditure in hunger and appetite control: a new formulation. Dis. Model. Mech. 5, 608-613.

Blundell, J.E., Gibbons, C., Caudwell, P., Finlayson, G., and Hopkins, M. (2015). Appetite control and energy balance: impact of exercise. Obes. Rev. 16, 67-76.

Böhni, R., Riesgo-Escovar, J., Oldham, S., Brogiolo, W., Stocker, H., Andruss, B.F., Beckingham, K., and Hafen, E. (1999). Autonomous control of cell and organ size by CHICO, a Drosophila homolog of vertebrate IRS1-4. Cell 97, 865-875.

Bootman, M.D. (2012). Calcium Signaling. Cold Spring Harb. Perspect. Biol. 4, a011171.

Borcherding, A.F. (2014). Bachelor thesis: A genetic screen identifies candidate genes which lead to programmed obesity by transient Knock Down in Drosophila. Georg-August University 
Göttingen.

Borsini, F., Bendotti, C., Thurlby, P., and Samanin, R. (1982). Evidence that systemically administered salbutamol reduces food intake in rats by actiing on central beta-adrenergic sites. Life Sci. 30, 905-911.

Boucher, J., Kleinridders, A., and Kahn, C.R. (2014). Insulin receptor signaling in normal and insulin-resistant states. Cold Spring Harb. Perspect. Biol. 6, a009191.

Braco, J.T., Gillespie, E.L., Alberto, G.E., Brenman, J.E., and Johnson, E.C. (2012). Energydependent modulation of glucagon-like signaling in Drosophila via the AMP-activated protein kinase. Genetics 192, 457-466.

Brand, A.H., and Perrimon, N. (1993). Targeted gene expression as a means of altering cell fates and generating dominant phenotypes. Development 118, 401-415.

Brankatschk, M., and Eaton, S. (2010). Lipoprotein particles cross the blood-brain barrier in Drosophila. J. Neurosci. 30, 10441-10447.

Brasaemle, D.L., Rubin, B., Harten, I.A., Gruia-Gray, J., Kimmel, A.R., and Londos, C. (2000). Perilipin $A$ increases triacylglycerol storage by decreasing the rate of triacylglycerol hydrolysis. J. Biol. Chem. 275, 38486-38493.

Bray, G.A., and Popkin, B.M. (1998). Dietary fat intake does affect obesity! Am. J. Clincal Nutr. 68, 1157-1173.

Brini, M., Calì, T., Ottolini, D., and Carafoli, E. (2013). The plasma membrane calcium pump in health and disease. FEBS J. 280, 5385-5397.

Britton, K.A., Massaro, J.M., Murabito, J.M., Kreger, B.E., Hoffmann, U., and Fox, C.S. (2013). Body fat distribution, incident cardiovascular disease, cancer, and all-cause mortality. J. Am. Coll. Cardiol. 62, 921-925.

Brogiolo, W., Stocker, H., Ikeya, T., Rintelen, F., Fernandez, R., and Hafen, E. (2001). An evolutionarily conserved function of the Drosophila insulin receptor and insulin-like peptides in growth control. Curr. Biol. 11, 213-221.

Brown, M.R., Crim, J.W., Arata, R.C., Cai, H.N., Chun, C., and Shen, P. (1999). Identification of a Drosophila brain-gut peptide related to the neuropeptide $Y$ family. Peptides 20, 10351042.

Brunet, A., Sweeney, L.B., Sturgill, J.F., Chua, K.F., Greer, P.L., Lin, Y., Tran, H., Ross, S.E., Mostoslavsky, R., Cohen, H.Y., et al. (2004). Stress-dependent regulation of FOXO transcription factors by the SIRT1 deacetylase. Science 303, 2011-2015.

Buch, S., Melcher, C., Bauer, M., Katzenberger, J., and Pankratz, M.J. (2008). Opposing effects of dietary protein and sugar regulate a transcriptional target of Drosophila insulin-like peptide signaling. Cell Metab. 7, 321-332.

Buchon, N., Silverman, N., and Cherry, S. (2014). Immunity in Drosophila melanogaster from microbial recognition to whole-organism physiology. Nat. Rev. Immunol. 14, 796-810. 
Bühler, M., and Moazed, D. (2007). Transcription and RNAi in heterochromatic gene silencing. Nat. Struct. Mol. Biol. 14, 1041-1048.

Buszczak, M., Lu, X., Segraves, W.A., Chang, T.Y., and Cooley, L. (2002). Mutations in the midway gene disrupt a Drosophila acyl coenzyme A: Diacylglycerol acyltransferase. Genetics 160, 1511-1518.

Cabrero, P., Radford, J.C., Broderick, K.E., Costes, L., Veenstra, J.A., Spana, E.P., Davies, S.A., and Dow, J.A.T. (2002). The Dh gene of Drosophila melanogaster encodes a diuretic peptide that acts through cyclic AMP. J. Exp. Biol. 205, 3799-3807.

Canto, C., and Auwerx, J. (2012). Targeting sirtuin 1 to improve metabolism: all you need is NAD(+)? Pharmacol. Rev. 64, 166-187.

Carrasco, S., and Mérida, I. (2007). Diacylglycerol, when simplicity becomes complex. Trends Biochem. Sci. 32, 27-36.

Carvalho, M., Sampaio, J.L., Palm, W., Brankatschk, M., Eaton, S., and Shevchenko, A. (2012). Effects of diet and development on the Drosophila lipidome. Mol. Syst. Biol. 8, 600.

Castañeda, T.R., Jürgens, H., Wiedmer, P., Pfluger, P., Diano, S., Horvath, T.L., TangChristensen, M., and Tschöp, M.H. (2005). Obesity and the neuroendocrine control of energy homeostasis: the role of spontaneous locomotor activity. J. Nutr. 135, 1314-1319.

Cavanaugh, D.J., Geratowski, J.D., Wooltorton, J.R.A., Spaethling, J.M., Hector, C.E., Zheng, X., Johnson, E.C., Eberwine, J.H., and Sehgal, A. (2014). Identification of a circadian output circuit for rest: activity rhythms in Drosophila. Cell 157, 689-701.

Chakraborty, R., Vepuri, V., Mhatre, S.D., Paddock, B.E., Miller, S., Michelson, S.J., Delvadia, R., Desai, A., Vinokur, M., Melicharek, D.J., et al. (2011). Characterization of a Drosophila Alzheimer's disease model: pharmacological rescue of cognitive defects. PLoS One 6. e20799.

Chartier, A., Klein, P., Pierson, S., Barbezier, N., Gidaro, T., Casas, F., Carberry, S., Dowling, P., Maynadier, L., Bellec, M., et al. (2015). Mitochondrial dysfunction reveals the role of mRNA poly $(A)$ tail regulation in oculopharyngeal muscular dystrophy pathogenesis. PLoS Genet. 11, e1005092.

Chatterjee, D., Katewa, S.D., Qi, Y., Jackson, S.A., Kapahi, P., and Jasper, H. (2014). Control of metabolic adaptation to fasting by dILP6-induced insulin signaling in Drosophila oenocytes. Proc. Natl. Acad. Sci. U. S. A. 111, 17959-17964.

Chen, C., Ridzon, D.A., Broomer, A.J., Zhou, Z., Lee, D.H., Nguyen, J.T., Barbisin, M., Xu, N.L., Mahuvakar, V.R., Andersen, M.R., et al. (2005). Real-time quantification of microRNAs by stem-loop RT-PCR. Nucleic Acids Res. 33, 1-9.

Chen, J., Nolte, V., and Schlötterer, C. (2015). Temperature stress mediates decanalization and dominance of gene expression in Drosophila melanogaster. PLoS Genet. 11, e1004883.

Chien, S., Reiter, L.T., Bier, E., and Gribskov, M. (2002). Homophila: human disease gene cognates in Drosophila. Nucleic Acids Res. 30, 149-151. 
Chng, W.A., Hietakangas, V., and Lemaitre, B. (2016). Physiological adaptations to sugar intake: new paradigms from Drosophila melanogaster. Trends Endocrinol. Metab. 28, 1-12.

Choi, S., Kim, W., and Chung, J. (2011). Drosophila salt-inducible kinase (SIK) regulates starvation resistance through CAMP-response element-binding protein (CREB)-regulated transcription coactivator (CRTC). J. Biol. Chem. 286, 2658-2664.

Chua, S.C., Chung, W.K., Wu-Peng, X.S., Zhang, Y., Liu, S.M., Tartaglia, L., and Leibel, R.L. (1996). Phenotypes of mouse diabetes and rat fatty due to mutations in the OB (leptin) receptor. Science 271, 994-996.

Church, T.S., Thomas, D.M., Tudor-Locke, C., Katzmarzyk, P.T., Earnest, C.P., Rodarte, R.Q., Martin, C.K., Blair, S.N., and Bouchard, C. (2011). Trends over 5 decades in U.S. occupation-related physical activity and their associations with obesity. PLoS One 6, e19657.

Clapham, D.E. (2007). Calcium Signaling. Cell 131, 1047-1058.

Clark, L., Zhang, J.R., Tobe, S., and Lange, A.B. (2006). Proctolin: a possible releasing factor in the corpus cardiacum/corpus allatum of the locust. Peptides 27, 559-566.

Clément, K., Vaisse, C., Lahlou, N., Cabrol, S., Pelloux, V., Cassuto, D., Gourmelen, M., Dina, C., Chambaz, J., Lacorte, J.M., et al. (1998). A mutation in the human leptin receptor gene causes obesity and pituitary dysfunction. Nature 392, 398-401.

Coast, G.M., Webster, S.G., Schegg, K.M., Tobe, S.S., and Schooley, D.A. (2001). The Drosophila melanogaster homologue of an insect calcitonin-like diuretic peptide stimulates VATPase activity in fruit fly malpighian tubules. J. Exp. Biol. 204, 1795-1804.

Coleman, D.L. (1978). Obese and diabetes: two mutant genes causing diabetes-obesity syndromes in mice. Diabetologia 14, 141-148.

Coleman, C.M., and Neckameyer, W.S. (2005). Serotonin synthesis by two distinct enzymes in Drosophila melanogaster. Arch. Insect Biochem. Physiol. 59, 12-31.

Colombani, J., Raisin, S., Pantalacci, S., Radimerski, T., Montagne, J., and Léopold, P. (2003). A nutrient sensor mechanism controls Drosophila growth. Cell 114, 739-749.

Comuzzie, A.G., and Allison, D.B. (1998). The search for human obesity genes. Science 280, 1374-1377.

Contreras, L., Gomez-Puertas, P., lijima, M., Kobayashi, K., Saheki, T., and Satrústegui, J. (2007). $\mathrm{Ca}^{2+}$ activation kinetics of the two aspartate-glutamate mitochondrial carriers, aralar and citrin: role in the heart malate-aspartate NADH shuttle. J. Biol. Chem. 282, 7098-7106.

Cooper, D.M.F. (2015). Store-operated $\mathrm{Ca}^{2+-e n t r y}$ and adenylyl cyclase. Cell Calcium 58, 368-375.

Cornell, R.B., and Ridgway, N.D. (2015). CTP:phosphocholine cytidylyltransferase: function, regulation, and structure of an amphitropic enzyme required for membrane biogenesis. Prog. Lipid Res. 59, 147-171.

Cosar, E., Uçok, K., Akgün, L., Köken, G., Sahin, F.K., Arioz, D.T., and Baş, O. (2008). Body 
fat composition and distribution in women with polycystic ovary syndrome. Gynecol. Endocrinol. 24, 428-432.

Cox, L.M., and Blaser, M.J. (2015). Antibiotics in early life and obesity. Nat. Rev. Endocrinol. $11,182-190$.

D'Arrigo, P., and Servi, S. (2010). Synthesis of lysophospholipids. Molecules 15, 1354-1377.

Daines, B., Wang, H., Wang, L., Li, Y., Han, Y., Emmert, D., Gelbart, W., Wang, X., Li, W., Gibbs, R., et al. (2011). The Drosophila melanogaster transcriptome by paired-end RNA sequencing. Genome Res. 21, 315-324.

van Dam, R., and Seidell, J.C. (2007). Carbohydrate intake and obesity. Eur. J. Clin. Nutr. 61, S75-S99.

Daneholt, B. (2006). Advanced information on the nobel prize in physiology or medicine 2006.

Davenport C. B. (1941). The early history of research with Drosophila. Science 93, 305-306.

Deng, H., Gerencser, A.A., and Jasper, H. (2015). Signal integration by $\mathrm{Ca}^{2+}$ regulates intestinal stem-cell activity. Nature 528, 212-217.

Després, J.P. (2012). Body fat distribution and risk of cardiovascular disease: an update. Circulation 126, 1301-1313.

Després, J.-P., and Lemieux, I. (2006). Abdominal obesity and metabolic syndrome. Nature $444,881-887$.

Dhurandhar, N. V, Schoeller, D., Brown, A.W., Heymsfield, S.B., Thomas, D., Sørensen, T.I.A., Speakman, J.R., Jeansonne, M., Allison, D.B., and Energy Balance Measurement Working Group (2015). Energy balance measurement: when something is not better than nothing. Int. J. Obes. (Lond.). 39, 1109-1113.

DiAngelo, J.R., and Birnbaum, M.J. (2009). Regulation of fat cell mass by insulin in Drosophila melanogaster. Mol. Cell. Biol. 29, 6341-6352.

Dietzl, G., Chen, D., Schnorrer, F., Su, K.-C., Barinova, Y., Fellner, M., Gasser, B., Kinsey, K., Oppel, S., Scheiblauer, S., et al. (2007). A genome-wide transgenic RNAi library for conditional gene inactivation in Drosophila. Nature 448, 151-156.

Dionne, M.S. (2014). Immune-metabolic interaction in Drosophila. Fly (Austin). 8, 75-79.

Diop, S., Bisharat-Kernizan, J., Birse, R., Oldham, S., Ocorr, K., and Bodmer, R. (2015). PGC-1/spargel counteracts High-fat-diet-induced obesity and cardiac lipotoxicity downstream of TOR and brummer ATGL lipase. Cell Rep. 10, 1572-1584.

Dirlewanger, M., Di Vetta, V., Guenat, E., Battilana, P., Seematter, G., Schneiter, P., Âquier, E.J., and Tappy, L. (2000). Effects of short-term carbohydrate or fat overfeeding on energy expenditure and plasma leptin concentrations in healthy female subjects. Int. J. Obes. 24, 1413-1418.

Dolmetsch, R.E., Lewis, R.S., Goodnow, C.C., and Healy, J.I. (1997). Differential activation of 
transcription factors induced by $\mathrm{Ca}^{2+}$ response amplitude and duration. Nature $386,855-858$.

Donini, L.M., Poggiogalle, E., Del Balzo, V., Lubrano, C., Faliva, M., Opizzi, A., Perna, S., Pinto, A., and Rondanelli, M. (2013). How to estimate fat mass in overweight and obese subjects. Int. J. Endocrinol. 2013.

Droujinine, I.A., and Perrimon, N. (2016). Interorgan communication pathways in physiology: focus on Drosophila. Annu. Rev. Genet. 50, 539-570.

Dus, M., Lai, J.S.-Y., Gunapala, K.M., Min, S., Tayler, T.D., Hergarden, A.C., Geraud, E., Joseph, C.M., Suh, G.S.B., Ai, M., et al. (2015). Nutrient sensor in the brain directs the action of the brain-gut axis in Drosophila. Neuron 87, 139-151.

Eanes, W.F., Merritt, T.J.S., Flowers, J.M., Kumagai, S., and Zhu, C.T. (2009). Direct evidence that genetic variation in glycerol-3-phosphate and malate dehydrogenase genes (Gpdh and Mdh1) affects adult ethanol tolerance in Drosophila melanogaster. Genetics 181, 607-614.

Eid, J.-P., Arias, A.M., Robertson, H., Hime, G.R., and Dziadek, M. (2008). The Drosophila STIM1 orthologue, dSTIM, has roles in cell fate specification and tissue patterning. BMC Dev. Biol. 8, 104.

Elbashir, S.M., Martinez, J., Patkaniowska, A., Lendeckel, W., and Tuschl, T. (2001). Functional anatomy of siRNAs for mediating efficient RNAi in Drosophila melanogaster embryo lysate. EMBO J. 20, 6877-6888.

English, S.B., and Butte, A.J. (2007). Evaluation and integration of 49 genome-wide experiments and the prediction of previously unknown obesity-related genes. Bioinformatics 23, 2910-2917.

Fagegaltier, D., Bougé, A.-L., Berry, B., Poisot, E., Sismeiro, O., Coppée, J.-Y., Théodore, L., Voinnet, O., and Antoniewski, C. (2009). The endogenous siRNA pathway is involved in heterochromatin formation in Drosophila. Proc. Natl. Acad. Sci. U. S. A. 106, 21258-21263.

Farooqi, I.S., and O'Rahilly, S. (2000). Recent advances in the genetics of severe childhood obesity. Arch. Dis. Child. 83, 31-34.

Faust, J.E., Verma, A., Peng, C., and Mcnew, J.A. (2012). An inventory of peroxisomal proteins and pathways in Drosophila melanogaster. Traffic 13, 1378-1392.

Feany, M.B., and Bender, W.W. (2000). A Drosophila model of Parkinson 's disease. Nature 404, 394-398.

Feng, G., Reale, V., Chatwin, H., Kennedy, K., Venard, R., Ericsson, C., Yu, K., Evans, P.D., and Hall, L.M. (2003). Functional characterization of a neuropeptide F-like receptor from Drosophila melanogaster. Eur. J. Neurosci. 18, 227-238.

Fernandez-Almonacid, R., and Rosen, O.M. (1987). Structure and ligand specificity of the Drosophila melanogaster insulin receptor. Mol. Cell. Biol. 7, 2718-2727.

Fernández-Hernández, I., Rhiner, C., and Moreno, E. (2013). Adult Neurogenesis in Drosophila. Cell Rep. 3, 1857-1865. 
Feske, S., Gwack, Y., Prakriya, M., Srikanth, S., Puppel, S.-H., Tanasa, B., Hogan, P.G., Lewis, R.S., Daly, M., and Rao, A. (2006). A mutation in Orai1 causes immune deficiency by abrogating CRAC channel function. Nature 441, 179-185.

Finucane, M.M., Stevens, G.A., Cowan, M.J., Danaei, G., Lin, J.K., Paciorek, C.J., Singh, G.M., Gutierrez, H.R., Lu, Y., Bahalim, A.N., et al. (2011). National, regional, and global trends in body-mass index since 1980: Systematic analysis of health examination surveys and epidemiological studies with 960 country-years and 9.1 million participants. Lancet 377, 557-567.

Fire, A., Xu, S., Montgomery, M.K., Kostas, S.A., Driver, S.E., and Mello, C.C. (1998). Potent and specific genetic interference by double-stranded RNA in Caenorhabditis elegans. Nature 391, 806-811.

Flegal, K.M., Kit, B.K., Orpana, H., and Graubard, B.I. (2013). Association of all-cause mortality with overweight and obesity using standard body mass index categories: a systematic review and meta-analysis. JAMA 309, 71-82.

Förster, T. (1948). Zwischenmolekulare Energiewanderung und Fluoreszenz. Ann. Phys. 33, 166-175.

Frayling, T.M., Timpson, N.J., Weedon, M.N., Zeggini, E., Freathy, R.M., Lindgren, C.M., Perry, J.R.B., Elliott, K.S., Lango, H., Rayner, N.W., et al. (2007). A common variant in the FTO gene is associated with body mass index and predisposes to childhood and adult obesity. Science 4, 889-895.

Friend, D.M., Devarakonda, K., O’Neal, T.J., Skirzewski, M., Papazoglou, I., Kaplan, A.R., Liow, J.-S., Guo, J., Rane, S.G., Rubinstein, M., et al. (2016). Basal ganglia dysfunction contributes to physical inactivity in obesity. Cell Metab. 25, 1-10.

Fuss, B., Becker, T., Zinke, I., and Hoch, M. (2006). The cytohesin Steppke is essential for insulin signalling in Drosophila. Nature 444, 945-948.

Gäde, G., and Auerswald, L. (2003). Mode of action of neuropeptides from the adipokinetic hormone family. Gen. Comp. Endocrinol. 132, 10-20.

Gáliková, M., Diesner, M., Klepsatel, P., Hehlert, P., Xu, Y., Bickmeyer, I., Predel, R., and Kühnlein, R.P. (2015). Energy homeostasis control in Drosophila adipokinetic hormone mutants. Genetics 201, 665-683. doi: 10.1534/genetics.115.178897.

Gáliková, M., Klepsatel, P., Xu, Y., and Kühnlein, R.P. (2017). The obesity-related adipokinetic hormone controls feeding and expression of neuropeptide regulators of Drosophila metabolism. Eur. J. Lipid Sci. Technol. 119. 1600138. doi:10.1002/ejlt.201600138.

Gargano, J.W., Martin, I., Bhandari, P., and Grotewiel, M.S. (2005). Rapid iterative negative geotaxis (RING): a new method for assessing age-related locomotor decline in Drosophila. Exp. Gerontol. 40, 386-395.

Garlapow, M.E., Huang, W., Yarboro, M.T., Peterson, K.R., and Mackay, T.F.C. (2015). Quantitative genetics of food intake in Drosophila melanogaster. PLoS One 10, e0138129. 
Garrow, J.S. (1988). Obesity and Related Diseases (London: Churchill Livingstone).

Géminard, C., Rulifson, E.J., and Léopold, P. (2009). Remote control of insulin secretion by fat cells in Drosophila. Cell Metab. 10, 199-207.

Gilby, A.R. (1965). Lipids and their metabolism in insects. Annu. Rev. Entomol. 10, 141-160.

Goberdhan, D.C.I., Paricio, N., Goodman, E.C., Mlodzik, M., and Wilson, C. (1999). Drosophila tumor suppressor PTEN controls cell size and number by antagonizing the Chico / PI3-kinase signaling pathway. Genes Dev. 3244-3258.

Golic, K.G., and Lindquist, S. (1989). The FLP recombinase of yeast catalyzes site-specific recombination in the Drosophila genome. Cell 59, 499-509.

Graveley, B.R., Brooks, A.N., Carlson, J.W., Duff, M.O., Landolin, J.M., Yang, L., Artieri, C.G., van Baren, M.J., Boley, N., Booth, B.W., et al. (2011). The developmental transcriptome of Drosophila melanogaster. Nature 471, 473-479.

Gray, D.S., and Fujioka, K.E.N. (1991). Use of relative weight and body mass index for the determination of adiposity. J. Clin. Epidemilogy 44, 545-549.

Green, M.R., and Joseph Sambrook (2012). Molecular Cloning: a laboratory manual (New York: John Inglis).

Greenspan, R. (2004). Fly Pushing: The theory and practice of Drosophila genetics (New York: Cold Spring Harbor Laboratory Press).

Gregor, M.F., and Hotamisligil, G.S. (2011). Inflammatory mechanisms in obesity. Annu. Rev. Immunol. 29, 415-445.

Grillet, M., Dominguez Gonzalez, B., Sicart, A., Pöttler, M., Cascalho, A., Billion, K., Hernandez Diaz, S., Swerts, J., Naismith, T. V., Gounko, N. V., et al. (2016). Torsins are essential regulators of cellular lipid metabolism. Dev. Cell 38, 235-247.

Grippa, A., Buxó, L., Mora, G., Funaya, C., Idrissi, F.Z., Mancuso, F., Gomez, R., Muntanyà, J., Sabidó, E., and Carvalho, P. (2015). The seipin complex Fld1/Ldb16 stabilizes ER-lipid droplet contact sites. J. Cell Biol. 211, 829-844.

Grönke, S., Beller, M., Fellert, S., Ramakrishnan, H., Jäckle, H., and Kühnlein, R.P. (2003). Control of fat storage by a Drosophila PAT Domain protein. Curr. Biol. 13, 603-606.

Grönke, S., Mildner, A., Fellert, S., Tennagels, N., Petry, S., Müller, G., Jäckle, H., and Kühnlein, R.P. (2005). Brummer lipase is an evolutionary conserved fat storage regulator in Drosophila. Cell Metab. 1, 323-330.

Grönke, S., Müller, G., Hirsch, J., Fellert, S., Andreou, A., Haase, T., Jäckle, H., and Kühnlein, R.P. (2007). Dual lipolytic control of body fat storage and mobilization in Drosophila. PLoS Biol. 5, e137.

Grönke, S., Clarke, D.-F., Broughton, S., Andrews, T.D., and Partridge, L. (2010). Molecular evolution and functional characterization of Drosophila insulin-like peptides. PLoS Genet. 6, e1000857. 
Gross, D.A., Snapp, E.L., and Silver, D.L. (2010). Structural insights into triglyceride storage mediated by fat storage-inducing transmembrane (FIT) protein 2. PLoS One 5, e10796.

Gross, D.A., Zhan, C., and Silver, D.L. (2011). Direct binding of triglyceride to fat storageinducing transmembrane proteins 1 and 2 is important for lipid droplet formation. Proc. Natl. Acad. Sci. U. S. A. 108, 19581-19586.

Groth, A.C., Fish, M., Nusse, R., and Calos, M.P. (2004). Construction of transgenic Drosophila by using the site-specific integrase from phage $\varphi C 31$. Genetics $166,1775-1782$.

Guilherme, A., Virbasius, J. V., Puri, V., and Czech, M.P. (2010). Adipocyte dysfunctions linking obesity to insulin resistance and type 2 diabetes. Nat. Rev. Mol. Cell. Biol. 9, 367-377.

Gutierrez, E., Wiggins, D., Fielding, B., and Gould, A.P. (2007). Specialized hepatocyte-like cells regulate Drosophila lipid metabolism. Nature 445, 275-280.

Haeusler, R.A., Camastra, S., Astiarraga, B., Nannipieri, M., Anselmino, M., and Ferrannini, E. (2015). Decreased expression of hepatic glucokinase in type 2 diabetes. Mol. Metab. 4, 222-226.

Hajinezhad, M.R., and Shohreh, B. (2016). Possible involvement of beta-adrenergic receptors on nociceptin / orphanin FQ induced food consumption in male rats. Zahedan J. Res. Med. Sci. $18,1-5$.

Halaas, J.L., Gajiwala, K.S., Maffei, M., Cohen, S.L., Chait, B.T., Rabinowitz, D., Lallone, R.L., Burley, S.K., and Friedman, J.M. (1995). Weight-reducing effects of the plasma protein encoded by the obese gene. Science $269,543-546$.

Hales, K.G., Korey, C.A., Larracuente, A.M., and Roberts, D.M. (2015). Genetics on the fly: a primer on the Drosophila model system. Genetics 201, 815-842.

Hall, K.D., Sacks, G., Chandramohan, D., Chow, C.C., Wang, Y.C., Gortmaker, S.L., and Swinburn, B.A. (2011). Quantification of the effect of energy imbalance on body weight. Lancet 378, 826-837.

Hall, K.D., Heymsfield, S.B., Kemnitz, J.W., Klein, S., Schoeller, D.A., and Speakman, J.R. (2012). Energy balance and its components: implications for body weight regulation. Am. J. Clin. Nutr. 95, 989-994.

Hammond, S.M., Bernstein, E., Beach, D., and Hannon, G.J. (2000). An RNA-directed nuclease mediates post-transcriptional gene silencing in Drosophila cells. Nature 404, 293 296.

Han, G.S., Wu, W.I., and Carman, G.M. (2006). The Saccharomyces cerevisiae lipin homolog is a Mg2+-dependent phosphatidate phosphatase enzyme. J. Biol. Chem. 281, 9210-9218.

Handschin, C., Chin, S., Li, P., Liu, F., Maratos-Flier, E., LeBrasseur, N.K., Yan, Z., and Spiegelman, B.M. (2007). Skeletal muscle fiber-type switching, exercise intolerance, and myopathy in PGC-1a muscle-specific knock-out animals. J. Biol. Chem. 282, 30014-30021.

Hanlon, C.D., and Andrew, D.J. (2015). Outside-in signaling - a brief review of GPCR 
signaling with a focus on the Drosophila GPCR family. J. Cell Sci. 3533-3542.

Hannon, G.J. (2002). RNA interference. Nature 418, 244-251.

Hansen, M., Flatt, T., and Aguilaniu, H. (2013). Reproduction, fat metabolism, and life span: what is the connection? Cell Metab. 17, 10-19.

Hehlert, P. (2016). PhD thesis: Function of the $\alpha$ / $\beta$-hydrolase fold family proteins Pummelig (CG1882) and hormone-sensitive lipase in the Drosophila melanogaster lipid metabolism. Georg-August University Göttingen.

Hepler, J.R., and Gilman, A.G. (1992). G proteins. Trends Biochem. Sci. 17, 383-387.

Hergarden, A.C., Tayler, T.D., and Anderson, D.J. (2012). Allatostatin-A neurons inhibit feeding behavior in adult Drosophila. Proc. Natl. Acad. Sci. 109, 3967-3972.

Hewes, R.S., and Taghert, P.H. (2001). Neuropeptides and neuropeptide receptors in the Drosophila melanogaster genome. Genome Biol. 11, 1126-1142.

Hildebrandt, A., Bickmeyer, I., and Kühnlein, R.P. (2011). Reliable Drosophila body fat quantification by a coupled colorimetric assay. PLoS One 6, e23796.

Hill, J.O. (2006). Understanding and addressing the epidemic of obesity: an energy balance perspective. Endocr. Rev. 27, 750-761.

Hill, J.O., and Commerford, R. (1996). Physical activity, fat balance, and energy balance. Int. J. Sport Nutr. 6, 80-92.

Hill, J.O., Wyatt, H.R., and Peters, J.C. (2012). Energy balance and obesity. Circulation 126, $126-132$.

Holoch, D., and Moazed, D. (2015). RNA-mediated epigenetic regulation of gene expression. Nat. Rev. Genet. 16, 71-84.

Honegger, B., Galic, M., Köhler, K., Wittwer, F., Brogiolo, W., Hafen, E., and Stocker, H. (2008). Imp-L2, a putative homolog of vertebrate IGF-binding protein 7, counteracts insulin signaling in Drosophila and is essential for starvation resistance. J. Biol. 7, 10.

Van der Horst, D.J., Van Marrewijk, W.J.A., and Diederen, J.H.B. (2001). Adipokinetic hormones of insect: release, signal transduction, and responses. Int. Rev. Cytol. 211, 179240.

Horton, T.J., Drougas, H., Brachey, A., Reed, G.W., Peters, J.C., and Hill, J.O. (1995). Fat and carbohydrate overfeeding in humans: different effects on energy storage. Am. J. Clin. Nutr. 62, 19-29.

Hotamisligil, G.S. (2017). Inflammation, metaflammation and immunometabolic disorders. Nature $542,177-185$.

Hotamisligil, G.S., Shargill, N.S., and Spiegelman, B.M. (1993). Adipose expression of tumor necrosis factor- $\alpha$ : direct role in obesity-linked insulin resistance. Science 259, 87-91. 
Hotamisligil, G.S., Arner, P., Caro, J.F., Atkinson, R.L., and Spiegelman, B.M. (1995). Increased adipose tissue expression of tumor necrosis factor- $\alpha$ in human obesity and insulin resistance. J. Clin. Invest. 95, 2409-2415.

Hotamisligil, G.S., Peraldi, P., Budavari, A., and Ellis, R. (1996). IRS-1-mediated inhibition of insulin receptor tyrosine kinase activity in TNF- $\alpha$ and obesity-induced insulin resistance. Science 271, 665-668.

Howe, L.R., Subbaramaiah, K., Hudis, C.A., and Dannenberg, A.J. (2013). Molecular pahways: adipose inflammation as a mediator of obesity-associated cancer. Clin. Cancer Res. 19, 6074-6083.

Hruby, A., and Hu, F.B. (2015). The epidemiology of obesity: a big picture. Pharmacoeconomics 33, 673-689.

Hu, F.B., Li, T.Y., Colditz, G.A., Willet, W.C., and Manson, J.E. (2003). Television watching and other sedentary behaviors in relation to risk of obesity and type 2 diabetes mellitus in women. JAMA 289, 1785-1791.

Hu, J.X., Thomas, C.E., and Brunak, S. (2016). Network biology concepts in complex disease comorbidities. Nat. Rev. Genet. 17, 615-629.

Huang, Y., Huang, S., Lam, S.M., Liu, Z., Shui, G., and Zhang, Y.Q. (2016). Acsl, the Drosophila ortholog of intellectual-disability-related ACSL4, inhibits synaptic growth by altered lipids. J Cell Sci 129, 4034-4045.

Hussain, M.A., Akalestou, E., and Song, W. (2016). Inter-organ communication and regulation of beta cell function. Diabetologia 59, 659-667.

Ida, T., Takahashi, T., Tominaga, H., Sato, T., Sano, H., Kume, K., Ozaki, M., Hiraguchi, T., Shiotani, H., Terajima, S., et al. (2012). Isolation of the bioactive peptides CCHamide-1 and CCHamide-2 from Drosophila and their putative role in appetite regulation as ligands for $G$ protein-coupled receptors. Front. Endocrinol. (Lausanne). 3, 177.

lijima, K., Zhao, L., Shenton, C., and lijima-Ando, K. (2009). Regulation of energy stores and feeding by neuronal and peripheral CREB activity in Drosophila. PLoS One 4, e8498.

Incio, J., Tam, J., Rahbari, N.N., Suboj, P., McManus, D.T., Chin, S.M., Vardam, T.D., Batista, A., Babykutty, S., Jung, K., et al. (2016). PIGF/VEGFR-1 signaling promotes macrophage polarization and accelerated tumor progression in obesity. Clin. Cancer Res. 22, 2993-3004.

Ingalls, A.M., Dickie, M.M., and Snell, G.D. (1950). Obese, a new mutation in the house mouse. J. Hered. 41, 317-318.

Ivanov, P.C., and Bartsch, R.P. (2014). Network physiology: mapping interactions between networks of physiologic networks (Springer International Publishing Switzerland).

Ivanov, P.C.H., Liu, K.K.L., and Bartsch, R.P. (2016). Focus on the emerging new fields of network physiology and network medicine. New J. Phys. 18, 100201.

Ja, W.W., Carvalho, G.B., Mak, E.M., de la Rosa, N.N., Fang, A.Y., Liong, J.C., Brummel, T., 
and Benzer, S. (2007). Prandiology of Drosophila and the CAFE assay. Proc. Natl. Acad. Sci. U. S. A. $104,8253-8256$.

Jensen, M. (2008). Role of body fat distribution and the metabolic complications of obesity. J. Clin. Endocrinol. Metab. 93, 57-63.

Jourjine, N., Mullaney, B.C., Mann, K., and Scott, K. (2016). Coupled sensing of hunger and thirst signals balances sugar and water consumption. Cell 166, 855-866.

Jumbo-Lucioni, P., Ayroles, J.F., Chambers, M.M., Jordan, K.W., Leips, J., Mackay, T.F., and De Luca, M. (2010). Systems genetics analysis of body weight and energy metabolism traits in Drosophila melanogaster. BMC Genomics 11, 297.

Jung, U., Jiang, X., Kaufmann, S.H.E., and Patzel, V. (2013). A universal TaqMan-based RTPCR protocol for cost-efficient detection of small noncoding RNA. RNA 19, 1864-1873.

Jünger, M.A., Rintelen, F., Stocker, H., Wasserman, J.D., Végh, M., Radimerski, T., Greenberg, M.E., Hafen, E., Takahashi, Y., Kadowaki, H., et al. (2003). The Drosophila Forkhead transcription factor FOXO mediates the reduction in cell number associated with reduced insulin signaling. J. Biol. 2, 20.

Kahn, B.B., and Flier, J.S. (2000). Obesity and insulin resistance. J. Clin. Invest. 106, 473481.

Kain, P., Chakraborty, T.S., Sundaram, S., Siddiqi, O., Rodrigues, V., and Hasan, G. (2008). Reduced odor responses from antennal neurons of $G(q) \alpha$, phospholipase $C \beta$, and $\operatorname{rdg} A$ mutants in Drosophila support a role for a phospholipid intermediate in insect olfactory transduction. J. Neurosci. 28, 4745-4755.

Kaluzny, M.A., Duncan, L.A., Merritt, M. V., and Epps, D.E. (1985). Rapid separation of lipid classes in high yield and purity using bonded phase columns. J. Lipid Res. 26, 135-140.

Kang, S.M., Yoon, J.W., Ahn, H.Y., Kim, S.Y., Lee, K.H., Shin, H., Choi, S.H., Park, K.S., Jang, H.C., and Lim, S. (2011). Android fat depot is more closely associated with metabolic syndrome than abdominal visceral fat in elderly people. PLoS One 6, e27694.

Kanzler, S.A., Januario, A.C., and Paschoalini, M.A. (2011). Involvement of $\beta 3$-adrenergic receptors in the control of food intake in rats. Brazilian J. Med. Biol. Res. 44, 1141-1147.

Katewa, S.D., Demontis, F., Kolipinski, M., Hubbard, A., Gill, M.S., Perrimon, N., Melov, S., and Kapahi, P. (2012). Intramyocellular fatty-acid metabolism plays a critical role in mediating responses to dietary restriction in Drosophila melanogaster. Cell Metab. 16, 97-103.

Keith, A.D. (1967). Fatty acid metabolism in Drosophila melanogaster: interaction between dietary fatty acids and de novo synthesis. Comp. Biochem. Physiol. 21, 587-600.

Kennerdell, J.R., and Carthew, R.W. (2000). Heritable gene silencing in Drosophila using double-stranded RNA. Nat. Biotechnol. 18, 896-898.

Kershaw, E.E., and Filier, J.S. (2004). Adipose Tissue as an endocrine organ. J. Clin. Endocrinol. Metab. 89, 229-237. 
Keys, A., Fidanza, F., Karvonen, M.J., Kimura, N., and Taylor, H.L. (1972). Indices of relative weight and obesity. J. Chronic Dis. 25, 329-343.

Kim, J., and Neufeld, T.P. (2015). Dietary sugar promotes systemic TOR activation in Drosophila through AHK-dependent selective secretion of Dilp3. Nat. Commun. 6, 6846.

Kim, S.K., and Rulifson, E.J. (2004). Conserved mechanisms of glucose sensing and regulation by Drosophila corpora cardiaca cells. Nature 431, 316-320.

Kirst, C., Timme, M., and Battaglia, D. (2016). Dynamic information routing in complex networks. Nat. Commun. 7, 11061 .

Klein, R.F., Troyer, W.G., Back, K.W., Hood, T.C., and Bogdonoff, M.D. (1965). Lipid mobilization in lean and obese subjects. Ann. N. Y. Acad. Sci. 131, 662-672.

Klemenz, R., Weber, U., and J.Gehring, W. (1987). The white gene as a marker in a new Pelement vector for gene transfer in Drosophila. Nucleic Acids Res. 15, 3947-3959.

Klepsatel, P., Gáliková, M., Xu, Y., and Kühnlein, R.P. (2016). Thermal stress depletes energy reserves in Drosophila. Sci. Rep. 6, 33667. doi: 10.1038/srep33667.

Kohyama-Koganeya, A., Kim, Y.J., Miura, M., and Hirabayashi, Y. (2008). A Drosophila orphan G protein-coupled receptor BOSS functions as a glucose-responding receptor: loss of boss causes abnormal energy metabolism. Proc. Natl. Acad. Sci. U. S. A. 105, 15328-15333.

Kondo, S., and Ueda, R. (2013). Highly improved gene targeting by germline-specific Cas9 expression in Drosophila. Genetics 195, 715-721.

Koyama, T., and Mirth, C.K. (2016). Growth-blocking peptides as nutrition-sensitive signals for insulin secretion and body size regulation. PLoS Biol. 14, e1002392.

Krahmer, N., Guo, Y., Wilfling, F., Hilger, M., Lingrell, S., Heger, K., Newman, H.W., SchmidtSupprian, M., Vance, D.E., Mann, M., et al. (2011). Phosphatidylcholine synthesis for lipid droplet expansion is mediated by localized activation of CTP:Phosphocholine cytidylyltransferase. Cell Metab. 14, 504-515.

Kramer, J.M., Davidge, J.T., Lockyer, J.M., and Staveley, B.E. (2003). Expression of Drosophila FOXO regulates growth and can phenocopy starvation. BMC Dev. Biol. 3, 5 .

Krashes, M.J., DasGupta, S., Vreede, A., White, B., Armstrong, J.D., and Waddell, S. (2009). A neural circuit mechanism integrating motivational state with memory expression in Drosophila. Cell 139, 416-427.

Kréneisz, O., Chen, X., Fridell, Y.-W.C., and Mulkey, D.K. (2010). Glucose increases activity and $\mathrm{Ca}^{2+}$ in insulin-producing cells of adult Drosophila. Neuroreport 21, 1116-1120.

Kubrak, O.I., Lushchak, O. V, Zandawala, M., and Na, D.R. (2016). Systemic corazonin signalling modulates stress responses and metabolism in Drosophila. Open Biol. 6, 160152.

Kühnlein, R.P. (2011). The contribution of the Drosophila model to lipid droplet research. Prog. Lipid Res. 50, 348-356. 
Kühnlein, R.P. (2012). Lipid droplet-based storage fat metabolism in Drosophila. J. Lipid Res. 53, 28-33.

Kunst, M., Hughes, M.E., Raccuglia, D., Felix, M., Li, M., Barnett, G., and Duah, J. (2014). Calcitonin gene-related peptide neurons mediate sleep-specific circadian output in Drosophila. Curr. Biol. 24, 2652-2664.

Kwon, Y., Song, W., Asara, J.M., Perrimon, N., Kwon, Y., Song, W., Droujinine, I.A., Hu, Y., Asara, J.M., and Perrimon, N. (2015). Systemic organ wasting induced by localized expression of the secreted insulin / IGF antagonist ImpL2. Dev. Cell 33, 36-46.

Lacruz, R.S., and Feske, S. (2015). Diseases caused by mutations in ORAl1 and STIM1. Ann. N. Y. Acad. Sci. 1356, 45-79.

Langin, D., Dicker, A., Tavernier, G., Hoffstedt, J., Mairal, A., Rydén, M., Arner, E., Sicard, A., Jenkins, C.M., Viguerie, N., et al. (2005). Adipocyte lipases and defect of lipolysis in human obesity. Diabetes 54, 3190-3197.

Laplante, M., and Sabatini, D.M. (2009). An emerging role of mTOR in lipid biosynthesis. Curr. Biol. 19, R1046-R1052.

Lasorsa, F.M., Pinton, P., Palmieri, L., Fiermonte, G., Rizzuto, R., and Palmieri, F. (2003). Recombinant expression of the $\mathrm{Ca}^{2+}$-sensitive aspartate/glutamate carrier increases mitochondrial ATP production in agonist-stimulated chinese hamster ovary cells. J. Biol. Chem. 278, 38686-38692.

Lass, A., Zimmermann, R., Oberer, M., and Zechner, R. (2011). Lipolysis - a highly regulated multi-enzyme complex mediates the catabolism of cellular fat stores. Prog. Lipid Res. 50, 14 27.

Leclerc, I., Sun, G., Morris, C., Fernandez-Millan, E., Nyirenda, M., and Rutter, G.A. (2011). AMP-activated protein kinase regulates glucagon secretion from mouse pancreatic alpha cells. Diabetologia 54, 125-134.

Lee, G., and Park, J.H. (2004). Hemolymph sugar homeostasis and starvation-induced hyperactivity affected by genetic manipulations of the adipokinetic hormone-encoding gene in Drosophila melanogaster. Genetics 167, 311-323.

Lee, Y., and Montell, C. (2013). Drosophila TRPA1 functions in temperature control of circadian rhythm in pacemaker neurons. J Neurosci. 33, 6716-6725.

Lee, K.-S., Kwon, O.-Y., Lee, J.H., Kwon, K., Min, K.-J., Jung, S.-A., Kim, A.-K., You, K.-H., Tatar, M., and Yu, K. (2008). Drosophila short neuropeptide F signalling regulates growth by ERK-mediated insulin signalling. Nat. Cell Biol. 10, 468-475.

Lee, K.S., You, K.H., Choo, J.K., Han, Y.M., and Yu, K. (2004). Drosophila short neuropeptide $F$ regulates food intake and body size. J. Biol. Chem. 279, 50781-50789.

Lee, S., Bao, H., Ishikawa, Z., Wang, W., Lim, H.-Y., Barsh, G., Farooqi, I., O'Rahilly, S., Kopelman, P., Luchsinger, J., et al. (2017). Cardiomyocyte regulation of systemic lipid metabolism by the apolipoprotein B-containing lipoproteins in Drosophila. PLOS Genet. 13, e1006555. 
Leevers, S.J., Weinkove, D., MacDougall, L.K., Hafen, E., and Waterfield, M.D. (1996). The Drosophila phosphoinositide 3-kinase Dp110 promotes cell growth. EMBO J. 15, 6584-6594.

Levian, C., Ruiz, E., and Yang, X. (2014). The pathogenesis of obesity from a genomic and systems biology perspective. Yale J. Biol. Med. 87, 113-126.

Levin, L.R., Han, P.L., Hwang, P.M., Feinstein, P.G., Davis, R.L., and Reed, R.R. (1992). The Drosophila learning and memory gene rutabaga encodes a $\mathrm{Ca}^{2+}$ calmodulin-responsive adenylyl cyclase. Cell 68, 479-489.

Li, Y., Hoffmann, J., Li, Y., Stephano, F., Bruchhaus, I., Fink, C., and Roeder, T. (2016). Octopamine controls starvation resistance, life span and metabolic traits in Drosophila. Sci. Rep. 6, 35359.

Lim, S., and Meigs, J.B. (2013). Ectopic fat and cardiometabolic and vascular risk. Int. J. Cardiol. 169, 166-167.

Lim, H.Y., Wang, W., Wessells, R.J., Ocorr, K., and Bodmer, R. (2011). Phospholipid homeostasis regulates lipid metabolism and cardiac function through SREBP signaling in Drosophila. Genes Dev. 25, 189-200.

Linn, T.C., and Srere, P.A. (1980). Coenzyme A requirement for the termination reaction of rat liver fatty acid synthetase. J. Biol. Chem. 255, 10676-10680.

Liou, J., Kim, M.L., Won, D.H., Jones, J.T., Myers, J.W., Ferrell, J.E., and Meyer, T. (2005). STIM is a $\mathrm{Ca}^{2+}$ sensor essential for $\mathrm{Ca}^{2+}$-store- depletion-triggered $\mathrm{Ca}^{2+}$ influx. Curr. Biol. 15, 1235-1241.

Lipardi, C., and Paterson, B.M. (2009). Identification of an RNA-dependent RNA polymerase in Drosophila involved in RNAi and transposon suppression. Proc. Natl. Acad. Sci. U. S. A. 106, 15645-15650.

Lipardi, C., and Paterson, B.M. (2010). Identification of an RNA-dependent RNA polymerase in Drosophila establishes a common theme in RNA silencing. Fly (Austin). 4, 30-35.

Liu, J., Li, T., Yang, D., Ma, R., Moran, T.H., and Smith, W.W. (2012). Synphilin-1 alters metabolic homeostasis in a novel Drosophila obesity model. Int. J. Obes. 1529-1536.

Liu, L., Zhang, K., Sandoval, H., Yamamoto, S., Jaiswal, M., Sanz, E., Li, Z., Hui, J., Graham, B.H., Quintana, A., et al. (2015). Glial lipid droplets and ROS induced by mitochondrial defects promote neurodegeneration. Cell 160, 177-190.

Liu, S., Alexander, R.K., and Lee, C.H. (2014a). Lipid metabolites as metabolic messengers in inter-organ communication. Trends Endocrinol. Metab. 25, 356-363.

Liu, Z., Wang, X., Yu, Y., Li, X., Wang, T., Jiang, H., Ren, Q., Jiao, Y., Sawa, A., Moran, T., et al. (2008). A Drosophila model for LRRK2-linked parkinsonism. Proc. Natl. Acad. Sci. U. S. A. 105, 2693-2698.

Liu, Z., Huang, Y., Hu, W., Huang, S., Wang, Q., Han, J., and Zhang, Y.Q. (2014b). dAcsl, the Drosophila ortholog of acyl-CoA synthetase long-chain family member 3 and 4 , inhibits 
synapse growth by attenuating bone morphogenetic protein signaling via endocytic recycling. J. Neurosci. 34, 2785-2796.

Livak, K.J., and Schmittgen, T.D. (2001). Analysis of relative gene expression data using realtime quantitative PCR and the 2(-Delta Delta C(T)) Method. Methods 25, 402-408.

Livingstone, M.B.E., Robson, P.J., Wallace, J.M.W., and Mckinley, M.C. (2003). How active are we? Levels of routine physical activity in children and adults. Proc. Nutr. Soc. 62, 681701.

Loh, K., Herzog, H., and Shi, Y.-C. (2015). Regulation of energy homeostasis by the NPY system. Trends Endocrinol. Metab. 26, 125-135.

Louie, S.M., Roberts, L.S., and Nomura, D.K. (2013). Mechanisms linking obesity and cancer. Biochim. Biophys. Acta - Mol. Cell Biol. Lipids 1831, 1499-1508.

Love, M.I., Huber, W., and Anders, S. (2014). Moderated estimation of fold change and dispersion for RNA-seq data with DESeq2. Genome Biol. 15, 550.

Lum, P.Y., and Chino, H. (1990). Primary role of adipokinetic hormone in the formation of low density lipophorin in locusts. J. Lipid Res. 31, 2039-2044.

Luquet, S. (2005). NPY/AgRP neurons are essential for feeding in adult mice but can be ablated in neonates. Science 310, 683-685.

Lyne, R., Smith, R., Rutherford, K., Wakeling, M., Varley, A., Guillier, F., Janssens, H., Ji, W., Mclaren, P., North, P., et al. (2007). FlyMine: an integrated database for Drosophila and Anopheles genomics. Genome Biol. 8, R129.

Maida, Y., Yasukawa, M., Furuuchi, M., Lassmann, T., Possemato, R., Okamoto, N., Kasim, V., Hayashizaki, Y., Hahn, W.C., and Masutomi, K. (2009). An RNA-dependent RNA polymerase formed by TERT and the RMRP RNA. Nature 461, 230-235.

Maniar, J.M., and Fire, A.Z. (2011). EGO-1, a C. elegans RdRP, modulates gene expression via production of mRNA-templated short antisense RNAs. Curr. Biol. 21, 449-459.

Marioni, J.C., Mason, C.E., Mane, S.M., Stephens, M., and Gilad, Y. (2008). Comparison with gene expression arrays RNA-seq : an assessment of technical reproducibility and comparison with gene expression arrays. Genome Res. 18, 1509-1517.

Markow, T.A. (2015). The secret lives of Drosophila flies. Elife 4, e06793.

Van Marrewijk, W.J.A., Van Den Broek, A.T.M., Gielbert, M.L., and Van Der Horst, D.J. (1996). Insect adipokinetic hormone stimulates inositol phosphate metabolism: roles for both Ins(1,4,5)P3 and Ins(1,3,4,5)P4 in signal transduction? Mol. Cell. Endocrinol. 122, 141-150.

Maryam, R., Bianca, K., Michael, M., and Sander, K. (2010). Peroxisome proliferatoractivated receptor alpha target genes. PPAR Research. 2010, Article ID 612089.

Mascie-Taylor, C.G.N., and Goto, R. (2007). Human variation and body mass index: a review of the universality of BMI cut-offs, gender and urban-rural differences, and secular changes. J. Physiol. Anthropol. 26, 109-112. 
Massa, M.L., Gagliardino, J.J., and Francini, F. (2011). Liver glucokinase: an overview on the regulatorymechanisms of its activity. IUBMB Life $63,1-6$.

Masuyama, K., Zhang, Y., Rao, Y., and Wang, J.W. (2012). Mapping neural circuits with activity-dependent nuclear import of a transcription factor. J. Neurogenet. 26, 89-102.

Matsuda, H., Yamada, T., Yoshida, M., and Nishimura, T. (2015). Flies without trehalose. J. Biol. Chem. 290, 1244-1255.

Mattila, J., Havula, E., Suominen, E., Teesalu, M., Surakka, I., Hynynen, R., Kilpinen, H., Väänänen, J., Hovatta, I., Käkelä, R., et al. (2015). Mondo-Mlx mediates organismal sugar sensing through the Gli-similar transcription factor sugarbabe. Cell Rep. 13, 350-364.

Maus, M., Cuk, M., Patel, B., Lian, J., Ouimet, M., Kaufmann, U., Yang, J., Horvath, R., Hornig-Do, H.-T., Chrzanowska-Lightowlers, Z.M., et al. (2017). Store-Operated Ca ${ }^{2+}$ Entry controls induction of lipolysis and the transcriptional reprogramming to lipid metabolism. Cell Metab. 25, 698-712.

McGarry, J.D. (1992). What If Minkowski had been ageusic? an alternative angle on diabetes. Science 258, 766-770.

McGuire, S.E. (2003). Spatiotemporal rescue of memory dysfunction in Drosophila. Science 302, 1765-1768.

Mello, C.C., and Conte, D. (2004). Revealing the world of RNA interference. Nature 431, 338-342.

Min, S., Chae, H.S., Jang, Y.H., Choi, S., Lee, S., Jeong, Y.T., Jones, W.D., Moon, S.J., Kim, Y.J., and Chung, J. (2016). Identification of a peptidergic pathway critical to satiety responses in Drosophila. Curr. Biol. 26, 814-820.

Miyamoto, T., Slone, J., Song, X., and Amrein, H. (2012). A fructose receptor functions as a nutrient sensor in the Drosophila brain. Cell 151, 1113-1125.

Moessinger, C., Klizaite, K., Steinhagen, A., Philippou-Massier, J., Shevchenko, A., Hoch, M., Ejsing, C.S., and Thiele, C. (2014). Two different pathways of phosphatidylcholine synthesis, the Kennedy Pathway and the Lands Cycle, differentially regulate cellular triacylglycerol storage. BMC Cell Biol. 15, 43.

Montagne, J., Stewart, M.J., Stocker, H., Hafen, E., Kozma, S.C., and Thomas, G. (1999). Drosophila S6 kinase: a regulator of cell size. Science 285, 2126-2129.

Montague, C.T., Farooqi, I.S., Whitehead, J.P., Soos, M. a, Rau, H., Wareham, N.J., Sewter, C.P., Digby, J.E., Mohammed, S.N., Hurst, J.A., et al. (1997). Congenital leptin deficiency is associated with severe early-onset obesity in humans. Nature 387, 903-908.

Montgomery, M.K., Xu, S., and Fire, A (1998). RNA as a target of double-stranded RNAmediated genetic interference in Caenorhabditis elegans. Proc. Natl. Acad. Sci. U. S. A. 95, 15502-15507.

Te Morenga, L., Mallard, S., Mann, J., and Morenga, L. Te (2013). Dietary sugars and body 
weight: systematic review and meta-analyses of randomised controlled trials and cohort studies. BMJ. 346, e7492.

Moreno-Viedma, V., Amor, M., Sarabi, A., Bilban, M., Staffler, G., Zeyda, M., and Stulnig, T.M. (2016). Common dysregulated pathways in obese adipose tissue and atherosclerosis. Cardiovasc. Diabetol. 15, 120.

Muller, H.J. (1918). Genetic variability, twin hybrids and constant hybrids, in a case of balanced lethal factors. Genetics 3, 422-499.

Müller-Riemenschneider, F., Reinhold, T., Berghöfer, A., and Willich, S.N. (2008). Healtheconomic burden of obesity in Europe. Eur. J. Epidemiol. 23, 499-509.

Musselman, L.P., Fink, J.L., Narzinski, K., Ramachandran, P.V., Hathiramani, S.S., Cagan, R.L., and Baranski, T.J. (2011). A high-sugar diet produces obesity and insulin resistance in wild-type Drosophila. Dis. Model. Mech. 4, 842-849.

Nakazato, M., Murakami, N., Date, Y., Kojima, M., Matsuo, H., Kangawa, K., and Matsukura, S. (2001). A role for ghrelin in the central regulation of feeding. Nature 409, 194-198.

Nässel, D.R., Passier, P.C.C.M., Elekes, K., Dircksen, H., Vullings, H.G.B., and Cantera, R. (1995). Evidence that locustatachykinin I is involved in release of adipokinetic hormone from locust corpora cardiaca. Regul. Pept. 57, 297-310.

Nässel, D.R., Vullings, H.G., Passier, P.C., Lundquist, C.T., Schoofs, L., Diederen, J.H., and Van der Horst, D.J. (1999). Several isoforms of locustatachykinins may be involved in cyclic AMP-mediated release of adipokinetic hormones from the locust Corpora cardiaca. Gen. Comp. Endocrinol. 113, 401-412.

Neeland, I.J., Turer, A.T., Ayers, C.R., Berry, J.D., Rohatgi, A., Das, S.R., Khera, A., Vega, G.L., McGuire, D.K., Grundy, S.M., et al. (2015). Body fat distribution and incident cardiovascular disease in obese adults. J. Am. Coll. Cardiol. 65, 2150-2151.

Ng, M., Fleming, T., Robinson, M., Thomson, B., Graetz, N., Margono, C., Mullany, E.C., Biryukov, S., Abbafati, C., Abera, S.F., et al. (2014). Global, regional, and national prevalence of overweight and obesity in children and adults during 1980-2013: a systematic analysis for the Global Burden of Disease Study 2013. Lancet 384, 766-781.

Ni, J., Markstein, M., Binari, R., Pfeiffer, B., Liu, L., Villalta, C., Booker, M., Perkins, L., and Perrimon, N. (2008). Vector and parameters for targeted transgenic RNA interference in Drosophila melanogaster. Nat. Methods 5, 49-51.

Nichols, R., Schneuwly, S.A., and Dixon, J.E. (1988). Identification and characterization of a Drosophila homologue to the vertebrate neuropeptide cholecystokinin. J. Biol. Chem. 263, 12167-12170.

Nicholson, L., Singh, G.K., Osterwalder, T., Roman, G.W., Davis, R.L., and Keshishian, H. (2008). Spatial and temporal control of gene expression in Drosophila using the inducible geneSwitch GAL4 system. I. Screen for larval nervous system drivers. Genetics 178, $215-$ 234.

Niemeyer, B.A. (2016). Changing calcium: CRAC channel (Orai and STIM) expression, 
splicing and posttranslational modifiers. Am. J. Physiol. - Cell Physiol. 310, C701-C709.

Nikolić, N., Rhedin, M., Rustan, A.C., Storlien, L., Thoresen, G.H., and Strömstedt, M. (2012). Overexpression of PGC-1 $\alpha$ increases fatty acid oxidative capacity of human skeletal muscle cells. Biochem. Res. Int. 2012, 714074.

Niwa, R., and Niwa, Y.S. (2011). The fruit fly Drosophila melanogaster as a model system to study cholesterol metabolism and homeostasis. Cholesterol 2011, 176802.

Oh, E.C., Vasanth, S., and Katsanis, N. (2015). Metabolic regulation and energy homeostasis through the primary cilium. Cell Metab. 21, 21-31.

Oldham, S., Montagne, J., Radimerski, T., Thomas, G., and Hafen, E. (2000). Genetic and biochemical characterization of dTOR , the Drosophila homolog of the target of rapamycin. Genes Dev. 14, 2689-2694.

Orlicky, D.J., Monks, J., Stefanski, A.L., and McManaman, J.L. (2013). Dynamics and molecular determinants of cytoplasmic lipid droplet clustering and dispersion. PLoS One 8. e66837.

Osterwalder, T., Yoon, K.S., White, B.H., and Keshishian, H. (2001). A conditional tissuespecific transgene expression system using inducible GAL4. Proc. Natl. Acad. Sci. U. S. A. 98, 12596-12601.

Ottaviani, E., Malagoli, D., and Franceschi, C. (2011). The evolution of the adipose tissue: a neglected enigma. Gen. Comp. Endocrinol. 174, 1-4.

Padmanabha, D., and Baker, K.D. (2014). Drosophila gains traction as a repurposed tool to investigate metabolism. Trends Endocrinol. Metab. 25, 518-527.

Palm, W., Sampaio, J.L., Brankatschk, M., Carvalho, M., Mahmoud, A., Shevchenko, A., and Eaton, S. (2012). Lipoproteins in Drosophila melanogaster-assembly, function, and influence on tissue lipid composition. PLoS Genet. 8. e1002828.

Palmieri, F., Pardo, B., Lasorsa, F.M., Arco, A. Del, Kobayashi, K., lijima, M., Satrustegui, J., Palmieri, F., Runswick, M.J., Waker, J.E., et al. (2001). Citrin and aralar1 are $\mathrm{Ca}^{2+}$-stimulated aspartate/glutamate transporters in mitochondria. EMBO J. 20,5060-5069.

Palu, R.A.S., and Thummel, C.S. (2016). Sir2 acts through hepatocyte nuclear factor 4 to maintain insulin signaling and metabolic homeostasis in Drosophila. PLoS Genet. 12, e1005978.

Pan, D.A., and Hardie, D.G. (2002). A homologue of AMP-activated protein kinase in Drosophila melanogaster is sensitive to AMP and is activated by ATP depletion. Biochem. J. 367, 179-186.

Parisi, M., Li, R., and Oliver, B. (2011). Lipid profiles of female and male Drosophila. BMC Res. Notes 4, 198.

Park, S., Alfa, R.W., Topper, S.M., Kim, G.E.S., Kockel, L., and Kim, S.K. (2014a). A genetic strategy to measure circulating Drosophila insulin reveals genes regulating insulin production and secretion. PLoS Genet. 10, e1004555. 
Park, S., Sonn, J.Y., Oh, Y., Lim, C., and Choe, J. (2014b). SIFamide and SIFamide receptor defines a novel neuropeptide signaling to promote sleep in Drosophila. Mol. Cells 37, 295301.

Park, Y., Kim, Y.-J.J., and Adams, M.E. (2002). Identification of G protein-coupled receptors for Drosophila PRXamide peptides, CCAP, corazonin, and AKH supports a theory of ligandreceptor coevolution. Proc. Natl. Acad. Sci. U. S. A. 99, 11423-11428.

Patel, P., and Abate, N. (2013). Body fat distribution and insulin resistance. Nutrients 5, 2019-2027.

Patel, R.T., Soulages, J.L., Hariharasundaram, B., and Arrese, E.L. (2005). Activation of the lipid droplet controls the rate of lipolysis of triglycerides in the insect fat body. J. Biol. Chem. 280, 22624-22631.

Pathak, T., Trivedi, D., and Hasan, G. (2017). CRISPR-Cas induced mutants identify a requirement for dSTIM in larval dopaminergic cells of Drosophila melanogaster. G3 Genes|Genomes|Genetics 7, 923-933.

Perkins, L.A., Holderbaum, L., Tao, R., Hu, Y., Sopko, R., McCall, K., Yang-Zhou, D., Flockhart, I., Binari, R., Shim, H.S., et al. (2015). The transgenic RNAi project at Harvard medical school: resources and validation. Genetics 201, 843-852.

Perrimon, N., Ni, J., Perkins, L., Noller, H.F., Volpe, T., and Martienssen, R.A. (2012). In vivo RNAi : today and tomorrow. Cold Spring Harb Perspect. Biol. 2, a003640.

Petsakou, A., and Perrimon, N. (2016). "ISN't Thirst Sweet?" Says the Fly. Cell 166, 796-797.

Petursson, H., Sigurdsson, J.A., Bengtsson, C., Nilsen, T.I.L., and Getz, L. (2011). Body configuration as a predictor of mortality: comparison of five anthropometric measures in a 12 year follow-up of the norwegian HUNT 2 study. PLoS One 6. e26621.

Pietrocola, F., Galluzzi, L., Bravo-San Pedro, J.M., Madeo, F., and Kroemer, G. (2015). Acetyl coenzyme A: a central metabolite and second messenger. Cell Metab. 21, 805-821.

Pischon, T., Boeing, H., Hoffmann, K., Bergmann, M., Schulze, M.B., Overvad, K., van der Schouw, Y.T., Spencer, E., Moons, K.G.M., Tjønneland, A., et al. (2008). General and abdominal adiposity and risk of death in Europe. N. Engl. J. Med. 359, 2105-2120.

Pitsouli, C., and Perrimon, N. (2008). Developmental biology: our fly cousins' gut. Nature 454, 592-593.

Poirier, L., Shane, A., Zheng, J., and Seroude, L. (2008). Characterization of the Drosophila Gene-Switch system in aging studies: a cautionary tale. Aging Cell 7, 758-770.

Pol, A., Gross, S.P., and Parton, R.G. (2014). Biogenesis of the multifunctional lipid droplet: lipids, proteins, and sites. J. Cell Biol. 204, 635-646.

Ponton, F., Chapuis, M.P., Pernice, M., Sword, G.A., and Simpson, S.J. (2011). Evaluation of potential reference genes for reverse transcription-qPCR studies of physiological responses in Drosophila melanogaster. J. Insect Physiol. 57, 840-850. 
Pool, A.H., and Scott, K. (2014). Feeding regulation in Drosophila. Curr. Opin. Neurobiol. 29, 57-63.

Popkin, B.M., Kim, S., Rusev, E.R., Du, S., and Zizza, C. (2006). Measuring the full Economic costs of diet, physical activity and obesity-related chronic diseases. J. Int. Assoc. Study Obes. 7, 271-293.

Pories, W.J., Dohm, L.G., and Mansfield, C.J. (2010). Beyond the BMI: the search for better guidelines for bariatric surgery. Obesity (Silver Spring). 18, 865-871.

Pospisilik, J.A., Schramek, D., Schnidar, H., Cronin, S.J.F., Nehme, N.T., Zhang, X., Knauf, C., Cani, P.D., Aumayr, K., Todoric, J., et al. (2010). Drosophila genome-wide obesity screen reveals hedgehog as a determinant of brown versus white adipose cell fate. Cell 140, 148160.

Puig, O., Marr, M.T.M., Ruhf, M.L., and Tjian, R. (2003). Control of cell number by Drosophila FOXO : downstream and feedback regulation of the insulin receptor pathway. Genes Dev. 17, 2006-2020.

Racotta, R., and Soto-Mora, L.M. (1993). Specificity of alpha- and beta-adrenergic inhibition of water and food intake. Physiol. Behav. 53, 361-365.

Raffaello, A., Mammucari, C., Gherardi, G., and Rizzuto, R. (2016). Calcium at the center of cell signaling: interplay between endoplasmic reticulum, mitochondria, and lysosomes. Trends Biochem. Sci. 41, 1035-1049.

Rajan, A., and Perrimon, N. (2012). Drosophila cytokine unpaired 2 regulates physiological homeostasis by remotely controlling insulin secretion. Cell 151, 123-137.

Rajan, A., and Perrimon, N. (2013). Of flies and men: insights on organismal metabolism from fruit flies. BMC Biol. 11, 38 .

Ravussin, E., and Swinburn, B.A. (1992). Pathophysiology of obesity. Lancet 340, 404-408.

Rees, D.C., Johnson, E., and Lewinson, O. (2009). ABC transporters: the power to change. Nat. Rev. Mol. Cell Biol. 10, 218-227.

Reiff, T., Jacobson, J., Cognigni, P., Antonello, Z., Ballesta, E., Tan, K.J., Yew, J.Y., Dominguez, M., and Miguel-Aliaga, I. (2015). Endocrine remodelling of the adult intestine sustains reproduction in Drosophila. Elife 4, e06930.

Reis, T., van Gilst, M.R., and Hariharan, I.K. (2010). A buoyancy-based screen of Drosophila larvae for fat-storage mutants reveals a role for Sir2 in coupling fat Storage to nutrient availability. PLoS Genet. 6. e1001206.

Reiter, L.T., Potocki, L., Chien, S., Gribskov, M., and Bier, E. (2001). A systematic analysis of human disease-associated gene sequences in Drosophila melanogaster. Genome Res. 11, $1114-1125$.

Ren, G.R., Hauser, F., Rewitz, K.F., Kondo, S., Engelbrecht, A.F., Didriksen, A.K., Schjøtt, S.R., Sembach, F.E., Li, S., Søgaard, K.C., et al. (2015). CCHamide-2 is an orexigenic brain- 
gut peptide in Drosophila. PLoS One 10, e0133017.

Renobales, M. De, and Blomquist, G.J. (1994). Biosynthesis of medium chain fatty acids in Drosophila. Arch. Biochem. Biophys. 228, 407-414.

Reyes-DelaTorre, A., Pena-Rangel, M.T., and Riesgo-Escovar, J.R. (2017). Carbohydrate metabolism in Drosophila: reliance on the disaccharide trehalose in Carbohydrates Comprehensive Studies on Glycobiology and Glycotechnology, (InTech).

Rieder, L.E., and Larschan, E.N. (2014). Wisdom from the fly. Trends Genet. 30, 479-481.

Ritter, S.L., and Hall, R.A. (2009). Fine-tuning of GPCR activity by receptor-interacting proteins. Nat. Rev. Mol. Cell Biol. 10, 819-830.

Rizzuto, R., De Stefani, D., Raffaello, A., and Mammucari, C. (2012). Mitochondria as sensors and regulators of calcium signalling. Nat. Rev. Mol. Cell Biol. 13, 566-578.

Roda, C., Charreire, H., Feuillet, T., Mackenbach, J.D., Compernolle, S., Glonti, K., Bárdos, H., Rutter, H., McKee, M., Brug, J., et al. (2016). Lifestyle correlates of overweight in adults: a hierarchical approach (the SPOTLIGHT project). Int. J. Behav. Nutr. Phys. Act. 13, 114.

Rodgers, J.T., Lerin, C., Haas, W., Gygi, S.P., Spiegelman, B.M., and Puigserver, P. (2005). Nutrient control of glucose homeostasis through a complex of PGC-1alpha and SIRT1. Nature 434, 113-118.

Rouillard A.D., Gundersen G.W., Fernandez N.F., Wang Z., Monteiro C.D., McDermott M.G., Ma'ayan A. (2016). The harmonizonme: a collection of processed datasets gathered to serve and mine knowledge about genes and proteins. Database (Oxford) 2016, pii: baw100.

Van Roessel, P., Hayward, N.M., Barros, C.S., and Brand, A.H. (2002). Two-color GFP imaging demonstrates cell-autonomy of GAL4-driven RNA interference in Drosophila. Genesis 34, 170-173.

Roignant, J.-Y., Carré, C., Mugat, B., Szymczak, D., Lepesant, J.-A., and Antoniewski, C. (2003). Absence of transitive and systemic pathways allows cell-specific and isoform-specific RNAi in Drosophila. RNA 9, 299-308.

Roman, G., Endo, K., Zong, L., and Davis, R.L. (2001). P[Switch], a system for spatial and temporal control of gene expression in Drosophila melanogaster. Proc. Natl. Acad. Sci. U. S. A. $98,12602-12607$.

Romero-Corral, A., Somers, V.K., Sierra-Johnson, J., Thomas, R.J., Collazo-Clavell, M.L., Korinek, J., Allison, T.G., Batsis, J.A., Sert-Kuniyoshi, F.H., and Lopez-Jimenez, F. (2008). Accuracy of body mass index in diagnosing obesity in the adult general population. Int. J. Obes. (Lond.). 32, 959-966.

Roos, J., DiGregorio, P.J., Yeromin, A. V., Ohlsen, K., Lioudyno, M., Zhang, S., Safrina, O., Kozak, J.A., Wagner, S.L., Cahalan, M.D., et al. (2005). STIM1, an essential and conserved component of store-operated Ca 2+ channel function. J. Cell Biol. 169, 435-445.

Rosenheck, R. (2008). Fast food consumption and increased caloric intake: a systematic review of a trajectory towards weight gain and obesity risk. Obes. Rev. 9, 535-547. 
R Core Team (2016). R: A language and environment for statistical computing. (Version 3.3.1). R Foundation for Statistical Computing, Vienna, Austria. URL: https://www.Rproject.org/.

RStudio (2015). RStudio: Integrated Development for R. (Version 0.99.903). Boston, U.S.A. URL: http://www.rstudio.org/.

Rubin, G.M., and Spradling, A.C. (1982). Genetic transformation of Drosophila with transposable element vectors. Science 218, 348-353.

Rubin, G.M., Hong, L., Brokstein, P., Evans-Holm, M., Frise, E., Stapleton, M., and Harvey, D.A. (2000). A Drosophila complementary DNA resource. Science 287, 2222-2224.

Ruiz, A., Serrano, R., and Ariño, J. (2008). Direct regulation of genes involved in glucose utilization by the calcium/calcineurin pathway. J. Biol. Chem. 283, 13923-13933.

Rulifson, E.J., Kim, S.K., and Nusse, R. (2002). Ablation of insulin-producing neurons in flies: growth and diabetic phenotypes. Science 296, 1118-1120.

Saheki, T., and Kobayashi, K. (2002). Mitochondrial aspartate glutamate carrier (citrin) deficiency as the cause of adult-onset type II citrullinemia (CTLN2) and idiopathic neonatal hepatitis (NICCD). J. Hum. Genet. 47, 333-341.

Salo, V.T., Belevich, I., Li, S., Karhinen, L., Vihinen, H., Vigouroux, C., Magré, J., Thiele, C., Hölttä-Vuori, M., Jokitalo, E., et al. (2016). Seipin regulates ER-lipid droplet contacts and cargo delivery. EMBO J. 35, 2699-2716.

Sanders, F.W.B., and Griffin, J.L. (2016). De novo lipogenesis in the liver in health and disease: more than just a shunting yard for glucose. Biol. Rev. 91, 452-468.

Sano, H., Nakamura, A., Texada, M.J., Truman, J.W., Ishimoto, H., Kamikouchi, A., Nibu, Y., Kume, K., Ida, T., and Kojima, M. (2015). The nutrient-responsive hormone CCHamide-2 controls growth by regulating insulin-like peptides in the brain of Drosophila melanogaster. PLoS Genet. 11, e1005209.

Savage, D.B., Petersen, K.F., and Shulman, G.I. (2007). Disordered lipid metabolism and the pathogenesis of insulin resistance. Physiol. Rev. 87, 507-520.

Sayeed, O., and Benzer, S. (1996). Behavioral genetics of thermosensation and hygrosensation in Drosophila. Proc. Natl. Acad. Sci. U. S. A. 93, 6079-6084.

Scanga, S.E., Ruel, L., Binari, R.C., Snow, B., Stambolic, V., Bouchard, D., Peters, M., Calvieri, B., Mak, T.W., Woodgett, J.R., et al. (2000). The conserved PI3'K/PTEN/Akt signaling pathway regulates both cell size and survival in Drosophila. Oncogene 19, 39713977.

Schlegel, A., and Stainier, D.Y.R. (2007). Lessons from "lower" organisms: what worms, flies, and zebrafish can teach us about human energy metabolism. PLoS Genet. 3, e199.

Schleich, S., Strassburger, K., Janiesch, P.C., Koledachkina, T., Miller, K.K., Haneke, K., Cheng, Y.-S., Küchler, K., Stoecklin, G., Duncan, K.E., et al. (2014). DENR-MCT-1 promotes 
translation re-initiation downstream of uORFs to control tissue growth. Nature 512, 208-212.

Schmidt, P.T., Tornøe, K., Poulsen, S.S., Rasmussen, T.N., and Holst, J.J. (2000). Tachykinins in the porcine pancreas: potent exocrine and endocrine effects via NK-1 receptors. Pancreas 20, 241-247.

Schmittgen, T.D., and Livak, K.J. (2008). Analyzing real-time PCR data by the comparative CT method. Nat. Protoc. 3, 1101-1108.

Schoeller, D.A. (2008). Insights into energy balance from doubly labeled water. Int. J. Obes. (Lond). 32, S72-S75.

Seidell, J.C. (2010). Waist circumference and waist/hip ratio in relation to all-cause mortality, cancer and sleep apnea. Eur. J. Clin. Nutr. 64, 35-41.

Settembre, C., and Ballabio, A. (2014). Lysosome: regulator of lipid degradation pathways. Trends Cell Biol. 24, 743-750.

Shankar, S., Chua, J.Y., Tan, K.J., Calvert, M.E.K., Weng, R., Ng, W.C., Mori, K., and Yew, J.Y. (2015). The neuropeptide tachykinin is essential for pheromone detection in a gustatory neural circuit. Elife 4, e06914.

Shcherbata, H.R., Yatsenko, A.S., Patterson, L., Sood, V.D., Nudel, U., Yaffe, D., Baker, D., and Ruohola-Baker, H. (2007). Dissecting muscle and neuronal disorders in a Drosophila model of muscular dystrophy. EMBO J. 26, 481-493.

Shungin, D., Winkler, T.W., Croteau-Chonka, D.C., Ferreira, T., Locke, A.E., Magi, R., Strawbridge, R.J., Pers, T.H., Fischer, K., Justice, A.E., et al. (2015). New genetic loci link adipose and insulin biology to body fat distribution. Nature 518, 187-196.

Sieber, M.H., and Thummel, C.S. (2009). The DHR96 nuclear receptor controls triacylglycerol homeostasis in Drosophila. Cell Metab. 10, 481-490.

Simpson, J. a, MacInnis, R.J., Peeters, A., Hopper, J.L., Giles, G.G., and English, D.R. (2007). A comparison of adiposity measures as predictors of all-cause mortality: the Melbourne Collaborative Cohort Study. Obesity (Silver Spring). 15, 994-1003.

Singh, R., Kaushik, S., Wang, Y., Xiang, Y., Novak, I., Komatsu, M., Tanaka, K., Cuervo, A.M., and Czaja, M.J. (2009). Autophagy regulates lipid metabolism. Nature 458, 1131-1135.

Skorupa, D.A., Dervisefendic, A., Zwiener, J., and Pletcher, S.D. (2008). Dietary composition specifies consumption, obesity, and lifespan in Drosophila melanogaster. Aging Cell 7, 478490.

Slack, C., Giannakou, M.E., Foley, A., Goss, M., and Partridge, L. (2011). dFOXOindependent effects of reduced insulin-like signaling in Drosophila. Aging Cell 10, 735-748.

Smemo, S., Tena, J.J., Kim, K.-H., Gamazon, E.R., Sakabe, N.J., Gómez-Marín, C., Aneas, I., Credidio, F.L., Sobreira, D.R., Wasserman, N.F., et al. (2014). Obesity-associated variants within FTO form long-range functional connections with IRX3. Nature 507, 371-375.

Smith, U. (2015). Abdominal obesity: a marker of ectopic fat accumulation. J. Clin. Investig. 
Smith, W.W., Thomas, J., Liu, J., Li, T., and Moran, T.H. (2014). From fat fruit fly to human obesity. Physiol. Behav. 136, 15-21.

Soboloff, J., Rothberg, B.S., Madesh, M., and Gill, D.L. (2012). STIM proteins: dynamic calcium signal transducers. Nat. Rev. Mol. Cell Biol. 13, 549-565.

Söderberg, J.A.E., Carlsson, M.A., and Nässel, D.R. (2012). Insulin-producing cells in the Drosophila brain also express satiety-inducing cholecystokinin-like peptide, drosulfakinin. Front. Endocrinol. (Lausanne). 3, 1-13.

Song, W., Cheng, D., Hong, S., Sappe, B., Hu, Y., Wei, N., Zhu, C., O'Connor, M.B., Pissios, P., and Perrimon, N. (2017). Midgut-derived Activin regulates glucagon-like action in the fat body and glycemic Control. Cell Metab. 25, 386-399.

Soulie, J., Sheplocki, G.J., Tiany, W., and Hsuii, R.Y. (1984). Transient kinetic studies of of fatty acid synthetase. J. Biol. Chem. 259, 134-140.

Souza, S.C., Muliro, K. V., Liscum, L., Lien, P., Yamamoto, M.T., Schaffer, J.E., Dallal, G.E., Wang, X., Kraemer, F.B., Obin, M., et al. (2002). Modulation of hormone-sensitive lipase and protein kinase A-mediated lipolysis by perilipin $A$ in an adenoviral reconstituted system. $\mathrm{J}$. Biol. Chem. 277, 8267-8272.

Spandl, J., White, D.J., Peychl, J., and Thiele, C. (2009). Live cell multicolor imaging of lipid droplets with a new dye, LD540. Traffic 10, 1579-1584.

Speakman, J.R., and O'Rahilly, S. (2012). Fat: an evolving issue. Dis. Model. Mech. 5, 569573.

Speakman, J.R., Levitsky, D. a, Allison, D.B., Bray, M.S., de Castro, J.M., Clegg, D.J., Clapham, J.C., Dulloo, A.G., Gruer, L., Haw, S., et al. (2011). Set points, settling points and some alternative models: theoretical options to understand how genes and environments combine to regulate body adiposity. Dis. Model. Mech. 4, 733-745.

Spencer, I.M., and Candy, D.J. (1976). Hormonal control of diacyl glycerol mobilization from fat body of the desert locust, Schistocerca gregaria. Insect Biochem. 6, 289-296.

Spiegelman, B.M., and Flier, J.S. (2001). Obesity and the regulation of energy balance. Cell $104,531-543$

Stahl, E. (1983). A quarter century of thin-layer chromatography- an interim report. Angew. Chern. Int. Ed. Engl. 22, 507-516.

St Johnston, D. (2002). The art and design of genetic screens: Drosophila melanogaster. Nat. Rev. Genet. 3, 176-188.

Staubli, F., Jorgensen, T.J.D., Cazzamali, G., Williamson, M., Lenz, C., Sondergaard, L., Roepstorff, P., and Grimmelikhuijzen, C.J.P. (2002). Molecular identification of the insect adipokinetic hormone receptors. Proc. Natl. Acad. Sci. U. S. A. 99, 3446-3451.

Steinhauer, J., Miguel A. Gijón, Riekhof, W.R., Voelker, D.R., Murphy, R.C., and Treisman, 
J.E. (2009). Drosophila lysophospholipid acyltransferases are specifically required for germ cell development. Mol. Biol. Cell 20, 5224-5235.

Sternweis, P.C., and Smrcka, A. V (1992). Regulation of phospholipase C by G proteins. Trends Biochem. Sci. 17, 502-506.

Stevens, G.A., Singh, G.M., Lu, Y., Danaei, G., Lin, J.K., Finucane, M.M., Bahalim, A.N., McIntire, R.K., Gutierrez, H.R., Cowan, M., et al. (2012). National, regional, and global trends in adult overweight and obesity prevalences. Popul. Health Metr. 10, 22.

Stunkard, A.J., Sørensen, T.I.A., Hanis, C., Teasdale, T.W., Chakraborty, R., Schull, W.J., and Schulsinger, F. (1986). An adoption study of human obesity. N. Engl. J. Med. 314, 193198.

Sturtevant A. H. (1959). Thomas Hunt Morgan. In A Biographical Memoir of National Academy of Sciences, pp. 283-325.

Subramanian, M., Metya, S.K., Sadaf, S., Kumar, S., Schwudke, D., and Hasan, G. (2013). Altered lipid homeostasis in Drosophila InsP3 receptor mutants leads to obesity and hyperphagia. Dis. Model. Mech. 6, 734-744.

Suh, J.M., Zeve, D., McKay, R., Seo, J., Salo, Z., Li, R., Wang, M., and Graff, J.M. (2007). Adipose is a conserved dosage-sensitive antiobesity gene. Cell Metab. 6, 195-207.

Supek, F., Bošnjak, M., Škunca, N., and Šmuc, T. (2011). Revigo summarizes and visualizes long lists of gene ontology terms. PLoS One 6, e21800.

Swinburn, B., Sacks, G., and Ravussin, E. (2009). Increased food energy supply is more than sufficient to explain the US epidemic of obesity. Am. J. Clin. Nutr. 90, 1453-1456.

Swinburn, B.A., Sacks, G., Hall, K.D., McPherson, K., Finegood, D.T., Moodie, M.L., and Gortmaker, S.L. (2011). The global obesity pandemic: shaped by global drivers and local environments. Lancet 378, 804-814.

Takeuchi, K., Nakano, Y., Kato, U., Kaneda, M., Aizu, M., Awano, W., Yonemura, S., Kiyonaka, S., Mori, Y., Yamanoto, D., et al. (2009). Changes in temperature preferences and energy homeostasis in dystroglycan mutans. Science 323, 1740-1743.

Tan, J.S.Y., Seow, C.J.P., Goh, V.J., and Silver, D.L. (2014). Recent advances in understanding proteins involved in lipid droplet formation, growth and fusion. J. Genet. Genomics 41, 251-259.

Adipose tissue mTORC2 regulates ChREBP-driven de novo lipogenesis and hepatic glucose metabolism. Nat. Commun. 7, 11365.

Tansey, J.T., Sztalryd, C., Gruia-Gray, J., Roush, D.L., Zee, J. V, Gavrilova, O., Reitman, M.L., Deng, C.X., Li, C., Kimmel, A.R., et al. (2001). Perilipin ablation results in a lean mouse with aberrant adipocyte lipolysis, enhanced leptin production, and resistance to diet-induced obesity. Proc. Natl. Acad. Sci. U. S. A. 98, 6494-6499.

Teleman, A.A. (2009). Molecular mechanisms of metabolic regulation by insulin in Drosophila. Biochem. J. 425, 13-26. 
Tennessen, J.M., Barry, W.E., Cox, J., and Thummel, C.S. (2014). Methods for studying metabolism in Drosophila. Methods 68, 105-115.

Theodosiou, N.A., and Xu, T. (1998). Use of FLP/FRT system to study Drosophila development. Methods 14, 355-365.

Thomas, E.L., Fitzpatrick, J.A., Malik, S.J., Taylor-Robinson, S.D., and Bell, J.D. (2013). Whole body fat: content and distribution. Prog. Nucl. Magn. Reson. Spectrosc. 73, 56-80.

Tiefenbock, S.K., Baltzer, C., Egli, N.A., and Frei, C. (2010). The Drosophila PGC-1 homologue Spargel coordinates mitochondrial activity to insulin signalling. EMBO J. 29, 171183.

Tricoire, H., Battisti, V., Trannoy, S., Lasbleiz, C., Pret, A.M., and Monnier, V. (2009). The steroid hormone receptor EcR finely modulates Drosophila lifespan during adulthood in a sexspecific manner. Mech. Ageing Dev. 130, 547-552.

Trinh, I., and Boulianne, G.L. (2013). Modeling obesity and its associated disorders in Drosophila. Physiology 28, 117-124.

Turunen, S., and Crailsheim, K. (1996). Lipid and sugar absorpition. In Biology of Insect Midgut (London: Chapmann \& Hall, ed.).

Ugrankar, R., Liu, Y., Provaznik, J., Schmitt, S., and Lehmann, M. (2011). Lipin is a central regulator of adipose tissue development and function in Drosophila melanogaster. Mol. Cell. Biol. 31, 1646-1656.

Ugur, B., Chen, K., and Bellen, H.J. (2016). Drosophila tools and assays for the study of human diseases. Dis. Model. Mech. 9, 235-244.

Vandevijvere, S., Chow, C.C., Hall, K.D., and Swinburn, B.A. (2015). Increased food energy supply as a major driver of the obesity epidemic : a global analysis. Bull. World Heal. Organ 93, 446-456.

Vastenhouw, N.L., Brunschwig, K., Okihara, K.L., Müller, F., Tijsterman, M., and Plasterk, R.H.A. (2006). Long-term gene silencing by RNAi. Nature 442, 881-882.

Veenstra, J.A. (1994). Isolation and structure of the Drosophila corazonin gene. Biochem. Biophys. Res. Commun. 204, 292-296.

Velho, G., Petersen, K.F., Perseghin, G., Hwang, J.H., Rothman, D.L., Pueyo, M.E., Cline, G.W., Froguel, P., and Shulman, G.I. (1996). Impaired hepatic glycogen synthesis in glucokinase-deficient (MODY-2) subjects. J. Clin. Invest. 98, 1755-1761.

Venken, K.J.T., and Bellen, H.J. (2007). Transgenesis upgrades for Drosophila melanogaster. Development 134, 3571-3584.

Verleyen, P., Huybrechts, J., Baggerman, G., Van Lommel, A., De Loof, A., and Schoofs, L. (2004). SIFamide is a highly conserved neuropeptide: a comparative study in different insect species. Biochem. Biophys. Res. Commun. 320, 334-341. 
Vig, M., Peinelt, C., Beck, A., Koomoa, D.L., Rabah, D., Koblan-Huberson, M., Kraft, S., Turner, H., Fleig, A., Penner, R., et al. (2006). CRACM1 is a plasma membrane protein essential for store-operated $\mathrm{Ca}^{2+}$ entry. Science 312, 1220-1223.

Vroemen, S.F., Van Marrewijk, W.J.A., Schepers, C.C.J., and Van Der Horst, D.J. (1995). Signal transduction of adipokinetic hormones involves $\mathrm{Ca}^{2+}$ fluxes and depends on extracellular $\mathrm{Ca}^{2+}$ to potentiate cAMP-induced activation of glycogen phosphorylase. Cell Calcium 17, 459-467.

Vroemen, S.F., Van Marrewijk, W.J.A., De Meijer, J., Van Den Broek, A.T.M., and Van Der Horst, D.J. (1997). Differential induction of inositol phosphate metabolism by three adipokinetic hormones. Mol. Cell. Endocrinol. 130, 131-139.

Walker, G.E., Marzullo, P., Ricotti, R., Bona, G., and Prodam, F. (2014). The pathophysiology of abdominal adipose tissue depots in health and disease. Horm. Mol. Biol. Clin. Investig. 19, $57-74$.

Walther, T.C., and Farese, R. V (2012). Lipid droplets and cellular lipid metabolism. Annu. Rev. Biochem. 81, 687-714.

Wang, B., Moya, N., Niessen, S., Hoover, H., Mihaylova, M.M., Shaw, R.J., Yates, J.R., Fischer, W.H., Thomas, J.B., and Montminy, M. (2011). A hormone-dependent module regulating energy balance. Cell 145, 596-606.

Wang, C., Liu, Z., and Huang, X. (2012). Rab32 is important for autophagy and lipid storage in Drosophila. PLoS One 7, e32086.

Wang, H., Becuwe, M., Housden, B.E., Chitraju, C., Porras, A.J., Graham, M.M., Liu, X.N., Thiam, A.R., Savage, D.B., Agarwal, A.K., et al. (2016a). Seipin is required for converting nascent to mature lipid droplets. Elife 5, e16582.

Wang, Q.P., Lin, Y.Q., Zhang, L., Wilson, Y.A., Oyston, L.J., Cotterell, J., Qi, Y., Khuong, T.M., Bakhshi, N., Planchenault, Y., et al. (2016b). Sucralose promotes food intake through NPY and a neuronal fasting response. Cell Metab. 24, 75-90.

Wang, Z., Hayakawa, Y., and Downer, R. (1990). Factors influencing cyclic AMP and diacylglycerol levels in fat body of Locusta migratoria. Insect Biochem. 20, 325-330.

Wang, Z., Gerstein, M., and Snyder, M. (2009). RNA-Seq: a revolutionary tool for transcriptomics. Nat. Rev. Genet. 10, 57-63.

Weinkove, D., Neufeld, T.P., Twardzik, T., Waterfield, M.D., and Leevers, S.J. (1999). Regulation of imaginal disc cell size, cell number and organ size by Drosophila class I(A) phosphoinositide 3-kinase and its adaptor. Curr. Biol. 9, 1019-1029.

Wellen, K.E., and Hotamisligil, G.S. (2003). Obesity-induced inflamatory changes in adipose tissue. J. Clin. Invest. 112, 1785-1788.

Wells, J.C.K. (2012). The evolution of human adiposity and obesity: where did it all go wrong? Dis. Model. Mech. 5, 595-607.

Welte, M.A. (2015). As the fat flies: the dynamic lipid droplets of Drosophila embryos. Biochim 
Biophys. Acta 1851, 1156-1185.

Westerterp, K.R., and Speakman, J.R. (2008). Physical activity energy expenditure has not declined since the 1980s and matches energy expenditures of wild mammals. Int. J. Obes. (Lond). 32, 1256-1263.

WHO Consultation on Obesity (2000). Obesity: preventing and managing the global epidemic-report of a WHO Consultation (Geneva: World Health Organization).

Wilcox, G. (2005). Insulin and insulin resistance. Clin. Biochem. Rev. 26, 19-39.

Wilfling, F., Wang, H., Haas, J.T., Krahmer, N., Gould, T.J., Uchida, A., Cheng, J.X., Graham, M., Christiano, R., Fröhlich, F., et al. (2013). Triacylglycerol synthesis enzymes mediate lipid droplet growth by relocalizing from the ER to lipid droplets. Dev. Cell 24, 384-399.

Wilfling, F., Haas, J.T., Walther, T.C., and Jr, R.V.F. (2014). Lipid droplet biogenesis. Curr. Opin. Cell Biol. 29, 39-45.

Williams, M.J., Goergen, P., Rajendran, J., Zheleznyakova, G., Hägglund, M.G., Perland, E., Bagchi, S., Kalogeropoulou, A., Khan, Z., Fredriksson, R., et al. (2014). Obesity-linked homologues TfAP-2 and Twz establish meal frequency in Drosophila melanogaster. PLoS Genet. 10, e1004499.

Willoughby, D., and Cooper, D.M.F. (2006). $\mathrm{Ca}^{2+}$ stimulation of adenylyl cyclase generates dynamic oscillations in cyclic AMP. J. Cell Sci. 119, 828-836.

Willoughby, D., Everett, K.L., Halls, M.L., Pacheco, J., Skroblin, P., Vaca, L., Klussmann, E., and Cooper, D.M.F. (2012). Direct binding between Orai1 and AC8 mediates dynamic interplay between $\mathrm{Ca}^{2+}$ and cAMP signaling. Sci. Signal. 5, ra29.

Willyard, C. (2014). Heritability: the family roots of obesity. Nature 508, S58-60.

Woodcock, K.J., Kierdorf, K., Pouchelon, C.A., Vivancos, V., Dionne, M.S., and Geissmann, F. (2015). Macrophage-derived upd3 cytokine causes impaired glucose homeostasis and reduced lifespan in Drosophila fed a lipid-rich diet. Immunity 42, 133-144.

Woods, S.C., Lutz, T.A., Geary, N., and Langhans, W. (2006). Pancreatic signals controlling food intake; insulin, glucagon and amylin. Philos. Trans. R. Soc. Lond. B. Biol. Sci. 361, 1219-1235.

World Health Organization (2008). Waist circumference and waist-hip ratio report of a WHO expert consultation (Geneva: World Health Organization).

Wren, A.M., Small, C.J., Ward, H.L., Murphy, K.G., Dakin, C.L., Taheri, S., Kennedy, A.R., Roberts, G.H., Morgan, D.G.A., Ghatei, M.A., et al. (2000). The novel hypothalamic peptide ghrelin stimulates food intake and growth hormone secretion. Endocrinology 141, 4325-4328.

Wren, A.M., Seal, L.J., Cohen, M.A., Brynes, A.E., Frost, G.S., Murphy, K.G., Dhillo, W.S., Ghatei, M.A., and Bloom, S.R. (2001). Ghrelin enhances appetite and increases food intake in humans. J. Clin. Endocrinol. Metab. 143, 3179-3182.

Wu, Q., and Brown, M.R. (2006). Signaling and function of insulin-like peptides in insects. 
Annu. Rev. Entomol. 51, 1-24.

Wu, Q., Zhang, Y., Xu, J., and Shen, P. (2005). Regulation of hunger-driven behaviors by neural ribosomal S6 kinase in Drosophila. Proc. Natl. Acad. Sci. U. S. A. 102, 13289-13294.

Xia, Q., and Grant, S.F.A. (2013). The genetics of human obesity. Ann. N. Y. Acad. Sci. 1281, 178-190.

Yamamoto, S., Jaiswal, M., Charng, W.-L., Gambin, T., Karaca, E., Mirzaa, G., Wiszniewski, W., Sandoval, H., Haelterman, N.A., Xiong, B., et al. (2014). A Drosophila genetic resource of mutants to study mechanisms underlying human genetic diseases. Cell 159, 200-214.

Yang, J.S., Nam, H.J., Seo, M., Han, S.K., Choi, Y., Nam, H.G., Lee, S.J., and Kim, S. (2011). OASIS: online application for the survival analysis of lifespan assays performed in aging research. PLoS One 6, e23525.

Yatsenko, A.S., Marrone, A.K., Kucherenko, M.M., and Shcherbata, H.R. (2014). Measurement of metabolic rate in Drosophila using respirometry. J. Vis. Exp. 88, e51681.

Yu, Y., Huang, R., Ye, J., Zhang, V., Wu, C., Cheng, G., Jia, J., and Wang, L. (2016). Regulation of starvation-induced hyperactivity by insulin and glucagon signaling in adult Drosophila. Elife 5, e15693.

Zamore, P.D., Tuschl, T., Sharp, P. a, and Bartel, D.P. (2000). RNAi: double-stranded RNA directs the ATP-dependent cleavage of mRNA at 21 to 23 nucleotide intervals. Cell 101, 2533.

Zechner, R., Kienesberger, P.C., Haemmerle, G., Zimmermann, R., and Lass, A. (2009). Adipose triglyceride lipase and the lipolytic catabolism of cellular fat stores. J. Lipid Res. 50, 3-21.

Zhang, H., and Zhang, C. (2010). Adipose "talks" to distant organs to regulate insulin sensitivity and vascular function. Obesity (Silver Spring). 18, 2071-2076.

Zhang, H., Stallock, J.P., Ng, J.C., Reinhard, C., and Neufeld, T.P. (2000). Regulation of cellular growth by the dTOR. Genes Dev. 14, 2712-2724.

Zhang, S.L., Yu, Y., Roos, J., Kozak, J.A., Deerinck, T.J., Ellisman, M.H., Stauderman, K.A., and Cahalan, M.D. (2005). STIM1 is a $\mathrm{Ca}^{2+}$ sensor that activates CRAC channels and migrates from the $\mathrm{Ca}^{2+}$ store to the plasma membrane. Nature 437, 902-905.

Zhang, S.L., Yeromin, A. V., Zhang, X.H.-F., Yu, Y., Safrina, O., Penna, A., Roos, J., Stauderman, K.A., and Cahalan, M.D. (2006). Genome-wide RNAi screen of $\mathrm{Ca}^{2+}$ influx identifies genes that regulate $\mathrm{Ca}^{2+}$ release-activated $\mathrm{Ca}^{2+}$ channel activity. Proc. Natl. Acad. Sci. U. S. A. 103, 9357-9362.

Zhang, Y., Proenca, R., Maffei, M., Barone, M., Leopold, L., and Friedman, J.M. (1994). Positional cloning of the mouse obese gene and its human homologue. Nature 372, 425-432.

Zhao, X., Feng, D., Wang, Q., Abdulla, A., Xie, X.J., Zhou, J., Sun, Y., Yang, E.S., Liu, L.P., Vaitheesvaran, B., et al. (2012). Regulation of lipogenesis by cyclin-dependent kinase 8 mediated control of SREBP-1. J. Clin. Invest. 122, 2417-2427. 
Ziegler, R., Jasensky, R.D., and Morimoto, H. (1995). Characterization of the adipokinetic hormone receptor from the fat body of Manduca sexta. Regul. Pept. 57, 329-338.

Zimmermann, R., Strauss, J.G., Haemmerle, G., Schoiswohl, G., Birner-Gruenberger, R., Riederer, M., Lass, A., Neuberger, G., Eisenhaber, F., Hermetter, A., et al. (2004). Fat mobilization in adipose tissue is promoted by adipose triglyceride lipase. Science 306, 13831386. 


\title{
7 Promovierenden-Erklärung der Georg-August- Universität Göttingen
}

\author{
Name: Xu, Yanjun
}

Anschrift: Kellnerweg 16, 37077, Göttingen, Niedersachsen, Deutschland

Ich beabsichtige, eine Dissertation zum Thema: "Regulation of Drosophila melanogaster body fat storage by store-operated calcium entry",

an der Georg-August-Universität Göttingen anzufertigen. Dabei werde ich von Herrn Prof. Dr. Ronald P. Kühnlein betreut.

Ich gebe folgende Erklärung ab:

1. Die Gelegenheit zum vorliegenden Promotionsvorhaben ist mir nicht kommerziell vermittelt worden. Insbesondere habe ich keine Organisation eingeschaltet, die gegen Entgelt Betreuerinnen und Betreuer für die Anfertigung von Dissertationen sucht oder die mir obliegenden Pflichten hinsichtlich der Prüfungsleistungen für mich ganz oder teilweise erledigt.

2. Hilfe Dritter wurde bis jetzt und wird auch künftig nur in wissenschaftlich vertretbarem und prüfungsrechtlich zulässigem Ausmaß in Anspruch genommen. Insbesondere werden alle Teile der Dissertation selbst angefertigt; unzulässige fremde Hilfe habe ich dazu weder unentgeltlich noch entgeltlich entgegengenommen und werde dies auch zukünftig so halten.

3. Die Ordnung zur Sicherung der guten wissenschaftlichen Praxis an der Universität Göttingen wird von mir beachtet.

4. Eine entsprechende Promotion wurde an keiner anderen Hochschule im In- oder Ausland beantragt; die eingereichte Dissertation oder Teile von ihr wurden nicht für ein anderes Promotionsvorhaben verwendet.

Mir ist bekannt, dass unrichtige Angaben die Zulassung zur Promotion ausschließen bzw. später zum Verfahrensabbruch oder zur Rücknahme des erlangten Grades führen.

, den

(Ort)

(Unterschrift) 


\section{Curriculum Vitae}

\section{Personal Information}

Name: $\quad \mathrm{Xu}$, Yanjun

Birth date and place: 16. 06. 1987 in Kaifeng, Henan, China

Nationality:

Chinese

Address:

Kellnerweg 16, Göttingen, Germany

Email:

yanjun.xu@mpibpc.mpg.de or xuyjlife@Gmail.com

\section{Education:}

2013-present $\quad \mathrm{PhD}$ student at PhD program: Genes and Development

Göttingen Graduate School for Neurosciences, Biophysics, and Molecular Biosciences (GGNB), Göttingen, Germany

Supervisor: Ronald Kühnlein

2010-2013 Master of Science (MS) in Biophysics, Huazhong University of

Science and Technology, Wuhan, China

Supervisor: Zhengxing $\mathrm{Wu}$

2006-2010 Bachelor of Science (BS) in Biology, Henan Agricultural University, Zhengzhou, China

2000-2006 Tian Jiabing Experimental High School, Kaifeng, China

1998-2000 Mashijie Primary School, Kaifeng, China

1994-1998 Gumen Primary School, Kaifeng, China

\section{Research training experience:}

2013- present PhD student at the Research Group of Molecular Physiology (Prof. Dr. Ronald Kühnlein), and Department of Molecular Developmental Biology (Prof. Dr. Herbert Jäckle), Max Planck Institute for Biophysical Chemistry, Göttingen, Germany

2010-2013 Master student in the lab of Prof. Dr. Zhengxing Wu, College of Life Science \& Technology, Huazhong University of Science \& Technoglogy, Wuhan, China 


\section{Professional skills:}

\section{Language skills}

Chinese: native-speaker, English: IELTS (7.5/9), German: B1

\section{Data processing, visualization and bioinformatics:}

RNAseq analysis, data quality analysis (Clustering analysis, Principal Component Analysis) and gene differential expression analysis, Gene Ontology (GO) enrichment analysis, Pathway analysis. Network analysis with Python.

Cell size and lipid droplets (LD) size quantification by image $\mathrm{J}$.

Primary data processing and figure with Excel,

Statistical analysis and figure with R and SPSS,

Figure, poster with Adobe Illustrator

\section{Experiments:}

Molecular cloning, RT-qPCR (SYBR, TaqMan), Western blotting, ELISA, Chromatin immunoprecipitation (ChIP), Lipid droplets staining, Immunostaining and confocal imaging, haematoxylin \& eosin (H\&E) staining and paraffin embedded tissue section, Fly body fat/protein/glycogen/glucose/ATP content measurements (enzymatic assays), Lipid fraction by Thin layer chromatography (TLC) or Column solid phase extraction (SPE), ${ }^{14} \mathrm{C}$ pulse chase assay, neutral lipid analysis (non polar lipids converted fatty acid methyl esters (FAME) by GC-MS),

C. elegans genetics and handling, Drosophila genetics and handling, Adult fly organ dissection (fat body tissue, corpora cardiaca, brain), Food intake assay (CAFE), Metabolic rate assay, Locomotor assay, Climbing ability assay, Starvation resistance assay, Life span assay.

\section{Supervision of students and teaching experience:}

Bachelor thesis:

Annika Franziska Borcherding: A genetic screen identifies candidate genes which lead to programmed obesity by transient Knock Down in Drosophila. Georg-August University Göttingen.

Erasmus Master student internship:

Pilar Mata Tutor: Drosophila molecular genetics. Max Planck Institute for Biophysical Chemistry, Göttingen

Collaboration with student assistants: 
Mike Piezarek, Vanessa Kernke, Jana Laura Heidemann (Max Planck Institute for Biophysical Chemistry, Göttingen)

Teaching assistant for Medical biochemistry practical course: Hormone I (Glucagon) and II (Steroids), University Medical Center Göttingen.

\section{Peer-Reviewed Publications:}

First co-author (equal contribution, indicated by *) publication:

1. Baumbach, J., Xu, Y., Hehlert, P., and Kühnlein, R.P. (2014b). Gaq, Gy1 and Plc21C control Drosophila body fat storage. J. Genet. Genomics 41, 283-292. doi:10.1016/j.jgg.2014.03.005.

2. Huang $W^{*} M^{*}$ Li $Z Y^{*}, \mathbf{X u}, \mathbf{Y}^{\star}$ et al., (2014). PKG and NHR-49 signalling coordinately regulate short-term fasting-induced lysosomal lipid accumulation in $C$. elegans. Biochem J. 461, 509-520. doi: 10.1042/BJ20140191.

Co-author Publications:

1. Gáliková, M., Klepsatel, P., Xu, Y., and Kühnlein, R.P. (2017). The obesity-related adipokinetic hormone controls feeding and expression of neuropeptide regulators of Drosophila metabolism. Eur. J. Lipid Sci. Technol. 119. 1600138. doi:10.1002/ejlt.201600138.

2. Klepsatel, P., Gáliková, M., Xu, Y., and Kühnlein, R.P. (2016). Thermal stress depletes energy reserves in Drosophila. Sci. Rep. 6, 33667. doi: 10.1038/srep33667.

3. Gáliková, M., Diesner, M., Klepsatel, P., Hehlert, P., Xu, Y., Bickmeyer, I., Predel, R., and Kühnlein, R.P. (2015). Energy homeostasis control in Drosophila adipokinetic hormone mutants. Genetics 201, 665-683. doi: 10.1534/genetics.115.178897.

\section{Awards}

2017 GGNB Bridging Fund-Postdoktorandenstipendium

2013 Max Planck PhD fellowship

2010-13 Graduate Studies Scholarship,

Huazhong University of Science \& Technology, China

2009 National Encouragement Scholarship, China

2007 National Scholarship, China 